
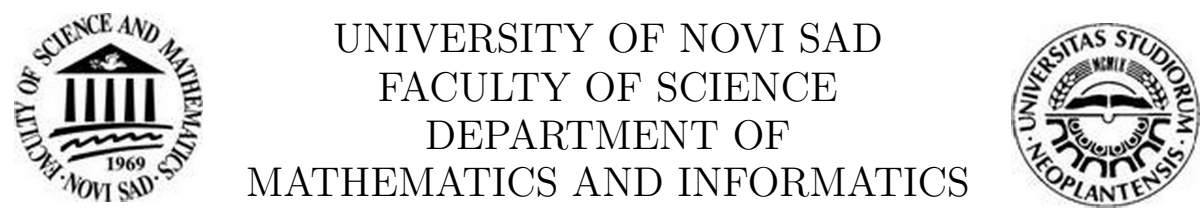

Boris Šobot, M.Sc.

\title{
GAMES ON BOOLEAN ALGEBRAS
}

-Ph.D. thesis-

Supervisor: Professor Miloš Kurilić, Ph.D.

Novi Sad, 2009. 



\section{Contents}

1 Forcing $\quad 7$

1.1 Notation and preliminaries . . . . . . . . . . . 7

1.2 Partial orders and complete Boolean algebras . . . . . . . . 10

1.3 Basics of forcing . . . . . . . . . . . . . . . . . . 14

1.4 Chain conditions and distributivity . . . . . . . . . 28

1.5 Proper, Sacks, Laver and ${ }^{\omega} \omega$-bounding notions of forcing . . . 34

1.6 Various notions of forcing . . . . . . . . . . 36

2 Cut-and-choose games on Boolean algebras 43

2.1 Infinite games. The Banach-Mazur game . . . . . . . . . . . 43

2.2 Games on Boolean algebras. The game $\mathcal{G}_{\text {inf }}(\kappa) \ldots \ldots$

2.3 The cut-and-choose games . . . . . . . . . . . . . . 51

2.4 The game $\mathcal{G}_{\text {dist }}(\kappa, \lambda, \mu) \ldots \ldots \ldots \ldots \ldots$

2.5 The distributivity game on partial orders . . . . . . . . . 64

2.6 The games $\mathcal{G}_{\mathrm{sp}}, \mathcal{G}_{\mathrm{b}}$ and $\mathcal{G}_{\mathrm{lp}} \ldots \ldots \ldots \ldots$

3 A power collapsing game $\quad \mathbf{7 9}$

3.1 The game $\mathcal{G}_{\mathrm{ls}}(\kappa)$ from White's point of view . . . . . . . . 80

3.2 The game $\mathcal{G}_{\mathrm{ls}}(\kappa)$ from Black's point of view . . . . . . . . 87

3.3 Some additional results . . . . . . . . . . . . . . . 90

3.4 The indeterminacy . . . . . . . . . . . . . . . . . 93 



\section{Preface}

The theory of infinite games has become, in the last few decades, a very important topic in set theory. The main object of investigating games is to obtain more information on various objects in set theoretical universe, by finding game-theoretic equivalents of statements about these objects.

An infinite game played on Boolean algebras first appeared in [15]. Since then, many distinguished scientists in this area participated in constructing and discovering interesting properties of such games.

For most set theorists nowadays, Boolean algebras are firmly connected to forcing. Many properties of Boolean algebras can be expressed in terms of their forcing properties. This is why a major part of Chapter 1 of this thesis is devoted to this method. After mentioning the prerequisites, we introduce some definitions and turn to the foundation of forcing. Both approaches, via Boolean algebras and via partial orders are described, and then they are compared. Some important properties of Boolean algebras are introduced, such as chain conditions and distributivity laws. The end of the chapter contains definitions of all notions of forcing that will be used throughout the thesis.

The description of games on Boolean algebras begins in Chapter 2. At the beginning some general notation is established, and the notions of a play, a partial play and a winning strategy are introduced. The first game considered is the Banach-Mazur game. It is of great historical importance, but it also served as an inspiration for Jech's descending chain game, the one that we describe next. From that point on, we turn our attention to socalled "cut-and-choose" games, the main subject of the thesis. Since some properties hold for all such games, we prove them first. Then we describe another Jech's game, characterizing distributivity laws, that was thoroughly investigated. We also define its equivalent on partial orders. Finally, three more games, introduced by Kada, are mentioned.

The last chapter is devoted to the game $\mathcal{G}_{\mathrm{ls}}$, characterizing a collapsing property of Boolean algebras. A special (countable) case of this game was first defined by Professor Miloš Kurilić and myself in [25], and the general game was introduced in [26]. The game is investigated from the point of view of each of the players, and a Boolean algebra is defined on which neither of them has a winning strategy. The results in this chapter, as well as the results in sections 2.3 and 2.5, present our contribution to the theory.

While writing this material, I tried to make it as self-contained as possible, providing a proof or a reference for every result (for classical results references are usually made to textbooks [19] and [24], rather than to pa- 
pers where they were proved). In particular, I included proofs that are, in my opinion, useful for understanding the material, and proofs that are commonly skipped in the textbooks. On the other hand, long and technical proofs are usually not included, with the exception of Chapter 3 , where all the results are proved in detail.

I wish to express my gratitude to my supervisor, Professor Miloš Kurilić, who initiated our work in this area, led the research, and finally made many suggestions that helped me to improve the thesis. I also wish to thank Professor Milan Grulović who is responsible for my interest in set theory. Finally, my family is a motive and an inspiration for everything I do, and so it was during the writing of this thesis.

Novi Sad, 21. 4. 2009.

Boris Šobot 


\section{Chapter 1}

\section{Forcing}

In this chapter we give some definitions and facts needed for understanding the main part of the thesis, as well as some examples that will be used later.

\subsection{Notation and preliminaries}

Throughout this thesis it will be understood that the reader has the knowledge of ZFC axioms and their basic corollaries, such as recursive definitions, inductive proofs, the cumulative hierarchy $V_{\alpha}(\alpha \in$ Ord), the constructible universe $L$, models of set theory, some cardinal arithmetic etc. All this can be found in textbooks, for example [19] or [24].

Here is some notation we will need. We will denote cardinals by $\kappa, \lambda, \mu, \ldots$ and ordinals in general by $\alpha, \beta, \gamma, \ldots$ or $\xi, \eta, \zeta \ldots$ The sequences (including ordered pairs) will be written in "angle" parenthesis $\langle$ and $\rangle$, with occasional abuse of notation; for example $\left\langle\langle a, b\rangle,\left\langle\left\langle x_{n}, y_{n}\right\rangle: n \in \omega\right\rangle\right\rangle$ is a sequence of ordered pairs, with $\langle a, b\rangle$ as the first, and $\left\langle x_{n}, y_{n}\right\rangle$ as the $n+2$-nd element $(n \in \omega)$.

We will use the following notation:

Ord - the class of all ordinals;

Lim - the class of all limit ordinals;

Card - the class of all cardinals;

Reg - the class of all regular cardinals;

${ }^{A} B$ - the set of all functions mapping $A$ to $B$;

${ }^{<\alpha} B=\bigcup_{\beta<\alpha}{ }^{\beta} B$; 
$P(A)$ - the power set of $A$;

$[A]^{\kappa}$ - the set of all subsets of $A$ of cardinality $\kappa$;

$[A]^{<\kappa}$ - the set of all subsets of $A$ of cardinality less than $\kappa$.

To denote the inclusion between two sets, we use $\subseteq$ and $\supseteq$. If we want to emphasize that the inclusion is strict, we use $\subset$ and $\supset$. If we consider a sequence $x$ of elements of a set $A$ and an element $a \in A$, with $x^{\wedge} a$ we denote the sequence obtained by adding $a$ to the end of $x$. If $f: A \rightarrow B$ is a function, for $X \subseteq A$ and $Y \subseteq B$ we also denote $f[X]=\{b \in B: \exists x \in X f(x)=b\}$ and $f^{-1}[Y]=\{a \in A: f(a) \in Y\}$. The domain $A$ of $f$ will be denoted by $\operatorname{dom} f$, and the $\operatorname{rank} f[A]$ of $f$, by $\operatorname{ran} f$.

Definition 1.1 Let $\alpha$ be a limit ordinal. An increasing sequence $\left\langle\alpha_{\xi}: \xi<\right.$ $\beta\rangle$ of ordinals less than $\alpha$ is cofinal in $\alpha$ if for every $\gamma<\alpha$ there is $\xi<\beta$ such that $\gamma \leq \alpha_{\xi}$. The cofinality of $\alpha$, denoted by $\operatorname{cf}(\alpha)$, is $\min \{\beta \in \operatorname{Ord}$ : there is a sequence of length $\beta$ cofinal in $\alpha$ \}.

Lemma $1.2([19]$, Lemma 3.8) $\operatorname{cf}(\alpha)$ is a regular cardinal for every $\alpha \in \operatorname{Lim}$.

Definition 1.3 If $\kappa$ is an uncountable cardinal, a subset $C \subseteq \kappa$ is closed unbounded (or just "club") if:

(i) it is closed, i.e. $\sup D \in C$ for every nonempty $D \subseteq C$;

(ii) it is unbounded, i.e. for every $\alpha<\kappa$ there is $\beta \geq \alpha$ such that $\beta \in C$.

A set $S \subseteq \kappa$ is stationary if it intersects every club in $\kappa$.

Lemma 1.4 ([24], Lemma II 6.13) If $\kappa$ is an uncountable regular cardinal and $f: \kappa \rightarrow \kappa$ is a function, then $\{\alpha<\kappa: f[\alpha] \subseteq \alpha\}$ is a club in $\kappa$.

Lemma 1.5 ([19], Theorem 8.3) The intersection of less than $\kappa$ club subsets of $\kappa$ is a club in $\kappa$.

Definition 1.3 can be generalized:

Definition 1.6 If $\kappa$ is an uncountable cardinal, $C \subseteq[\kappa]^{\omega}$ is closed unbounded if:

(i) it is closed, i.e. $\bigcup_{n \in \omega} D_{n} \in C$ for every countable increasing chain $D_{0} \subseteq D_{1} \subseteq D_{2} \subseteq \ldots$ of elements of $C$; 
(ii) it is unbounded, i.e. for every $X \in[\kappa]^{\omega}$ there is $Y \in C$ such that $X \subseteq Y$.

A set $S \subseteq[\kappa]^{\omega}$ is stationary if it intersects every club in $[\kappa]^{\omega}$.

We will now state some additional axioms. If $\kappa$ is a regular cardinal and $S$ is a stationary subset of $\kappa$, by $\nabla_{\kappa}(S)$ we will denote the following statement:

$\nabla_{\kappa}(S)$ : There are sets $A_{\gamma} \subseteq \gamma$ for $\gamma \in S$ such that for each $A \subseteq \kappa$ the set $\left\{\gamma \in S: A \cap \gamma=A_{\gamma}\right\}$ is a stationary subset of $\kappa$.

The special case $\nabla_{\kappa^{+}}(E(\kappa))$, where $E(\kappa)=\left\{\alpha<\kappa^{+}: \operatorname{cf}(\alpha)=\kappa\right\}$, will be the most important for us. In another special case, $S=\kappa$, we will write only $\nabla_{\kappa}$ instead of $\nabla_{\kappa}(\kappa)$. For $\kappa=\omega_{1}$ we get the well-known Jensen's ${ }^{1}$ diamond principle:

$\diamond:$ There are sets $A_{\gamma} \subseteq \gamma$ for $\gamma \in \omega_{1}$ such that for each $A \subseteq \omega_{1}$ the set $\left\{\gamma \in \omega_{1}: A \cap \gamma=A_{\gamma}\right\}$ is a stationary subset of $\omega_{1}$.

Lemma 1.7 (a) $V=L \vdash \forall \kappa \in \operatorname{Reg} \forall S\left(S\right.$ is stationary in $\left.\kappa \Rightarrow \diamond_{\kappa}(S)\right)$.

(b) If $S_{1}$ and $S_{2}$ are stationary in $\kappa$ and $S_{1} \subseteq S_{2}$, then $\diamond_{\kappa}\left(S_{1}\right) \Rightarrow \nabla_{\kappa}\left(S_{2}\right)$.

(c) For each regular cardinal $\kappa, \diamond_{\kappa^{+}} \Rightarrow 2^{\kappa}=\kappa^{+}$.

Proof. (a) This is a generalization of Jensen' Theorem $V=L \vdash \diamond$ (see [19], Theorem 13.21).

(b) Follows from the fact that a subset of $\kappa$ containing a stationary set is stationary.

(c) Assume $\nabla_{\kappa^{+}}$, let $\left\langle A_{\gamma}: \gamma \in \kappa^{+}\right\rangle$be the sequence thus obtained, and let $A \subseteq \kappa$. The sets $S_{A}=\left\{\gamma<\kappa^{+}: A=A_{\gamma}\right\}$ and $S_{A}^{\prime}=\left\{\gamma<\kappa^{+}: A \cap \gamma=A_{\gamma}\right\}$ may differ only in elements $\gamma<\sup A$, so since $S_{A}^{\prime}$ is stationary in $\kappa^{+}, S_{A}$ must be stationary too. But then $\left|S_{A}\right|=\kappa^{+}$. Hence $|P(\kappa)|>\kappa^{+}$would imply $\left|\bigcup_{A \in P(\kappa)} S_{A}\right|>\kappa^{+}$(because the sets $S_{A}$ are pairwise disjoint), which is a contradiction since $\bigcup_{A \in P(\kappa)} S_{A} \subseteq \kappa^{+}$.

We remark that conditions $\diamond_{\kappa^{+}}(E(\kappa))$ and $\diamond_{\kappa^{+}}$are equivalent for $\kappa=\omega$.

\footnotetext{
${ }^{1}$ Ronald B. Jensen (b. 1936), American mathematician
} 
Finally, " $0^{\sharp}$ exists" is another axiom we will need, introduced by Solovay $^{2}$. It claims the existence of the set

$$
0^{\sharp}=\left\{\varphi\left(x_{1}, \ldots, x_{n}\right): L_{\aleph_{\omega}}=\varphi\left(\aleph_{1}, \ldots, \aleph_{n}\right)\right\} .
$$

Part of its strength lies in providing a truth definition for the constructible universe (it is known that a truth definition in general does not exist by Tarski's Theorem, see [19], Theorem 12.7). To fully understand $0^{\sharp}$ it is necessary to get acquainted with Silver ${ }^{3}$ indiscernibles; see [19], page 311 for more information and some important corollaries of the nonexistence of $0^{\sharp}$. However, the nonexistence of $0^{\sharp}$ is equivalent to a property that is quite simple to formulate; we will need only one implication of this equivalence.

Theorem 1.8 (Jensen's Covering Theorem) ([19], Theorem 18.30) If $0 \sharp$ does not exist, then for every uncountable set $X$ of ordinals there is a constructible $Y \supseteq X$ of the same cardinality.

\subsection{Partial orders and complete Boolean algebras}

Partial orders and Boolean algebras will be denoted by blackboard-bold letters, $\mathbb{P}$ for $\langle P, \leq\rangle$ and $\mathbb{B}$ for $\left\langle B, \wedge, \vee,^{\prime}, 0,1\right\rangle$ respectively. When only their domains are considered however, we will use ordinary $P$ and $B$. We also denote $B^{+}=B \backslash\{0\}$, and whenever we consider a Boolean algebra as a partial order, it will be understood that we consider $\left\langle B^{+}, \leq\right\rangle$.

Definition 1.9 Let $\mathbb{P}$ be a partial order, $X \subseteq P, Y \subseteq P$ and $p, q \in P$.

- $(p, q)_{\mathbb{P}}=\{r \in P: p<r<q\}$ and $[p, q]_{\mathbb{P}}=\{r \in P: p \leq r \leq q\}$.

- $X$ is dense in $\mathbb{P}$ if $\forall r \in P \exists s \in X s \leq r$.

- $X$ is open dense in $\mathbb{P}$ if it is dense and $\forall r \in X \forall s \in P(s \leq r \Rightarrow s \in X)$.

- $X \downarrow=\{r \in P: \exists x \in X r<x\}$; in particular $p \downarrow=\{r \in P: r<p\}$.

- $X$ is dense below $p$ if $\forall r \leq p \exists s \in X s \leq r$.

- $X$ is empty below $p$ if $\neg \exists r \in X r \leq p$.

- $p$ and $q$ are incompatible (we write $p \perp q)$ if $\neg \exists r \in P(r \leq p \wedge r \leq q$ ); otherwise they are compatible.

\footnotetext{
${ }^{2}$ Robert M. Solovay (b. 1938), American mathematician

${ }^{3}$ Jack H. Silver, contemporary American mathematician
} 
- $p$ is incompatible with $X$ (again, we write $p \perp X$ ) if $p$ is incompatible with every element of $X$.

- $X$ and $Y$ are incompatible if every element of $X$ is incompatible with $Y$.

For a Boolean algebra $\mathbb{B}$ we define

$$
\pi(\mathbb{B})=\min \{|D|: D \text { is dense in } \mathbb{B}\} .
$$

Definition 1.10 A partial order $\mathbb{P}$ is separative if

$$
\forall p, q \in P(\neg p \leq q \Rightarrow \exists r \leq p \quad r \perp q) .
$$

Definition 1.11 A Boolean algebra $\mathbb{B}$ is complete if every subset $X \subseteq B$ has supremum and infimum.

To avoid robust conditions in theorems, we will often assume that Boolean algebras are complete, although sometimes a weaker condition may suffice.

Theorem 1.12 ([19], Theorem 14.10) For every separative partial order $\mathbb{P}$ there is, unique up to isomorphism, a complete Boolean algebra $\mathbb{B}$ and a one-to-one mapping $e: P \rightarrow B^{+}$such that

1) $p_{1} \leq p_{2} \Leftrightarrow e\left(p_{1}\right) \leq e\left(p_{2}\right)$;

2) $e[P]$ is dense in $\mathbb{B}$.

Definition 1.13 The unique complete Boolean algebra mentioned in Theorem 1.12 is called the completion of $\mathbb{P}$ and denoted r.o.(P).

The map $e$ from Theorem 1.12 is called the canonical embedding of $\mathbb{P}$ in $\mathbb{B}$. Note that, if $p_{1}, p_{2} \in P$, then $p_{1} \perp p_{2}$ iff $e\left(p_{1}\right) \perp e\left(p_{2}\right)$. In order to simplify writing, we will often identify elements of $P$ with their images via $e$. The notation r.o. $(\mathbb{P})$ originates from the notion of regular open sets. More details on this and other facts concerning the completion of a separative partial order can be found in [30].

Lemma 1.14 For every partial order $\mathbb{P}$ there is a separative partial order $\mathbb{Q}$ and a mapping $h: P \rightarrow Q$ such that

1) $p_{1} \leq p_{2} \Rightarrow h\left(p_{1}\right) \leq h\left(p_{2}\right)$;

2) $p_{1} \perp p_{2} \Leftrightarrow h\left(p_{1}\right) \perp h\left(p_{2}\right)$. 
Proof. Let $\langle P, \leq\rangle$ be an arbitrary partial order. Obviously, the relation $\sim$ on $P$ defined by

$$
p \sim q \text { iff } \forall r \in P(r \text { is compatible with } p \Leftrightarrow r \text { is compatible with } q)
$$

is an equivalence relation. Thus, if we define a relation $\preceq$ on the quotient set $P / \sim$ as follows:

$$
[p] \preceq[q] \quad \text { iff } \quad \forall r \in P(r \leq p \Rightarrow r \text { is compatible with } q),
$$

then $\langle P / \sim, \preceq\rangle$ is clearly a separative partial order. The function $h: P \rightarrow$ $P / \sim$ defined by $h(p)=[p]$ clearly satisfies 1$)$ and 2$)$.

The partial order $\mathbb{Q}$ from the lemma above is called the separative quotient of $\mathbb{P}$. When proving theorems about forcing we will restrict our attention to separative partial orders; Lemma 1.43 allows us to do this. However, combining Lemma 1.14 with Theorem 1.12 we can also define the completion of an arbitrary partial order:

Theorem 1.15 ([19], Corollary 14.12) For every partial order $\mathbb{P}$ there is, unique up to isomorphism, a complete Boolean algebra $\mathbb{B}$ and a mapping $e: P \rightarrow B^{+}$such that

1) $p_{1} \leq p_{2} \Rightarrow e\left(p_{1}\right) \leq e\left(p_{2}\right)$

2) $p \perp q \Leftrightarrow e(p) \perp e(q)$;

3) $e[P]$ is dense in $\mathbb{B}$.

Definition 1.16 A collection $\left\{b_{\alpha}: \alpha<\kappa\right\}$ of elements of a partial order $\mathbb{P}$ is an antichain if $b_{\alpha} \perp b_{\beta}$ for $\alpha<\beta<\kappa$. An antichain $A$ is maximal if there is no $p \in P$ such that $A \cup\{p\}$ is also an antichain.

Definition 1.17 Let $\kappa$ be an infinite cardinal. A partial order $\mathbb{P}$ satisfies the $\kappa$-chain condition (shorter: the $\kappa$-c.c.) if there are no antichains of cardinality $\kappa$ in $\mathbb{P}$. In particular, if there are no uncountable antichains in $\mathbb{P}$, we say that $\mathbb{P}$ satisfies the countable chain condition (c.c.c.). We also denote

$$
\text { c.c. }(\mathbb{P})=\min \{\kappa \in \text { Card }: \mathbb{P} \text { satisfies the } \kappa-\text { c.c. }\}
$$

Lemma 1.18 If $\mathbb{P}$ is a partial order and $\mathbb{B}=$ r.o. $(\mathbb{P})$, then c.c. $(\mathbb{P})=$ c.c. $(\mathbb{B})$.

Proof. Every antichain in $\mathbb{P}$ is also an antichain in $\mathbb{B}$, so c.c. $(\mathbb{P}) \leq$ c.c. $(\mathbb{B})$. Let us prove that the other inequality holds. Let $A$ be an antichain in $\mathbb{B}$. Then there is an antichain in $\mathbb{P}$ of the same cardinality: below every $a \in A$ we find $p_{a} \in P$ such that $p_{a} \leq a$; then $\left\{p_{a}: a \in A\right\}$ is the antichain we wanted. 
Lemma 1.19 ([19], Theorem 7.15) For every partial order $\mathbb{P}$ c.c. $(\mathbb{P})$ is a regular uncountable cardinal.

Lemma 1.20 If $\mathbb{B}=$ r.o. $(\mathbb{P})$ and c.c. $(\mathbb{P})=\kappa$, then $|B| \leq|P|^{<\kappa}$.

Proof. We define $f: B \rightarrow[P]^{<\kappa}$ in the following way: for every $b \in B$ we choose a maximal antichain $f(b)$ of elements of $P$ below $b$. This mapping is obviously one-to-one, because if $b_{1} \neq b_{2}$ then (in $\left.\mathbb{B}\right) \vee f\left(b_{1}\right)=b_{1} \neq b_{2}=$ $\bigvee f\left(b_{2}\right)$, hence $f\left(b_{1}\right) \neq f\left(b_{2}\right)$.

Definition $1.21 G$ is a filter on a partial order $\mathbb{P}$ if

(i) $\emptyset \neq G \subseteq P$;

(ii) if $p \in G, q \in P$ and $p \leq q$, then $q \in G$;

(iii) if $p, q \in G$, then there is $r \in G$ such that $r \leq p$ and $r \leq q$.

Definition 1.22 $G$ is an ultrafilter on a Boolean algebra $\mathbb{B}$ if

(i) $\emptyset \neq G \subseteq B$;

(ii) if $b \in G, a \in B$ and $b \leq a$, then $a \in G$;

(iii) if $a, b \in G$, then $a \wedge b \in G$;

(iv) for each $b \in B$ either $b \in G$ or $b^{\prime} \in G$.

If $\kappa$ is an infinite cardinal, an ultrafilter on the Boolean algebra $\langle P(\kappa), \cap$, $\left.\cup,^{\prime}, \emptyset, \kappa\right\rangle$ is also called an ultrafilter on $\kappa$.

Definition 1.23 If $\kappa$ and $\lambda$ are infinite cardinals, an ultrafilter $G$ on $\kappa$ is $\lambda$-complete if, whenever $\mu<\lambda$ and $X_{\alpha} \in G$ for $\alpha<\mu, \bigcap_{\alpha<\mu} X_{\alpha} \in G$ as well. An uncountable cardinal $\kappa$ is measurable if there is a $\kappa$-complete ultrafilter on $\kappa$.

Definition 1.24 Let $\left\langle X_{\alpha}: \alpha<\kappa\right\rangle$ be a sequence of subsets of an infinite cardinal $\kappa$. The diagonal intersection of sets $X_{\alpha}$ is the set $\triangle_{\alpha<\kappa} X_{\alpha}=\{\xi<$ $\left.\kappa: \xi \in \bigcup_{\alpha<\xi} X_{\alpha}\right\}$. A $\kappa$-complete ultrafilter $G$ on $\kappa$ is normal if it is closed under diagonal intersections, i.e. whenever $X_{\alpha} \in G$ for $\alpha<\kappa, \triangle_{\alpha<\kappa} X_{\alpha} \in G$ as well. 
More on measurable cardinals and normal ultrafilters can be found in [19], page 127.

Another additional axiom, very useful in consistency proofs is the Mar$\operatorname{tin}^{4}$ Axiom. It states that for all $\kappa<\mathfrak{c} \mathrm{MA}_{\kappa}$ holds, where

$\mathrm{MA}_{\kappa}:$ If $\mathbb{P}$ is a c.c.c. partial order and $\mathcal{D}$ a collection of at most $\kappa$ sets dense in $\mathbb{P}$, then there is a filter on $\mathbb{P}$ that intersects all elements of $\mathcal{D}$.

Lemma 1.25 ([24], Theorem II 2.18) $\mathrm{MA}_{\kappa}$ implies $2^{\kappa}=\mathfrak{c}$.

Many facts concerning MA can be found in [27].

\subsection{Basics of forcing}

The method of forcing is widely used in consistency proofs. It was introduced by Cohen ${ }^{5}$ in [6] and [7]. Starting with a model $\mathcal{M}$ of $\mathrm{ZF}(\mathrm{C})$, and a partial order $\mathbb{P}$ in $\mathcal{M}$ (or its completion $\mathbb{B}=$ r.o. $(\mathbb{P})$ ), it produces another model of $\mathrm{ZF}(\mathrm{C})$. It is customary to work with the universe $V$ instead of a model $\mathcal{M}$ in general. $\mathbb{P}$ is also called a notion of forcing, and elements of $P$ are called forcing conditions.

Definition 1.26 A filter $G$ on $\mathbb{P}$ is $\mathbb{P}$-generic over $V$ if for every set $D \in V$ dense in $\mathbb{P}, G \cap D \neq \emptyset$.

Definition 1.27 An ultrafilter $G$ on $\mathbb{B}$ is $\mathbb{B}$-generic over $V$ if, for all $X \subseteq G$ such that $X \in V, \bigcap X \in G$ holds.

The following lemma is easy to prove.

Lemma 1.28 Let $\mathbb{P}$ be a partial order, $\mathbb{B}=$ r.o.( $(\mathbb{P})$ and let $e: P \rightarrow B^{+}$be the canonical embedding.

(a) If $F$ is a $\mathbb{P}$-generic filter, then

$$
F^{\prime}=\{b \in B: \exists p \in F e(p) \leq b\}
$$

is a $\mathbb{B}$-generic ultrafilter.

\footnotetext{
${ }^{4}$ Donald A. Martin (b. 1940), American mathematician

${ }^{5}$ Paul J. Cohen (1934-2007), American mathematician
} 
(b) If $G$ is a $\mathbb{B}$-generic ultrafilter, then

$$
G^{*}=\{p \in P: e(p) \in G\}
$$

is a $\mathbb{P}$-generic filter.

It is clear that $\left(F^{\prime}\right)^{*}=F$ and $\left(G^{*}\right)^{\prime}=G$, i.e. the operations of getting a $\mathbb{B}$-generic ultrafilter from a $\mathbb{P}$-generic filter and getting a $\mathbb{P}$-generic filter from a $\mathbb{B}$-generic ultrafilter are mutually inverse.

Now we define a Boolean-valued model $V^{\mathbb{B}}$ in $V$. Informally, a Booleanvalued model differs from "usual" models in the fact that formulas need not only be true (1) or false (0), but they take values from a Boolean algebra $\mathbb{B}$ (more on Boolean-valued models can be found in [5]).

Definition 1.29 Let $\mathbb{B}$ be a complete Boolean algebra. A Boolean-valued model is $\langle A, I, E\rangle$, where $A$ is a set called the Boolean universe, and $I$ : $A^{2} \rightarrow B$ and $E: A^{2} \rightarrow B$ are functions such that, for $\sigma, \tau, \mu, \nu \in A$ (writing $\|\sigma=\tau\|$ and $\|\sigma \in \tau\|$ instead of $I(\sigma, \tau)$ and $E(\sigma, \tau)$ respectively)

(i) $\|\sigma=\sigma\|=1$,

(ii) $\|\sigma=\tau\|=\|\tau=\sigma\|$,

(iii) $\|\sigma=\tau\| \wedge\|\tau=\mu\| \leq\|\sigma=\mu\|$,

(iv) $\|\sigma \in \tau\| \wedge\|\mu=\sigma\| \wedge\|\nu=\tau\| \leq\|\mu \in \nu\|$.

The intuition behind this is that $\|\sigma=\tau\|$ is the measure of equality, and $\|\sigma \in \tau\|$ the measure of membership between two elements of $A$.

Let us define the sets $V_{\alpha}^{\mathbb{B}}$ by recursion on $\alpha \in$ Ord:

$$
\begin{aligned}
& V_{0}^{\mathbb{B}}=\emptyset \\
& V_{\alpha+1}^{\mathbb{B}}=\left\{f: f \text { is a function } \wedge \operatorname{dom} f \subseteq V_{\alpha}^{\mathbb{B}} \wedge \operatorname{ran} f \subseteq B\right\} \\
& V_{\alpha}^{\mathbb{B}}=\bigcup_{\beta<\alpha} V_{\beta}^{\mathbb{B}} \text { if } \alpha \text { is a limit ordinal. }
\end{aligned}
$$

Finally, our Boolean universe will be the class

$$
V^{\mathbb{B}}=\bigcup_{\alpha \in \text { Ord }} V_{\alpha}^{\mathbb{B}}
$$


For $\sigma \in V^{\mathbb{B}}$ let $\rho(\sigma)=\min \left\{\alpha \in\right.$ Ord : $\left.\sigma \in V_{\alpha+1}^{\mathbb{B}}\right\}$; this is called the rank of $\sigma$. (There should be no confusion with the usual notion of rank.) Ordering $\left(V^{\mathbb{B}}\right)^{2}$ by the relation:

$$
\left(\sigma_{1}, \tau_{1}\right) \prec\left(\sigma_{2}, \tau_{2}\right) \Leftrightarrow \rho\left(\sigma_{1}\right)<\rho\left(\sigma_{2}\right) \vee\left(\rho\left(\sigma_{1}\right)=\rho\left(\sigma_{2}\right) \wedge \rho\left(\sigma_{1}\right)<\rho\left(\sigma_{2}\right)\right)
$$

and introducing the Boolean operation $\Rightarrow$ by $x \Rightarrow y=x^{\prime} \vee y$ we can define by recursion on pairs $\langle\sigma, \tau\rangle \in\left(V^{\mathbb{B}}\right)^{2}$ :

$$
\begin{aligned}
\|\sigma \in \tau\| & =\bigvee_{\mu \in \operatorname{dom}(\tau)}(\|\sigma=\mu\| \wedge \tau(\mu)) \\
\|\sigma \subseteq \tau\| & =\bigwedge_{\mu \in \operatorname{dom}(\sigma)}(\sigma(\mu) \Rightarrow\|\mu \in \tau\|) \\
\|\sigma=\tau\| & =\|\sigma \subseteq \tau\| \wedge\|\tau \subseteq \sigma\|
\end{aligned}
$$

Having determined the values of atomic formulas, defining Boolean values of other first-order formulas is quite natural. Introducing another operation $\Leftrightarrow$ by $x \Leftrightarrow y=(x \Rightarrow y) \wedge(y \Rightarrow x)$, let us define $\|\varphi(\sigma)\|$ for $\sigma \in V^{\mathbb{B}}$ by recursion on the complexity of the formula $\varphi(x)$ (for reasons of simplicity, we restrict ourselves only to formulas with one argument):

$$
\begin{aligned}
\|\neg \psi(\sigma)\| & =\|\psi(\sigma)\|^{\prime} \\
\|(\psi \wedge \theta)(\sigma)\| & =\|\psi(\sigma)\| \wedge\|\theta(\sigma)\| \\
\|(\psi \vee \theta)(\sigma)\| & =\|\psi(\sigma)\| \vee\|\theta(\sigma)\| \\
\|(\psi \Rightarrow \theta)(\sigma)\| & =\|\psi(\sigma)\| \Rightarrow\|\theta(\sigma)\| \\
\|(\psi \Leftrightarrow \theta)(\sigma)\| & =\|\psi(\sigma)\| \Leftrightarrow\|\theta(\sigma)\| \\
\|\exists x \psi(x)\| & =\bigvee_{\tau \in V^{\mathbb{B}}}\|\psi(\tau)\| \\
\|\forall x \psi(x)\| & =\bigwedge_{\tau \in V^{\mathbb{B}}}\|\psi(\tau)\| .
\end{aligned}
$$

Now let $G$ be a $\mathbb{B}$-generic ultrafilter over $V$; only the case $G \notin V$ will produce something interesting to us. We define, by recursion on $\rho(\sigma)$, the $G$-interpretation $i_{G}$ of elements of $V^{\mathbb{B}}$ :

$$
i_{G}(\sigma)=\left\{i_{G}(\tau): \tau \in \operatorname{dom} \sigma \wedge \sigma(\tau) \in G\right\} .
$$

We will also write $\sigma_{G}$ instead of $i_{G}(\sigma)$. Finally, let 


$$
V_{\mathbb{B}}[G]=\left\{\sigma_{G}: \sigma \in V^{\mathbb{B}}\right\} .
$$

We call $\left\langle V_{\mathbb{B}}[G],=, \in\right\rangle$ a generic extension of $V$. (We usually need not emphasize the Boolean algebra used in forcing, and write only $V[G]$.) Every $x \in V[G]$ has a "name", i.e. $\bar{x} \in V^{\mathbb{B}}$ such that $i_{G}(\bar{x})=x$.

The canonical names for all elements $x \in V$ (denoted by $\check{x}$ ) are defined by recursion on $\rho(x)$ :

$$
\check{x}=\{\langle\check{y}, 1\rangle: y \in x\} .
$$

We prove, by induction on $\operatorname{rank}(x)$, that $i_{G}(\check{x})=x$. Suppose $i_{G}(\check{y})=y$ for all $y \in V \operatorname{such}$ that $\operatorname{rank}(y)<\operatorname{rank}(x)$. Then

$$
\begin{aligned}
i_{G}(\check{x}) & =\left\{i_{G}(\sigma): \sigma \in \operatorname{dom}(\check{x}) \wedge \check{x}(\sigma) \in G\right\} \\
& =\left\{i_{G}(\check{y}): y \in x \wedge 1 \in G\right\}=\{y: y \in x\}=x .
\end{aligned}
$$

Thus, all $x \in V$ are in $V[G]$ as well. To prove $G \in V[G]$, we find its canonical name as well:

$$
\bar{G}=\{\langle\check{b}, b\rangle: b \in B\} .
$$

Again, we have

$$
\begin{aligned}
i_{G}(\bar{G}) & =\left\{i_{G}(\sigma): \sigma \in \operatorname{dom} \bar{G} \wedge \bar{G}(\sigma) \in G\right\} \\
& =\left\{i_{G}(\check{b}): b \in B \wedge b \in G\right\}=\{b: b \in G\}=G .
\end{aligned}
$$

(Note that this proves part (c) of the Generic Model Theorem below.)

It is convenient here to remind the reader of some facts on absoluteness. There are formulas $\varphi(x)$ of $\mathrm{ZF}(\mathrm{C})$ that can be satisfied by some set in one model, and not be satisfied by that set in another model. Hence classes determined by such formulas differ too; for example the classes of all cardinals need not be the same in two models $\mathcal{M}, \mathcal{N}$ of ZFC, so we write $\operatorname{Card}^{\mathcal{M}}$ and $\operatorname{Card}^{\mathcal{N}}$ to distinguish between those classes. We say that a formula $\varphi(x)$ is absolute for models $\mathcal{M}, \mathcal{N}(\mathcal{M} \subseteq \mathcal{N})$ if for all $x \in \mathcal{M}$

$$
\mathcal{M} \models \varphi(x) \text { iff } \mathcal{N} \models \varphi(x) .
$$

We say that a formula (or the class defined by that formula) is absolute if it is absolute for every two models of set theory. A function $f: X \rightarrow Y$ is absolute if the formula $f(x)=y$ is. For example, natural numbers, the set $\omega$, the function-class rank and the constructible universe $L$ are absolute. 
Theorem 1.30 (The Generic Model Theorem) ([19], Theorem 14.5)

(a) $V[G]$ is a model of ZF.

(b) If $V \models A C$, then $V[G] \models A C$ as well.

(c) $V \subseteq V[G]$ and $G \in V[G]$.

(d) $\operatorname{Ord}^{V[G]}=\operatorname{Ord}^{V}$.

(e) If $\mathcal{N}$ is a transitive model of ZF such that $V \subseteq \mathcal{N}$ and $G \in \mathcal{N}$, then $V[G] \subseteq \mathcal{N}$.

Part (e) of the Generic Model Theorem states that every model obtained by forcing is in certain sense minimal. This is because every set in $V[G]$ can be defined using only $G$ and elements of $V$.

Definition 1.31 A Boolean algebra is atomless if $\forall a \in B^{+} \exists b \in B^{+} b<a$.

Lemma 1.32 (a) If $\mathbb{P}$ is a notion of forcing in $V$ such that

$$
\forall p \in P \exists q, r \in P(q \leq p \wedge r \leq p \wedge q \perp r)
$$

and $G$ is a $\mathbb{P}$-generic filter over $V$, then $G \notin V$.

(b) If $\mathbb{B}$ is a complete Boolean algebra, then $V[G] \neq V$ for every $\mathbb{B}$-generic ultrafilter $G$ over $V$ iff $\mathbb{B}$ is atomless.

Proof. (a) Let $G$ be a filter in $V$ and $D=\{p \in P: p \notin G\}$. D is dense in $\mathbb{P}$ : for every $p \in P$ there are incompatible elements $q$ and $r$ below $p$, and they can not both be in $G$. Clearly $G \cap D=\emptyset$. Thus there is no $\mathbb{P}$-generic filter in $V$.

(b) Let $\mathbb{B}$ be atomless and let $G$ be $\mathbb{B}$-generic. If $p \in B^{+}$, there is $q \in B^{+}$ such that $q<p$. Putting $r=p \wedge q^{\prime}$ we see that (1.1) holds, so by (a) $G \notin V$. Conversely, let there be an element $p \in B^{+}$such that the interval $(0, p)_{\mathbb{B}}$ is empty. $G=\{b \in B: p \leq b\}$ is obviously a $\mathbb{B}$-generic ultrafilter (because every dense set must contain $p$ ). Since $G \in V$, Theorem 1.30(e) implies $V[G]=V$.

Thus, the new model $V[G]$ contains all elements of $V$ and, if $\mathbb{B}$ is atomless, some new sets as well. Hence a set that is not absolute, such as $P(\omega)$, may be different in models $V$ and $V[G]$. In Example 1.1 below new subsets 
of $\omega$ are added by forcing, so $(P(\omega))^{V} \neq(P(\omega))^{V[G]}$.

If $\kappa$ is a cardinal and $X$ a set of cardinality greater than $\kappa$, a bijection $f: \kappa \rightarrow X$ can be added by forcing; this means that a set can change cardinality in a generic extension, so we write $|X|^{V}$ or $|X|^{V[G]}$ to emphasize in which model we are calculating its cardinality. As a special case, when $X=\lambda$ is a cardinal in $V$, as in Example 1.2, it will no longer be a cardinal in $V[G]$; we say that $\lambda$ is collapsed to $\kappa$. If $\operatorname{Card}^{V[G]}=\operatorname{Card}^{V}$ we say that forcing preserves cardinals. If $\operatorname{cf}^{V[G]}(\alpha)=\operatorname{cf}^{V}(\alpha)$ for all limit ordinals $\alpha$, we say that forcing preserves cofinalities.

Lemma 1.33 Let $\kappa$ be a cardinal in $V$. If all regular cardinals $\lambda \leq \kappa$ in $V$ remain regular in $V[G]$, then forcing by $\mathbb{B}$ preserves cardinals less than or equal to $\kappa$, i.e. $\left(\operatorname{Card} \cap \kappa^{+}\right)^{V[G]}=\left(\operatorname{Card} \cap \kappa^{+}\right)^{V}$.

Proof. Let $V[G]$ be a generic extension by $\mathbb{B}$ and suppose that all regular cardinals $\lambda \leq \kappa$ in $V$ remain regular in $V[G]$. If $\lambda \leq \kappa$ is a regular cardinal, then it remains a cardinal by the assumption. Otherwise, $\lambda$ is a limit cardinal, and the set $\operatorname{Reg} \cap \lambda$ is unbounded in $\lambda$ (if $\alpha<\lambda$, then $\alpha<|\alpha|^{+}<\lambda$ and $|\alpha|^{+}$is regular). It follows from our assumption that $(\operatorname{Reg} \cap \lambda)^{V[G]}$ is unbounded in $\lambda$ too. But the supremum of a set of cardinals must be a cardinal too.

It is usually more natural to work with partial orders than with Boolean algebras. In some textbooks the exposition of forcing deals directly with partial orders (for example in [24]). So let $\mathbb{P}$ be a separative partial order. The class $V^{\mathbb{P}}$ can be defined in a similar way as $V^{\mathbb{B}}$ :

$$
\begin{aligned}
& V_{0}^{\mathbb{P}}=\emptyset \\
& V_{\alpha+1}^{\mathbb{P}}=\left\{\sigma: \sigma \subseteq V_{\alpha}^{\mathbb{P}} \times P\right\} \\
& V_{\alpha}^{\mathbb{P}}=\bigcup_{\beta<\alpha} V_{\beta}^{\mathbb{P}} \text { if } \alpha \text { is a limit ordinal }
\end{aligned}
$$

and finally

$$
V^{\mathbb{P}}=\bigcup_{\alpha \in \text { Ord }} V_{\alpha}^{\mathbb{P}}
$$

Now, if $G$ is a $\mathbb{P}$-generic filter, $i_{G}$ and $V[G]$ are defined in an analogous way as above (compare with Lemma 1.28):

$$
i_{G}(\sigma)=\left\{i_{G}(\tau): \exists p \in G\langle\tau, p\rangle \in \sigma\right\}
$$




$$
V[G]=\left\{i_{G}(\sigma): \sigma \in V^{\mathbb{P}}\right\} .
$$

Now let $\mathbb{B}=$ r.o. $(\mathbb{P})$, and let $e: P \rightarrow B^{+}$the canonical embedding. We define $e_{*}: V^{\mathbb{P}} \rightarrow V^{\mathbb{B}}$ by recursion:

$$
e_{*}(\sigma)=\left\{\left\langle e_{*}(\tau), \bigvee_{\langle\tau, p\rangle \in \sigma} e(p)\right\rangle: \tau \in \operatorname{dom} \sigma\right\}
$$

It is easy to prove that $e_{*}$ is maps the class $V^{\mathbb{P}}$ onto $V^{\mathbb{B}}$. If $\mathbb{P}$ has a greatest element 1 , for $x \in V$ we can define the canonical name $\check{x} \in V^{\mathbb{P}}$ in the same way as in $V^{\mathbb{B}}$, and it is easy to prove that $e_{*}(\check{x})=\check{x}$ for all $x \in V$.

For a first-order formula $\varphi, p \in P$ and $\sigma_{1}, \sigma_{2}, \ldots, \sigma_{n} \in V^{\mathbb{P}}$ we define the forcing relation:

$$
p \Vdash_{\mathbb{P}} \varphi\left(\sigma_{1}, \sigma_{2}, \ldots, \sigma_{n}\right) \quad \text { iff } \quad e(p) \leq\left\|\varphi\left(e_{*}\left(\sigma_{1}\right), e_{*}\left(\sigma_{2}\right), \ldots, e_{*}\left(\sigma_{n}\right)\right)\right\| .
$$

We usually omit $\mathbb{P}$ in subscript if it is not necessary to emphasize the notion of forcing that was used. Also, to make formulas more readable, we will write only $\sigma$ instead of $e_{*}(\sigma)$.

\section{Lemma 1.34}

(a) $\|\exists x \in \sigma \varphi(x)\|=\bigvee_{\tau \in \operatorname{dom} \sigma}(\sigma(\tau) \wedge\|\varphi(\tau)\|)$.

(b) $\|\forall x \in \sigma \varphi(x)\|=\bigwedge_{\tau \in \operatorname{dom} \sigma}(\sigma(\tau) \Rightarrow\|\varphi(\tau)\|)$.

Proof. (a)

$$
\begin{aligned}
\|\exists x \in \sigma \varphi(x)\| & =\|\exists x(x \in \sigma \wedge \varphi(x))\| \\
& =\bigvee_{\mu \in V^{\mathbb{B}}}\|\mu \in \sigma \wedge \varphi(\mu)\| \\
& =\bigvee_{\mu \in V^{\mathbb{B}}} \bigvee_{\tau \in \operatorname{dom} \sigma}(\|\tau=\mu\| \wedge \sigma(\tau) \wedge\|\varphi(\mu)\|) \\
& =\bigvee_{\tau \in \operatorname{dom} \sigma}\left(\sigma(\tau) \wedge \bigvee_{\mu \in V^{\mathbb{B}}}(\|\tau=\mu\| \wedge\|\varphi(\mu)\|)\right) \\
& =\bigvee_{\tau \in \operatorname{dom} \sigma}(\sigma(\tau) \wedge\|\exists y(\tau=y \wedge \varphi(y))\|) \\
& =\bigvee_{\tau \in \operatorname{dom} \sigma}(\sigma(\tau) \wedge\|\varphi(\tau)\|) .
\end{aligned}
$$


(b) Follows easily from (a).

In order to simplify writing, in the following lemma we will omit unnecessary variables and identify $e(p)$ with $p$ :

\section{Lemma 1.35}

(a) If $p \Vdash \varphi$ and $q \leq p$, then $q \Vdash \varphi$.

(b) For every $\varphi$ the set $\{q \in P: q \Vdash \varphi$ or $q \Vdash \neg \varphi\}$ is dense in $\mathbb{P}$ (if $q \Vdash \varphi$ or $q \Vdash \neg \varphi$ we say that $q$ decides $\varphi$ ).

(c) $p \Vdash \neg \varphi$ iff $\neg \exists q \leq p q \Vdash \varphi$.

(d) If $\{q \in P: q \Vdash \varphi\}$ is dense below $p$, then $p \Vdash \varphi$.

(e) $p \Vdash \varphi \wedge \psi$ iff $p \Vdash \varphi$ and $p \Vdash \psi$.

(f) $p \Vdash \varphi \vee \psi$ iff $\forall q \leq p \exists r \leq q(r \Vdash \varphi$ or $r \Vdash \psi)$.

(g) If $A$ is a set in $V$, then

$$
p \Vdash \forall x \in \check{A} \varphi(x) \text { iff } \forall a \in A p \Vdash \varphi(\check{a}) .
$$

(h) If $A$ is a set in $V$, then

$$
p \Vdash \exists x \in \check{A} \varphi(x) \text { iff } \forall q \leq p \exists r \leq q \exists a \in A r \Vdash \varphi(\check{a}) .
$$

Proof. (a) and (e) are obvious.

(b) Let $p \in P . p$ must be compatible with at least one of the elements $\|\varphi\|$ and $\|\neg \varphi\|$, say $p \wedge\|\varphi\| \neq 0$. Then there is $q \in P$ such that $q \leq p \wedge\|\varphi\|$; thus $q \leq p$ and $q \Vdash\|\varphi\|$.

(c) Let $p \Vdash \neg \varphi$, and suppose there is $q \leq p$ such that $q \Vdash \varphi$. But by (a) we have $q \Vdash \neg \varphi$ as well, so $q \leq\|\varphi\| \wedge\|\neg \varphi\|=0$, a contradiction. On the other hand, if $\forall q \leq p \neg q \Vdash \varphi$, we have $p \wedge\|\varphi\|=0$ (otherwise there would be an element $q \in P$ below $p \wedge\|\varphi\|$, so $q \Vdash \varphi$ ). But this means that $p \leq\|\varphi\|^{\prime}$, i.e. $p \Vdash \neg \varphi$.

(d) Suppose that $\{q \in P: q \Vdash \varphi\}$ is dense below $p$. If there is $r \leq p$ such that $r \Vdash \neg \varphi$, there would be $q \leq r$ such that $q \Vdash \varphi$, a contradiction. Now (c) implies $p \Vdash \varphi$. 
(f) Using (c) and (e) we get

$$
\begin{aligned}
p \Vdash \varphi \vee \psi & \Leftrightarrow p \Vdash \neg(\neg \varphi \wedge \neg \psi) \\
& \Leftrightarrow \forall q \leq p \neg q \Vdash(\neg \varphi \wedge \neg \psi) \\
& \Leftrightarrow \forall q \leq p \neg(q \Vdash \neg \varphi \wedge q \Vdash \neg \psi) \\
& \Leftrightarrow \forall q \leq p(\neg q \Vdash \neg \varphi \vee \neg q \Vdash \neg \psi) \\
& \Leftrightarrow \forall q \leq p(\exists r \leq q r \Vdash \varphi \vee \exists r \leq q r \Vdash \psi) \\
& \Leftrightarrow \forall q \leq p \exists r \leq q(r \Vdash \varphi \vee r \Vdash \psi) .
\end{aligned}
$$

(g) Using Lemma 1.34 we get

$$
\begin{aligned}
p \Vdash \forall x \in \check{A} \varphi(x) & \Leftrightarrow p \leq\|\forall x \in \check{A} \varphi(x)\| \\
& \Leftrightarrow p \leq \bigwedge_{\tau \in \operatorname{dom} \check{A}}(\check{A}(\tau) \Rightarrow\|\varphi(\tau)\|) \\
& \Leftrightarrow p \leq \bigwedge_{a \in A}(\check{A}(\check{a}) \Rightarrow\|\varphi(\check{a})\|) \\
& \Leftrightarrow p \leq \bigwedge_{a \in A}(1 \Rightarrow \| \varphi(\check{a})) \| \\
& \Leftrightarrow \forall a \in A p \leq\|\varphi(\check{a})\| \\
& \Leftrightarrow \forall a \in A p \Vdash \varphi(\check{a})
\end{aligned}
$$

(h) Using (c) and (g) we get

$$
\begin{aligned}
p \Vdash \exists x \in \check{A} \varphi(x) & \Leftrightarrow p \Vdash \neg \forall x \in \check{A} \neg \varphi(x) \\
& \Leftrightarrow \forall q \leq p \neg q \Vdash \forall x \in \check{A} \neg \varphi(x) \\
& \Leftrightarrow \forall q \leq p \neg \forall a \in A q \Vdash \neg \varphi(\check{a}) \\
& \Leftrightarrow \forall q \leq p \exists a \in A \neg q \Vdash \neg \varphi(\check{a}) \\
& \Leftrightarrow \forall q \leq p \exists a \in A \exists r \leq q r \Vdash \varphi(\check{a}) .
\end{aligned}
$$

One of the implications in part (h) of the previous lemma can be strengthened in a certain sense:

Lemma 1.36 (The Maximum Principle) ([19], Lemma 14.19) If $p \Vdash$ $\exists x \varphi(x)$, then there is $a \in V^{\mathbb{B}}$ such that $p \Vdash \varphi(a)$.

The following statement, given adequate ingredients, provides an element of $V^{\mathbb{B}}$ : 
Lemma 1.37 (The Mixing Lemma) ([19], Lemma 14.18) If $W$ is a set of pairwise disjoint elements of $B$ and $a_{w} \in V^{\mathbb{B}}$ for $w \in W$, then there is $a \in V^{\mathbb{B}}$ such that $\left\|a=a_{w}\right\| \geq w$ for all $w \in W$.

Definition 1.38 Let $\mathbb{P}$ and $\mathbb{Q}$ be partial orders. $i: P \rightarrow Q$ is a complete embedding if:

(1) for $p_{1}, p_{2} \in P$, if $p_{1} \leq p_{2}$ then $i\left(p_{1}\right) \leq i\left(p_{2}\right)$;

(2) for $p_{1}, p_{2} \in P$, if $p_{1} \perp p_{2}$ then $i\left(p_{1}\right) \perp i\left(p_{2}\right)$;

(3) for every $q \in Q$ there is $p \in P$ such that for all $p^{\prime} \leq p \quad i\left(p^{\prime}\right)$ and $q$ are compatible in $\mathbb{Q}$.

(The word "embedding" may be misleading; $i$ is not necessarily one-toone.)

Theorem 1.39 ([24], Theorem VII 7.5) Let $\mathbb{P}$ and $\mathbb{Q}$ be partial orders, and $i: P \rightarrow Q$ a complete embedding in $V$.

(a) If $H$ is a $\mathbb{Q}$-generic filter over $V$, then $i^{-1}[H]$ is a $\mathbb{P}$-generic filter over $V$ and $V\left[i^{-1}[H]\right] \subseteq V[H]$.

(b) If $G$ is a $\mathbb{P}$-generic filter over $V$, then $G^{\prime}=\{q \in Q: \exists p \in G i(p) \leq q\}$ is a $\mathbb{Q}$-generic filter over $V$ and $V[G] \subseteq V\left[G^{\prime}\right]$.

Theorem 1.40 ([18], Lemma 2.7) Let $\mathbb{P}$ and $\mathbb{Q}$ be partial orders, and $h$ : $Q \rightarrow P$ a function such that

(1) for $q_{1}, q_{2} \in Q$, if $q_{1} \leq q_{2}$ then $h\left(q_{1}\right) \leq h\left(q_{2}\right)$;

(2) for every $q \in Q$ and every $p \in P$ such that $p \leq h(q)$ there is $q^{\prime} \in Q$ compatible with $q$ such that $h\left(q^{\prime}\right) \leq p$.

If $H$ is a $\mathbb{Q}$-generic filter over $V$, then $H^{\prime}=\{p \in P: \exists q \in H p \geq h(q)\}$ is a $\mathbb{P}$-generic filter over $V$ and $V\left[H^{\prime}\right] \subseteq V[H]$.

Definition 1.41 Let $\mathbb{P}$ and $\mathbb{Q}$ be partial orders. $i: P \rightarrow Q$ is a dense embedding if

(1) for $p_{1}, p_{2} \in P$, if $p_{1} \leq p_{2}$ then $i\left(p_{1}\right) \leq i\left(p_{2}\right)$;

(2) for $p_{1}, p_{2} \in P$, if $p_{1} \perp p_{2}$ then $i\left(p_{1}\right) \perp i\left(p_{2}\right)$;

(3) $i[P]$ is dense in $\mathbb{Q}$. 
It is easy to see that every dense embedding is complete. The connection between forcing with partial orders and forcing with Boolean algebras can be derived from the next theorem.

Theorem 1.42 ([24], Theorem VII 7.11) Let $\mathbb{P}$ and $\mathbb{Q}$ be partial orders, and $i: P \rightarrow Q$ a dense embedding.

(a) If $H$ is a $\mathbb{Q}$-generic filter over $V$, then $i^{-1}[H]$ is a $\mathbb{P}$-generic filter over $V$ and $V\left[i^{-1}[H]\right]=V[H]$.

(b) If $G$ is a $\mathbb{P}$-generic filter over $V$, then $G^{\prime}=\{q \in Q: \exists p \in G i(p) \leq q\}$ is a $\mathbb{Q}$-generic filter over $V$ and $V[G]=V\left[G^{\prime}\right]$.

As a special case of Theorem 1.42 , if $\mathbb{B}$ is the completion of $\mathbb{P}, F$ is a $\mathbb{P}$-generic filter over $V$ and $G$ the corresponding $\mathbb{B}$-generic ultrafilter (see Lemma 1.28), then we have $V_{\mathbb{P}}[F]=V_{\mathbb{B}}[G]$ and moreover, for every $\sigma \in V^{\mathbb{P}}$, $\sigma_{F}=\left(e_{*}(\sigma)\right)_{G}$ (see [24], Lemma VII 7.13(a)). Hence, forcing with a partial order $\mathbb{P}$ produces the same extension as forcing with r.o. $(\mathbb{P})$ (via the corresponding ultrafilter). Another consequence of the theorem above is that isomorphic partial orders yield the same generic extensions. In general, if for every $\mathbb{P}$-generic filter $G$ there is a $\mathbb{Q}$-generic filter $H$ such that $V_{\mathbb{P}}[G]=V_{\mathbb{B}}[H]$, and vice versa, we will call those partial orders forcing equivalent.

It suffices to investigate only forcing extensions via separative partial orders:

Lemma 1.43 Every partial order $\mathbb{P}$ is forcing equivalent with its separative quotient.

Proof. Follows from Lemma 1.14 and Theorem 1.42.

Finally, we turn to the second fundamental theorem on forcing, disclosing the way to prove various properties of generic extensions.

Theorem 1.44 (The Forcing Theorem) ([19], Theorem 14.6) Let $G$ be a $\mathbb{P}$-generic filter over $V, \mathbb{B}=$ r.o. $(\mathbb{P})$, and let $G^{\prime}$ be the corresponding $\mathbb{B}$ generic ultrafilter over $V$. For every formula $\varphi\left(x_{1}, x_{2}, \ldots, x_{n}\right)$, and every $\sigma_{1}, \sigma_{2}, \ldots, \sigma_{n} \in V^{\mathbb{P}}$ the following conditions are equivalent:

(a) $V[G] \models \varphi\left(i_{G}\left(\sigma_{1}\right), i_{G}\left(\sigma_{2}\right), \ldots, i_{G}\left(\sigma_{n}\right)\right)$;

(b) $\exists p \in G p \Vdash \varphi\left(\sigma_{1}, \sigma_{2}, \ldots, \sigma_{n}\right)$; 
(c) $\left\|\varphi\left(\sigma_{1}, \sigma_{2}, \ldots, \sigma_{n}\right)\right\| \in G^{\prime}$.

Hence, if we choose a suitable partial order $\mathbb{P}$ (and thus the corresponding complete Boolean algebra), we can extend $V$ to a model $V[G]$ having desired properties.

The problem of existence of a generic filter for a partial order $\mathbb{P}$ can be dealt with in several ways. However, we need not work with $V[G]$ itself; if we want to show that some property $\varphi$ is consistent with $\mathrm{ZFC}+\mathrm{A}$, where $\mathrm{A}$ is an additional set of axioms, it is enough to take a notion of forcing $\mathbb{P}$ in a model of ZFC+A and show that $p \Vdash \varphi$ for some $p \in P$ (i.e. $\|\varphi\|>0$ ), and if we want to show that $\varphi$ holds in every extension of $V$, it is enough to prove that $1 \Vdash \varphi$ (that is, $\|\varphi\|=1$ ). Here 1 denotes the greatest element of $\mathbb{P}$. Of course, we may also consider partial orders without one, but adding it does not change r.o. $(\mathbb{P})$ (the completion adds one anyway), so we can assume that $\mathbb{P}$ has a greatest element.

Elements of $V^{\mathbb{P}}$ are also called names for elements of $V[G]$. Particularly useful are so-called "nice names". Now we introduce several such types of names.

Definition 1.45 (a) If $\sigma$ is a name in $V^{\mathbb{B}}$, a $\mathbb{B}$-nice name for a subset of $\sigma$ is any $\tau \in V^{\mathbb{B}}$ of the form $\left\{\left\langle\pi, b_{\pi}\right\rangle: \pi \in \operatorname{dom} \sigma\right\}$, where $b_{\pi} \in B$ for $\pi \in \operatorname{dom} \sigma$.

(b) If $\sigma$ is a name in $V^{\mathbb{P}}$, a $\mathbb{P}$-nice name for a subset of $\sigma$ is any $\tau \in V^{\mathbb{P}}$ of the form $\bigcup\left\{\{\pi\} \times A_{\pi}: \pi \in \operatorname{dom} \sigma\right\}$, where each $A_{\pi}$ is an antichain in $\mathbb{P}$.

Note. Of course, $\tau$ is actually a name for a subset of $\sigma_{G}$, but since we do not know what $\sigma_{G}$ is until $G$ is fixed, we must call $\tau$ "a nice name for a subset of $\sigma "$.

Since there can be no confusion, we will drop the prefixes and call both these types only "nice names". A special case occurs when $x \in V$ (of course, then all $y \in x$ are in $V$ too); then a nice name has the form $\tau=\left\{\left\langle\check{y}, b_{y}\right\rangle: y \in\right.$ $x\}$. In this case $\|\check{y}=\check{z}\|=0$ for different elements $y, z$ in $V$, so $\|\check{y} \in \tau\|=b_{y}$ for all $y \in x$. Nice names for functions will also prove to be very useful to us. 
Definition 1.46 A nice name for a function in $\left({ }^{X} Y\right)^{V[G]}$, where $X$ and $Y$ are sets in $V$, is any $\tau \in V^{\mathbb{B}}$ of the form: $\left\{\left\langle\langle\check{x}, \check{y}\rangle, b_{x, y}\right\rangle: x \in X, y \in Y\right\}$ such that $\left\{b_{x, y}: y \in Y\right\}$ is a maximal antichain in $\mathbb{B}$ for each $x \in X$. The set of all such names will be denoted by $N_{n}\left({ }^{X} Y\right)$.

Lemma 1.47 ([24], Lemma 5.12) If $\sigma, \mu \in V^{\mathbb{P}}$, then there is a nice name $\tau \in V^{\mathbb{P}}$ for a subset of $\sigma$ such that

$$
1 \Vdash(\mu \subseteq \sigma \Rightarrow \mu=\tau) .
$$

It is common practice (and we will follow it) to write down long formulas in a shorter, informal way, as in the lemma below.

Lemma 1.48 If $\mu \in V^{\mathbb{B}}$ is a name in $V[G]$, then there is $\tau \in N_{n}\left({ }^{X} Y\right)$ such that

$$
\| \mu \text { is a function from } \check{\mathrm{X}} \text { to } \check{\mathrm{Y}} \Rightarrow \mu=\tau \|=1 \text {. }
$$

Proof. Without loss of generality we can assume that $X=\kappa$ and $Y=\lambda$ are cardinals in $V$. For all $\alpha<\kappa$ and $0<\beta<\lambda$ let $b_{\alpha, \beta}=\|\langle\check{\alpha}, \check{\beta}\rangle \in$ $\mu \| \backslash \bigvee_{0<\beta_{1}<\beta} b_{\alpha, \beta_{1}}$ and $b_{\alpha, 0}=\left(\bigvee_{0<\beta<\lambda} b_{\alpha, \beta}\right)^{\prime}$. We define $\tau=\left\{\left\langle\langle\check{\alpha}, \check{\beta}\rangle, b_{\alpha, \beta}\right\rangle\right.$ : $\alpha \in \kappa, \beta \in \lambda\}$. Clearly, $\left\{b_{\alpha, \beta}: \beta<\lambda\right\}$ is a maximal antichain for each $\alpha<\kappa$, so $\tau \in N_{n}\left({ }^{\kappa} \lambda\right)$. To prove (1.2), let $G$ be a $\mathbb{B}$-generic ultrafilter over $V$ and (working from now on in $V[G]$ ) assume that $\mu_{G}: \kappa \rightarrow \lambda$. This means that there is $p \in G$ such that

$$
\|\forall \alpha<\check{\kappa} \exists \beta<\check{\lambda}\langle\alpha, \beta\rangle \in \mu\| \geq p
$$

and

$$
\left\|\forall \alpha<\check{\kappa} \neg \exists \beta_{1}, \beta_{2}<\check{\lambda}\left(\beta_{1} \neq \beta_{2} \wedge\left\langle\alpha, \beta_{1}\right\rangle,\left\langle\alpha, \beta_{2}\right\rangle \in \mu\right)\right\| \geq p .
$$

By (1.4) for each $\alpha<\kappa$ and $\beta_{1}<\beta_{2}<\lambda$ we have $\left\|\left\langle\check{\alpha}, \check{\beta}_{1}\right\rangle \in \mu\right\| \wedge \|\left\langle\check{\alpha}, \check{\beta}_{2}\right\rangle \in$ $\mu \| \wedge p=0$, which implies $b_{\alpha, \beta} \wedge p=\|\langle\check{\alpha}, \check{\beta}\rangle \in \mu\| \wedge p$ for $\beta>0$. Furthermore, for each $\alpha$ (1.3) implies $\bigvee_{\beta<\lambda}\|\langle\alpha, \beta\rangle \in \mu\| \geq p$. Both $\left\{b_{\alpha, \beta} \wedge p: \beta<\lambda\right\}$ and $\{\|\langle\check{\alpha}, \breve{\beta}\rangle \in \mu\| \wedge p: \beta<\lambda\}$ are maximal antichains below $p$, so it follows that $b_{\alpha, 0} \wedge p=\|\langle\check{\alpha}, \check{0}\rangle \in \mu\| \wedge p$. Thus $\|\langle\check{\alpha}, \check{\beta}\rangle \in \tau \Leftrightarrow\langle\check{\alpha}, \check{\beta}\rangle \in \mu\| \geq p$ for all $\alpha<\kappa$ and $\beta<\lambda$, so $\|\forall \alpha<\kappa \tau(\alpha)=\mu(\alpha)\| \geq p$ and we get $\tau_{G}=\mu_{G}$.

Later on we will prove a modification of this result (Lemma 2.43). The previous lemma allows us to assume whenever it is convenient, without loss of generality, that a given name for a function belongs to $N_{n}\left({ }^{X} Y\right)$ : 
Corollary 1.49 For any two sets $X, Y \in V$, every function $f: X \rightarrow Y$ in any extension $V[G]$ has a nice name in $V$.

Proof. if $\mu$ is any name for $f$, there is $p \in G$ such that

$$
p \Vdash \mu \text { is a function from } \check{X} \text { to } \check{Y} \text {. }
$$

But from Lemma 1.48 it follows that there is $\tau \in N_{n}\left({ }^{X} Y\right)$ such that

$$
p \Vdash \mu \text { is a function from } \check{X} \text { to } \check{Y} \Rightarrow \mu=\tau,
$$

so $p \Vdash \mu=\tau$. Hence $\tau$ is also a name for $f$.

We conclude this section with some notes about forcing with a product of Boolean algebras.

Lemma 1.50 Let $\left\{\mathbb{B}_{\alpha}: \alpha<\kappa\right\}$ be a family of complete Boolean algebras and $\mathbb{B}=\prod_{\alpha<\kappa} \mathbb{B}_{\alpha}$ (with operations defined componentwise).

(a) If $G$ is a $\mathbb{B}$-generic ultrafilter over $V$ then there is $\alpha<\kappa$ and a $\mathbb{B}_{\alpha^{-}}$ generic ultrafilter $H$ over $V$ such that $V_{\mathbb{B}}[G]=V_{\mathbb{B}_{\alpha}}[H]$.

(b) If $H$ is a $\mathbb{B}_{\alpha}$-generic ultrafilter over $V$ for some $\alpha<\kappa$ then there is a $\mathbb{B}$-generic ultrafilter $G$ over $V$ such that $V_{\mathbb{B}}[G]=V_{\mathbb{B}_{\alpha}}[H]$.

Proof. To simplify the explanation, we give a proof of the special case $\kappa=2$ (the proof in general is similar). For $a \in B$ we denote by $\mathbb{B}_{a}$ the Boolean algebra $\left\langle a \downarrow, \wedge_{a}, \vee_{a}{ }^{\prime a}{ }^{a}, 0, a\right\rangle$, where $\wedge_{a}, \vee_{a}{ }^{\prime}{ }^{\prime a}$ are restrictions of the operations in $\mathbb{B}$ to $a \downarrow$. First we note that, if $\mathbb{B}=\mathbb{B}_{1} \times \mathbb{B}_{2}$, then there are $b_{1}=\left\langle 1_{\mathbb{B}_{1}}, 0_{\mathbb{B}_{2}}\right\rangle$ and $b_{2}=\left\langle 0_{\mathbb{B}_{1}}, 1_{\mathbb{B}_{2}}\right\rangle$ in $\mathbb{B}$ such that $\mathbb{B}_{b_{1}} \cong \mathbb{B}_{1}$ and $\mathbb{B}_{b_{2}} \cong \mathbb{B}_{2}$. By Theorem 1.42 isomorphic Boolean algebras yield the same generic extensions, so it suffices to give proof for $\mathbb{B}_{b_{1}}$ and $\mathbb{B}_{b_{2}}$ instead of $\mathbb{B}_{1}$ and $\mathbb{B}_{2}$.

(a) Let $G$ be a $\mathbb{B}$-generic ultrafilter. Then $G$ contains exactly one of the elements $b_{1}$ and $b_{2}$, for example $b_{1}$. Let $H=\left\{g \wedge b_{1}: g \in G\right\}$. Clearly, $H$ is a $\mathbb{B}_{b_{1}}$-generic ultrafilter. Since $H$ can be defined within $V[G]$, it follows that $H \in V[G]$, so by minimality of $V[H]$ (Theorem $1.30(\mathrm{e})$ ) we get $V[H] \subseteq V[G]$. Moreover, $G=\left\{g \in B: g \wedge b_{1} \in H\right\}$ so $G \in V[H]$. It follows that $V[G] \subseteq V[H]$ as well.

(b) Let $H$ be a $\mathbb{B}_{b_{1}}$-generic ultrafilter. Then $G=\left\{g \in B: g \wedge b_{1} \in H\right\}$ is a $\mathbb{B}$-generic ultrafilter and $H=\left\{g \wedge b_{1}: g \in G\right\}$, so $V[G]=V[H]$ follows as in (a). 


\subsection{Chain conditions and distributivity}

In this section some connections will be given between various properties of partial orders and the effect they have on generic models produced by them.

Theorem 1.51 ([19], Theorem 15.3) If $\kappa$ is a regular cardinal and $\mathbb{P}$ satisfies the $\kappa$-c.c. then $\kappa$ is a regular cardinal in every extension by $\mathbb{P}$ as well.

Corollary 1.52 Every cardinal greater than or equal to c.c.(P) remains a cardinal in every extension by $\mathbb{P}$.

Proof. Similar to the proof of Lemma 1.33, using Theorem 1.51.

The following lemma establishes a chain condition for many important notions of forcing.

Lemma 1.53 ([24], Lemma VII 6.10) Let $\lambda$ be an infinite cardinal and let $I$ and $J$ be arbitrary sets. If

$$
P=\left\{p: p \text { is a function } \wedge \operatorname{dom} p \in[I]^{<\lambda} \wedge \operatorname{ran} p \subseteq J\right\},
$$

then $\langle P, \supseteq\rangle$ satisfies the $\left(|J|^{<\lambda}\right)^{+}-$c.c.

Another way to prove that certain cardinals are preserved in generic extensions is by generalized distributivity. This notion will also be one of the most important in following chapters.

Definition 1.54 Let $\kappa$ and $\lambda$ be infinite cardinals. A complete Boolean algebra $\mathbb{B}$ is $(\kappa, \lambda)$-distributive if for every collection $\left\langle a_{\alpha \beta}: \alpha<\kappa, \beta<\lambda\right\rangle$ of elements of $B$

$$
\bigwedge_{\alpha<\kappa} \bigvee_{\beta<\lambda} a_{\alpha \beta}=\bigvee_{f: \kappa \rightarrow \lambda} \bigwedge_{\alpha<\kappa} a_{\alpha f(\alpha)}
$$

A complete Boolean algebra $\mathbb{B}$ is $\kappa$-distributive if it is $(\kappa, \lambda)$-distributive for all infinite $\lambda \in$ Card.

It is easy to see that the distributivity laws $\bigwedge_{\alpha<\kappa}\left(a \vee a_{\alpha}\right)=a \vee \bigwedge_{\alpha<\kappa} a_{\alpha}$ and $\bigvee_{\alpha<\kappa}\left(a \wedge a_{\alpha}\right)=a \wedge \bigvee_{\alpha<\kappa} a_{\alpha}$ hold in every complete Boolean algebra. Also, the inequality $\geq$ always holds in (1.5): for every $\alpha<\kappa$ and every $f: \kappa \rightarrow \lambda$ we have $a_{\alpha f(\alpha)} \leq \bigvee_{\beta<\lambda} a_{\alpha \beta}$ (since $a_{\alpha f(\alpha)}$ is among elements $a_{\alpha \beta}$ on the right-hand side). It follows that $\bigwedge_{\alpha<\kappa} a_{\alpha \beta} \leq \bigwedge_{\alpha<\kappa} \bigvee_{\beta<\lambda} a_{\alpha \beta}$, and since 
it holds for every $f: \kappa \rightarrow \lambda$, we have $\bigvee_{f: \kappa \rightarrow \lambda} \bigwedge_{\alpha<\kappa} a_{\alpha \beta} \leq \bigwedge_{\alpha<\kappa} \bigvee_{\beta<\lambda} a_{\alpha \beta}$

If $\mathbb{B}$ is a complete Boolean algebra, let us define

$$
\mathfrak{h}_{2}(\mathbb{B})=\min \{\kappa: \mathbb{B} \text { is not }(\kappa, 2) \text {-distributive }\} \text {. }
$$

Definition 1.55 A partition of an element $b$ of a partial order $\mathbb{P}$ is a maximal antichain of elements below $b$. A partition $W_{1}$ is a refinement of a partition $W_{2}$ if for every $x \in W_{1}$ there is $y \in W_{2}$ such that $x \leq y$. A partition of unity 1 of a Boolean algebra $\mathbb{B}$ is also called a partition of $\mathbb{B}$.

Theorem 1.56 The following conditions are equivalent for every complete Boolean algebra $\mathbb{B}$ in $V$ and every infinite cardinal $\kappa$ :

(a) $\mathbb{B}$ is $\kappa$-distributive;

(b) every intersection of $\kappa$ sets open dense in $B$ is open dense;

(c) for every generic extension $V_{\mathbb{B}}[G]$ and every set $A$ in $V$, every function $f: \kappa \rightarrow A$ in $V_{\mathbb{B}}[G]$ is also in $V$

(d) for every $a \in B^{+}$and every collection $\left\{W_{\alpha}: \alpha<\kappa\right\}$ of partitions of $a$ there are $b \in B^{+}$and $w_{\alpha} \in W_{\alpha}$ such that $b \leq w_{\alpha}$ for all $\alpha<\kappa$;

(e) for every $a \in B$ every collection of $\kappa$ partitions of $a$ has a common refinement.

Proof. $\quad(\mathrm{a}) \Rightarrow(\mathrm{b})$ Let $D_{\alpha}$ for $\alpha<\kappa$ be open dense subsets of $\mathbb{B}$. The intersection $D=\bigcap_{\alpha<\kappa} D_{\alpha}$ is always open: if $d \in D$ and $b \leq d$, since every $D_{\alpha}$ is open, $d \in D_{\alpha}$ implies $b \in D_{\alpha}$, so $b \in D$.

Now let $\mathbb{B}$ be $\kappa$-distributive. To prove that $D$ is dense, let $b \in B^{+}$and let us find $d \in D$ such that $d \leq b$. Let $\lambda=\sup _{\alpha<\kappa}\left|D_{\alpha}\right|$. For every $\alpha<\kappa$ let $\left\{a_{\alpha, \beta}: \beta<\kappa_{\alpha}\right\}$ be an enumeration of the set $\left\{b \wedge d: d \in D_{\alpha}\right\}$, and $a_{\alpha, \beta}=0$ for $\kappa_{\alpha} \leq \beta<\lambda$. Since

$$
\bigvee_{\beta<\lambda} a_{\alpha, \beta}=b \wedge \bigvee D_{\alpha}=b
$$

the left-hand side of (1.5) equals $b$. It follows that $\bigvee_{f: \kappa \rightarrow \lambda} c_{f}=b$ as well, where $c_{f}=\bigwedge_{\alpha<\kappa} a_{\alpha, f(\alpha)}$ for $f \in{ }^{\kappa} \lambda$. Hence at least one of the elements $c_{f}$ is nonzero so, since $c_{f} \leq a_{\alpha, f(\alpha)} \in D_{\alpha}$ for all $\alpha<\kappa, c_{f}$ is the element we wanted. 
(b) $\Rightarrow(\mathrm{c})$ Let $V_{\mathbb{B}}[G]$ be a generic extension, $A \in V$ and $f: \kappa \rightarrow A$ a function in $V[G]$. Let $\bar{f}$ be a name for $f$. There is $p_{0} \in G$ such that

$$
p_{0} \Vdash \bar{f} \text { is a function } \wedge \operatorname{dom} \bar{f}=\check{\kappa} \wedge \operatorname{ran} \bar{f} \subseteq \check{A} \text {. }
$$

For each $\alpha<\kappa$ let

$$
D_{\alpha}=\left\{p \leq p_{0}: \exists x \in A p \Vdash \bar{f}(\check{\alpha})=\check{x}\right\} .
$$

We prove that $D_{\alpha}$ is open dense below $p_{0}$ for every $\alpha<\kappa$ : it is obvious that $D_{\alpha}$ is open, so let $q \leq p_{0}$. (1.6) implies that $p_{0} \Vdash \exists x \in \check{A} \bar{f}(\check{\alpha})=x$, i.e.

$$
\forall q \leq p_{0} \exists p \leq q \exists x \in A p \Vdash \bar{f}(\check{\alpha})=\check{x},
$$

so there is $p \leq q$ such that $p \in D_{\alpha}$.

It is easy to see that (b) implies that the intersection of every family of $\kappa$ sets open dense below $p_{0}$ is open dense too. Applying this to the set $D=\bigcap_{\alpha<\kappa} D_{\alpha}$, we obtain $p \in D \cap G$. Now we can define a function $g: \kappa \rightarrow A$ in $V$ : for every $\alpha<\kappa$ let $g(\alpha)=x_{\alpha} \in A$ be such that $p \Vdash \bar{f}(\check{\alpha})=\check{x_{\alpha}}$. But $g(\alpha)=f(\alpha)$ for all $\alpha<\kappa$, so $f=g$.

(c) $\Rightarrow$ (d) First let us notice that we can assume $a=1$ without loss of generality. This is because, if $\left\{W_{\alpha}: \alpha<\kappa\right\}$ is a collection of partitions of $a$, then $\left\{W_{\alpha} \cup\left\{a^{\prime}\right\}: \alpha<\kappa\right\}$ is a collection of partitions of $\mathbb{B}$, and any partition refining it can be reduced to a partition refining $\left\{W_{\alpha}: \alpha<\kappa\right\}$ by excluding all elements below $a^{\prime}$.

Thus, let $\left\{W_{\alpha}: \alpha<\kappa\right\}$ be a collection of partitions of unity, and let $G$ be any $\mathbb{B}$-generic ultrafilter. For every $\alpha<\kappa$ let $f_{\alpha} \in V^{\mathbb{B}}$ be such that $\left\|f_{\alpha}=\check{w}\right\|=w$ for $w \in W_{\alpha}$ (such elements exist by Lemma 1.37). Since $\left\|\exists w \in \check{W}_{\alpha} f_{\alpha}=w\right\|=\bigvee_{w \in W_{\alpha}} w=1$, we have $i_{G}\left(f_{\alpha}\right) \in W_{\alpha}$. In $V[G]$ we define a function $f: \kappa \rightarrow B$ in the following way: let $f(\alpha)=i_{G}\left(f_{\alpha}\right)$. By (c) this function is in $V$, so $\operatorname{ran} f \in V$ as well. For every $\alpha<\kappa$ there is $w \in W_{\alpha}$ such that $\left\|f_{\alpha}=\check{w}\right\|=w \in G$, so $f_{\alpha}=w$ and we get $f(\alpha) \in G$. Hence $b=\bigwedge \operatorname{ran} f \in G$ as well, so $b \neq 0$. Since $f(\alpha) \in W_{\alpha}$ for every $\alpha<\kappa$, we found $b \in B$ such that $b$ is below some element of every $W_{\alpha}$.

$(d) \Rightarrow(e)$ As above, it suffices to show that every collection of $\kappa$ partitions of unity has a common refinement. Thus let $\left\{W_{\alpha}: \alpha<\kappa\right\}$ be a collection of partitions of unity. Let $C=\left\{b \in B: \forall \alpha<\kappa \exists w_{\alpha} \in W_{\alpha} b \leq w_{\alpha}\right\}$, and let $U$ be a maximal antichain in $C$. Suppose $\bigvee U \neq 1$. If we apply (d) to $a=(\bigvee U)^{\prime}$ and the collection $\left\{W_{\alpha}^{\prime}: \alpha<\kappa\right\}$, where $W_{\alpha}^{\prime}=\{w \wedge a: w \in$ $\left.W_{\alpha}\right\} \backslash\{0\}$, we find an element $b \in B$ and $w_{\alpha} \in W_{\alpha}^{\prime}$ such that $b \leq w_{\alpha}$ for $\alpha<\kappa$. This means that $b$ is in $C$, and it is incompatible with all elements 
of $U$, a contradiction with the maximality of $U$. Hence $U$ is a common refinement for all partitions $W_{\alpha}$.

(e) $\Rightarrow$ (a) Let $\lambda$ be an infinite cardinal and let $\left\{a_{\alpha \beta}: \alpha<\kappa, \beta<\lambda\right\}$ be a family of elements of $B$. We already showed that the inequality $\geq$ in (1.5) always holds, so let $u=\bigwedge_{\alpha<\kappa} \bigvee_{\beta<\lambda} a_{\alpha \beta}$. We construct the collection $\left\{b_{\alpha \beta}: \alpha<\kappa, \beta<\lambda\right\}$ such that, for every $\alpha<\kappa, W_{\alpha}=\left\{b_{\alpha \beta}: \beta<\lambda\right\}$ is a partition of $u$ and $b_{\alpha \beta}<a_{\alpha \beta}$ for all $\beta<\lambda$, by recursion on $\beta$ : if we already defined $b_{\alpha \gamma}$ for $\gamma<\beta$ let $b_{\alpha \beta}=a_{\alpha \beta} \wedge\left(\bigvee_{\gamma<\beta} b_{\alpha \gamma}\right)^{\prime} \wedge u$. Since $\bigvee_{\beta<\lambda} a_{\alpha \beta} \geq u$ for every $\alpha<\kappa$, we have $\bigvee_{\beta<\lambda} b_{\alpha \beta}=u$. Now we apply (e) to the collection $\left\{W_{\alpha}: \alpha<\kappa\right\}$ and get a partition $W$ of $u$ such that for every $w \in W$ there is $f_{w}: \kappa \rightarrow \lambda$ such that $b_{\alpha f_{w}(\alpha)} \in W_{\alpha}$ and $w \leq b_{\alpha f_{w}(\alpha)}$ for every $\alpha<\kappa$. This means that $\bigwedge_{\alpha<\kappa} b_{\alpha f_{w}(\alpha)} \geq w$, so $\bigvee_{f: \kappa \rightarrow \lambda} \bigwedge_{\alpha<\kappa} b_{\alpha f(\alpha)} \geq \bigvee_{w \in W} \bigwedge_{\alpha<\kappa} b_{\alpha f_{w}(\alpha)} \geq \bigvee_{w \in W} w=u$

Each of the conditions of Theorem 1.56 except (a) makes sense for partial orders in general as well, and each of them can be used to define $\kappa$ distributivity of partial orders (the usual way to do this is using (b); sometimes such partial orders are called $\kappa$-Baire). It is not hard to prove the result that supports this:

Lemma 1.57 Let $(*)$ be any of the conditions (b), (c), (d) or (e) of Theorem 1.56. A partial order $\mathbb{P}$ has the property $(*)$ iff the Boolean algebra r.o. $(\mathbb{P})$ has $(*)$.

The following corollary of Theorem $1.56(\mathrm{~d})$ and Lemma 1.33 is among the most useful theorems in theory of forcing:

Corollary 1.58 If a partial order $\mathbb{P}$ is $\nu$-distributive for all $\nu<\kappa$, then

(a) if $\operatorname{cf}^{V}(\mu)=\lambda \leq \kappa$, then $\operatorname{cf}^{V[G]}(\mu)=\lambda$ as well;

(b) $\operatorname{Card}^{V} \cap \kappa^{+}=\operatorname{Card}^{V[G]} \cap \kappa^{+}$.

Most of the equivalents in Theorem 1.56 generalize to $(\kappa, \lambda)$-distributivity:

Theorem 1.59 The following conditions are equivalent for every Boolean algebra $\mathbb{B}$ in $V$ and every two cardinals $\kappa$ and $\lambda$ :

(a) $\mathbb{B}$ is $(\kappa, \lambda)$-distributive;

(b) for every $a \in B^{+}$and every collection $\left\{W_{\alpha}: \alpha<\kappa\right\}$ of partitions of $a$ of size at most $\lambda$ there are nonzero $b \leq a$ and $w_{\alpha} \in W_{\alpha}$ such that $b \leq w_{\alpha}$ for all $\alpha<\kappa ;$ 
(c) for every $a \in B$ every collection of $\kappa$ partitions of $a$ of size at most $\lambda$ has a common refinement;

(d) for every generic extension $V_{\mathbb{B}}[G]$, every function $f: \kappa \rightarrow \lambda$ in $V_{\mathbb{B}}[G]$ is also in $V$.

Unlike $\kappa$-distributivity, we can not use conditions (b) and (c) to define $(\kappa, \lambda)$-distributivity for partial orders. Of course, we can use $(\mathrm{d})$, and a possible alternative non-forcing definition will be given in Section 2.5.

Especially important is part (d) of the previous theorem for $\lambda=2$, since a notion of forcing adds a subset $A \subseteq \kappa$ iff it adds its characteristic function $f: \kappa \rightarrow 2$, because each of these two can be defined from the other.

Lemma 1.60 If a complete Boolean algebra $\mathbb{B}$ is $(\kappa, 2)$-distributive, then it is also $\left(\kappa, 2^{\kappa}\right)$-distributive.

Proof. Let $V[G]$ be a generic extension of $V$, and let $\mathbb{B}$ be $(\kappa, 2)$-distributive. By Theorem 1.59 every function $f: \kappa \rightarrow 2$ in $V[G]$ is also in $V$. But then the same holds for subsets of $\kappa$. It follows that all subsets of $\kappa \times \kappa$ in $V[G]$ are also in $V$; this is because the canonical bijection $\varphi: \kappa \rightarrow \kappa \times \kappa$ generates a bijection $\varphi^{\prime}: P(\kappa) \rightarrow P(\kappa \times \kappa)$, so $P(\kappa)^{V}=P(\kappa)^{V[G]}$ implies $P(\kappa \times \kappa)^{V}=P(\kappa \times \kappa)^{V[G]}$.

Now let $f: \kappa \rightarrow P(\kappa)$ be in $V[G] . f$ can be coded by the set $Y_{f}=\{\langle\alpha, \beta\rangle \in$ $\kappa \times \kappa: \beta \in f(\alpha)\}$. By what we proved above $Y_{f}$ belongs to $V$, and so does $f$, because $f(\alpha)=\{\beta<\kappa:\langle\alpha, \beta\rangle \in Y\}$ for $\alpha<\kappa$. By Theorem $1.59 \mathbb{B}$ is $\left(\kappa, 2^{\kappa}\right)$-distributive.

Next we define a property stronger than distributivity, but often easier to prove.

Definition 1.61 A partial order $\mathbb{P}$ is $\kappa$-closed iff for every ordinal $\gamma<\kappa$ and every descending sequence $\left\langle p_{\alpha}: \alpha<\gamma\right\rangle$ of elements of $P$ there is $p \in P$ such that $p \leq p_{\alpha}$ for all $\alpha<\gamma$.

Lemma 1.62 Every $\kappa^{+}$-closed partial order is $\kappa$-distributive.

Proof. Let $\mathbb{P}$ be $\kappa^{+}$-closed and let $\left\{D_{\alpha}: \alpha<\kappa\right\}$ be a collection of open dense sets. By Theorem 1.56 it suffices to prove that $D=\bigcap_{\alpha<\kappa} D_{\alpha}$ is open dense too. $D$ is obviously open, so let $p \in P$; we want to find $r \in D$ such that $r \leq p$. We construct a sequence $\left\langle p_{\alpha}: \alpha<\kappa\right\rangle$ of elements of $P$ by 
recursion: let $p_{0} \in D_{0}$ be such that $p_{0} \leq p$. If $p_{\beta}$ are already defined for $\beta<\alpha$, we consider two cases: first, if $\alpha=\gamma+1$ we simply let $p_{\alpha} \in D_{\alpha}$ be such that $p_{\alpha} \leq p_{\gamma}$; otherwise, if $\alpha$ is a limit ordinal, since $\mathbb{P}$ is $\kappa$-closed, there is $q \in P$ such that $q \leq p_{\beta}$ for all $\beta<\alpha$, and we let $p_{\alpha} \in D_{\alpha}$ be such that $p_{\alpha} \leq q$. Finally, we let $r$ be an element such that $r \leq p_{\alpha}$ for all $\alpha<\kappa$. $p_{\alpha} \in D_{\alpha}$ implies $r \in D_{\alpha}$ for all $\alpha<\kappa$, so $r \in D$.

Corollary 1.63 Every complete Boolean algebra containing a $\kappa^{+}$-closed dense subset is $\kappa$-distributive.

Now we mention another, even more general, distributivity law.

Definition 1.64 A complete Boolean algebra $\mathbb{B}$ is $(\kappa, \lambda, \mu)$-distributive if for every collection $\left\langle a_{\alpha \beta}: \alpha<\kappa, \beta<\lambda\right\rangle$ of elements of $B$

$$
\bigwedge_{\alpha<\kappa \beta<\lambda} a_{\alpha \beta}=\bigvee_{f: \kappa \rightarrow[\lambda]<\mu} \bigwedge_{\alpha<\kappa} \bigvee_{\beta \in f(\alpha)} a_{\alpha \beta}
$$

$\mathbb{B}$ is weakly $(\kappa, \lambda)$-distributive if it is $(\kappa, \lambda, \omega)$-distributive, and it is weakly $\kappa$-distributive if it is weakly $(\kappa, \lambda)$-distributive for all $\lambda \in$ Card. Weakly $(\omega, \omega)$-distributive Boolean algebras are simply called weakly distributive.

Thus $(\kappa, \lambda)$-distributivity is the same as $(\kappa, \lambda, 2)$-distributivity. Again we have a generalization of Theorem 1.56:

Theorem 1.65 The following conditions are equivalent for every Boolean algebra $\mathbb{B}$ in $V$ :

(a) $\mathbb{B}$ is $(\kappa, \lambda, \mu)$-distributive;

(b) for every $a \in B^{+}$and every collection $\left\{W_{\alpha}: \alpha<\kappa\right\}$ of partitions of $a$ of size at most $\lambda$ there are nonzero $b \leq a$ and $V_{\alpha} \in\left[W_{\alpha}\right]^{<\mu}$ such that $b \leq \bigvee V_{\alpha}$ for all $\alpha<\kappa$;

(c) for every $f: \kappa \rightarrow \lambda$ in $V[G]$ there is $g: \kappa \rightarrow[\lambda]^{<\mu}$ in $V$ such that $f(\alpha) \in g(\alpha)$ for all $\alpha<\kappa$.

Corollary 1.66 Forcing with a weakly distributive Boolean algebra does not add an unbounded real, i.e. a function $f: \omega \rightarrow \omega$ such that there is no $h: \omega \rightarrow \omega$ in $V$ such that $f(n) \leq h(n)$ for all $n \in \omega$. 
Proof. Suppose the opposite, that $\mathbb{B}$ is weakly distributive and forcing with $\mathbb{B}$ adds an unbounded real $f$. By previous theorem there is $g: \omega \rightarrow[\omega]^{<\omega}$ in $V$ such that $f(n) \in g(n)$ for all $n \in \omega$. Let $h(n)=\max g(n)$ for $n \in \omega$; then $g$ bounds $f$.

Lemma 1.67 For every complete Boolean algebra $\mathbb{B}$ the following conditions are equivalent:

(a) $\mathbb{B}$ is $\left(\omega, \omega_{1}, \omega_{1}\right)$-distributive;

(b) $\left\|\left|\check{\omega}_{1}^{V}\right|=\check{\omega}\right\|=0$.

Proof. $\quad(\mathrm{a}) \Rightarrow(\mathrm{b})$ Assume that $\left\|\left|\breve{\omega}_{1}^{V}\right|=\check{\omega}\right\|>0$. Then in some generic extension $V[G]$ there is a bijection $f: \omega \rightarrow \omega_{1}^{V}$. But if $\mathbb{B}$ were $\left(\omega, \omega_{1}, \omega_{1}\right)$ distributive, there would be a function $g: \omega \rightarrow\left[\omega_{1}\right]^{<\omega_{1}}$ in $V$ such that $f(n) \in g(n)$ for all $n \in \omega$. If we let $h(n)=\sup g(n), h: \omega \rightarrow \omega_{1}^{V}$ would be a bijection in $V$, a contradiction.

(b) $\Rightarrow$ (a) Suppose $\mathbb{B}$ is not $\left(\omega, \omega_{1}, \omega_{1}\right)$-distributive. Then there is a generic extension $V[G]$ and a function $f: \omega \rightarrow \omega_{1}^{V}$ in $V[G]$ such that there is no $g: \omega \rightarrow\left[\omega_{1}\right]^{<\omega_{1}}$ in $V$ such that $f(n) \in g(n)$ for all $n \in \omega$. But ran $f$ must be unbounded in $\omega_{1}^{V}$ : otherwise the function $g$ defined by $g(n)=\sup \operatorname{ran} f$ for all $n \in \omega$ would bound $f$, a contradiction. Since $\omega_{1}^{V} \leq \omega_{1}^{V[G]}$ and $\operatorname{cf}^{V[G]}\left(\omega_{1}^{V}\right)=\omega$, it follows that $\left|\omega_{1}^{V}\right|^{V[G]}=\omega$.

\subsection{Proper, Sacks, Laver and ${ }^{\omega} \omega$-bounding notions of forcing}

In this section we consider some other properties a notion of forcing can have. The first of these was introduced by Shelah ${ }^{6}$.

Definition 1.68 A notion of forcing is proper if for every uncountable cardinal $\kappa$, every set $S \subseteq[\kappa]^{\omega}$ that is stationary in the ground model remains stationary in the generic extension.

Every c.c.c. partial order, and every $\omega_{1}$-closed partial order is proper (see [19], Lemmas 31.2 and 31.3).

Lemma 1.69 If $\mathbb{P}$ is a proper notion of forcing, then every set that is stationary in $\omega_{1}$ in the ground model remains stationary in $\omega_{1}$ in every generic extension.

\footnotetext{
${ }^{6}$ Saharon Shelah (b. 1945), Israeli mathematician
} 
Proof. We first prove that, if $C$ is a club in $\left[\omega_{1}\right]^{\omega}$, then $C^{*}=\left\{\alpha<\omega_{1}\right.$ : $\alpha=\bigcup D_{n}$ for some ascending sequence $\left\langle D_{n}: n \in \omega\right\rangle$ in $\left.C\right\}$ is a club in $\omega_{1}$. $C^{*}$ is obviously closed, so let $\alpha<\omega_{1}$. We define a sequence $\left\langle\alpha_{n}: n<\omega\right\rangle$ of ordinals and a sequence $\left\langle D_{n}: n<\omega\right\rangle$ of elements of $C$ by recursion. First let $\alpha_{0}=\alpha$ and let $D_{0} \in C$ be such that $\alpha_{0}+1 \subseteq D_{0}$. For $n>0$ let $\alpha_{n}=\sup D_{n-1}$ and $D_{n} \in C$ be such that $\alpha_{n}+1 \subseteq D_{n}$. Finally, let $D=\bigcup_{n \in \omega} D_{n}$. Since $D=\bigcup_{n \in \omega} \alpha_{n}$ as well, $D$ is an ordinal so $D \in C^{*}$.

Let $S$ be stationary subset of $\omega_{1}$ (in $V$ ). $S$ is also stationary in $\left[\omega_{1}\right]^{\omega}$ : if $C$ is a club in $\left[\omega_{1}\right]^{\omega}, C^{*}$ is a club in $\omega_{1}$ so $S \cap C^{*} \neq \emptyset$, which implies $S \cap C \neq \emptyset$. Thus $S$ is also stationary in $\left[\omega_{1}\right]^{\omega}$ in $V[G]$. Now, if $U$ is a club in $\omega_{1}$ in $V[G]$, $U$ is also a club in $\left[\omega_{1}\right]^{\omega}$ in $V[G]$, so $S \cap U \neq \emptyset$.

Definition 1.70 If $g: \omega \rightarrow \omega$ is a nondecreasing unbounded function, we denote $\mathcal{S}^{g}=\left\{\varphi \in{ }^{\omega}\left([\omega]^{<\omega}\right): \forall n<\omega|\varphi(n)| \leq g(n)\right\}$ and call the elements of $\mathcal{S}^{g}$ slaloms. In particular, if $g(n)=n+1$, we denote $\mathcal{S}^{g}$ by $\mathcal{S}$.

Definition 1.71 A forcing notion $\mathbb{P}$ has the Sacks ${ }^{7}$ property if

$$
1 \Vdash \forall f \in \check{\omega}_{\check{\omega}} \exists \varphi \in\left(\mathcal{S}^{V}\right)^{`} \forall n<\check{\omega} f(n) \in \varphi(n) .
$$

The next lemma shows that it is not necessary to bound the width of "the gates" $\varphi(n)$ of a slalom from Definition 1.71 with $n+1$.

Lemma 1.72 For any nondecreasing unbounded $g: \omega \rightarrow \omega$ a forcing notion $\mathbb{P}$ has the Sacks property iff

$$
1 \Vdash \forall f \in \check{\omega}_{\breve{\omega}} \exists \varphi \in\left(\left(\mathcal{S}^{g}\right)^{V}\right)^{\smile} \forall n<\check{\omega} f(n) \in \varphi(n) .
$$

Proof. $(\Rightarrow)$ Let $g: \omega \rightarrow \omega$ be nondecreasing and unbounded, and let $f: \omega \rightarrow \omega$ be a function in $V[G]$.

Claim. If $\mathbb{P}$ has the Sacks property, then

$$
1 \Vdash \forall h \in \check{\omega}(<\check{\omega} \check{\omega}) \exists \varphi \in\left(\left(\mathcal{S}^{*}\right)^{V}\right)^{\smile} \forall n<\check{\omega} h(n) \in \varphi(n),
$$

where $\mathcal{S}^{*}=\left\{\varphi \in{ }^{\omega}\left(\left[{ }^{<\omega} \omega\right]^{<\omega}\right): \forall n<\omega|\varphi(n)| \leq n+1\right\}$.

Proof. Let $\psi:{ }^{<\omega} \omega \rightarrow \omega$ be a bijection in $V$. If $h: \omega \rightarrow<\omega \omega$, let $h^{\prime}: \omega \rightarrow \omega$ be defined by $h^{\prime}(n)=\psi(h(n))$. Applying (1.8) to $h^{\prime}$ we get $\varphi^{\prime} \in \mathcal{S}^{V}$ such that for all $n<\omega h^{\prime}(n) \in \varphi^{\prime}(n)$. Defining $\varphi \in\left(\mathcal{S}^{*}\right)^{V}$ by

\footnotetext{
${ }^{7}$ Gerald E. Sacks, contemporary American mathematician
} 
$\varphi(n)=\psi^{-1}\left[\varphi^{\prime}(n)\right]$ we obtain that for all $n<\omega h(n) \in \varphi(n)$. This proves the Claim.

For $n \in \omega$ let $x(n)=\min \{m \in \omega: g(m) \geq n+1\}$, and let $h: \omega \rightarrow{ }^{<\omega} \omega$ be defined by $h(n)=f \uparrow(x(n))$. Now, applying the Claim to $h$ we get $\varphi^{\prime} \in\left(\mathcal{S}^{*}\right)^{V}$ such that $h(n) \in \varphi^{\prime}(n)$ for $n<\omega$. Now define $\varphi(m)$ for $m \in \omega$ as follows. If $g(m)=n+1$ (which means $x(n) \leq m<x(n+1)$ ) let $\varphi(m)=$ $\left\{\psi(m): \psi \in \varphi^{\prime}(n+1) \wedge \operatorname{dom} \psi>m\right\}$. Now we have $f(m)=h(n+1)(m) \in$ $\varphi(m)$ (since $\left.h(n+1) \in \varphi^{\prime}(n+1)\right)$. But $\left|\varphi^{\prime}(n+1)\right| \leq n+1=g(m)$ implies $|\varphi(m)| \leq g(m)$; thus we proved (1.8).

$(\Leftarrow)$ Analogous to the first implication.

Definition 1.73 A forcing notion $\mathbb{P}$ is ${ }^{\omega} \omega$-bounding if

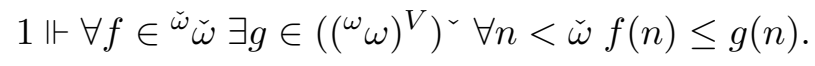

It may seem from Lemma 1.72 that the properties of being ${ }^{\omega} \omega$-bounding and being Sacks are equivalent, but they are not. The reason is that in (1.10) $g$ depends on $f$, and in the formula in Lemma 1.72 the width of $\varphi(n)$ for $\varphi \in \mathcal{S}^{g}$ is given a priori by $g$.

Definition 1.74 A forcing notion $\mathbb{P}$ has the Laver 8 property if

$$
\begin{aligned}
1 \Vdash & \forall f \in \check{\omega}_{\breve{\omega}}\left(\exists h \in\left((\omega \omega)^{V}\right)^{`} \forall n \in \check{\omega} f(n) \leq h(n) \Rightarrow\right. \\
& \left.\exists \varphi \in\left(\mathcal{S}^{V}\right)^{`} \forall n<\check{\omega} f(n) \in \varphi(n)\right) .
\end{aligned}
$$

Clearly, (1.10) and (1.11) imply (1.8), so a partial order has the Sacks property iff it is ${ }^{\omega} \omega$-bounding and has the Laver property. The proof of the following lemma is analogous to the proof of Lemma 1.72 .

Lemma 1.75 For any nondecreasing unbounded $g: \omega \rightarrow \omega$ a forcing notion $\mathbb{P}$ has the Laver property iff

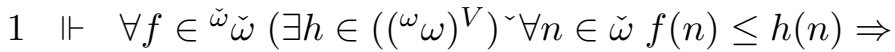

$$
\begin{aligned}
& \left.\exists \varphi \in\left(\left(\mathcal{S}^{g}\right)^{V}\right)^{\sim} \forall n<\check{\omega} f(n) \in \varphi(n)\right) \text {. }
\end{aligned}
$$

\subsection{Various notions of forcing}

In this section we give some examples of notions of forcing, all of which will be used later. We begin with the partial order that was the first ever used for this purpose, introduced by Cohen in [6] and [7].

\footnotetext{
${ }^{8}$ Richard Laver, contemporary American mathematician
} 
Example 1.1 (Cohen forcing) Let $P={ }^{<\omega} 2, P^{\prime}={ }^{<\omega} \omega$ and, $Q=\{f$ : $\left.\operatorname{dom} f \in[\omega]^{<\omega}, \operatorname{ran} f \subseteq\{0,1\}\right\}$. Forcing with $\mathbb{P}=\langle P, \supseteq\rangle$ or $\mathbb{Q}=\langle Q, \supseteq\rangle$ adds a function $f=\bigcup G \in{ }^{\omega} 2$, and forcing with $\mathbb{P}^{\prime}=\left\langle P^{\prime}, \supseteq\right\rangle$ adds a function $g=\bigcup G \in{ }^{\omega} \omega$, called a Cohen real (in both cases $G$ is the corresponding generic filter). But using Theorem 1.42 and the identity function $f: P \rightarrow Q$ we see that the partial orders $\mathbb{P}$ and $\mathbb{Q}$ are forcing equivalent. On the other hand, applying Theorem 1.42 to the bijection $i: P^{\prime} \rightarrow P$ defined by

$$
i\left(\left\langle k_{0}, \ldots, k_{m}\right\rangle\right)=\underbrace{11 \ldots 1}_{k_{0}} 0 \ldots 0 \underbrace{11 \ldots 1}_{k_{m}}
$$

we also obtain that $\mathbb{P}$ and $\mathbb{P}^{\prime}$ are forcing equivalent. Thus all these forcing notions produce the same extensions. Some of the functions in these extensions are unbounded reals.

For any regular cardinal $\kappa, \mathbb{Q}$ can be generalized to $\mathbb{Q}_{\kappa}=\left\langle Q_{\kappa}, \supseteq\right\rangle$, where $Q_{\kappa}=\left\{f: \operatorname{dom} f \in[\kappa]^{<\kappa}, \operatorname{ran} f \subseteq\{0,1\}\right\}$. Forcing with $\mathbb{Q}_{\kappa}$ adds a function $f \in{ }^{\kappa} 2$, i.e. a subset of $\kappa . \mathbb{Q}_{\kappa}$ is $\left(2^{<\kappa}\right)^{+}$-c.c. by Lemma 1.53 , so $\mathbb{Q}$ is c.c.c. Thus it preserves all cardinals and their cofinalities, and so do $\mathbb{P}$ and $\mathbb{P}^{\prime}$.

$\mathbb{Q}_{\kappa}$ can be further generalized to a forcing notion adding a larger number of subsets of $\kappa$. Using this Cohen proved the independence of the Continuum Hypothesis from the axioms of ZFC.

The completion of Cohen notion of forcing (either $\mathbb{P}, \mathbb{P}^{\prime}$ or $\mathbb{Q}$, since these completions are isomorphic) is called the Cohen algebra.

Lemma 1.76 The Cohen algebra is the unique (up to isomorphism) atomless complete Boolean algebra having a countable dense subset.

Another way to obtain the Cohen algebra is the following: let $\mathbb{B}$ be the $\sigma$-algebra of all Borel sets of reals and let $I_{m}$ be the $\sigma$-ideal of meager sets. Then $\mathbb{B} / I_{m} \cong$ r.o. $\left(\left\langle B \backslash I_{m}, \subseteq\right\rangle\right)$, and this algebra is also isomorphic to the Cohen algebra.

Example 1.2 (Collapse forcing) Let $\kappa$ be a regular cardinal and $\lambda>\kappa$ be a cardinal. Let $P=\left\{f: \operatorname{dom} f \in[\kappa]^{<\kappa}\right.$, ran $\left.f \subseteq \lambda\right\}$. The partial order $\langle P, \supseteq\rangle$ is $\kappa$-closed, so by Lemma 1.62 and Corollary 1.58 it preserves all cardinals and cofinalities up to $\kappa$. By Lemma 1.53 it is also $\left(\lambda^{<\kappa}\right)^{+}$-c.c. so, if $\lambda^{<\kappa}=\lambda$, then it preserves the cardinals greater than $\lambda$. But $\lambda$ (and thus all cardinals between $\kappa$ and $\lambda$ ) is collapsed to $\kappa$.

The completion of $\langle P, \supseteq\rangle$ is called a collapsing algebra and denoted $\operatorname{Col}(\kappa, \lambda)$. 
Example 1.3 (Random forcing) This notion of forcing was introduced by Solovay in [31]. Let $\mathbb{B}$ be the $\sigma$-algebra of all Borel sets of reals (or all Borel subsets of the interval $[0,1]$, for example) and let $I_{n}$ be the $\sigma$-ideal of sets of Lebesgue measure zero. Then $\mathbb{B} / I_{n} \cong$ r.o. $\left(\left\langle B \backslash I_{n}, \subseteq\right\rangle\right)$ is a complete Boolean algebra that is not $(\omega, 2)$-distributive, but is weakly $\omega$-distributive.

Forcing via $\mathbb{B}$ also adds a function in ${ }^{\omega} \omega$, called a random real.

Example 1.4 (Shooting a club) This notion of forcing was introduced by Baumgartner ${ }^{9}$, Harrington ${ }^{10}$ and Kleinberg ${ }^{11}$ in [4]. Let $A \subseteq \omega_{1}$ be stationary. We define $P_{A}=\{p \subseteq A: p$ is closed and bounded $\}$ and for $p, q \in P_{A}$

$$
p \leq q \text { iff } q=p \cap \alpha \text { for some } \alpha<\omega_{1} .
$$

$\mathbb{P}_{A}=\left\langle P_{A}, \leq\right\rangle$ is $\omega$-distributive, so by Theorem 1.56 forcing with $\mathbb{P}_{A}$ does not add new countable sets and $\aleph_{1}$ remains a cardinal in $V[G]$. On the other hand, it adds a club subset $C$ of $A$. This can also be a way to destroy a stationary subset of $\omega_{1}$ : if $\omega_{1} \backslash A$ is also stationary in $V$, it does not remain stationary in $V[G]$, because it is disjoint with $C$. In this case r.o. $\left(\mathbb{P}_{A}\right)$ does not have an $\omega_{1}$-closed dense subset, thus providing an example that the reverse implication of Corollary 1.63 does not hold.

Example 1.5 (Prikry forcing) This notion of forcing was constructed by Prikry $^{12}$ in [28]. Let $\kappa$ be a measurable cardinal and $D$ a normal ultrafilter on $\kappa$. Let $P$ be the set of all ordered pairs $\langle s, A\rangle$, where $s$ is a finite increasing sequence of ordinals below $\kappa, A \in D$ and $\min (A)>\max (s)$. The order on $P$ is defined as follows:

$$
\langle t, B\rangle \leq\langle s, A\rangle \text { iff } t \supseteq s \wedge B \subseteq A \wedge \operatorname{ran}(t \backslash s) \subseteq A .
$$

r.o. $(\mathbb{P})$ is $(\omega, \lambda)$-distributive for all $\lambda<\kappa$, but not $(\omega, \kappa)$-distributive. Forcing with $\mathbb{P}$ adds a cofinal function $f: \omega \rightarrow \kappa$ called a Prikry sequence, but it preserves all cardinalities. To prove this Prikry used the following property of $\mathbb{P}$ :

Lemma 1.77 ([19], Lemma 21.12) Let $\mathbb{P}$ be Prikry forcing, $\langle s, A\rangle \in P$ and $\sigma$ a sentence. Then there is $A^{\prime} \in D$ such that $\left\langle s, A^{\prime}\right\rangle \leq\langle s, A\rangle$ and $\left\langle s, A^{\prime}\right\rangle$ decides $\sigma$, meaning that either $\left\langle s, A^{\prime}\right\rangle \Vdash \sigma$ or $\left\langle s, A^{\prime}\right\rangle \Vdash \neg \sigma$.

\footnotetext{
${ }^{9}$ James E. Baumgartner, contemporary American mathematician

${ }^{10}$ Leo A. Harrington, contemporary American mathematician

${ }^{11}$ Eugene Kleinberg, contemporary American mathematician

${ }^{12}$ Karel L. Prikry, contemporary Czech mathematician
} 
Definition 1.78 A partial order $\mathbb{T}=\langle T, \leq\rangle$ is a tree if for every $t \in T$ the set $t \downarrow$ is well-ordered.

We will need some notation regarding trees. If $t \in T$ let

$$
\begin{aligned}
\operatorname{ht}_{\mathbb{T}}(t) & =\text { o.t. }(\mathrm{t} \downarrow) \\
\operatorname{Lev}_{\alpha}(\mathbb{T}) & =\left\{t \in T: \mathrm{ht}_{\mathbb{T}}(t)=\alpha\right\} \\
T_{\alpha} & =\bigcup_{\beta<\alpha} \operatorname{Lev}_{\beta}(\mathbb{T}) \\
\operatorname{ht}(\mathbb{T}) & =\min \left\{\alpha \in \operatorname{Ord}: \operatorname{Lev}_{\alpha}(\mathbb{T})=\emptyset\right\} .
\end{aligned}
$$

We will omit the subscript $\mathbb{T}$ whenever this does not cause any ambiguity.

Definition 1.79 A tree $\mathbb{T}$ will be called:

-a $\kappa$-tree if $\operatorname{ht}(\mathbb{T})=\kappa$ and $\left|\operatorname{Lev}_{\alpha}(\mathbb{T})\right|<\kappa$ for all $\alpha<\kappa$;

-well-pruned if for each $t \in T$ and each $\alpha$ satisfying $h t(t) \leq \alpha<\operatorname{ht}(\mathbb{T})$ there is $s \in \operatorname{Lev}_{\alpha}(\mathbb{T})$ such that $t \leq s$;

-ever-branching if above each $t \in T$ there are at least two incomparable elements;

-normal if: 1) $\mathbb{T}$ has the smallest element;2) $\mathbb{T}$ is well-pruned and 3 ) for all $s, t \in T$, if $\operatorname{ht}(s)=\operatorname{ht}(t)$ is a limit ordinal and $s \downarrow=t \downarrow$, then $s=t$.

Clearly, two elements of a tree $\mathbb{T}=\langle T, \leq\rangle$ are incomparable iff they are incompatible in the reversed tree $\mathbb{T}^{*}=\langle T, \geq\rangle$. A maximal chain in a tree is called a branch.

Example 1.6 (Suslin tree) A $\lambda$-Suslin ${ }^{13}$ tree is a $\lambda$-tree with no chains or antichains of cardinality $\lambda$. An $\omega_{1}$-Suslin tree is called just a Suslin tree. The statement "there are no Suslin trees" is known as the Suslin Hypothesis $(\mathrm{SH})$ and it is neither provable nor refutable in $\mathrm{ZFC}$ : $\neg \mathrm{SH}$ follows from $\diamond$, and $\mathrm{SH}$ from $\neg \mathrm{CH}+\mathrm{MA}_{\aleph_{1}}$. The proofs of these facts can be found in [19], pages 274 and 241 respectively. Forcing with $\mathbb{T}^{*}$ "kills" the Suslin tree $\mathbb{T}$ by adding an uncountable branch to it.

A Suslin algebra is an atomless, c.c.c. $\omega$-distributive complete Boolean algebra. If $\mathbb{T}$ is a normal Suslin tree, then r.o. $\left(\mathbb{T}^{*}\right)$ is a Suslin algebra. Conversely, if $\mathbb{B}$ is a Suslin algebra then $\mathbb{B}$ has a complete subalgebra that is a completion of a reversed Suslin tree.

More on Suslin trees can be found in [33] or [34]. We mention one more fact that will be used later:

\footnotetext{
${ }^{13}$ Mikhail Ya. Suslin (1894-1919), Russian mathematician
} 
Lemma 1.80 For every well-pruned $\kappa^{+}$-Suslin tree $\mathbb{T}=\langle T, \leq\rangle$ the reversed partial order $\mathbb{T}^{*}$ (and therefore the algebra r.o. $\left(\mathbb{T}^{*}\right)$ as well) is $\kappa$-distributive.

Proof. First let us show that for every set $D$ open dense in $\mathbb{T}^{*}$ :

$$
\exists \xi<\kappa^{+} \forall \eta>\xi \operatorname{Lev}_{\eta}(\mathbb{T}) \subseteq D .
$$

Let $A$ be a maximal antichain in $D$. It is also an antichain in $\mathbb{T}^{*}$, so we have $|A| \leq \kappa$. Therefore $\xi=\sup _{a \in A}$ ht $(a)<\kappa^{+}$. Now suppose $t \in T$ is such that $\operatorname{ht}(t)>\xi$. Since $D$ is dense in $\mathbb{T}^{*}$, there is $d \in D$ such that $t \leq d$ and, since $A$ is maximal, there is $a \in A$ comparable to $d$, hence with $t$ too. As $\operatorname{ht}(t)>\xi$ and $\operatorname{ht}(a) \leq \xi$ hold, we also have $a \leq t$, thus $t \in D$. This proves (1.13).

Now assume $D_{\alpha}$, for $\alpha<\kappa$, are open dense subsets of $\mathbb{T}^{*}$. We will prove that $\bigcap_{\alpha<\kappa} D_{\alpha}$ is open dense as well. We already know that the intersection of open sets is always open. For every $D_{\alpha}$ we choose $\xi_{\alpha}$ such that $\forall \eta>$ $\xi_{\alpha} \operatorname{Lev}_{\eta}(\mathbb{T}) \subseteq D_{\alpha}$. If we denote $\xi=\sup _{\alpha<\kappa} \xi_{\alpha}$, the set $\bigcap_{\alpha<\kappa} D_{\alpha}$ contains all levels of the reversed tree $\mathbb{T}^{*}$ below level $L_{\xi}$ so, since $\mathbb{T}$ is well-pruned, it is dense.

Example 1.7 (Sacks forcing) This notion of forcing was introduced by Sacks in [29]. Let $P$ be the set of all initial ever-branching subtrees of the tree ${ }^{<\omega_{2}}$ (they are called perfect trees). Forcing with $\langle P, \supseteq\rangle$ adds $f \in \omega_{2}$ called a Sacks real. This is an example of a partial order satisfying the Sacks property, and hence the Laver property and ${ }^{\omega} \omega$-bounding property as well.

Example 1.8 (Iterated forcing) Sometimes it is necessary to force more than once to obtain a model that satisfies conditions we want to obtain. In that way we get a sequence $\mathcal{M}_{0} \subseteq \mathcal{M}_{1} \subseteq \ldots \subseteq \mathcal{M}_{\gamma} \subseteq \ldots(\gamma<\kappa)$ of models, where each $\mathcal{M}_{\gamma+1}$ is obtained by forcing using a partial order in $\mathcal{M}_{\gamma}$. Actually, we pick these partial orders (more precisely, their names) in $\mathcal{M}_{0}$. There are several ways to obtain $\mathcal{M}_{\gamma}$ for $\gamma \in \operatorname{Lim}$, so there are as many types of iterated forcing: finite support iteration, countable support iteration etc. A method has been developed that allows us to obtain a partial order complex enough so that forcing with it gives the same generic extension as this iteration, see [3], [19] or [24].

Iterated forcing was introduced by Solovay and Tennenbaum ${ }^{14}$ in [32]. Perhaps the most well-known application of this method is the proof of the following theorem.

\footnotetext{
${ }^{14}$ Stanley Tennenbaum, contemporary American mathematician
} 
Theorem 1.81 ([24], Theorem VIII 6.3) Assume that in $V \kappa$ is a regular uncountable cardinal and $2^{<\kappa}=\kappa$. Then there is a partial order $\mathbb{P}$ that is c.c.c. in $V$ and $V[G] \models 2^{\omega}=\kappa \wedge$ MA for every generic extension $V[G]$ by $\mathbb{P}$. 



\section{Chapter 2}

\section{Cut-and-choose games on Boolean algebras}

All the games that will be described in this chapter are played by two players, White and Black, with White playing first. They make their moves subsequently. For each game we will describe the moves each of the players is allowed to make, and the criterion that they need to fulfill to win. We begin with some history, using it to introduce the notions and notation that we will use from now on.

\subsection{Infinite games. The Banach-Mazur game}

The first infinite game was defined in 1930. Today it is known as the $B a$ nach ${ }^{1}-M a z u r^{2}$ game.

Definition 2.1 Let $A \subseteq{ }^{\omega} \omega$. The game $\mathcal{G}_{\mathrm{BM}}(A)$ is played as follows:

- First, White chooses $a_{0} \in{ }^{<\omega} \omega$;

- in his $n$-th move Black chooses $b_{n} \in{ }^{<\omega} \omega$ such that $b_{n} \supset a_{n}$;

- in his $n$-th move (for $n>0$ ) White chooses $a_{n} \in<\omega_{\omega}$ such that $a_{n} \supset b_{n-1}$.

Thus $x=\bigcup_{n<\omega} a_{n}=\bigcup_{n<\omega} b_{n} \in{ }^{\omega} \omega$. White wins iff $x \in A$, and Black wins otherwise.

\footnotetext{
${ }^{1}$ Stefan Banach (1892-1945), Polish mathematician

${ }^{2}$ Stanisław Mazur (1905-1981), Polish mathematician
} 
A sequence $\left\langle a_{0}, b_{0}, \ldots, a_{n}, b_{n}, \ldots\right\rangle$ of moves of both players obtained in this way will be called a play, and each its initial subsequence $\left\langle a_{0}, b_{0}, \ldots, a_{n}\right.$, $\left.b_{n}\right\rangle$ or $\left\langle a_{0}, b_{0}, \ldots, b_{n-1}, a_{n}\right\rangle$ a partial play.

Perhaps the most important notion in infinite game theory is the notion of a winning strategy. Informally speaking, we say that one of the players has a winning strategy in such a game iff there is an algorithm such that, if he plays by that algorithm then he wins the game regardless of the other player's moves. More formally, a strategy for a player X is a function that maps every possible partial play that can precede a move of $\mathrm{X}$ to an element of the set of allowed moves for X. (Of course, $\Sigma$ may be a multi-valued function, but assuming the Axiom of Choice we can restrict our attention only to single-valued functions.)

Thus, in the game described above, if we denote with $S_{n}$ the set of all strictly increasing sequences of length $n$ of elements of $<\omega \omega$, a strategy for White is a function $\Sigma: \bigcup_{n<\omega} S_{2 n} \rightarrow{ }^{<\omega} \omega$ such that, for all $n \in \omega$ and all $\left\langle a_{0}, b_{0}, \ldots, a_{n-1}, b_{n-1}\right\rangle \in S_{2 n}, \Sigma\left(\left\langle a_{0}, b_{0}, \ldots, a_{n-1}, b_{n-1}\right\rangle\right) \supset b_{n-1}$. This means that, if the moves so far were $a_{0}, b_{0}, \ldots, a_{n-1}, b_{n-1}$, then White should play $\Sigma\left(\left\langle a_{0}, b_{0}, \ldots, a_{n-1}, b_{n-1}\right\rangle\right)$. Such a function is a winning strategy if for every play $\left\langle a_{0}, b_{0}, \ldots, a_{n}, b_{n}, \ldots\right\rangle$ in which White follows $\Sigma$ (i.e. $a_{n}=\Sigma\left(\left\langle a_{0}, b_{0}, \ldots, a_{n-1}, b_{n-1}\right\rangle\right)$ for all $\left.n \in \omega\right)$ the sequence $\bigcup_{n<\omega} a_{n}$ be-

longs to $A$. Similarly, a strategy for Black is a function $\Sigma: \bigcup_{n<\omega} S_{2 n+1} \rightarrow$ $<\omega_{\omega}$ such that $\Sigma\left(\left\langle a_{0}, b_{0}, \ldots, b_{n-1}, a_{n}\right\rangle\right) \supset a_{n}$, and it is a winning strategy if for every play $\left\langle a_{0}, b_{0}, \ldots, a_{n}, b_{n}, \ldots\right\rangle$ in which Black follows $\Sigma$ (i.e. $b_{n}=\Sigma\left(\left\langle a_{0}, b_{0}, \ldots, b_{n-1}, a_{n}\right\rangle\right)$ for all $\left.n \in \omega\right) \bigcup_{n<\omega} a_{n} \notin A$ holds.

For every game $\mathcal{G}$ there are three possibilities:

1) White has a winning strategy,

2) Black has a winning strategy or

3) neither of two players has a winning strategy; in this case we say that $\mathcal{G}$ is undetermined.

Notice that, although each play $\left\langle a_{0}, b_{0}, \ldots, a_{n}, b_{n}, \ldots\right\rangle$ is won by one of the players, due to the infinity of the game this doesn't mean that one of them must have a winning strategy. Of course, this may depend on certain parameters, such as the set $A$ in the game $\mathcal{G}_{\mathrm{BM}}(A)$. Trivially, if $A=\omega$ then White wins every play, and if $A=\emptyset$ then Black wins every play. 
We remind the reader that a subset $A$ of a topological space is nowhere dense if the interior of its closure is empty, meager if it is a countable union of nowhere dense sets, and has the Baire property if there is an open set $G$ such that $(A \backslash G) \cup(G \backslash A)$ is meager.

Lemma 2.2 ([19], Lemma 33.7) Let $A \subseteq{ }^{\omega} \omega$. Black has a winning strategy in the game $\mathcal{G}_{\mathrm{BM}}(A)$ iff $A$ is meager in the usual topology.

For $\varphi \in{ }^{<\omega} \omega$ let us denote $B_{\varphi}=\left\{x \in{ }^{\omega} \omega: \varphi \subseteq x\right\}$ - a base open set in the usual topology on ${ }^{\omega} \omega$.

Lemma 2.3 ([19], Corollary 33.8) Let $A \subseteq{ }^{\omega} \omega$. White has a winning strategy in the game $\mathcal{G}_{\mathrm{BM}}(A)$ iff for some $\varphi \in{ }^{<\omega} \omega$ the set $B_{\varphi} \backslash A$ is meager in the usual topology.

The Banach-Mazur game can be reformulated as a game of the following form.

Definition 2.4 If $X \subseteq{ }^{\omega} \omega$, the game $\mathcal{G}^{\prime}(X)$ is played as follows. The players, White and Black, subsequently choose natural numbers $a_{0}, b_{0}, \ldots$, $a_{n}, b_{n}, \ldots$; White wins if the sequence $\left\langle a_{0}, b_{0}, \ldots, a_{n}, b_{n}, \ldots\right\rangle$ is in $X$, and Black wins otherwise.

Now let $f: \omega \rightarrow<\omega \omega$ be a bijection. The Banach-Mazur game played on a set $A$ can be rephrased as follows: instead of a finite sequence $x$ of natural numbers each player chooses a natural number $f^{-1}(x)$ in each move. Let $X$ be the set of all sequences $\left\langle a_{0}, b_{0}, \ldots, a_{n}, b_{n}, \ldots\right\rangle \in{ }^{\omega} \omega$ such that either $f\left(a_{n}\right) \not \subset f\left(b_{n}\right)$ for some $n \in \omega$, or the sequence $\left\langle f\left(a_{0}\right), f\left(b_{0}\right), \ldots, f\left(a_{n}\right)\right.$, $\left.f\left(b_{n}\right), \ldots\right\rangle$ is strictly increasing and $\bigcup_{n \in \omega} f\left(a_{n}\right) \in A$. Then White wins a play $\left\langle a_{0}, b_{0}, \ldots, a_{n}, b_{n}, \ldots\right\rangle$ of the game $\mathcal{G}^{\prime}(X)$ iff White wins the play $\left\langle f\left(a_{0}\right), f\left(b_{0}\right), \ldots, f\left(a_{n}\right), f\left(b_{n}\right), \ldots\right\rangle$ of the game $\mathcal{G}_{\mathrm{BM}}(A)$.

The following additional axiom, defined by Mycielski ${ }^{3}$ and Steinhaus ${ }^{4}$, is known as the Axiom of Determinacy.

(AD) For every set $X \subseteq{ }^{\omega} \omega$ the game $\mathcal{G}^{\prime}(X)$ is determined.

So if $\mathrm{AD}$ holds, the Banach-Mazur game $\mathcal{G}_{\mathrm{BM}}(A)$ is determined for every $A \subseteq{ }^{\omega} \omega$ as well. On the other hand, the Axiom of Choice implies that there

\footnotetext{
${ }^{3}$ Jan Mycielski (b. 1932), Polish mathematician

${ }^{4}$ Hugo D. Steinhaus (1887-1972), Polish mathematician
} 
is a set of reals that does not have the Baire property (for the construction of such a set see [14], page 2). Thus the following lemma, which is a direct consequence of Corollary 27.4 from [23], implies that in ZFC the BanachMazur game can be undetermined.

Lemma 2.5 If $A$ is a set that does not have the Baire property, then there is an open set $O_{A} \subseteq{ }^{\omega} \omega$ such that the game $\mathcal{G}_{\mathrm{BM}}\left(A \backslash O_{A}\right)$ is undetermined.

More information on the games of type $\mathcal{G}^{\prime}(X)$ and the Axiom of Determinacy can be found in [23] or [19].

\subsection{Games on Boolean algebras. The game $\mathcal{G}_{\text {inf }}(\kappa)$}

From now on we will concentrate on games characterizing certain important properties of complete Boolean algebras. The first of these games, inspired by the Banach-Mazur game, was introduced by $\mathrm{Jech}^{5}$ in [15]. It can be defined on any partial order, so let $\mathbb{P}=\langle P, \leq\rangle$ be a partial order and let $\mathbb{B}=$ r.o. $(\mathbb{P})$ be its completion. Unless otherwise stated, all results in this section are due to Jech.

Definition 2.6 The descending chain game $\mathcal{G}_{\text {inf }}(\omega)$ is played on a partial order $\mathbb{P}$ as follows:

- first, White chooses $a_{0} \in P$;

- in his $n$-th move Black chooses $b_{n} \in P$ such that $b_{n} \leq a_{n}$;

- in his $n$-th move (for $n>0$ ) White chooses $a_{n} \in P$ such that $a_{n} \leq b_{n-1}$.

Black wins if there is $p \in P$ such that $p \leq a_{n}$ for all $n \in \omega$ (this also means: $p \leq b_{n}$ for all $\left.n \in \omega\right)$, and White wins otherwise.

Thus, if we denote with $D\left({ }^{n} P\right)$ the set of all descending sequences of length $n$ in $P$, in the game $\mathcal{G}_{\text {inf }}(\omega)$, a strategy for White is a function $\Sigma: \bigcup_{n<\omega} D\left({ }^{2 n} P\right) \rightarrow P$ such that $\Sigma\left(\left\langle a_{0}, b_{0}, \ldots, a_{n}, b_{n}\right\rangle\right) \leq b_{n}$ for every partial play $\left\langle a_{0}, b_{0}, \ldots, a_{n}, b_{n}\right\rangle$. Such a strategy is a winning strategy if for every play $\left\langle a_{0}, b_{0}, \ldots, a_{n}, b_{n}, \ldots\right\rangle$ in which White follows $\Sigma$ there is no $p \in P$ such that $p \leq b_{n}$ for all $n<\omega$. A (winning) strategy $\Sigma: \bigcup_{n<\omega} D\left({ }^{2 n+1} P\right) \rightarrow P$ for Black is defined in a similar, quite natural, way.

\footnotetext{
${ }^{5}$ Thomas J. Jech (b. 1944), Czech mathematician
} 
When playing this game on a complete Boolean algebra $\mathbb{B}$, it will be understood that we actually play on $\left\langle B^{+}, \leq\right\rangle$; thus White wins a play $\left\langle a_{0}, b_{0}, \ldots, a_{n}, b_{n}, \ldots\right\rangle$ iff $\bigwedge_{n \in \omega} a_{n}=0$. Now we will need the following obvious lemma.

Lemma 2.7 If $\Sigma: \bigcup_{n<\omega} D\left({ }^{2 n} P\right) \rightarrow P$ is a winning strategy for White in $\mathcal{G}_{\text {inf }}(\omega)$ played on $\mathbb{P}$ and $\Sigma_{1}: \bigcup_{n<\omega} D\left({ }^{2 n} P\right) \rightarrow P$ is such that $\Sigma_{1}(x) \leq \Sigma(x)$ for all $x \in \bigcup_{n<\omega} D\left({ }^{2 n} P\right)$, then $\Sigma_{1}$ is also a winning strategy for White in $\mathcal{G}_{\text {inf }}(\omega)$ played on $\mathbb{P}$.

Lemma 2.8 Let $\mathbb{P}$ be a separative partial order and $\mathbb{B}=$ r.o.($(\mathbb{P})$.

(a) White has a winning strategy in the game $\mathcal{G}_{\text {inf }}(\omega)$ played on $\mathbb{P}$ iff he has a winning strategy in $\mathcal{G}_{\text {inf }}(\omega)$ played on $\mathbb{B}$.

(b) Black has a winning strategy in the game $\mathcal{G}_{\text {inf }}(\omega)$ played on $\mathbb{P}$ iff he has a winning strategy in $\mathcal{G}_{\text {inf }}(\omega)$ played on $\mathbb{B}$.

Proof. We will prove part (a), and part (b) is proved similarly (see [15]).

$(\Rightarrow)$ Let $\Sigma: \bigcup_{n<\omega} D\left({ }^{2 n} P\right) \rightarrow P$ be a winning strategy for White in the game $\mathcal{G}_{\text {inf }}(\omega)$ played on $\mathbb{P}$. We define $\phi: \bigcup_{n<\omega} D\left({ }^{2 n} B\right) \rightarrow \bigcup_{n<\omega} D\left({ }^{2 n} P\right)$ by recursion on the length of a sequence $x \in \bigcup_{n<\omega} D\left({ }^{2 n} B\right)$. Having defined $\phi(y)$ for every sequence $y$ of even length less than $2(n+1)$, and given a sequence $x=\left\langle a_{0}, b_{0}, \ldots, a_{n}, b_{n}\right\rangle \in \bigcup_{n<\omega} D\left({ }^{2 n} B\right)$, let $p=\Sigma(\phi(x \uparrow 2 n))$. Let $q \in P$ be such that $q \leq p \wedge b_{n}$ if $p \wedge b_{n} \neq 0$, and let $q \leq p$ be an arbitrary element of $P$ otherwise. Finally we define $\phi(x)=\phi(x \nmid 2 n)^{\wedge}\langle p, q\rangle$.

Now we define $\Sigma^{\prime}: \bigcup_{n<\omega} D\left({ }^{2 n} B\right) \rightarrow B$ in the following way: $\Sigma^{\prime}(x)=$ $\Sigma(\phi(x))$. Let us show that $\Sigma^{\prime}$ is a winning strategy for White in $\mathcal{G}_{\text {inf }}(\omega)$ played on $\mathbb{B}$. Let $x=\left\langle a_{0}, b_{0}, \ldots, a_{n}, b_{n}, \ldots\right\rangle$ be a play in which White follows $\Sigma^{\prime}$, and let $y=\left\langle p_{0}, q_{0}, \ldots, p_{n}, q_{n}, \ldots\right\rangle=\bigcup_{n \in \omega} \phi\left(x\lceil 2 n)\right.$; it is a play in $\mathcal{G}_{\text {inf }}(\omega)$ since $q_{n} \leq p_{n}$ and $p_{n+1} \leq q_{n}$ for all $n<\omega$. Then in $y$ White follows $\Sigma$ and $p_{n}=\Sigma\left(\phi(x\lceil 2 n))=\Sigma^{\prime}\left(x\lceil 2 n)=a_{n}\right.\right.$ for $n \in \omega$. Since White wins the play $y$ by assumption, he wins $x$ as well: if there is $b \in B^{+}$such that $b \leq a_{n}$ for all $n \in \omega$, then there is also $p \in P$ such that $p \leq b$, and hence $p \leq p_{n}$ for all $n \in \omega$ as well.

$(\Leftarrow)$ Now let $\Sigma: \bigcup_{n<\omega} D\left({ }^{2 n} B\right) \rightarrow B$ be a winning strategy for White in the game $\mathcal{G}_{\text {inf }}(\omega)$ played on $\mathbb{B}$, and let $\Sigma_{1}$ be defined as follows: if $x=$ $\left\langle a_{0}, b_{0}, \ldots, a_{n}, b_{n}\right\rangle \in \bigcup_{n<\omega} D\left({ }^{2 n} B\right)$, let $p=\Sigma_{1}(x)$ be an arbitrary element of $P$ such that $p \leq \Sigma(x)$. By Lemma 2.7, $\Sigma_{1}$ is also a winning strategy for White. Let $\Sigma^{\prime}=\Sigma_{1} \uparrow \bigcup_{n<\omega} D\left({ }^{2 n} P\right)$. Then $\Sigma^{\prime}: \bigcup_{n<\omega} D\left({ }^{2 n} P\right) \rightarrow P$.

In order to prove that $\Sigma^{\prime}$ is a winning strategy for White in $\mathcal{G}_{\text {inf }}(\omega)$ played on $\mathbb{P}$, we take any play $\left\langle a_{0}, b_{0}, \ldots, a_{n}, b_{n}, \ldots\right\rangle$ on $\mathbb{P}$ in which White follows 
$\Sigma^{\prime}$. Then it is also a play on $\mathbb{B}$ in which White follows $\Sigma_{1}$, so White wins. But this means that there is no $b \in B$ such that $b \leq b_{n}$ for all $n \in \omega$, so there is no $p \in P$ that satisfies the same condition either.

Theorem 2.9 White has a winning strategy in $\mathcal{G}_{\text {inf }}(\omega)$ played on $\mathbb{B}$ iff $\mathbb{B}$ is not $\omega$-distributive.

\section{Proof.}

$(\Rightarrow)$ Let $\Sigma$ be a winning strategy for White. Let $a_{0}=\Sigma(\emptyset)$. We define, for each $n \in \omega$, an antichain $W_{n}$ below $a_{0}$ and a subset $P_{n}$ of $D\left({ }^{2 n+1} B\right)$ as follows. First let $W_{0}=\left\{a_{0}\right\}$ and $P_{0}=\left\{\left\langle a_{0}\right\rangle\right\}$. For $n>0$ let $Q_{n}$ be a maximal subset of $B$ such that:

(i) for each $q \in Q_{n}$ there is $x_{q} \in P_{n-1}$ such that, if $p$ is the last element of $x_{q}$, then $q \leq p$;

(ii) $\left.W_{n}=\left\{\Sigma\left(x_{q}\right\urcorner q\right): q \in Q_{n}\right\}$ is an antichain below $a_{0}$.

We define $\left.P_{n}=\left\{x_{q} q^{\urcorner} \Sigma\left(x_{q}\right\urcorner q\right): q \in Q_{n}\right\}$.

Let us prove by induction on $n$ that $W_{n}$ is a maximal antichain below $a_{0}$. Suppose not and let $q^{\prime}=a_{0} \backslash \bigcup W_{n}$. Since $W_{n-1}$ is maximal, there is $r \in W_{n-1}$ such that $r \wedge q^{\prime} \neq 0$. Furthermore, there is $p \in P_{n-1}$ such that $r$ is the last element of $p$. Hence $r \wedge q^{\prime}$ can be added to $Q_{n}$ so that the conditions (i) and (ii) remain true, a contradiction with the maximality of $Q_{n}$.

Thus we obtained a collection $\left\{W_{n}: n \in \omega\right\}$ of partitions of $a_{0}$, such that $W_{n+1}$ is a refinement of $W_{n}$ for $n \in \omega$. Let us show that $\left\{W_{n}: n \in \omega\right\}$ does not satisfy condition $(\mathrm{d})$ of Theorem 1.56 , and it will follow that $\mathbb{B}$ is not $\omega$-distributive. So let $a_{n} \in W_{n}$ for $n \in \omega$, and let $b_{n}(n \in \omega)$ be the unique element of $Q_{n+1}$ that lies between $a_{n}$ and $a_{n+1}$. Then $\left\langle a_{0}, b_{0}, \ldots, a_{n}, b_{n}, \ldots\right\rangle$ is a play in which White follows the strategy $\Sigma$, so there is no $b \in B^{+}$such that $b \leq a_{n}$ for all $n \in \omega$.

$(\Leftarrow)$ Suppose $\mathbb{B}$ is not $\omega$-distributive. By Theorem 1.56 there are $a \in B^{+}$ and a collection $\left\{W_{n}: n \in \omega\right\}$ of partitions of $a$ such that, if $w_{n} \in W_{n}$ for all $n \in \omega$, then $\bigwedge_{n \in \omega} w_{n}=0$. We define a strategy $\Sigma: \bigcup_{n<\omega} D\left({ }^{2 n} B\right) \rightarrow B$ for White: first let $\Sigma(\emptyset)=a$. Let $n>0$ and $\left\langle a_{0}, b_{0}, \ldots, a_{n-1}, b_{n-1}\right\rangle \in D\left({ }^{2 n} B\right)$. There is $w \in W_{n-1}$ such that $b_{n-1} \wedge w \neq 0$, so let $\Sigma\left(\left\langle a_{0}, b_{0}, \ldots, a_{n-1}, b_{n-1}\right\rangle\right)=$ $b_{n-1} \wedge w$. Now it is easy to see that this is a winning strategy: if $\left\langle a_{0}, b_{0}, a_{1}\right.$, $\left.b_{1}, \ldots, a_{n}, b_{n}, \ldots\right\rangle$ is a play in which White obeys $\Sigma$ and we denote by $w_{n}$ the unique element of $W_{n}$ such that $a_{n+1} \leq w_{n}$ for every $n \in \omega$, we have $\bigwedge_{n \in \omega} a_{n} \leq \bigwedge_{n \in \omega} w_{n}=0$.

Theorem 2.10 If a partial order $\mathbb{P}$ has an $\omega_{1}$-closed dense subset, then Black wins $\mathcal{G}_{\text {inf }}(\omega)$ on $\mathbb{P}$. 
Proof. Let $D \subseteq P$ be $\omega_{1}$-closed and dense in $\mathbb{P}$. The winning strategy of Black is defined as follows: let $\Sigma\left(\left\langle a_{0}, b_{0}, \ldots, b_{n-1}, a_{n}\right\rangle\right)$ be any element $b_{n} \in D$ such that $b_{n} \leq a_{n}$. Then since $D$ is $\omega_{1}$-closed, for every play $\left\langle a_{0}, b_{0}, \ldots, a_{n}, b_{n}, \ldots\right\rangle$ in which Black follows $\Sigma$ there is $b \in D$ such that $b \leq b_{n}$ for all $n \in \omega$.

The possibility of reversing this implication was thoroughly investigated, and the following results were obtained by Jech, Foreman ${ }^{6}$ and Veličković ${ }^{7}$ respectively.

Theorem 2.11 ([16]) Black has a winning strategy in $\mathcal{G}_{\text {inf }}(\omega)$ played on a partial order $\mathbb{P}$ iff there exists a partial order $\mathbb{Q}$ such that $\mathbb{P} \times \mathbb{Q}$ has an $\omega_{1}$-closed dense subset.

Theorem 2.12 ([12]) If Black has a winning strategy in $\mathcal{G}_{\text {inf }}(\omega)$ played on a complete Boolean algebra $\mathbb{B}$ and, for some cardinal $\kappa, \mathbb{B}$ is $\kappa$-distributive and has a dense set of cardinality $\kappa^{+}$, then $\mathbb{B}$ has an $\omega_{1}$-closed dense subset.

Theorem 2.13 ([36]) If Black has a winning strategy in $\mathcal{G}_{\text {inf }}(\omega)$ played on a complete Boolean algebra $\mathbb{B}$ and $\mathbb{B}$ has a dense subset of cardinality at most $\mathfrak{c}$, then $\mathbb{B}$ has an $\omega_{1}$-closed dense subset.

It is also possible that none of the players has a winning strategy on a partial order. One such example is the partial order $\mathbb{P}_{A}$ described in Example 1.4. Precisely we have

Lemma 2.14 Let $A \subseteq \omega_{1}$ be such that both $A$ and $\omega_{1} \backslash A$ are stationary. If $\mathbb{P}_{A}$ is the notion of forcing shooting a club, then neither player wins $\mathcal{G}_{\text {inf }}(\omega)$ on $\mathbb{P}_{A}$.

Proof. Suppose first that there is a winning strategy $\Sigma$ for Black. We define, by recursion, functions $a:\left[\omega_{1}\right]^{<\omega} \rightarrow P_{A}$ (showing how White should play) and $b:\left[\omega_{1}\right]^{<\omega} \rightarrow \omega_{1}$ in the following way: first let

$$
\begin{aligned}
a\left(\alpha_{0}\right) & =\text { any } p \in P_{A} \text { such that } \max (p)>\alpha_{0} \\
b\left(\alpha_{0}\right) & =\max \left(a\left(\alpha_{0}\right)\right) .
\end{aligned}
$$

Now, if $\alpha_{0}, \alpha_{1}, \ldots, \alpha_{n} \in \omega_{1}$ are ordinals such that $\alpha_{0}<\alpha_{1}<\ldots<\alpha_{n}$, define $a\left(\alpha_{0}, \alpha_{1}, \ldots, \alpha_{n}\right)$ as follows: let $\left\langle w_{0}, b_{0}, w_{1}, b_{1}, \ldots, w_{n-1}, b_{n-1}\right\rangle$ be the

\footnotetext{
${ }^{6}$ Matthew Foreman, contemporary American mathematician

${ }^{7}$ Boban Veličković (b. 1961), Serbian mathematician
} 
partial play in which White follows $a$ (i.e. $\left.w_{k}=a\left(\alpha_{0}, \alpha_{1}, \ldots, \alpha_{k}\right)\right)$ and Black follows $\Sigma$ (i.e. $\left.b_{k}=\Sigma\left(\left\langle w_{0}, b_{0}, w_{1}, b_{1}, \ldots, b_{k-1}, a_{k}\right\rangle\right)\right)$; then we define $a\left(\alpha_{0}, \alpha_{1}, \ldots, \alpha_{n}\right)$ to be an extension of $b_{n-1}$ such that $b\left(\alpha_{0}, \alpha_{1}, \ldots, \alpha_{n}\right)=$ $\max \left(a\left(\alpha_{0}, \alpha_{1}, \ldots, \alpha_{n}\right)\right) \geq \alpha_{n}$.

Now let us prove that the set

$$
C=\left\{\beta<\omega_{1}: b\left(\alpha_{0}, \alpha_{1}, \ldots, \alpha_{n}\right)<\beta \text { whenever } \alpha_{0}<\alpha_{1}<\ldots<\alpha_{n}<\beta\right\}
$$

is closed unbounded. We use standard arguments; first let $\left\langle\beta_{k}: k \in \omega\right\rangle$ be an increasing sequence of elements of $C$, and $\beta=\sup \left\{\beta_{k}: k \in \omega\right\}$. If $\alpha_{0}<\alpha_{1}<\ldots<\alpha_{n}<\beta$, then there is $k \in \omega$ such that $\alpha_{n}<\beta_{k}$ so, since $\beta_{k} \in C$, it follows that $b\left(\alpha_{0}, \alpha_{1}, \ldots, \alpha_{n}\right)<\beta_{k}<\beta$. Thus $C$ is closed. Now suppose $\gamma<\omega_{1}$ and let us find $\beta \in C$ such that $\gamma<\beta$. We define by recursion a sequence $\left\langle\gamma_{k}: k \in \omega\right\rangle$ : let $\gamma_{0}=\gamma$ and for $k \in \omega$ $\gamma_{k+1}=\sup \left\{b\left(\alpha_{0}, \alpha_{1}, \ldots, \alpha_{n}\right): \alpha_{0}<\alpha_{1}<\ldots<\alpha_{n}<\gamma_{k}\right\}$. Finally, let $\beta=\sup \left\{\gamma_{k}: k \in \omega\right\}$. Let us prove that $\beta \in C$ : if $\alpha_{0}<\alpha_{1}<\ldots<\alpha_{n}<\beta$, then there is $k \in \omega$ such that $\alpha_{n}<\gamma_{k}$, so by definition $b\left(\alpha_{0}, \alpha_{1}, \ldots, \alpha_{n}\right)<$ $\gamma_{k+1}<\beta$. Thus $C$ is unbounded as well.

Since $\omega_{1} \backslash A$ is stationary, there is $\beta \in C \cap\left(\omega_{1} \backslash A\right)$. Clearly, all elements of $C$ are limit ordinals, so let $\left\langle\alpha_{n}: n \in \omega\right\rangle$ be an increasing sequence of ordinals such that $\sup \left\{\alpha_{n}: n \in \omega\right\}=\beta$. Let $\left\langle w_{0}, b_{0}, w_{1}, b_{1}, \ldots\right\rangle$ be a play in which White follows $a$ (with respect to ordinals $\alpha_{n}, n<\omega$ ) and Black follows $\Sigma$. Since $\alpha_{n}<\max \left(w_{n}\right)=b\left(\alpha_{0}, \alpha_{1}, \ldots, \alpha_{n}\right)<\beta$ for all $n \in \omega$, we have $\sup \left\{\max \left(w_{n}\right): n \in \omega\right\}=\beta$. This means that there is no closed subset of $A$ containing all ordinals $\max \left(w_{n}\right)$ for $n \in \omega$ (because such set would have to include $\beta$, and $\beta \notin A$ ), so there is no $p \in P_{A}$ such that $p \leq w_{n}$ for all $n \in \omega$. Thus White wins the game, a contradiction.

The proof that White does not have a winning strategy is similar: we begin choosing $\beta \in C \cap A$, and in the end we come up with a closed set $p=\bigcup_{n \in \omega} w_{n} \cup\{\beta\}$ such that $p \leq w_{n}$ for all $n \in \omega$.

The generalization of the game $\mathcal{G}_{\text {inf }}(\omega)$ to uncountable cardinals was defined in [13] by Fuchino ${ }^{8}$, Mildenberger ${ }^{9}$, Shelah and Vojtás. ${ }^{10}$

Definition 2.15 The game $\mathcal{G}_{\text {inf }}(\kappa)$ is played on a partial order $\mathbb{P}$ as follows:

- first, White chooses $a_{0} \in P$;

\footnotetext{
${ }^{8}$ Sakae Fuchino, contemporary Japanese mathematician

${ }^{9}$ Heike Mildenberger, contemporary German mathematician

${ }^{10}$ Peter Vojtáš, contemporary Slovak mathematician
} 
- in his $\alpha$-th move Black chooses $b_{\alpha} \in P$ such that $b_{\alpha} \leq a_{\alpha}$;

- in his $\alpha$-th move (for $\alpha>0$ ) White chooses $a_{\alpha} \in P$ such that $a_{\alpha} \leq b_{\beta}$ for all $\beta<\alpha$.

Theorem $2.16([13])$ White has a winning strategy in $\mathcal{G}_{\text {inf }}(\kappa)$ played on $\mathbb{B}$ iff $\mathbb{B}$ is not $\kappa$-distributive.

\subsection{The cut-and-choose games}

The rest of the games that will be considered here are so-called "cut-andchoose" games. In this section we establish some more notation and prove a few results that will be used in all succeeding sections.

Definition 2.17 Let $\mathbb{B}$ be a Boolean algebra, $p \in B^{+}$, and let $\lambda$ be an infinite cardinal. By $\mathcal{A}_{p}(\lambda)$ we will denote the set of all partitions of $p$ of cardinality $\lambda$, by $\mathcal{A}_{p}(\leq \lambda)$ the set of all partitions of $p$ of cardinality at most $\lambda$, and by $\mathcal{A}_{p}$ the set of all partitions of $p$, regardless of their cardinality.

For every $\lambda \in$ Card and every $A \in \mathcal{A}_{p}(\leq \lambda)$ we fix a sequence $A=\left\{p_{\xi}^{A}\right.$ : $\xi<\lambda\}$ such that, for some $\nu \leq \lambda,\left\{p_{\xi}^{A}: \xi<\nu\right\}$ is an enumeration of $A$, and

$p_{\xi}^{A}=0$ for $\nu \leq \xi<\lambda$. Thus we may get different sequences for the same antichain for various cardinals $\lambda$, but this will not present a problem since $\lambda$ will be a fixed cardinal whenever we use this notation.

Definition 2.18 A cut-and-choose game of type $(\kappa, \lambda, \mu)$, where $\kappa, \lambda \in$ Card, $\kappa$ is infinite and $\mu \in{ }^{\kappa}$ Card, is played on a complete Boolean algebra $\mathbb{B}$ in the following way:

- first, White chooses $p \in B^{+}$. Then, for $\alpha<\kappa$ the players make their moves subsequently:

- in his $\alpha$-th move White chooses a partition $A_{\alpha}$ of $p$ of cardinality at most $\lambda$;

- in his $\alpha$-th move Black chooses a subset $a_{\alpha} \in[\lambda]^{<\mu(\alpha)}$, thus choosing a subset $\left\{p_{\xi}^{A_{\alpha}}: \xi \in a_{\alpha}\right\}$ of the partition $A_{\alpha}$.

We will sometimes abuse this notation in one of the following ways: "a game is of type $(\kappa,<\lambda, \mu)$ " will mean that White chooses partitions of cardinality less than $\lambda$, "a game is of type $(\kappa, \infty, \mu)$ " that the cardinality of 
partitions is not bounded, and "a game is of type $(\kappa, \lambda, \mu)$ ", where $\mu \in$ Card, that Black chooses less than $\mu$ elements in each of his moves.

Hence various cut-and-choose games that will be considered differ in

1) the number $\kappa$ of moves,

2) the number $\lambda$ of elements of each partition,

3) the function $\mu: \kappa \rightarrow$ Card bounding the number of elements that can be chosen by Black in each move and, of course,

4) the winning criterion for White; this condition usually has the form $E\left(\left\langle\bigvee_{\xi \in a_{\alpha}} p_{\xi}^{A_{\alpha}}: \alpha<\kappa\right\rangle\right)=0$ for some function $E:{ }^{\kappa} B \rightarrow B$. Naturally, Black wins iff the condition is not satisfied.

Thus White first picks an element of $B^{+}$. In each of the following $\kappa$ moves he cuts $p$ into at most $\lambda$ pieces and offers them to Black, who then chooses less than $\mu(\alpha)$ of them. This explains the term "cut-and-choose".

For cut-and-choose games we will, as in previous sections, use the notions of a play (now it has the form $\left\langle p, A_{0}, a_{0}, \ldots, A_{\alpha}, a_{\alpha}, \ldots\right\rangle$ ), a partial play, a (winning) strategy etc. So a strategy for White in a cut-and-choose game $\mathcal{G}$ is a pair $\langle p, \Sigma\rangle$ that consists of an element $p \in B^{+}$and a function $\Sigma$ : $\bigcup_{\beta<\kappa} \prod_{\alpha<\beta}\left(\mathcal{A}_{p}(\leq \lambda) \times[\lambda]^{<\mu(\alpha)}\right) \rightarrow \mathcal{A}_{p}(\leq \lambda)$. However, $\Sigma$ essentially depends only on previous moves of the opponent, so there is a way to represent such a function in a simpler way.

Definition 2.19 A function $E:{ }^{\kappa} B \rightarrow B$ is called ascending if for any two sequences $\left\langle a_{\alpha}: \alpha<\kappa\right\rangle,\left\langle b_{\alpha}: \alpha<\kappa\right\rangle \in{ }^{\kappa} B, b_{\alpha} \leq a_{\alpha}$ for all $\alpha<\kappa$ implies $E\left(\left\langle b_{\alpha}: \alpha<\kappa\right\rangle\right) \leq E\left(\left\langle a_{\alpha}: \alpha<\kappa\right\rangle\right)$.

Theorem 2.20 Let $\mathcal{G}$ be a cut-and-choose game of type $(\kappa, \lambda, \mu)$ and let $E:{ }^{\kappa} B \rightarrow B$ be a function such that White wins a play $\left\langle p, A_{0}, a_{0}, \ldots, A_{\alpha}\right.$, $\left.a_{\alpha}, \ldots\right\rangle$ iff $E\left(\left\langle\bigvee_{\xi \in a_{\alpha}} p_{\xi}^{A_{\alpha}}: \alpha<\kappa\right\rangle\right)=0$. For every complete Boolean algebra $\mathbb{B}$ the following conditions are equivalent:

(a) White has a winning strategy in the game $\mathcal{G}$ played on $\mathbb{B}$;

(b) there are $p \in B^{+}$and $w: \bigcup_{\beta<\kappa} \prod_{\alpha<\beta}[\lambda]^{<\mu(\alpha)} \rightarrow \mathcal{A}_{p}(\leq \lambda)$ such that

$$
\forall i \in \prod_{\alpha<\kappa}[\lambda]^{<\mu(\alpha)} E\left(\left\langle\bigvee_{\xi \in i(\alpha)} p_{\xi}^{w(i \uparrow \alpha)}: \alpha<\kappa\right\rangle\right)=0
$$


Furthermore, if the function $E$ is ascending and $\mathbb{B}$ satisfies

$$
\forall p \in B^{+} \text {c.c. }(\langle p \downarrow, \leq\rangle)>\lambda \text {, }
$$

then these conditions are also equivalent to

(c) there are $p \in B^{+}$and $w: \bigcup_{\beta<\kappa} \prod_{\alpha<\beta}[\lambda]^{<\mu(\alpha)} \rightarrow \mathcal{A}_{p}(\lambda)$ such that (2.1) holds.

Note. 1) From the proof below it will be clear that (c) $\Rightarrow(a) \Leftrightarrow(b)$ holds for every complete Boolean algebra $\mathbb{B}$; the additional conditions are needed only to prove $(b) \Rightarrow(c)$. 2) Clearly, in the games in which there is no bound on the cardinality of partitions chosen by White, $\lambda$ can be substituted by c.c. $(\mathbb{B})$ in the formulation of the previous theorem.

Proof. $(\mathrm{a}) \Rightarrow(\mathrm{b})$. Let $p$ and $\Sigma$ make a winning strategy for White in the game $\mathcal{G}$. We define $w$ by recursion on $|w|$. First, let $w(\emptyset)=\Sigma(\emptyset)$. If $\alpha<\kappa$, $\varphi \in \prod_{\beta<\alpha}[\lambda]^{<\mu(\beta)}$, and $w(\varphi\lceil\beta)$ is already defined for $\beta<\alpha$, let us define

$$
w(\varphi)=\Sigma(\langle\langle w(\varphi \mid \beta), \varphi(\beta)\rangle: \beta<\alpha\rangle) .
$$

Let $i \in \prod_{\alpha<\kappa}[\lambda]^{<\mu(\alpha)}$; we prove $(2.1)$.

Claim. In the match in which White begins with $p$ and Black plays $\langle i(\alpha): \alpha<\kappa\rangle$, White, following $\Sigma$, plays $A_{\alpha}=w(i\lceil\alpha)$ in the $\alpha$-th move.

Proof. Clearly, the first move of White is $A_{0}=\Sigma(\emptyset)=w(\emptyset)$. Suppose Claim holds for $\beta<\alpha$. Then White, in his $\alpha$-th move, plays $\Sigma(\langle\langle w(i \mid$ $\beta), i(\beta)\rangle: \beta<\alpha\rangle)=w(i\lceil\alpha)$.

Since White wins the play $\left\langle p, A_{0}, i(0), \ldots, A_{\alpha}, i(\alpha), \ldots\right\rangle$, we have that $E\left(\left\langle\bigvee_{\xi \in i(\alpha)} p_{\xi}^{A_{\alpha}}: \alpha<\kappa\right\rangle\right)=0$ and $(2.1)$ is proved.

(b) $\Rightarrow(\mathrm{c})$. Let $p, w$ be as in (b). We define $w^{\prime}(\varphi) \in \mathcal{A}_{p}(\lambda)$ and $\psi(\varphi) \in$ $\bigcup_{\beta<\kappa} \prod_{\alpha<\beta}[\lambda]^{<\mu(\alpha)}$ by recursion on length of $\varphi \in \bigcup_{\beta<\kappa} \prod_{\alpha<\beta}[\lambda]^{<\mu(\alpha)}$, so that

(i) $\operatorname{dom} \psi(\varphi)=\operatorname{dom} \varphi$;

(ii) if $\alpha \in \operatorname{dom}(\varphi)$, then $\psi(\varphi\lceil\alpha)=\psi(\varphi) \uparrow \alpha$;

(iii) $w^{\prime}(\varphi)$ is a refinement of $w(\psi(\varphi))$.

Let $\beta<\kappa$ and $\varphi \in \prod_{\alpha<\beta}[\lambda]^{<\mu(\alpha)}$, and suppose $\psi\left(\varphi\lceil\gamma)\right.$ and $w^{\prime}(\varphi\lceil\gamma)$ are already defined for $\gamma<\beta$ so that (i)-(iii) hold. If $\beta=\gamma+1$, we put $C=\left\{\xi<\lambda: \exists \zeta \in \varphi(\gamma) p_{\zeta}^{w^{\prime}(\varphi \mid \gamma)} \leq p_{\xi}^{w(\psi(\varphi)\lceil\gamma)}\right\}$ and $\psi(\varphi)=\psi\left(\varphi\lceil\gamma)^{\frown} C\right.$. Since $|\varphi(\gamma)|<\mu(\gamma)$, it follows that $|\psi(\varphi)(\gamma)|=|C|<\mu(\gamma)$ as well. If $\beta$ is 
a limit ordinal, let $\psi(\varphi)=\bigcup_{\alpha<\beta} \psi(\varphi \mid \alpha)$. Clearly, (i) and (ii) hold. Now let $\left\{b_{\zeta}: \zeta<\lambda\right\}$ be a maximal antichain in $p \downarrow$ of cardinality $\lambda$ (existing by $(2.2))$ and let $w^{\prime}(\varphi)=\left\{b_{\zeta} \wedge x: \zeta<\lambda, x \in w(\psi(\varphi))\right\} \backslash\{0\}$. Obviously, $w^{\prime}(\varphi)$ is a refinement of $w(\psi(\varphi))$ of cardinality $\lambda$, so (iii) holds as well.

Let $i \in \prod_{\alpha<\kappa}[\lambda]^{<\mu(\alpha)}$ be arbitrary and let us prove

$$
E\left(\left\langle\bigvee_{\zeta \in i(\alpha)} p_{\zeta}^{w^{\prime}(i\lceil\alpha)}: \alpha<\kappa\right\rangle\right)=0
$$

We define $j=\bigcup_{\alpha<\kappa} \psi\left(i\lceil\alpha)\right.$. By (i) and (ii) we have $j \in \prod_{\alpha<\kappa}[\lambda]^{<\mu(\alpha)}$. Condition (b) implies that

$$
E\left(\left\langle\bigvee_{\xi \in j(\alpha)} p_{\xi}^{w(j\lceil\alpha)}: \alpha<\kappa\right\rangle\right)=0
$$

Now, by (iii), for every $\alpha<\kappa$ and every $\zeta \in i(\alpha)$ there is $\xi \in j(\alpha)$ such that $p_{\zeta}^{w^{\prime}(i\lceil\alpha)} \leq p_{\xi}^{w(j\lceil\alpha)}$, so we have

$$
\bigvee_{\zeta \in i(\alpha)} p_{\zeta}^{w^{\prime}(i\lceil\alpha)} \leq \bigvee_{\xi \in j(\alpha)} p_{\xi}^{w(j\lceil\alpha)} .
$$

Now, the fact that $E$ is ascending and (2.4) imply (2.3), which proves (c).

(c) $\Rightarrow($ a). Let $p$ and $w$ be the objects given by (c); the strategy for White will consist of $p$ and the function $\Sigma$ defined by recursion as follows. First let $\Sigma(\emptyset)=w_{\emptyset}$. In the $\alpha$-th step, let $\Sigma\left(\left\langle\left\langle A_{\beta}, a_{\beta}\right\rangle: \beta<\alpha\right\rangle\right)=w\left(\left\langle a_{\beta}: \beta<\alpha\right\rangle\right)$.

We prove that $\langle p, \Sigma\rangle$ is a winning strategy for White. Let $\left\langle p, A_{0}, a_{0}, \ldots\right.$, $\left.A_{\alpha}, a_{\alpha}, \ldots\right\rangle$ be an arbitrary play in which White follows this strategy. Since $\left\langle a_{\alpha}: \alpha<\kappa\right\rangle \in \prod_{\alpha<\kappa}[\lambda]^{<\mu(\alpha)},(2.1)$ guarantees that $E\left(\left\langle\bigvee_{\xi \in a_{\alpha}} p_{\xi}^{w(a \mid \alpha)}: \alpha<\right.\right.$ $\kappa\rangle)=0$. But in the $\alpha$-th move White plays $A_{\alpha}=w(a \uparrow \alpha)$, so we have $E\left(\left\langle\bigvee_{\xi \in a_{\alpha}} p_{\xi}^{A_{\alpha}}: \alpha<\kappa\right\rangle\right)=0$ and White wins the game.

The case $\lambda=\mu=2$ will appear in many games, and when it does we can simplify the moves of both players in the following way: instead of a partition $A_{\alpha}=\left\{p_{0}^{A_{\alpha}}, p_{1}^{A_{\alpha}}\right\}$ of $p$, in each of his moves White can choose only $p_{\alpha}=p_{0}^{A_{\alpha}}$, and $p_{1}^{A_{\alpha}}=p \wedge p_{\alpha}^{\prime}$ is then uniquely determined. Also, Black chooses one of those two elements, so if we introduce the following notation:

$$
p_{\alpha}^{k}= \begin{cases}p_{\alpha} & \text { if } k=0 \\ p \wedge p_{\alpha}^{\prime} & \text { if } k=1\end{cases}
$$

we may assume that Black, instead of $p_{\alpha}^{i_{\alpha}}$, only chooses $i_{\alpha} \in\{0,1\}$, so in this case we can also call the sequence $\left\langle p, p_{0}, i_{0}, \ldots, p_{\alpha}, i_{\alpha}, \ldots\right\rangle$ a play. The previous theorem now takes the following form: 
Theorem 2.21 Let $\mathcal{G}$ be a cut-and-choose game of type $(\kappa, 2,2)$, and let $E$ : ${ }^{\kappa} B \rightarrow B$ be a function such that White wins a play $\left\langle p, p_{0}, i_{0}, \ldots, p_{\alpha}, i_{\alpha}, \ldots\right\rangle$ iff $E\left(\left\langle p_{\alpha}^{i_{\alpha}}: \alpha<\kappa\right\rangle\right)=0$. For every complete Boolean algebra $\mathbb{B}$ the following conditions are equivalent:

(a) White has a winning strategy in the game $\mathcal{G}$ on $\mathbb{B}$;

(b) there are $p \in B^{+}$and $w:{ }^{<\kappa} 2 \rightarrow[0, p]_{\mathbb{B}}$ such that

$$
\forall i \in{ }^{\kappa} 2 E\left(\left\langle w_{i \uparrow \alpha}^{i(\alpha)}: \alpha<\kappa\right\rangle\right)=0 .
$$

If, in addition, $E$ is ascending, then these conditions are also equivalent to

(c) there are $p \in B^{+}$and $w:{ }^{<\kappa} 2 \rightarrow(0, p)_{\mathbb{B}}$ such that $(2.5)$ holds.

(The proof of $(\mathrm{b}) \Rightarrow(\mathrm{c})$ must be slightly modified: in $\mathcal{A}_{p}(\leq 2) \backslash \mathcal{A}_{p}(2)$ we have only the trivial antichain $\{p\}$; thus in the definition of $w^{\prime}(\varphi)$ we take $w^{\prime}(\varphi)=w(\psi(\varphi))$ if $w(\psi(\varphi)) \neq\{p\}$, and otherwise we take $w^{\prime}(\varphi)$ to be any partition of $p$ into two elements.)

A strategy for Black is $\left\langle\Sigma_{p}: p \in B^{+}\right\rangle$, where

$$
\Sigma_{p}: \bigcup_{\beta<\kappa}\left(\prod_{\alpha<\beta}\left(\mathcal{A}_{p}(\leq \lambda) \times[\lambda]^{<\mu(\alpha)}\right) \times \mathcal{A}_{p}(\leq \lambda)\right) \rightarrow[\lambda]^{<\mu(\beta)}
$$

for each $p \in B^{+}$. Again, we can considerably simplify the condition of existence of a winning strategy. Thus, the statement analogous to Theorem 2.20 for Black is:

Theorem 2.22 Let $\mathcal{G}$ be a cut-and-choose game of type $(\kappa, \lambda, \mu)$, and let $E:{ }^{\kappa} B \rightarrow B$ be a function such that White wins a play $\left\langle p, A_{0}, a_{0}, \ldots, A_{\alpha}\right.$, $\left.a_{\alpha}, \ldots\right\rangle$ iff $E\left(\left\langle\bigvee_{\xi \in a_{\alpha}} p_{\xi}^{A_{\alpha}}: \alpha<\kappa\right\rangle\right)=0$. For every complete Boolean algebra $\mathbb{B}$ the following conditions are equivalent:

(a) Black has a winning strategy in the game $\mathcal{G}$ on $\mathbb{B}$;

(b) for every $p \in B^{+}$there is $w: \bigcup_{\beta<\kappa}{ }^{\beta+1} \mathcal{A}_{p}(\leq \lambda) \rightarrow[\lambda]^{<\mu(\beta)}$ such that

$$
\forall i \in{ }^{\kappa} \mathcal{A}_{p}(\leq \lambda) E\left(\left\langle\bigvee_{\xi \in w(i \uparrow(\alpha+1))} p_{\xi}^{i(\alpha)}: \alpha<\kappa\right\rangle\right)>0
$$

Furthermore, if $E$ is ascending and $\mathbb{B}$ satisfies (2.2), then these conditions are also equivalent to 
(c) for every $p \in B^{+}$there is $w: \bigcup_{\beta<\kappa}{ }^{\beta+1} \mathcal{A}_{p}(\lambda) \rightarrow[\lambda]^{<\mu(\beta)}$ such that

$$
\forall i \in{ }^{\kappa} \mathcal{A}_{p}(\lambda) E\left(\left\langle\bigvee_{\xi \in w(i\lceil(\alpha+1))} p_{\xi}^{i(\alpha)}: \alpha<\kappa\right\rangle\right)>0
$$

Note. As in Theorem 2.20, only one implication requires the additional assumption that $E$ is ascending; (a) $\Leftrightarrow(\mathrm{b}) \Rightarrow(\mathrm{c})$ holds for every complete Boolean algebra $\mathbb{B}$.

Proof. $(\mathrm{a}) \Rightarrow(\mathrm{c})$ Suppose Black has a winning strategy $\left\langle\Sigma_{p}: p \in B^{+}\right\rangle$in $\mathcal{G}$, and let $p \in B^{+}$. We define $w$ by recursion. If $\alpha<\kappa, \varphi \in{ }^{\alpha+1} \mathcal{A}_{p}(\lambda)$, and $w(\varphi \uparrow(\gamma+1))$ is already defined for each $\gamma<\alpha$, let us define

$$
w(\varphi)=\Sigma_{p}(\langle\langle\langle\varphi(\gamma), w(\varphi \uparrow(\gamma+1))\rangle: \gamma<\alpha\rangle, \varphi(\alpha)\rangle) .
$$

Let $i \in{ }^{\kappa} \mathcal{A}_{p}(\lambda)$; we prove (2.7). An easy induction shows that, in the match in which White begins with $p$ and then plays $\langle i(\alpha): \alpha<\kappa\rangle$, in the $\alpha$-th move Black, following $\Sigma_{p}$, plays $a_{\alpha}=w(i \uparrow(\alpha+1))$. Since Black wins, we have $E\left(\left\langle\bigvee_{\xi \in a_{\alpha}} p_{\xi}^{i(\alpha)}: \alpha<\kappa\right\rangle\right)=0$ and (2.7) is proved.

(c) $\Rightarrow$ (b) Let $p \in B^{+}$and let $w$ be the function provided by (c). By recursion on $\varphi \in \bigcup_{\beta<\kappa}{ }^{\beta+1} \mathcal{A}_{p}(\leq \lambda)$, for $\operatorname{dom} \varphi=\beta+1$, we define $w^{\prime}(\varphi) \in$ $[\lambda]^{<\mu(\beta)}$ and $\psi(\varphi) \in{ }^{\beta+1} \mathcal{A}_{p}(\lambda)$ such that

(i) if $\alpha<\beta$, then $\psi(\varphi \uparrow(\alpha+1))=\psi(\varphi) \uparrow(\alpha+1)$;

(ii) $\psi(\varphi)(\beta)$ is a refinement of $\varphi(\beta)$;

(iii) $\bigvee_{\zeta \in w(\psi(\varphi))} p_{\zeta}^{\psi(\varphi)(\beta)} \leq \bigvee_{\xi \in w^{\prime}(\varphi)} p_{\xi}^{\varphi(\beta)}$

Let $\varphi \in{ }^{\beta+1} \mathcal{A}_{p}(\leq \lambda)$ and suppose $\psi(\varphi \uparrow(\gamma+1))$ and $w^{\prime}(\varphi \uparrow(\gamma+1))$ are already defined for $\gamma<\beta$ so that (i)-(iii) hold. Let $\left\{b_{\zeta}: \zeta<\lambda\right\}$ be a partition of $p$ of cardinality $\lambda$ (existing by (2.2)) and let $C=\left\{b_{\zeta} \wedge x: \zeta<\lambda, x \in\right.$ $\varphi(\beta)\} \backslash\{0\}$. Now we define $\psi(\varphi)=\bigcup_{\gamma<\beta} \psi(\varphi \uparrow(\gamma+1))^{\wedge} C$. Obviously, (i) and (ii) hold. Next we put $w^{\prime}(\varphi)=\left\{\xi<\lambda: \exists \zeta \in w(\psi(\varphi)) p_{\zeta}^{\psi(\varphi)(\beta)} \leq p_{\xi}^{\varphi(\beta)}\right\}$. Taking (ii) into account, it is easy to see that (iii) also holds.

Let $i \in{ }^{\kappa} \mathcal{A}_{p}(\leq \lambda)$ be arbitrary and let us prove

$$
E\left(\left\langle\bigvee_{\xi \in w^{\prime}(i \uparrow(\alpha+1))} p_{\xi}^{i(\alpha)}: \alpha<\kappa\right\rangle\right)>0
$$

We define $j=\bigcup_{\alpha<\kappa} \psi(i \uparrow(\alpha+1))$. By (i) we have $j \in{ }^{\kappa} \mathcal{A}_{p}(\lambda)$. The condition (c) implies that

$$
E\left(\left\langle\bigvee_{\zeta \in w(j \uparrow(\alpha+1))} p_{\zeta}^{j(\alpha)}: \alpha<\kappa\right\rangle\right)>0
$$


Now, by (iii) and the fact that $E$ is ascending we have (2.5), which proves (b).

(b) $\Rightarrow$ (a) For $p \in B^{+}$we define by recursion the functions $\Sigma_{p}$ that make a strategy for Black. Let $p \in B^{+}$and let $w$ be given by (b); in the $\alpha$-th step, let $\Sigma_{p}\left(\left\langle\left\langle\left\langle A_{\beta}, a_{\beta}\right\rangle: \beta<\alpha\right\rangle, A_{\alpha}\right\rangle\right)=w\left(\left\langle A_{\beta}: \beta \leq \alpha\right\rangle\right)$.

We prove that $\left\langle\Sigma_{p}: p \in B^{+}\right\rangle$is a winning strategy. Let $\left\langle p, A_{0}, a_{0}, \ldots\right.$, $\left.A_{\alpha}, a_{\alpha}, \ldots\right\rangle$ be an arbitrary play in which Black follows this strategy. Since $\left\langle A_{\alpha}: \alpha<\kappa\right\rangle \in{ }^{\kappa} \mathcal{A}_{p}(\leq \lambda),(2.6)$ guarantees that $E\left(\left\langle\bigvee_{\xi \in w(A \uparrow(\alpha+1))} p_{\xi}^{A_{\alpha}}: \alpha<\right.\right.$ $\kappa\rangle)>0$. But in the $\alpha$-th move Black plays $a_{\alpha}=w(A \uparrow(\alpha+1))$, so we have $E\left(\left\langle\bigvee_{\xi \in a_{\alpha}} p_{\xi}^{A_{\alpha}}: \alpha<\kappa\right\rangle\right)>0$ and Black wins the game.

For games of type $(\kappa, 2,2)$ the theorem above takes the following form:

Theorem 2.23 Let $\mathcal{G}$ be a cut-and-choose game of type $(\kappa, 2,2)$ and let $E$ : ${ }^{\kappa} B \rightarrow B$ be a function such that White wins a play $\left\langle p, p_{0}, i_{0}, \ldots, p_{\alpha}, i_{\alpha}, \ldots\right\rangle$ iff $E\left(\left\langle p_{\alpha}^{i_{\alpha}}: \alpha<\kappa\right\rangle\right)=0$. For every complete Boolean algebra $\mathbb{B}$ the following conditions are equivalent:

(a) Black has a winning strategy in the game $\mathcal{G}$ on $\mathbb{B}$;

(b) for every $p \in B^{+}$there is $w: \bigcup_{\beta<\kappa}{ }^{\beta+1}(0, p)_{\mathbb{B}} \rightarrow 2$ such that for every $i \in{ }^{\kappa}(0, p)_{\mathbb{B}}$

$$
E\left(\left\langle i(\alpha)^{w(i \uparrow(\alpha+1))}: \alpha<\kappa\right\rangle\right)>0 .
$$

Furthermore, if $E$ is ascending, then these conditions are also equivalent to

(c) for every $p \in B^{+}$there is $w: \bigcup_{\beta<\kappa}{ }^{\beta+1}[0, p]_{\mathbb{B}} \rightarrow 2$ such that for every $i \in{ }^{\kappa}[0, p]_{\mathbb{B}}(2.10)$ holds.

\subsection{The game $\mathcal{G}_{\text {dist }}(\kappa, \lambda, \mu)$}

The investigation of cut-and-choose games began in 1984 when Jech in [16] introduced the game $\mathcal{G}_{\text {dist }}(\omega, 2,2)$. (The notation we use differs from his.) Again, unless we state otherwise, all results are due to Jech.

Definition 2.24 Let $\kappa$ be an infinite cardinal, and let $\lambda, \mu$ be cardinals. The distributivity game $\mathcal{G}_{\text {dist }}(\kappa, \lambda, \mu)$ is a cut-and-choose game of type $(\kappa, \lambda, \mu)$ : it is played in $\kappa$-many steps; after choosing an element $p \in B^{+}$White chooses, in his every move, a partition $A_{\alpha}$ of cardinality at most $\lambda$, and Black chooses a set $a_{\alpha} \in[\lambda]^{<\mu}$. White wins iff $\bigwedge_{\alpha<\kappa} \bigvee_{\xi \in a_{\alpha}} p_{\xi}^{A_{\alpha}}=0$, and Black wins otherwise. 
Theorem 2.25 Let $\mathbb{B}$ be a complete Boolean algebra and $\lambda$ an infinite cardinal. White has a winning strategy in the game $\mathcal{G}_{\text {dist }}(\omega, \lambda, 2)$ iff $\mathbb{B}$ is not $(\omega, \lambda)$-distributive.

Proof. $(\Leftarrow)$ Suppose that $\mathbb{B}$ is not $(\omega, \lambda)$-distributive. By Theorem 1.59 there are $a \in B$ and partitions $W_{n}$ (for $n \in \omega$ ) of $a$ such that $\left|W_{n}\right| \leq \lambda$, and if $b_{n} \in W_{n}$ for every $n \in \omega$, then $\bigwedge b_{n}=0$. A winning strategy for White is to play $W_{n}$ in his $n$-th move.

$(\Rightarrow)$ Conversely, if White has a winning strategy, Theorem 2.20 claims that there are $a \in B$ and $w:{ }^{<\omega} \lambda \rightarrow \mathcal{A}_{a}^{\prime}(\lambda)$ such that

$$
\forall i \in{ }^{\omega} \lambda \bigwedge_{n<\omega} p_{i(n)}^{w(i \uparrow n)}=0 .
$$

For all $n \in \omega$ and all $s \in{ }^{n} 2$, let us define $Q_{s}=\left\{\bigwedge_{k \leq n} b_{k}: b_{k} \in w(s \uparrow\right.$ $k$ ) for all $k \leq n\} \backslash\{0\}$ and $P_{n}=\bigcup_{s \in n_{2}} Q_{s}$. Clearly, since $w\left(s\lceil k) \in \mathcal{A}_{a}^{\prime}(\lambda)\right.$ for all $s \in{ }^{n} 2$ and all $k<n$, each $P_{n}$ is an antichain below $a$ of cardinality at most $\lambda$. It is easy to prove by induction that $P_{n}$ is a partition of $a$ and that $P_{n+1}$ is a refinement of $P_{n}$ for $n \in \omega$. Let $p_{n} \in P_{n}(n<\omega)$ be such that $p_{n+1} \leq p_{n}$ for all $n$. For each $n$ there are $s_{n} \in{ }^{n} 2$ such that $p_{n} \in Q_{s_{n}}$, and $b_{n} \in w\left(s_{n}\right)$ such that $p_{n}=p_{n-1} \wedge b_{n}$. From the definition of $Q_{s}$ it follows that $s_{n}=s_{n-1} \curvearrowright \alpha$, where $b_{n}$ is the $\alpha$-th element in the enumeration of $w\left(s_{n-1}\right)$ (otherwise, if $s_{n}(k) \neq s_{n-1}(k)$ for some $k<n$, the elements $p_{n}$ and $p_{n-1}$ would be incompatible). Let $i=\bigcup_{n<\omega} s_{n}$. Then $\bigwedge_{n \in \omega} p_{n} \leq \bigwedge_{n<\omega} b_{n}=\bigwedge_{n<\omega} p_{s_{n+1}(n)}^{w\left(s_{n}\right)}=\bigwedge_{n<\omega} p_{i(n)}^{w(i \uparrow n)}=0$, so by Theorem $1.59 \mathbb{B}$ is not $(\omega, \lambda)$-distributive.

There are many variations of this game. In the first of them, the game $\mathcal{G}_{\text {dist }}(\omega, \infty, 2)$, White is allowed to make partitions of any size. The proof of the following theorem is analogous to the proof of Theorem 2.25.

Theorem 2.26 Let $\mathbb{B}$ be a complete Boolean algebra. White has a winning strategy in the game $\mathcal{G}_{\text {dist }}(\omega, \infty, 2)$ iff $\mathbb{B}$ is not $\omega$-distributive.

Thus White has a winning strategy in the game $\mathcal{G}_{\text {dist }}(\omega, \infty, 2)$ iff he has one in $\mathcal{G}_{\text {inf }}(\omega)$. The same holds for Black (the first implication was proved by Jech, and the second by Veličković):

Theorem 2.27 Black has a winning strategy in $\mathcal{G}_{\text {inf }}(\omega)$ played on a complete Boolean algebra $\mathbb{B}$ iff he has a winning strategy in $\mathcal{G}_{\text {dist }}(\omega, \infty, 2)$ played on $\mathbb{B}$. 
Proof. $(\Rightarrow)$ Let $\Sigma: \bigcup_{n<\omega} D\left({ }^{2 n+1} B\right) \rightarrow B$ be a winning strategy for Black in $\mathcal{G}_{\text {inf }}(\omega)$, and let $a \in B^{+}$be the first move of White in $\mathcal{G}_{\text {dist }}(\omega, \infty, 2)$. We denote $\nu=$ c.c. $(\mathbb{B})$ (a bound on the cardinality of partitions of $a$ ) and define a function $w: \bigcup_{n<\omega}{ }^{n+1} \mathcal{A}_{a}^{\prime}(\nu) \rightarrow \nu$ such that

$$
\forall i \in{ }^{\omega} \mathcal{A}_{a}^{\prime}(\nu) \bigwedge_{n<\omega} p_{w(i \uparrow(n+1))}^{i(n)}>0 .
$$

By Theorem 2.22 (applied for $\lambda=\nu$ ) it will follow that Black has a winning strategy in $\mathcal{G}_{\text {dist }}(\omega, \infty, 2)$.

We proceed by recursion. Assume that $w$ has been defined for all sequences of length at most $n$ of partitions of $a$, and let $\left\langle W_{0}, \ldots, W_{n}\right\rangle \in$ ${ }^{n+1} \mathcal{A}_{a}^{\prime}(\nu)$. Let us define elements $a_{k}, b_{k}, c_{k}$ for $k \leq n$ as follows: first let $a_{0}=a$. For $0 \leq k \leq n$ let $b_{k}=\Sigma\left(\left\langle a_{0}, b_{0}, \ldots, b_{k-1}, a_{k}\right\rangle\right)$. There is $c_{k} \in W_{k}$ such that $b_{k} \wedge c_{k} \neq 0$; let $a_{k+1}=b_{k} \wedge c_{k}$. Finally, let $w\left(\left\langle W_{0}, \ldots, W_{n}\right\rangle\right)=\alpha$, where $c_{n}=p_{\alpha}^{W_{n}}$, i.e. $c_{n}$ is the $\alpha$-th element in the enumeration of $W_{n}$.

Let us prove that (2.11) holds. Let $i=\left\langle W_{n}: n \in \omega\right\rangle \in{ }^{\omega} \mathcal{A}_{a}^{\prime}(\nu)$. If we construct $a_{n}, b_{n}, c_{n}$ for $n \in \omega$ as above, it is easy to prove by induction that $p_{w(i \uparrow(n+1))}^{i(n)}=c_{n}$ for all $n$. Furthermore, $c_{n} \geq a_{n+1} \geq b_{n+1}$ holds, and $\left\langle a_{0}, b_{0}, \ldots, a_{n}, b_{n}, \ldots\right\rangle$ is a play in the game $\mathcal{G}_{\text {inf }}(\omega)$ in which Black follows $\Sigma$, so we have $\bigwedge_{n<\omega} b_{n}>0$. It follows that $\bigwedge_{n \in \omega} c_{n}>0$ as well, which completes the proof.

$(\Leftarrow)$ Let $a \in B^{+}$be the first move of White in $\mathcal{G}_{\text {inf }}(\omega)$. If we assume that Black has a winning strategy in $\mathcal{G}_{\text {dist }}(\omega, \infty, 2)$, Theorem 2.22 implies the existence of a function $w: \bigcup_{n<\omega}{ }^{n+1} \mathcal{A}_{a}^{\prime}(\nu) \rightarrow \nu$ such that $(2.11)$ holds. We define a strategy $\Sigma: \bigcup_{n<\omega} D\left({ }^{2 n+1} B\right) \rightarrow B$ for Black in $\mathcal{G}_{\text {inf }}(\omega)$ by recursion.

Assume that, for some $n<\omega, \Sigma$ has been defined for all sequences of length $2 k+1(k<n)$ of elements of $B$, and a sequence $\left\langle a_{0}, b_{0}, \ldots, a_{n}\right\rangle$ is given $\left(a_{0}=a\right)$. We define partitions $A_{k}(k<n)$ as follows. First, let $S_{0}=\left\{p_{w(\langle A\rangle)}^{A}: A \in \mathcal{A}_{a}^{\prime}(\nu)\right\}$. If $S_{0}$ contains all nonzero elements below $b_{0}$ (including $b_{0}$ itself), we let $A_{0}$ be a partition of $a$ such that $p_{w\left(\left\langle A_{0}\right\rangle\right)}^{A_{0}}=a_{1}$, otherwise let $A_{0}$ be arbitrary. In the $k$-th step, we let $S_{k}=\left\{p_{w\left(\left\langle A_{0}, A_{1}, \ldots, A_{k-1}, A\right\rangle\right)}^{A}: A \in \mathcal{A}_{a}^{\prime}(\nu)\right\}$; if for all $l \leq k S_{l}$ contains all nonzero elements below $b_{l}$, we let $A_{k}$ be a partition of $a$ such that $p_{w\left(\left\langle A_{0}, A_{1}, \ldots, A_{k}\right\rangle\right)}^{A_{k}}=a_{k+1}$, otherwise let $A_{k}$ be arbitrary. Finally, we claim that there is nonzero $b_{n} \leq a_{n}$ such that $S_{n}$ contains all nonzero elements below $b_{n}$. Suppose not; then the set $a_{n} \downarrow \backslash S_{n}$ is dense below $a_{n}$. If $A$ is a maximal antichain of elements in $a_{n} \downarrow \backslash S_{n}$, then $A$ is a partition of $a_{n}$ (otherwise there would be $d \leq\left(a_{n} \downarrow \backslash \bigvee A\right)$ that is not in $S_{n}$, a contradiction with the maximality of $A$ ), and we would have $p_{w\left(\left\langle A_{0}, A_{1}, \ldots, A_{n-1}, A\right\rangle\right)}^{A} \in A \cap S_{n}$, 
which is impossible. We let $\Sigma\left(\left\langle a_{0}, b_{0}, \ldots, a_{n}\right\rangle\right)=b_{n}$.

Let us prove that $\Sigma$ is a winning strategy. Let $\left\langle a_{0}, b_{0}, \ldots, a_{n}, b_{n}, \ldots\right\rangle$ be a play in which Black follows $\Sigma$. If we construct $A_{n}$ for $n \in \omega$ as above and define $i \in{ }^{\omega} \mathcal{A}_{a}^{\prime}(\nu)$ by $i(n)=A_{n}$, we have $p_{w(i \uparrow(n+1))}^{i(n)}=a_{n+1}$ for all $n<\omega$, so by $(2.11) \bigwedge_{n<\omega} a_{n}>0$. Hence Black wins $\mathcal{G}_{\text {inf }}(\omega)$.

It is now clear that the existence of a winning strategy for Black in $\mathcal{G}_{\text {inf }}(\omega)$ implies the existence of such strategy in $\mathcal{G}_{\text {dist }}(\omega, 2,2)$. Some results concerning the reverse implication were obtained by Zapletal ${ }^{11}$ :

Theorem 2.28 ([38]) If Black has a winning strategy in $\mathcal{G}_{\text {dist }}(\omega, 2,2)$ played on a complete Boolean algebra $\mathbb{B}$ and $\mathbb{B}$ satisfies the $\mathfrak{c}^{+}-$c.c., then he also has a winning strategy in $\mathcal{G}_{\text {inf }}(\omega)$ played on $\mathbb{B}$.

Theorem 2.29 ([38]) If $0^{\sharp}$ does not exist, and Black has a winning strategy in $\mathcal{G}_{\text {dist }}(\omega, 2,2)$ played on a complete Boolean algebra $\mathbb{B}$, then he also has a winning strategy in $\mathcal{G}_{\text {inf }}(\omega)$ played on $\mathbb{B}$.

An example was provided (assuming there is a measurable cardinal) in [38] showing that, in general, the existence of a winning strategy for Black in $\mathcal{G}_{\text {dist }}(\omega, 2,2)$ does not imply the existence of a winning strategy for Black in $\mathcal{G}_{\text {inf }}(\omega)$.

We now turn to the generalized game $\mathcal{G}_{\text {dist }}(\kappa, \lambda, \mu)$. We begin with noting some obvious comparisons.

Lemma 2.30 (a) Let $\kappa<\kappa_{1}$. If White has a winning strategy in $\mathcal{G}_{\text {dist }}(\kappa$, $\lambda, \mu)$, then he has a winning strategy in $\mathcal{G}_{\text {dist }}\left(\kappa_{1}, \lambda, \mu\right)$. If Black has a winning strategy in $\mathcal{G}_{\text {dist }}\left(\kappa_{1}, \lambda, \mu\right)$, then he has a winning strategy in $\mathcal{G}_{\text {dist }}(\kappa, \lambda, \mu)$.

(b) Let $\lambda<\lambda_{1}$. If White has a winning strategy in $\mathcal{G}_{\text {dist }}(\kappa, \lambda, \mu)$, then he has a winning strategy in $\mathcal{G}_{\text {dist }}\left(\kappa, \lambda_{1}, \mu\right)$. If Black has a winning strategy in $\mathcal{G}_{\text {dist }}\left(\kappa, \lambda_{1}, \mu\right)$, then he has a winning strategy in $\mathcal{G}_{\text {dist }}(\kappa, \lambda, \mu)$.

(c) Let $\mu<\mu_{1}$. If White has a winning strategy in $\mathcal{G}_{\text {dist }}\left(\kappa, \lambda, \mu_{1}\right)$, then he has a winning strategy in $\mathcal{G}_{\text {dist }}(\kappa, \lambda, \mu)$. If Black has a winning strategy in $\mathcal{G}_{\text {dist }}(\kappa, \lambda, \mu)$, then he has a winning strategy in $\mathcal{G}_{\text {dist }}\left(\kappa, \lambda, \mu_{1}\right)$.

\footnotetext{
${ }^{11}$ Jindřih Zapletal, contemporary Czech matematician
} 
One direction of Theorem 2.25 can be obtained for any $\kappa, \lambda$ by modifying the proof:

Theorem 2.31 ([9], Theorem 1.3) If White does not have a winning strategy in the game $\mathcal{G}_{\text {dist }}(\kappa, \lambda, 2)$ played on a complete Boolean algebra $\mathbb{B}$, then $\mathbb{B}$ is $(\kappa, \lambda)$-distributive.

Dobrinen $^{12}$ also obtained a partial converse:

Theorem 2.32 ([9], Theorem 1.4) If White has a winning strategy in the game $\mathcal{G}_{\text {dist }}(\kappa, \lambda, 2)$ played on a complete Boolean algebra $\mathbb{B}$, then

(a) $\mathbb{B}$ is not $\left(\lambda^{<\kappa}, \lambda\right)$-distributive;

(b) $\mathbb{B}$ is not $\left(\kappa, \lambda^{<\kappa}\right)$-distributive.

Corollary 2.33 White has a winning strategy in the game $\mathcal{G}_{\text {dist }}(\kappa, 2,2)$ played on a complete Boolean algebra $\mathbb{B}$ iff $\mathbb{B}$ is not $(\kappa, 2)$-distributive.

Proof. $(\Leftarrow)$ This part of the proof is essentially the same as in Theorem 2.25 , and it follows from the more general Theorem 2.31, but it is given here to show some special features of partitioning into two pieces. Thus, suppose that $\mathbb{B}$ is not $(\kappa, 2)$-distributive. Let $p \in B^{+}$. By Theorem 1.59 there are partitions $W_{n}$ of $p$ for $n \in \omega$ such that $\left|W_{n}\right|=2$, and if $W_{n}=\left\{a_{n}, b_{n}\right\}$, then for every nonzero $q \leq p$ there is $n \in \omega$ such that neither $q \leq a_{n}$ nor $q \leq b_{n}$ $\left(\left\{a_{n}: n \in \omega\right\}\right.$ is called a splitting family below $p$ ). A winning strategy for White is to play first $p$, and then $a_{n}$ in the $n$-th move.

$(\Rightarrow)$ It follows from Theorem 2.32 that, if White has a winning strategy in the game $\mathcal{G}_{\text {dist }}(\kappa, 2,2)$, then $\mathbb{B}$ is not $\left(\kappa, 2^{<\kappa}\right)$-distributive. But then, by Lemma 1.60 it is not even $(\kappa, 2)$-distributive.

By Theorem 2.10 the existence of an $\omega_{1}$-closed dense subset of $\mathbb{B}$ implies that Black has a winning strategy in $\mathcal{G}_{\text {dist }}(\omega, \infty, 2)$. We have the analogous result for $\mathcal{G}_{\text {dist }}(\kappa, \infty, 2)$ :

Theorem 2.34 If a complete Boolean algebra $\mathbb{B}$ contains a $\lambda$-closed dense subset $D$, then for each infinite cardinal $\kappa<\lambda$ Black has a winning strategy in the game $\mathcal{G}_{\text {dist }}(\kappa, \infty, 2)$.

\footnotetext{
${ }^{12}$ Natasha Dobrinen, contemporary American mathematician
} 
Proof. Let $\kappa<\lambda$. We describe a strategy $\Sigma$ for Black. During the play Black forms a sequence $\left\langle i_{\alpha}: \alpha<\kappa\right\rangle$ in $\nu=$ c.c. $(\mathbb{B})$ and a descending sequence $\left\langle d_{\alpha}: \alpha<\kappa\right\rangle$ of elements of $D$ as follows.

At the beginning, when White plays $p \in B^{+}$and $A_{0} \in \mathcal{A}_{p}(\leq \nu)$, Black chooses $\xi<\nu$ such that $p_{\xi}^{A_{0}} \neq 0$, takes $i_{0}=\Sigma_{p}\left(\left\langle A_{0}\right\rangle\right)=\xi$ and chooses $d_{0} \in D$ satisfying $d_{0} \leq p_{\xi}^{A_{0}}$.

Let $0<\alpha<\kappa$ and let $A_{\beta} \in \mathcal{A}_{p}(\leq \nu)$ (for $\beta<\alpha$ ) be the first $\alpha$ moves of White. Suppose the corresponding choices of Black, $i_{\beta}$ and $d_{\beta}$, are defined for $\beta<\alpha$, so that $d_{0} \geq d_{1} \geq \ldots$ and $d_{\beta} \leq p_{i_{\beta}}^{A_{\beta}}$. In the $\alpha$-th move White chooses $A_{\alpha} \in \mathcal{A}_{p}(\leq \nu)$. Since the set $D$ is $\lambda$-closed and $\alpha<\lambda$, Black may pick $q_{\alpha} \in D$ such that $q_{\alpha} \leq d_{\beta}$ for all $\beta<\alpha$ and choose $i_{\alpha}=\Sigma_{p}\left(\left\langle\left\langle\left\langle A_{\beta}, i_{\beta}\right\rangle\right.\right.\right.$ : $\left.\left.\beta<\alpha\rangle, A_{\alpha}\right\rangle\right) \in \nu$ such that $q_{\alpha} \wedge p_{i_{\alpha}}^{A_{\alpha}}>0$. The set $D$ is dense, so Black chooses $d_{\alpha} \in D$ such that $d_{\alpha} \leq q_{\alpha} \wedge p_{i_{\alpha}}^{A_{\alpha}}$.

Let us prove that $\left\langle\Sigma_{p}: p \in B^{+}\right\rangle$is a winning strategy for Black in $\mathcal{G}_{\text {dist }}(\kappa, \infty, 2)$. Let $\left\langle p, A_{0}, i_{0}, \ldots, A_{\alpha}, i_{\alpha}, \ldots\right\rangle$ be an arbitrary play in which Black follows $\Sigma_{p}$. If $d_{0} \geq d_{1} \geq \ldots$ are elements constructed as above, since the set $D$ is $\lambda$-closed there is $d \in D$ such that $d \leq d_{\alpha}$, for all $\alpha<\kappa$. We have $d_{\alpha} \leq p_{i_{\alpha}}^{A_{\alpha}}$, for all $\alpha<\kappa$, so $\bigwedge_{\alpha \in \kappa} p_{i_{\alpha}}^{A_{\alpha}} \geq \bigwedge_{\alpha \in \kappa} d_{\alpha} \geq d>0$, thus Black wins the game.

In [16] Jech also introduced the games $\mathcal{G}_{\text {dist }}(\omega, \lambda, \omega)$ and $\mathcal{G}_{\text {dist }}(\omega, \infty, \omega)$, in which Black chooses a finite number of elements offered by White instead of only one, as well as $\mathcal{G}_{\text {dist }}\left(\omega, \lambda, \omega_{1}\right)$ and $\mathcal{G}_{\text {dist }}\left(\omega, \infty, \omega_{1}\right)$, in which Black chooses a countable number of elements.

Theorem 2.35 ([16]) If White does not have a winning strategy in the game $\mathcal{G}_{\text {dist }}(\omega, \lambda, \omega)$ played on a complete Boolean algebra $\mathbb{B}$, then it is weakly $(\omega, \lambda)$-distributive.

Theorem 2.36 ([16]) If White does not have a winning strategy in the game $\mathcal{G}_{\text {dist }}(\omega, \infty, \omega)$ played on a complete Boolean algebra $\mathbb{B}$, then $\mathbb{B}$ is weakly $\omega$-distributive.

Concerning the reverse implication of Theorem 2.35, the following theorems were proved by Kamburelis ${ }^{13}$ and Jech:

Theorem 2.37 ([22] Proposition 1.2 and Corollary 1.6) White has a winning strategy in the game $\mathcal{G}_{\text {dist }}(\omega, \lambda, \omega)$ played on a complete Boolean algebra $\mathbb{B}$ iff $\mathbb{B}$ is not $(\omega, \lambda, \omega)$-distributive or $\|\left([\lambda]^{\omega}\right)^{V}$ is non-stationary $\|>0$.

\footnotetext{
${ }^{13}$ Anastasis Kamburelis, contemporary Greek mathematician
} 
Theorem 2.38 ([22]) White has a winning strategy in the game $\mathcal{G}_{\text {dist }}\left(\omega, \omega_{1}\right.$, $\omega)$ played on a complete Boolean algebra $\mathbb{B}$ iff $\mathbb{B}$ is not $\left(\omega, \omega_{1}, \omega\right)$-distributive.

Theorem 2.39 ([16]) If White does not have a winning strategy in the game $\mathcal{G}_{\text {dist }}\left(\omega, \lambda, \omega_{1}\right)$ played on a complete Boolean algebra $\mathbb{B}$, then it is $\left(\omega, \lambda, \omega_{1}\right)$-distributive.

Theorem 2.40 ([16]) If Black has a winning strategy in the game $\mathcal{G}_{\text {dist }}(\omega$, $\left.\infty, \omega_{1}\right)$ played on a complete Boolean algebra $\mathbb{B}$, then $\mathbb{B}$ is proper.

Now we turn to various examples giving us better insight into possible outcomes of the distributivity games.

Example 2.1 (A Boolean algebra on which White wins $\mathcal{G}_{\text {inf }}(\omega)$ but Black wins $\mathcal{G}_{\text {dist }}(\omega, 2,2)$.) Let $\kappa$ be a measurable cardinal and $F$ a normal ultrafilter on $\kappa$. Furthermore, let $\mathbb{P}$ be Prikry forcing (see Example 1.5) and $\mathbb{B}=$ r.o. $(\mathbb{P})$. Then Black has a winning strategy in $\mathcal{G}_{\text {dist }}(\omega, 2,2)$ on $\mathbb{B}$; let us describe this strategy briefly. In the beginning, if White chooses $a \in B^{+}$, Black chooses $\left\langle s, A_{0}\right\rangle \in P$ such that $\left\langle s, A_{0}\right\rangle \leq a$. In each of his next moves Black chooses $i_{n} \in\{0,1\}$ and an element $A_{n+1} \in F$ as follows. Let $\left\langle a, p_{0}, i_{0}, \ldots, p_{n-1}, i_{n-1}, p_{n}\right\rangle$ be a partial play; Black then takes $i_{n} \in\{0,1\}$ and $A_{n+1} \in F$ such that $A_{n+1} \subseteq A_{n}$ and $\left\langle s, A_{n+1}\right\rangle \leq p_{n}^{i_{n}}$ (this can be done by Theorem 1.77). Since $F$ is $\kappa$-complete, $\bigcap_{n \in \omega} A_{n} \in F$ and we have $\left\langle s, \bigcap_{n \in \omega} A_{n}\right\rangle \in P$. Thus $\bigwedge_{n \in \omega} p_{n}^{i_{n}} \geq \bigwedge_{n \in \omega}\left\langle s, A_{n}\right\rangle=\left\langle s, \bigcap_{n \in \omega} A_{n}\right\rangle$.

On the other hand, $\mathbb{B}$ is not $(\omega, \kappa)$-distributive. Hence it is not $\omega$ distributive either, so White has a winning strategy in $\mathcal{G}_{\text {inf }}(\omega)$.

Example 2.2 Assume $\diamond$. There is a Suslin algebra $\mathbb{B}$ (see Example 1.6) such that neither player wins $\mathcal{G}_{\text {dist }}(\omega, 2,2)$ on $\mathbb{B}$. We will generalize this construction in Theorem 3.23.

In [38] a ZFC example of a complete Boolean algebra on which the game $\mathcal{G}_{\text {dist }}(\omega, 2,2)$ is undetermined was obtained.

Lemma 2.41 ([22], Corollary 2.3) There is a complete Boolean algebra that is $\omega$-distributive such that White wins the game $\mathcal{G}_{\text {dist }}\left(\omega, \omega_{2}, \omega_{1}\right)$ played on $\mathbb{B}$.

Example 2.3 (A Boolean algebra on which $\mathcal{G}_{\text {dist }}(\omega, \infty, 2), \mathcal{G}_{\text {dist }}(\omega, \infty, \omega)$ and $\mathcal{G}_{\text {dist }}\left(\omega, \infty, \omega_{1}\right)$ are all undetermined.) Let $A$ be a stationary set in $\omega_{1}$ such that $\omega_{1} \backslash A$ is stationary too, and let $\mathbb{P}_{A}$ be the partial order shooting a club (Example 1.4). $\mathbb{P}_{A}$ is $\omega$-distributive so by Theorem 2.26 and 
Lemma 2.30 White does not win either of games $\mathcal{G}_{\text {dist }}(\omega, \infty, 2) \mathcal{G}_{\text {dist }}(\omega, \infty, \omega)$ or $\mathcal{G}_{\text {dist }}\left(\omega, \infty, \omega_{1}\right)$ on r.o. $(\mathbb{P})$. But since forcing by $\mathbb{P}_{A}$ causes the set $\omega_{1} \backslash A$ to cease being stationary, $\mathbb{P}_{A}$ is not proper and by Theorem 2.40 Black does not have a winning strategy in $\mathcal{G}_{\text {dist }}\left(\omega, \infty, \omega_{1}\right)$, and hence neither in $\mathcal{G}_{\text {dist }}(\omega, \infty, \omega)$ and $\mathcal{G}_{\text {dist }}(\omega, \infty, 2)$.

Example 2.4 (The existence of a winning strategy for Black in $\mathcal{G}_{\text {dist }}(\omega, \infty$, $\omega)$ does not imply $\omega$-distributivity.) Let $\mathbb{B}$ be the random algebra (see Example 1.3). $\mathbb{B}$ is not even $(\omega, 2)$-distributive, but Black has a winning strategy in $\mathcal{G}_{\text {dist }}(\omega, \infty, \omega)$ (see $\left.[16]\right)$.

Thus the game $\mathcal{G}_{\text {dist }}(\omega, \infty, \omega)$ is strictly easier for Black than $\mathcal{G}_{\text {dist }}(\omega, \infty, 2)$ (otherwise the existence of a winning strategy in $\mathcal{G}_{\text {dist }}(\omega, \infty, \omega)$ for Black would imply $\omega$-distributivity) and strictly harder for White (otherwise $\omega$ distributivity would imply the existence of a winning strategy in $\mathcal{G}_{\text {dist }}(\omega, \infty$, $\omega)$ for Black).

\subsection{The distributivity game on partial orders}

In this section we generalize the game $\mathcal{G}_{\text {dist }}(\kappa, 2,2)$ to partial orders. To do this we will combine two notions of a nice name introduced earlier (Definition $1.45)$.

Definition 2.42 A name $\tau \in V^{\mathbb{P}}$ of the form

$$
\tau=\bigcup_{\langle\alpha, \beta\rangle \in \kappa \times \lambda}\left\{\langle\tilde{\alpha, \beta\rangle}\} \times A_{\alpha \beta}\right.
$$

where for each $\alpha<\kappa$

$$
\begin{gathered}
\bigcup_{\beta<\lambda} A_{\alpha \beta} \text { is a maximal antichain in } \mathbb{P} \text { and } \\
A_{\alpha \beta_{1}} \cap A_{\alpha \beta_{2}}=\emptyset \text { for } \beta_{1}<\beta_{2}<\lambda
\end{gathered}
$$

will be called a nice name for a function from $\kappa$ to $\lambda$. The set of all such names will be denoted by $N_{n}^{\mathbb{P}}\left({ }^{\kappa} \lambda\right)$.

Notice that (2.12) also implies that, if $\beta_{1} \neq \beta_{2}$, then $A_{\alpha \beta_{1}}$ and $A_{\alpha \beta_{2}}$ are incompatible.

By $\mathcal{A}(\mathbb{P})$ we will denote the set of all antichains in $\mathbb{P}$. For each $A \in \mathcal{A}(\mathbb{P})$ we fix its "complement", i.e. an antichain $A^{\prime} \in \mathcal{A}(\mathbb{P})$ such that $A \cup A^{\prime}$ is 
a maximal antichain and $A \cap A^{\prime}=\emptyset$ (this also means that $A \perp A^{\prime}$ ). For $n \in\{0,1\}$ denote also

$$
A^{n}= \begin{cases}A & \text { if } n=0 \\ A^{\prime} & \text { if } n=1\end{cases}
$$

The following lemma is a modification of Lemma 1.48.

Lemma 2.43 Let $\mathbb{P}$ be a separative partial ordering, $p \in P$ and $\sigma \in V^{\mathbb{P}}$. Then there is $\tau \in N_{n}^{\mathbb{P}}\left({ }^{\kappa} \lambda\right)$ such that

$$
1 \Vdash \sigma \text { is a function from } \check{\kappa} \text { to } \check{\lambda} \Rightarrow \sigma=\tau \text {. }
$$

Proof. We define $A_{\alpha \beta}$ by recursion on $\langle\alpha, \beta\rangle \in \kappa \times(\lambda \backslash\{0\})$ : let $A_{\alpha \beta}$ be an antichain in $\mathbb{P}$ such that

$$
\begin{array}{r}
\forall p \in A_{\alpha \beta} \quad p \Vdash\langle\alpha, \beta\rangle \in \sigma \\
\forall p \in A_{\alpha \beta} \forall \beta_{1} \quad\left(0<\beta_{1}<\beta \Rightarrow p \perp A_{\alpha \beta_{1}}\right)
\end{array}
$$

and $A_{\alpha \beta}$ is maximal with respect to (2.14) and (2.15). For every $\alpha<\kappa$ let $A_{\alpha 0}$ be $\left(\cup_{0<\beta<\lambda} A_{\alpha \beta}\right)^{\prime}$. Finally, let

$$
\tau=\bigcup_{\langle\alpha, \beta\rangle \in \kappa \times \lambda}\left\{\langle\tilde{\alpha, \beta\rangle}\} \times A_{\alpha \beta}\right.
$$

$\tau$ is a nice name: it is clear that $A_{\alpha \beta_{1}} \cap A_{\alpha \beta_{2}}=\emptyset$ for all $\alpha<\kappa$ and $\beta_{1}<\beta_{2}<\lambda$, and by definition of $A_{\alpha 0}$ we see that $B_{\alpha}=\bigcup_{\beta<\lambda} A_{\alpha \beta}$ is a maximal antichain.

Let $G$ be a $\mathbb{P}$-generic filter over $V$, and assume that $\sigma_{G}$ is a function from $\kappa$ to $\lambda$. We will prove that $\sigma_{G}=\tau_{G}$.

Claim. For all $p \in A_{\alpha 0} \cap G$ there is $q \in G$ such that $q \leq p$ and $q \Vdash$ $\langle\alpha, 0\rangle \in \sigma$.

Proof. We use Lemma 1.35. Suppose that there exists $p \in A_{\alpha 0} \cap G$ that does not satisfy the desired condition. Let $p_{1} \in G$ be such that

$$
p_{1} \Vdash \sigma \text { is a function from } \check{\kappa} \text { to } \check{\lambda} \text {. }
$$

Let $q \in G$ be such that $q \leq p$ and $q \leq p_{1}$. Then

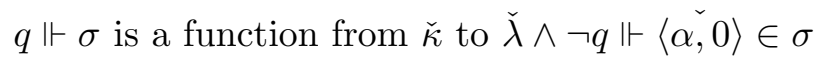


hence there is $r \leq q$ such that $r \Vdash\langle\alpha \check{\alpha, 0}\rangle \notin \sigma$. Since $q \Vdash \exists \beta\langle\check{\lambda}\langle\check{\alpha}, \beta\rangle \in \sigma$, there are $s \leq r$ and $\beta \in \lambda \backslash\{0\}$ such that $s \Vdash\langle\alpha, \beta\rangle \in \sigma$. But $s$ must be incompatible with all antichains $A_{\alpha \gamma}$ for $0<\gamma \leq \beta$ (since $p$ is), which contradicts the maximality of $A_{\alpha \beta}$. This proves the claim.

Next, we prove that $\sigma_{G} \subseteq \tau_{G}$. Let $\langle\alpha, \beta\rangle \in \sigma_{G}$; we will prove $\langle\alpha, \beta\rangle \in \tau_{G}$. It suffices to prove that there is $p \in G \cap A_{\alpha \beta}$, which implies $p \in G$ and $p \Vdash\langle\alpha, \beta\rangle \in \tau$, and hence $\langle\alpha, \beta\rangle \in \tau_{G}$. But, since $B_{\alpha}$ is a maximal antichain, $B_{\alpha} \downarrow$ is dense, so there is $p \in G \cap B_{\alpha}$. If $p$ would be in any $A_{\alpha \beta^{\prime}}$ for some $\beta^{\prime} \neq \beta$ and $\beta^{\prime} \neq 0$, we would have $p \Vdash\left\langle\alpha, \beta^{\prime}\right\rangle \in \sigma$, and thus $\left\langle\alpha, \beta^{\prime}\right\rangle \in \sigma_{G}$, a contradiction. On the other hand, if $p \in A_{\alpha 0}$ and $\beta \neq 0$, then by Claim there is $q \in G$ such that $q \leq p$ and $q \Vdash\langle\alpha, 0\rangle \in \sigma$, so $\langle\alpha, 0\rangle \in \sigma_{G}$, a contradiction again. Hence $p \in A_{\alpha \beta}$ and we proved $\sigma_{G} \subseteq \tau_{G}$.

If $\beta \neq 0$, it is obvious that $\tau_{G} \subseteq \sigma_{G}$ : if $\langle\alpha, \beta\rangle \in \tau_{G}$ then there is $p \in G \cap A_{\alpha \beta}$, but then $p \Vdash\langle\alpha, \beta\rangle \in \sigma$. If $\beta=0$, again by Claim there is $q \in G$ such that $q \leq p$ and $q \Vdash\langle\alpha, 0\rangle \in \sigma$. In both cases, we get $\langle\alpha, \beta\rangle \in \sigma_{G}$.

Now, as in the corollary of Lemma 1.48 we conclude that every function mapping $\kappa$ to $\lambda$ in $V[G]$ has a name in $N_{n}^{\mathbb{P}}\left({ }^{\kappa} \lambda\right)$.

In the proof of our first theorem we will need the following lemmas.

Lemma 2.44 Let $\tau=\bigcup_{\langle\alpha, \beta\rangle \in \kappa \times \lambda}\{\langle\alpha, \beta\rangle\} \times A_{\alpha \beta} \in N_{n}^{\mathbb{P}}\left({ }^{\kappa} \lambda\right)$. Then for every $r \in P$ the following conditions are equivalent:

(i) $r \Vdash\langle\alpha, \beta\rangle \in \tau$

(ii) the set $A_{\alpha \beta} \downarrow$ is dense below $r$.

Proof. (i) $\Rightarrow$ (ii) Suppose $r \Vdash\langle\alpha, \beta\rangle \in \tau$. Let $s \leq r$ and let $G$ be a $\mathbb{P}$-generic filter over $V$ such that $s \in G$. Then $r \in G$, so (i) implies $\langle\alpha, \beta\rangle \in \tau_{G}$. This means that there is $a \in A_{\alpha \beta} \cap G$. Since $s, a \in G$, there is $u \in G$ such that $u \leq s$ and $u \leq a$. Thus we found $u \in A_{\alpha \beta} \downarrow$ such that $u \leq s$.

(ii) $\Rightarrow$ (i) Suppose $A_{\alpha \beta} \downarrow$ is dense below $r$. Let $G$ be any $\mathbb{P}$-generic filter over $V$ such that $r \in G$. Then there exists $s \in G \cap A_{\alpha \beta} \downarrow$ which implies there is $a \in A_{\alpha \beta}$ such that $s \leq a$. Then $a \in G$ as well, so $\langle\alpha, \beta\rangle \in \tau_{G}$. We showed that $r \in G$ implies $\|\langle\alpha, \beta\rangle \in \tau\| \in G$, so we have $r \Vdash\langle\alpha, \beta\rangle \in \tau$.

Lemma 2.45 Let $\tau=\bigcup_{\langle\alpha, \beta\rangle \in \kappa \times \lambda}\{\langle\alpha, \beta\rangle\} \times A_{\alpha \beta} \in N_{n}^{\mathbb{P}}\left({ }^{\kappa} \lambda\right)$. Then for every $r \in P$ the following conditions are equivalent: 
(i) $r \Vdash\langle\alpha, \beta\rangle \notin \tau$

(ii) the set $A_{\alpha \beta} \downarrow$ is empty below $r$.

Proof. (i) $\Rightarrow$ (ii) Let $r \Vdash\langle\alpha, \beta\rangle \notin \tau$. Suppose there is $s \leq r$ such that $s \in$ $A_{\alpha \beta} \downarrow$. Then there is $a \in A_{\alpha \beta}$ such that $s \leq a$. This implies $a \Vdash\langle\alpha, \beta\rangle \in \tau$, and hence $s \Vdash\langle\alpha, \beta\rangle \in \tau$. On the other hand, $s \leq r$ and $r \Vdash\langle\alpha, \beta\rangle \notin \tau$ implies $s \Vdash\langle\alpha, \beta\rangle \notin \tau$, a contradiction.

(ii) $\Rightarrow$ (i) Suppose $A_{\alpha \beta} \downarrow$ is empty below $r$ and not $r \Vdash\langle\alpha, \beta\rangle \notin \tau$. Then there is $q \leq r$ such that $q \Vdash\langle\alpha, \beta\rangle \in \tau$. But then by Lemma $2.44 A_{\alpha \beta} \downarrow$ is dense below $q$, so there is $s \in A_{\alpha \beta} \downarrow$ such that $s \leq q \leq r$, a contradiction.

Lemma 2.46 Let $A$ be an antichain in $\mathbb{P}$ and let $p \in P$. Then

$A \downarrow$ is dense below $p$ iff $A^{\prime} \downarrow$ is empty below $p$.

Proof. $(\Rightarrow)$ Suppose $A \downarrow$ is dense below $p$, but there is $q \leq p$ such that $q \in A^{\prime} \downarrow$. Then there is $u \in A \downarrow$ such that $u \leq q$, i.e. there is $a \in A$ such that $u \leq a$, but also there is $b \in A^{\prime}$ such that $q \leq b$. We have $u \leq a$ and $u \leq b$, a contradiction with the fact that $A \perp A^{\prime}$.

$(\Leftarrow)$ Suppose $A^{\prime} \downarrow$ is empty below $p$, but there is $q \leq p$ such that there are no elements of $A \downarrow$ below $q$. But then $A \cup A^{\prime} \cup\{q\}$ is an antichain, a contradiction with the maximality of the antichain $A \cup A^{\prime}$.

Lemma 2.47 If $S$ is any set and $\varphi(x), \psi(x)$ are formulas, then the following conditions are equivalent:

(i) $\forall A \subseteq S(\exists a \in A \varphi(a) \vee \exists a \in S \backslash A \psi(a))$

(ii) $\exists a \in S(\varphi(a) \wedge \psi(a))$.

Proof. (i) $\Rightarrow$ (ii) Let (i) hold, and $A=\{s \in S: \neg \varphi(s)\}$. By (i) there is $s_{0} \in S \backslash A$ such that $\psi\left(s_{0}\right)$. But $\varphi\left(s_{0}\right)$ holds as well, since $s_{0} \notin A$.

(ii) $\Rightarrow$ (i) Let $s_{0} \in S$ be such that $\varphi\left(s_{0}\right)$ and $\psi\left(s_{0}\right)$, and $A \subseteq S$. If $s_{0} \in A$ then $\exists a \in A \varphi(a)$; otherwise $\exists s \in S \backslash A \psi(s)$.

Theorem 2.48 Let $\mathbb{P}$ be a separative partial ordering. r.o. $(\mathbb{P})$ is not $(\kappa, \lambda)$ distributive iff there is a family $\left\{A_{\alpha \beta}:\langle\alpha, \beta\rangle \in \kappa \times \lambda\right\}$ such that (2.12) holds and there is $p \in P$ such that

$\forall q \leq p \exists\langle\alpha, \beta\rangle \in \kappa \times \lambda\left(A_{\alpha \beta} \downarrow\right.$ is neither dense nor empty below $\left.q\right)$. (2.16) 
Proof. From Theorem 1.59 it follows that r.o. $(\mathbb{P})$ is not $(\kappa, \lambda)$-distributive iff in some extension $V[G]$ via $\mathbb{P}$ there is a new function from $\kappa$ to $\lambda$.

$(\Rightarrow)$ Let $V[G]$ be a generic extension containing a new function from $\kappa$ to $\lambda$ and let $\tau=\bigcup_{\langle\alpha, \beta\rangle \in \kappa \times \lambda}\{\langle\alpha, \beta\rangle\} \times A_{\alpha \beta}$ be its nice name. Then (2.12) clearly holds, and there is $p \in G$ such that $p \Vdash \forall f \subseteq\left((\kappa \times \lambda)^{V}\right)^{r} f \neq \tau$ (i.e. $p$ forces that $\tau_{G}$ is not in $V$ ), so using Lemmas 2.47, 2.44 and 2.45 we have

$$
\begin{array}{ll} 
& p \Vdash \forall f \subseteq\left((\kappa \times \lambda)^{V}\right)^{r} f \neq \tau \\
\Leftrightarrow & \forall f \subseteq \kappa \times \lambda \neg \exists q \leq p q \Vdash \check{f}=\tau \\
\Leftrightarrow & \forall q \leq p \forall f \subseteq \kappa \times \lambda \\
& \neg q \Vdash(\forall\langle\alpha, \beta\rangle \in \check{f}\langle\alpha, \beta\rangle \in \tau \wedge \forall\langle\alpha, \beta\rangle \in(\check{\kappa} \times \check{\lambda}) \backslash \check{f}\langle\alpha, \beta\rangle \notin \tau) \\
\Leftrightarrow & \forall q \leq p \forall f \subseteq \kappa \times \lambda \neg(\forall\langle\alpha, \beta\rangle \in f q \Vdash\langle\alpha, \beta\rangle \in \tau \\
& \wedge \forall\langle\alpha, \beta\rangle \in(\kappa \times \lambda) \backslash f q \Vdash\langle\alpha, \beta\rangle \notin \tau) \\
\Leftrightarrow & \forall q \leq p \forall f \subseteq \kappa \times \lambda(\exists\langle\alpha, \beta\rangle \in f \neg q \Vdash\langle\alpha, \beta\rangle \in \tau \\
& \vee \exists\langle\alpha, \beta\rangle \in(\kappa \times \lambda) \backslash f \neg q \Vdash\langle\alpha, \beta\rangle \notin \tau) \\
\Leftrightarrow & \forall q \leq p \exists\langle\alpha, \beta\rangle \in \kappa \times \lambda(\neg q \Vdash\langle\alpha, \beta\rangle \in \tau \wedge \neg q \Vdash\langle\alpha, \beta\rangle \notin \tau) \\
\Leftrightarrow & \forall q \leq p \exists\langle\alpha, \beta\rangle \in \kappa \times \lambda \\
& \left(A_{\alpha \beta \downarrow} \downarrow \text { is neither dense nor empty below } q\right) .
\end{array}
$$

Thus condition (2.16) is satisfied.

$(\Leftarrow)$ Suppose there are a family $\left\{A_{\alpha \beta}:\langle\alpha, \beta\rangle \in \kappa \times \lambda\right\} \subseteq \mathcal{A}(\mathbb{P})$ and $p \in P$ satisfying (2.12) and (2.16). Then $\tau=\bigcup_{\langle\alpha, \beta\rangle \in \kappa \times \lambda}\{\langle\alpha, \beta\rangle\} \times A_{\alpha \beta} \in N_{n}^{\mathbb{P}}\left({ }^{\kappa} \lambda\right)$. From the equivalence proved in $(\Rightarrow)$ we deduce that $p$ forces that $\tau_{G}$ is not in $V$. In other words, forcing with r.o. $(\mathbb{P})$ can add a function mapping $\kappa$ to $\lambda$, so r.o. $(\mathbb{P})$ is not $(\kappa, \lambda)$-distributive.

Definition 2.49 A separative partial ordering $\mathbb{P}$ is $(\kappa, \lambda)$-distributive iff for every family $\left\{A_{\alpha \beta}:\langle\alpha, \beta\rangle \in \kappa \times \lambda\right\}$ satisfying (2.12) the set

$$
\left\{p \in P: \forall\langle\alpha, \beta\rangle \in \kappa \times \lambda\left(A_{\alpha \beta} \downarrow \text { is dense or empty below } p\right)\right\}
$$

is dense in $\mathbb{P}$.

By Theorem 2.48 we have a simple result that explains the definition above:

Lemma 2.50 A partial order $\mathbb{P}$ is $(\kappa, \lambda)$-distributive iff r.o. $(\mathbb{P})$ is.

Corollary 2.51 If $\mathbb{P}$ is $(\kappa, 2)$-distributive, then it is also $\left(\kappa, 2^{\kappa}\right)$-distributive. 
Proof. If $\mathbb{P}$ is $(\kappa, 2)$-distributive, then r.o. $(\mathbb{P})$ is $(\kappa, 2)$-distributive, so by Lemma 1.60 r.o. $(\mathbb{P})$ is also $\left(\kappa, 2^{\kappa}\right)$-distributive. Thus $\mathbb{P}$ is $\left(\kappa, 2^{\kappa}\right)$-distributive.

Now we define $\mathcal{G}_{\text {dist }}^{\prime}(\kappa)$, the distributivity game on partial orders:

Definition 2.52 The game $\mathcal{G}_{\text {dist }}^{\prime}(\kappa)$ is played in $\kappa$-many steps on a partial order $\mathbb{P}$ as follows:

- first White chooses an element $p \in P$; after that the players make their moves subsequently:

- in his $\alpha$-th move White chooses $A_{\alpha} \in \mathcal{A}(\mathbb{P})$;

- in his $\alpha$-th move Black chooses $i_{\alpha} \in\{0,1\}$.

Black wins if

$$
\exists q \leq p \forall \alpha<\kappa\left(A_{\alpha}^{i_{\alpha}} \downarrow \text { is dense below } q\right),
$$

and White wins otherwise.

Of course, we can also deal only with antichains below $p$, but it would not make the situation any simpler.

A winning strategy for White consists of $p \in P$ and a function mapping every sequence of previous moves $\left\langle\left\langle A_{\alpha}, i_{\alpha}\right\rangle: \alpha<\mu\right\rangle$ to an antichain in $\mathbb{P}$. However, it is easy to prove a theorem analogous to Theorem 2.21:

Theorem 2.53 For every partial order $\mathbb{P}$ the following conditions are equivalent:

(a) White has a winning strategy in the game $\mathcal{G}_{\text {dist }}^{\prime}(\kappa)$ played on $\mathbb{P}$;

(b) There are $p \in P$ and $w:{ }^{<\kappa} 2 \rightarrow \mathcal{A}(\mathbb{P})$ such that

$$
\forall i \in{ }^{\kappa} 2 \forall q \leq p \exists \alpha<\kappa\left(w(i\lceil\alpha))^{i(\alpha)} \downarrow \text { is not dense below } q\right) \text {. }
$$

Definition 2.54 If $A_{1}$ and $A_{2}$ are antichains in $\mathbb{P}$, let us say that $A_{1}$ is below $A_{2}$ (and write $A_{1} \triangleleft A_{2}$ ) if for each $a_{1} \in A_{1} \downarrow$ there is $a_{2} \in A_{2} \downarrow$ such that $a_{2} \leq a_{1}$.

The next lemma explains the term "below": 
Lemma 2.55 $A_{1} \triangleleft A_{2}$ iff (in $\mathbb{B}=$ r.o. $(\mathbb{P})$ ) $\bigvee A_{1} \leq \bigvee A_{2}$

Proof. Let $A_{1} \triangleleft A_{2}$, and suppose that $\bigvee A_{1} \leq \bigvee A_{2}$ doesn't hold. Then the element $b=\bigvee A_{1} \wedge\left(\bigvee A_{2}\right)^{\prime}$ of $B$ is compatible with at least one element of $A_{1}$, say $a$. Since $P$ is dense in r.o. $(\mathbb{P})$, there is $p \in P$ such that $p \leq b \wedge a$. But then $p \in A_{1} \downarrow$ and $p$ is incompatible with $A_{2}$, a contradiction.

Now let $\bigvee A_{1} \leq \bigvee A_{2}$ and $a_{1} \in A_{1} \downarrow$. It follows that $a_{1} \leq \bigvee A_{1} \leq \bigvee A_{2}$, so there is $a_{2} \in A_{2}$ compatible with $a_{1}$, which means that there is $a_{3}$ such that $a_{3} \leq a_{2}\left(\right.$ so $\left.a_{3} \in A_{2} \downarrow\right)$ and $a_{3} \leq a_{1}$.

Lemma 2.56 Let $A_{1}, A_{2}, B_{1}$ and $B_{2}$ be antichains in $\mathbb{P}$.

(a) $A_{1}$ and $A_{2}$ are incompatible iff (in r.o.(P)) $\bigvee A_{1} \wedge \bigvee A_{2}=0$

(b) If $A_{1}$ and $A_{2}$ are incompatible, $B_{1} \triangleleft A_{1}$ and $B_{2} \triangleleft A_{2}$, then $B_{1}$ and $B_{2}$ are incompatible.

Proof. (a) Applying distributivity laws in Boolean algebras, we get $\bigvee A_{1} \wedge$ $\bigvee A_{2}=\bigvee_{a_{1} \in A_{1}} a_{1} \wedge \bigvee_{a_{2} \in A_{2}} a_{2}=\bigvee_{a_{1} \in A_{1}}\left(a_{1} \wedge \bigvee_{a_{2} \in A_{2}} a_{2}\right)=\bigvee_{a_{1} \in A_{1}, a_{2} \in A_{2}}\left(a_{1} \wedge\right.$ $a_{2}$ ), which is 0 iff all elements $a_{1} \wedge a_{2}$ are equal to 0 , i.e. iff $A_{1}$ and $A_{2}$ are incompatible.

(b) Follows directly from (a) and Lemma 2.55.

Theorem 2.57 The following conditions are equivalent for every separative partial ordering $\mathbb{P}$ :

(i) $\mathbb{P}$ is not $(\kappa, 2)$-distributive;

(ii) in some extension $V[G]$ by $\mathbb{P}$ there is a new function $f: \kappa \rightarrow 2$;

(iii) White has a winning strategy in the game $\mathcal{G}_{\text {dist }}^{\prime}(\kappa)$ played on $\mathbb{P}$.

Proof. (i) $\Leftrightarrow$ (ii) Follows from Lemma 2.50 and Theorem 1.59.

(ii) $\Rightarrow$ (iii) Let $f: \kappa \rightarrow 2$ be a new function in some extension $V[G]$, and let $\tau=\bigcup_{\langle\alpha, \beta\rangle \in \kappa \times 2}\{\langle\alpha, \beta\rangle\} \times B_{\alpha \beta}$ be its nice name. This means that there is $p \in P$ such that for all $g \in\left({ }^{\kappa} 2\right)^{V} p \Vdash \tau \neq \check{g}$. We define a function $w:{ }^{<} 2 \rightarrow \mathcal{A}(\mathbb{P})$ by $w(\varphi)=B_{\alpha 0}$, where $\alpha=\operatorname{dom} \varphi$. (Thus the strategy of White does not depend on Black's previous moves.) We will prove that (2.18) holds, so Theorem 2.53 will imply (iii). Suppose the opposite, that (2.18) does not hold; then there are $i: \kappa \rightarrow 2$ and $q \leq p$ such that for all $\alpha<\kappa$ the set $B_{\alpha i(\alpha)} \downarrow$ is dense below $q$. Then Lemma 2.44 implies that $q \Vdash\langle\alpha, i(\alpha)\rangle \in \tau$ holds for all $\alpha<\kappa$. This means that $q \Vdash \tau=\check{i}$, and therefore $p \Vdash \tau \neq \check{i}$ doesn't hold; a contradiction, since $i$ is a function in $V$. 
(iii) $\Rightarrow$ (i) In this part of the proof we modify the idea from Theorem 2.25. Let $p$ and $w$ be the objects provided by Theorem 2.53. We define a family $\left\{\left\{B_{\varphi}^{0}, B_{\varphi}^{1}\right\}: \varphi \in{ }^{<\kappa} 2\right\}$ of pairs of antichains in $\mathbb{P}$ in the following way:

- first, let $B_{\emptyset}^{0}=w_{\emptyset}$ and $B_{\emptyset}^{1}=w_{\emptyset}^{\prime}$;

- for each $\varphi \in{ }^{<\kappa} 2$, if $\operatorname{dom} \varphi=\alpha$ and we defined $B_{\varphi \uparrow \beta}^{n}$ for all $\beta<\alpha$ and $n \in\{0,1\}$, let $B_{\varphi}^{0}$ be a maximal antichain in $\mathbb{P}$ such that $B_{\varphi}^{0} \triangleleft w_{\varphi}$ and $B_{\varphi}^{0} \triangleleft B_{\varphi \mid \beta}^{\varphi(\beta)}$ for $\beta<\alpha$. (If there is no such antichain, i.e. if in r.o.(P) $\bigvee w_{\varphi} \wedge \wedge_{\beta<\alpha} \bigvee B_{\varphi \uparrow \beta}^{\varphi(\beta)}=0$, we take $B_{\varphi}^{0}=\emptyset$.) We define $B_{\varphi}^{1}$ in a similar way: it is a maximal antichain in $\mathbb{P}$ such that $B_{\varphi}^{1} \triangleleft w_{\varphi}^{\prime}$ and $B_{\varphi}^{1} \triangleleft B_{\varphi \uparrow \beta}^{\varphi(\beta)}$ for $\beta<\alpha$.

Claim 1. Let $\alpha<\kappa, \varphi, \varphi^{\prime} \in{ }^{\alpha} 2$ and $\varphi \neq \varphi^{\prime}$. Then: (a) $B_{\varphi}^{0} \perp B_{\varphi}^{1}$; (b) if $n_{1}, n_{2} \in\{0,1\}$, then $B_{\varphi}^{n_{1}} \perp B_{\varphi^{\prime}}^{n_{2}}$; (c) $\bigcup_{\varphi \in \epsilon_{2}}\left(B_{\varphi}^{0} \cup B_{\varphi}^{1}\right)$ is an antichain in $\mathbb{P}$.

Proof. (a) Since $B_{\varphi}^{0} \triangleleft w_{\varphi}^{0}$ and $B_{\varphi}^{1} \triangleleft w_{\varphi}^{1}$ and $w_{\varphi}^{0}$ and $w_{\varphi}^{1}$ are incompatible, Lemma 2.56(b) implies that $B_{\varphi}^{0}$ and $B_{\varphi}^{1}$ are incompatible too.

(b) We prove this by induction on $\alpha$. Assume it holds for all $\beta<\alpha$. If $\varphi \neq \varphi^{\prime}$, let $\gamma$ be the smallest ordinal such that $\varphi(\gamma) \neq \varphi^{\prime}(\gamma)$. Then $B_{\varphi}^{n_{1}} \triangleleft B_{\varphi \mid \gamma}^{\varphi(\gamma)}$ and $B_{\varphi^{\prime}}^{n_{2}} \triangleleft B_{\varphi^{\prime} \mid \gamma}^{\varphi^{\prime}(\gamma)}$. By (a) $B_{\varphi \mid \gamma}^{\varphi(\gamma)}$ and $B_{\varphi^{\prime} \mid \gamma}^{\varphi^{\prime}(\gamma)}$ are incompatible, so by Lemma 2.56(b) $B_{\varphi}^{n_{1}}$ and $B_{\varphi^{\prime}}^{n_{2}}$ are incompatible too.

(c) Now $\bigcup_{\varphi \in \in_{2}}\left(B_{\varphi}^{0} \cup B_{\varphi}^{1}\right)$ is union of pairwise incompatible antichains, so it is an antichain too.

Claim 2. If $\alpha_{1}<\alpha_{2}<\kappa, \varphi_{1} \in{ }^{\alpha_{1}} 2, \varphi_{2} \in{ }^{\alpha_{2}} 2$ and $n_{1}, n_{2} \in\{0,1\}$ then

$$
B_{\varphi_{2}}^{n_{2}} \triangleleft B_{\varphi_{1}}^{n_{1}} \text { iff } \varphi_{1}=\varphi_{2}\left\lceil\alpha_{1} \text { and } \varphi_{2}\left(\alpha_{1}\right)=n_{1},\right.
$$

and if this is not the case, then $B_{\varphi_{1}}^{n_{1}}$ and $B_{\varphi_{2}}^{n_{2}}$ are incompatible.

Proof. First let $\varphi_{1}=\varphi_{2}\left\lceil\alpha_{1}\right.$ and $\varphi_{2}\left(\alpha_{1}\right)=n_{1}$. Then $B_{\varphi_{2}}^{n_{2}}$ is defined so that $B_{\varphi_{2}}^{n_{2}} \triangleleft B_{\varphi_{1}}^{n_{1}}$.

Now suppose $\varphi_{1} \neq \varphi_{2}\left\lceil\alpha_{1}\right.$, and let $\gamma<\alpha_{1}$ be the smallest ordinal such that $\varphi_{1}(\gamma) \neq \varphi_{2}(\gamma)$. Then by the definition above $B_{\varphi_{1}}^{n_{1}}$ is below $B_{\varphi_{1} \mid \gamma}^{\varphi_{1}(\gamma)}$ and $B_{\varphi_{2}}^{n_{2}}$ is below $B_{\varphi_{2} \mid \gamma}^{\varphi_{2}(\gamma)}$, but $B_{\varphi_{1} \mid \gamma}^{\varphi_{1}(\gamma)}$ and $B_{\varphi_{2} \mid \gamma}^{\varphi_{2}(\gamma)}$ are incompatible by Claim 1(b), since $\varphi_{1}\left\lceil\gamma=\varphi_{2} \uparrow \gamma\right.$ and $\varphi_{1}(\gamma) \neq \varphi_{2}(\gamma)$. Thus, by Lemma 2.56(b), $B_{\varphi_{1}}^{n_{1}}$ and $B_{\varphi_{2}}^{n_{2}}$ are incompatible as well.

Finally, let $\varphi_{1}=\varphi_{2}\left\lceil\alpha_{1}\right.$ and $\varphi_{2}\left(\alpha_{1}\right) \neq n_{1}$. Then $B_{\varphi_{2}}^{n_{2}}$ is below $B_{\varphi_{1}}^{\varphi_{2}\left(\alpha_{1}\right)}$, so it must be incompatible with $B_{\varphi_{1}}^{n_{1}}$. The claim is proved.

There are two possibilities: 
$1^{\circ} \bigcup_{\varphi \in \alpha_{2}}\left(B_{\varphi}^{0} \cup B_{\varphi}^{1}\right)$ is a maximal antichain for every $\alpha<\kappa$. Suppose $\mathbb{P}$ is $(\kappa, 2)$-distributive. Then, by Corollary 2.51 it is also $\left(\kappa, 2^{\kappa}\right)$ distributive, and hence $\left(\kappa, 2^{<\kappa}\right)$-distributive as well. For all $\alpha<\kappa$ enumerate $\left\{B_{\varphi}^{n}: \varphi \in{ }^{\alpha} 2 \wedge n \in\{0,1\}\right\}$ as $\left\{C_{\alpha \beta}: \beta<2^{|\alpha|}\right\}$ and take $C_{\alpha \beta}=\emptyset$ whenever $2^{|\alpha|} \leq \beta<2^{<\kappa}$. Applying Theorem 2.48 to the family $\left\{C_{\alpha \beta}: \alpha<\kappa, \beta<\right.$ $\left.2^{<\kappa}\right\}$ and $p$ we deduce that there is $q \leq p$ such that for all $\langle\alpha, \beta\rangle \in \kappa \times 2^{<\kappa}$ the set $C_{\alpha \beta}$ is either dense or empty below $q$.

Claim 3. For each $\alpha<\kappa$ there is exactly one $\beta<2^{<\kappa}$ such that $C_{\alpha \beta} \downarrow$ is dense below $q$.

Proof. Since $\bigcup_{\beta<2<\kappa} C_{\alpha \beta}$ is a maximal antichain, not all $C_{\alpha \beta} \downarrow$ can be empty below $q$. On the other hand, if $C_{\alpha \beta_{0}} \downarrow$ is dense below $q$, then by

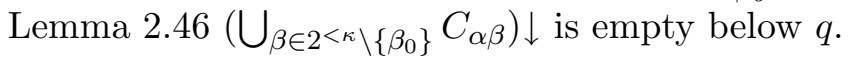

For each $\alpha<\kappa$ let $\beta(\alpha)$ be such that $C_{\alpha \beta(\alpha)} \downarrow$ is dense below $q$.

Claim 4. If $\alpha_{1}<\alpha_{2}<\kappa$, then $C_{\alpha_{2} \beta\left(\alpha_{2}\right)}$ is below $C_{\alpha_{1} \beta\left(\alpha_{1}\right)}$.

Proof. Claim 2 implies that every two antichains $C_{\alpha_{2} \beta\left(\alpha_{2}\right)}$ and and $C_{\alpha_{1} \beta\left(\alpha_{1}\right)}$ are either incompatible, or the former is below the latter. But it is impossible that $C_{\alpha_{2} \beta\left(\alpha_{2}\right)}$ and $C_{\alpha_{1} \beta\left(\alpha_{1}\right)}$ are incompatible: take $b_{1} \in C_{\alpha_{1} \beta\left(\alpha_{1}\right)} \downarrow$ such that $b_{1} \leq q$ and $b_{2} \in C_{\alpha_{2} \beta\left(\alpha_{2}\right) \downarrow} \downarrow$ such that $b_{2} \leq b_{1}$, then $b_{2} \in C_{\alpha_{1} \beta\left(\alpha_{1}\right) \downarrow}$ $\cap C_{\alpha_{2} \beta\left(\alpha_{2}\right) \downarrow}$. So $C_{\alpha_{2} \beta\left(\alpha_{2}\right)}$ must be below $C_{\alpha_{1} \beta\left(\alpha_{1}\right)}$.

Finally, define a function $i: \kappa \rightarrow 2$ and a sequence $\left\langle\varphi_{\alpha}: \alpha<\kappa\right\rangle$ in the following way: if $\alpha<\kappa$, let $\varphi_{\alpha} \in{ }^{\alpha} 2$ and $i(\alpha) \in\{0,1\}$ be such that $C_{\alpha \beta(\alpha)}=B_{\varphi_{\alpha}}^{i(\alpha)}$. We prove by induction that $\varphi_{\alpha}=i \uparrow \alpha$ for all $\alpha<\kappa$. For $\varphi_{0}=\langle\rangle$ this is obvious. Suppose $\varphi_{\gamma}=i \uparrow \gamma$ for all $\gamma<\alpha$. By Claims 4 and 2 , if $B_{\varphi_{\gamma}}^{i(\gamma)}=C_{\gamma \beta(\gamma)}$ and $B_{\varphi_{\alpha}}^{i(\alpha)}=C_{\alpha \beta(\alpha)}$, then $\varphi_{\gamma}=\varphi_{\alpha} \uparrow \gamma$. Thus, $\varphi_{\gamma}=i \uparrow \gamma$ implies $\varphi_{\alpha} \uparrow \gamma=i \uparrow \gamma$. If $\alpha$ is a limit ordinal, it follows that $\varphi_{\alpha}=\bigcup_{\gamma<\alpha}\left(\varphi_{\alpha} \uparrow \gamma\right)=\bigcup_{\gamma<\alpha}(i \uparrow \gamma)=i \uparrow \alpha$. If $\alpha=\gamma+1$, then by definition of $i$ and Claim 2, $\varphi_{\gamma}=i(\gamma)$. Hence $\varphi_{\alpha}=i \uparrow \alpha$.

Furthermore, from the definition of the family $\left\{\left\{B_{\varphi}^{0}, B_{\varphi}^{1}\right\}: \varphi \in{ }^{<\kappa} 2\right\}$ we see that $B_{i \uparrow \alpha}^{i(\alpha)}$ is below $w_{i \uparrow \alpha}^{i(\alpha)}$. Thus $w_{i \uparrow \alpha}^{i(\alpha)}$ is also dense below $q$ for $\alpha<\kappa$, so White doesn't win the game, a contradiction.

$2^{\circ} \bigcup_{\varphi \in{ }^{\alpha} 2}\left(B_{\varphi}^{0} \cup B_{\varphi}^{1}\right)$ is not maximal for some $\alpha<\kappa$. Let $\alpha_{0}$ be the least such $\alpha$ and $D=\bigcup_{\varphi \in{ }^{\alpha} 0}\left(B_{\varphi}^{0} \cup B_{\varphi}^{1}\right)$. We prove that then $\mathbb{P}$ is not even $\left(\left|\alpha_{0}\right|, 2^{<\kappa}\right)$-distributive.

Suppose it is not so. Let $p^{\prime} \in P$ be such that $p^{\prime}$ is incompatible with $D$. 
After defining $C_{\alpha \beta}$ for $\alpha<\alpha_{0}$ and $\beta<2^{<\kappa}$ as in $1^{\circ}$, we then apply Theorem 2.48 to family $\left\{C_{\alpha \beta}: \alpha<\alpha_{0} \wedge \beta<2^{<\kappa}\right\}$ and $p^{\prime}$, and find $q \leq p^{\prime}$ such that all $C_{\alpha \beta}$ are either dense or empty below $q$. Again, as in $1^{\circ}$, we find $C_{\alpha \beta(\alpha)}$ for $\alpha<\alpha_{0}$, all dense below $q$, and a function $i: \alpha_{0} \rightarrow 2$ such that $C_{\alpha \beta(\alpha)}=B_{i \uparrow \alpha}^{i(\alpha)}$ for all $\alpha<\alpha_{0}$. Since $w_{i \uparrow \alpha_{0}}^{0} \cup w_{i \uparrow \alpha_{0}}^{1}$ is a maximal antichain, $q$ can not be incompatible with both $w_{i\left\lceil\alpha_{0}\right.}^{0}$ and $w_{i\left\lceil\alpha_{0}\right.}^{1}$, so it is compatible with at least one of the antichains $B_{i \uparrow \alpha_{0}}^{0}$ and $B_{i \uparrow \alpha_{0}}^{1}$. This means that $p^{\prime}$ is not incompatible with all antichains constructed in $\alpha$-th step, a contradiction.

\subsection{The games $\mathcal{G}_{\mathrm{sp}}, \mathcal{G}_{\mathrm{b}}$ and $\mathcal{G}_{\mathrm{lp}}$}

Now we consider three more cut-and-choose games, introduced by Kada ${ }^{14}$ in [21], where he stated all the results stated here. We provide detailed proofs of these results.

Definition 2.58 $\mathcal{G}_{\mathrm{sp}}$ is the cut-and-choose game of type $(\omega, \omega, \mu)$, where $\mu(n)=n+2$ for $n<\omega$, such that White wins the play $\left\langle p, A_{0}, a_{0}, \ldots, A_{n}\right.$, $\left.a_{n}, \ldots\right\rangle$ iff $\bigwedge_{n<\omega} \bigvee_{j \in a_{n}} p_{j}^{A_{n}}=0$.

From Theorem 2.20 we have (reminding the reader that by $\mathcal{S}$ we denoted the set of slaloms)

Theorem 2.59 For every complete Boolean algebra $\mathbb{B}$ the following conditions are equivalent:

(a) White has a winning strategy in the game $\mathcal{G}_{\mathrm{sp}}$ on $\mathbb{B}$;

(b) there are $p \in B^{+}$and $w: \bigcup_{n<\omega} \prod_{k<n}[\omega] \leq k+1 \rightarrow \mathcal{A}_{p}(\leq \omega)$ such that

$$
\forall \varphi \in \mathcal{S} \bigwedge_{k<\omega} \bigvee_{j \in \varphi(k)} p_{j}^{w(\varphi \mid k)}=0
$$

(c) there are $p \in B^{+}$and $w: \bigcup_{n<\omega} \prod_{k<n}[\omega] \leq k+1 \rightarrow \mathcal{A}_{p}(\omega)$ such that (2.19) holds.

Definition 2.60 By $\mathcal{P}$ we denote the set of all functions mapping $<\omega \omega$ to $\omega$, and by $\mathcal{P}^{*}$ the set of all functions mapping $\bigcup_{n<\omega} \prod_{i<n}[\omega] \leq i+1$ to $\omega$. The elements of both these sets are called predictors.

\footnotetext{
${ }^{14}$ Masaru Kada, contemporary Japanese mathematician
} 
Theorem 2.61 For every complete Boolean algebra $\mathbb{B}$ the following conditions are equivalent:

(i) $\mathbb{B}$ has the Sacks property, i.e. in every generic extension $V[G]$, for every $f \in{ }^{\omega} \omega$ there is $\varphi \in \mathcal{S}^{V}$ such that $f(n) \in \varphi(n)$ for all $n<\omega$;

(ii) in every generic extension $V[G]$, for every predictor $\pi \in \mathcal{P}^{*}$ there is $\varphi \in \mathcal{S}^{V}$ such that $\pi(\varphi \mid n) \in \varphi(n)$ for all $n<\omega$;

(iii) White does not have a winning strategy in $\mathcal{G}_{\text {sp }}$ played on $\mathbb{B}$.

Proof. (i) $\Rightarrow$ (ii) Suppose that (i) holds; let $V[G]$ be a generic extension and $\pi \in\left(\mathcal{P}^{*}\right)^{V[G]}$. In $V$, let $\left\{\sigma_{j}: j<\omega\right\}$ be an arbitrary enumeration of the set $\bigcup_{n<\omega} \prod_{i<n}[\omega] \leq i+1$ (this set is same in $V$ and in $V[G]$, and contains $\emptyset$ as the product of empty family). Furthermore, let $h: \omega \rightarrow \omega$ be a function given by $h(j)=\pi\left(\sigma_{j}\right)$ (since $\pi \in V[G], h$ is also in $V[G]$ ). Since $\mathbb{B}$ has the Sacks property, it is also $\omega^{\omega}$-bounding, so there is $h^{\prime}: \omega \rightarrow \omega$ in $V$ such that $h^{\prime}(j) \geq h(j)$ for all $j \in \omega$. Using recursion on $n$ we define $d(n)=\max \left\{h^{\prime}(j)\right.$ : $\left.\sigma_{j} \in \prod_{i<n}[d(i)]^{\leq i+1}\right\}+1$. Then we define $k_{n}=\left|\bigcup_{m<n} \prod_{i<m}[d(i)]^{\leq i+1}\right|$ and enumerate each set $\prod_{i<n}[d(i)]^{\leq i+1}$ as $\left\{\tau_{j}: k_{n-1} \leq j<k_{n}\right\}$. We obtain an enumeration $\tau=\left\{\tau_{j}: j<\omega\right\}$ of $\bigcup_{n<\omega} \prod_{i<n}[d(i)]^{\leq i+1}$ such that $i<j \Rightarrow$ $\left|\tau_{i}\right| \leq\left|\tau_{j}\right|$. Clearly, this enumeration is itself in $V$.

Now define $g(n)=\left|\tau_{n}\right|+1$; this is a nondecreasing function unbounded in $\omega$. Let $f(j)=\pi\left(\tau_{j}\right)$, and let $\psi$ be the slalom in $\left(\mathcal{S}^{g}\right)^{V}$ such that for all $j \in \omega$ holds $f(j) \in \psi(j)$, provided by (i) and Lemma 1.72. Since $f(j)=$ $\pi\left(\tau_{j}\right)=\pi\left(\sigma_{k}\right)$ for some $k \in \omega$, and $\pi\left(\sigma_{k}\right)=h(k) \leq h^{\prime}(k)<d\left(\left|\sigma_{k}\right|\right)=$ $d\left(\left|\tau_{j}\right|\right)$, we can assume $\psi(j) \subseteq d\left(\left|\tau_{j}\right|\right)$ (if not, we take $\psi(j) \cap d\left(\left|\tau_{j}\right|\right)$ instead of $\psi(j))$. We define $\varphi$ by recursion: $\varphi(n)=\psi(j)$, where $j \in \omega$ is such that $\tau_{j}=\varphi \uparrow n$. In order to justify this definition, we prove by induction that $\varphi \nmid n \in \prod_{i<n}[d(i)]^{\leq i+1}$ for all $n<\omega$. Suppose this holds for some $n . \varphi(n)$ is equal to some $\psi(j)$ such that $k_{n-1} \leq j<k_{n}$. But then $\varphi(n)=\psi(j) \subseteq d(n)$. Since we also have $|\varphi(n)|=|\psi(j)|<g(j)=\left|\tau_{j}\right|+1=n+1$, we conclude that $\varphi \uparrow(n+1) \in \prod_{i<n+1}[d(i)] \leq i+1$.

We already saw that $|\varphi(n)| \leq n+1$ for all $n \in \omega$, so $\varphi \in \mathcal{S}$. $\varphi$ is in $V$ since it is defined by means of $\psi$ and $\tau$, both belonging to $V$. Let us prove that $\varphi$ satisfies (ii). Let $n \in \omega$, and let $j \in \omega$ be such that $\tau_{j}=\varphi\lceil n$. Then $\pi\left(\varphi\lceil n)=\pi\left(\tau_{j}\right)=f(j) \in \psi(j)=\varphi(n)\right.$.

(ii) $\Rightarrow$ (i) Suppose (ii) holds and let $f \in{ }^{\omega} \omega$ be a function in $V[G]$. We define $\pi \in \mathcal{P}^{*}$ by $\pi(\tau)=f(|\tau|)$. Now let $\varphi$ be the slalom provided by (ii). Then $f(n)=\pi(\varphi \mid n) \in \varphi(n)$ holds for all $n \in \omega$, so $\varphi$ satisfies (i) as well. 
(ii) $\Rightarrow$ (iii) Assume (ii) and let $p \in B^{+}$and $\Sigma$ make a strategy for White in $\mathcal{G}_{\mathrm{sp}}$. We remind the reader that for every countable maximal antichain $A$ in $p \downarrow$ an enumeration $A=\left\{p_{j}^{A}: j<\omega\right\}$ is fixed. For each $\tau \in$ $\prod_{i<n}[\omega]^{\leq i+1}$ we define by recursion on $i \leq n$ antichains $A_{i}$ as follows: $A_{i}=\Sigma\left(\left\langle A_{0}, \tau(0), \ldots, A_{i-1}, \tau(i-1)\right\rangle\right)$, and let $b_{\tau, k}=p_{k}^{A_{n}}$.

Now, since $\left\{b_{\tau, k}: k<\omega\right\}$ is a maximal antichain in $p \downarrow$ for every $\tau$, $\pi=\left\{\left\langle\langle\tau, k\rangle, b_{\tau, k}\right\rangle: \tau \in \bigcup_{n<\omega} \prod_{i<n}[\omega] \leq i+1, k \in \omega\right\}$ is a nice name for a predictor in $V[G]$. Now, (ii) states that (in $V$ )

$$
\forall \pi \in \mathrm{N}_{\mathrm{n}}\left(\mathcal{P}^{*}\right) \forall p \in B^{+} \exists q \leq p \exists \varphi \in \mathcal{S} \forall n \in \omega q \Vdash \pi(\check{\varphi}\lceil\check{n}) \in \check{\varphi}(\check{n}) .
$$

Hence there are $q \leq p$ and $\varphi \in \mathcal{S}$ such that for all $n \in \omega \| \pi(\check{\varphi}\lceil\check{n}) \in$ $\check{\varphi}(\check{n}) \| \geq q$, i.e. $\bigvee_{k \in \varphi(n)} b_{\varphi \mid n, k}=\bigvee_{k \in \varphi(n)} \| \pi(\check{\varphi}\lceil\check{n})=\check{k} \| \geq q$. It follows that

$$
\bigwedge_{n \in \omega} \bigvee_{k \in \varphi(n)} b_{\varphi \mid n, k} \geq q
$$

which means that $\langle\varphi(n): n \in \omega\rangle$ is a sequence of Black's moves which ensures that, if White follows $\Sigma$, then Black wins. Thus, White does not have a winning strategy in $\mathcal{G}_{\text {sp }}$ played on $\mathbb{B}$.

(iii) $\Rightarrow$ (ii) Suppose (iii) holds. Let $p \in B^{+}$and let $\pi \in N_{n}\left(\mathcal{P}^{*}\right)$. We define a function $w: \bigcup_{n<\omega} \prod_{k<n}[\omega] \leq k+1 \rightarrow \mathcal{A}_{p}(\leq \omega)$ : for $\tau \in \bigcup_{n<\omega} \prod_{i<n}[\omega] \leq i+1$, let $w(\tau)=\{\|\pi(\check{\tau})=\breve{k}\| \wedge p: k<\omega\}$. Since $\pi$ is a nice name, $w(\tau)$ is a partition of $p$ for each $\tau$. Without loss of generality we can assume that $p_{k}^{w(\tau)}=\|\pi(\check{\tau})=\check{k}\| \wedge p$ (i.e. this is the $k$-th element of the enumeration fixed for $w(\tau))$. Since a winning strategy for White does not exist, by Theorem 2.59 there is a slalom $\varphi$ such that for some $q>0$

$$
\begin{aligned}
\bigwedge_{n<\omega} \| \pi(\check{\varphi}\lceil\check{n}) \in \check{\varphi}(\check{n}) \| \wedge p & =\bigwedge_{n<\omega} \bigvee_{k \in \varphi(n)}(\| \pi(\check{\varphi}\lceil\check{n})=k \| \wedge p) \\
& =\bigwedge_{n<\omega} \bigvee_{k \in \varphi(n)} p_{k}^{w(\varphi\lceil n)}=q .
\end{aligned}
$$

This proves (2.20), so (ii) holds.

The following two games are similar to $\mathcal{G}_{\mathrm{sp}}$, and they characterize the property of being $\omega^{\omega}$-bounding and the Laver property.

Definition 2.62 $\mathcal{G}_{\mathrm{b}}$ is the cut-and-choose game of type $(\omega, \omega, \omega)$, such that White wins the play $\left\langle p, A_{0}, a_{0}, \ldots, A_{n}, a_{n}, \ldots\right\rangle$ iff $\bigwedge_{n<\omega} \bigvee_{j \in a_{n}} p_{j}^{A_{n}}=0$. 
Theorem 2.63 For every complete Boolean algebra $\mathbb{B}$ the following conditions are equivalent:

(i) $\mathbb{B}$ is $\omega^{\omega}$-bounding, i.e. in every generic extension $V[G]$, for every $f \in \omega^{\omega} \omega$ there is $g \in\left({ }^{\omega} \omega\right)^{V}$ such that $f(n) \leq g(n)$ for all $n<\omega$;

(ii) in every generic extension $V[G]$, for every predictor $\pi \in \mathcal{P}$ there is $g \in\left({ }^{\omega} \omega\right)^{V}$ such that $\pi(g\lceil n) \leq g(n)$ for all $n<\omega ;$

(iii) White does not have a winning strategy in $\mathcal{G}_{\mathrm{b}}$ played on $\mathbb{B}$.

Proof. (i) $\Rightarrow$ (ii) Suppose that (i) holds; let $V[G]$ be a generic extension and $\pi \in \mathcal{P}^{V[G]}$. In $V$, let $\left\{\sigma_{j}: j<\omega\right\}$ be an arbitrary enumeration of the set $<\omega \omega$. Furthermore, let $h: \omega \rightarrow \omega$ be a function (in $V[G]$ ) given by $h(j)=\pi\left(\sigma_{j}\right)$. Since $\mathbb{B}$ is $\omega^{\omega}$-bounding, there is $h^{\prime}: \omega \rightarrow \omega$ in $V$ such that $h^{\prime}(j) \geq h(j)$ for all $j \in \omega$. Using recursion on $n$ we define $d(n)=\max \left\{h^{\prime}(j)\right.$ : $\left.\sigma_{j} \in \prod_{i<n} d(i)\right\}+1$. Then we fix an enumeration $\tau=\left\{\tau_{j}: j<\omega\right\}$ of the set $\bigcup_{n<\omega} \prod_{i<n} d(i)$ such that $i<j \Rightarrow\left|\tau_{i}\right| \leq\left|\tau_{j}\right|$.

Now let $f(j)=\pi\left(\tau_{j}\right)$, and let $f^{\prime} \in\left(\omega_{\omega} \omega\right)^{V}$ be such that $f(j) \leq f^{\prime}(j)$ holds for all $j \in \omega$. Since $f(j)=\pi\left(\tau_{j}\right)=\pi\left(\sigma_{k}\right)$ for some $k \in \omega$, and $\pi\left(\sigma_{k}\right)=h(k) \leq h^{\prime}(k)<d\left(\left|\sigma_{k}\right|\right)=d\left(\left|\tau_{j}\right|\right)$, we can assume that $f^{\prime}(j)<d\left(\left|\tau_{j}\right|\right)$. We define $g$ by recursion: $g(n)=f^{\prime}(j)$, where $j \in \omega$ is such that $\tau_{j}=g\lceil n$. Again, as in the proof of Theorem 2.61, we can prove that $g\left\lceil n \in \prod_{i<n} d(i)\right.$ for all $n<\omega$, which justifies the definition of $g$.

$g$ is in $V$ and satisfies (ii): if $n \in \omega$, let $j \in \omega$ be such that $\tau_{j}=g\lceil n$; then $\pi\left(g\lceil n)=\pi\left(\tau_{j}\right)=f(j) \leq f^{\prime}(j)=g(n)\right.$.

(ii) $\Rightarrow$ (iii) Assume (ii) and let $p \in B^{+}$and $\Sigma$ make a strategy for White in $\mathcal{G}_{\mathrm{b}}$. Let $\tau \in \prod_{i<n}[\omega] \leq i+1$. For $i \leq n$ we define by recursion: for $i=0$ let $A_{0}=\Sigma(\emptyset)$, and for $i>0$ let $A_{i}=\Sigma\left(\left\langle A_{0}, \tau(0), \ldots, A_{i-1}, \tau(i-1)\right\rangle\right)$. We also denote $b_{\tau, k}=p_{k}^{A_{n}}$. Again, $\pi=\left\{\left\langle\langle\tau, k\rangle, b_{\tau, k}\right\rangle: \tau \in \bigcup_{n<\omega} \prod_{i<n}[\omega] \leq i+1, k \in \omega\right\}$ is a nice name for a predictor in $V[G]$. By (ii) in $V$ holds

$$
\forall \pi \in \mathrm{N}_{\mathrm{n}}\left(\mathcal{P}^{*}\right) \forall p \in B^{+} \exists q \leq p \exists g \in{ }^{\omega} \omega \forall n \in \omega q \Vdash \pi(\check{g}\lceil\check{n}) \leq \check{g}(\check{n}) .
$$

Hence, $\bigvee_{k \leq g(n)} b_{g \nmid n, k}=\bigvee_{k \leq g(n)} \| \pi(\check{g}\lceil n)=k \| \geq q$, which implies that $\bigwedge_{n \in \omega} \bigvee_{k \leq g(n)} b_{g \nmid n, k} \geq q$. This means that $\langle g(n): n \in \omega\rangle$ is a sequence of Black's moves which ensures that Black wins if White follows $\Sigma$. Thus, White does not have a winning strategy in $\mathcal{G}_{\mathrm{b}}$ played on $\mathbb{B}$.

The rest of the proof (implications (ii) $\Rightarrow$ (i) and (iii) $\Rightarrow$ (ii)) is completely analogous to the proof of Theorem 2.61. 
Definition 2.64 $\mathcal{G}_{\text {lp }}$ is the cut-and-choose game of type $(\omega,<\omega, \mu)$, where $\mu(n)=n+1$ for $n \in \omega$, such that White wins the play $\left\langle p, A_{0}, a_{0}, \ldots, A_{n}\right.$, $\left.a_{n}, \ldots\right\rangle$ iff $\bigwedge_{n<\omega} \bigvee_{j \in a_{n}} p_{j}^{A_{n}}=0$.

Denoting $\mathcal{A}_{p}(<\omega)=\bigcup_{n \in \omega} \mathcal{A}_{p}(n)$ and applying Theorem 2.20 once more we get

Theorem 2.65 For every complete Boolean algebra $\mathbb{B}$ the following conditions are equivalent:

(a) White has a winning strategy in the game $\mathcal{G}_{\text {lp }}$ on $\mathbb{B}$;

(b) there are $p \in B^{+}$and $w: \bigcup_{n<\omega} \prod_{k<n}[\omega] \leq k+1 \rightarrow \mathcal{A}_{p}(<\omega)$ such that

$$
\forall \varphi \in \mathcal{S} \bigwedge_{k<\omega} \bigvee_{j \in \varphi(k)} p_{j}^{w(\varphi \mid k)}=0
$$

Theorem 2.66 For every complete Boolean algebra $\mathbb{B}$ the following conditions are equivalent:

(i) $\mathbb{B}$ has the Laver property, i.e. in every generic extension $V[G]$, for every $f \in{ }^{\omega} \omega$ for which there is $f^{\prime} \in\left({ }^{\omega} \omega\right)^{V}$ such that $f(n)<f^{\prime}(n)$ for all $n \in \omega$, there is $\varphi \in \mathcal{S}^{V}$ such that $f(n) \in \varphi(n)$ for all $n<\omega$;

(ii) in every generic extension $V[G]$, for every predictor $\pi \in \mathcal{P}^{*}$ for which there is $\theta \in\left(\mathcal{P}^{*}\right)^{V}$ such that $\pi(\tau)<\theta(\tau)$ for all $\tau \in \operatorname{dom} \pi$, there is $\varphi \in \mathcal{S}^{V}$ such that $\pi(\varphi\lceil n) \in \varphi(n)$ for all $n<\omega$;

(iii) White does not have a winning strategy in $\mathcal{G}_{\text {lp }}$ played on $\mathbb{B}$.

Proof. (i) $\Rightarrow$ (ii) Suppose that (i) holds; let $V[G]$ be a generic extension, $\pi \in$ $\left(\mathcal{P}^{*}\right)^{V[G]}$ and $\theta \in\left(\mathcal{P}^{*}\right)^{V}$ such that $\pi(\sigma)<\theta(\sigma)$ for all $\sigma \in$ dom $\pi$. Using recursion on $n$ we define $d(n)=\max \left\{\theta(\sigma): \sigma \in \prod_{i<n}[d(i)]^{\leq i+1}\right\}+1$. As in the proofs of the previous two theorems we denote $k_{m}=\left|\bigcup_{n<m} \prod_{i<n}[d(i)]^{\leq i+1}\right|$ and obtain an enumeration (in $V) \tau=\left\{\tau_{j}: j<\omega\right\}$ of $\bigcup_{n<\omega} \prod_{i<n}[d(i)] \leq i+1$ such that $i<j \Rightarrow\left|\tau_{i}\right| \leq\left|\tau_{j}\right|$.

Now we define $g(n)=\left|\tau_{n}\right|+1, f(n)=\pi\left(\tau_{n}\right)$ and $h(n)=\theta\left(\tau_{n}\right)$ for $n \in \omega$. Obviously, $h$ is in $V$ and $f(j)<h(j)$ for all $n \in \omega$. Let $\psi \in\left(\mathcal{S}^{g}\right)^{V}$ be such that $f(n) \in \psi(n)$ holds for all $n<\omega$, provided by (i) and Lemma 1.75.

Since $f(j)=\pi\left(\tau_{j}\right)<\theta\left(\tau_{j}\right)<d\left(\left|\tau_{j}\right|\right)$, we can assume that $\psi(j) \subseteq d\left(\left|\tau_{j}\right|\right)$. We define $\varphi$ by recursion: $\varphi(n)=\psi(j)$, where $j \in \omega$ is such that $\tau_{j}=\varphi\lceil n$. We have $\varphi \in \mathcal{S}^{V}$; let us prove that $\varphi$ satisfies (ii). Let $n \in \omega$, and let $j \in \omega$ be such that $\tau_{j}=\varphi\left\lceil n\right.$. Then $\pi\left(\varphi\lceil n)=\pi\left(\tau_{j}\right)=f(j) \in \psi(j)=\varphi(n)\right.$. 
(ii) $\Rightarrow$ (iii) Assume (ii) and let $p \in B^{+}$and $\Sigma$ make a strategy for White in $\mathcal{G}_{\text {lp }}$. Let $\tau \in \prod_{i<n}[\omega]^{\leq i+1}$. For $i \leq n$ we define, by recursion, $A_{i}=$ $\Sigma\left(\left\langle A_{0}, \tau(0), \ldots, A_{i-1}, \tau(i-1)\right\rangle\right.$, and let $b_{\tau, k}=p_{k}^{A_{n}}$. The antichain $A_{n}$ is finite so we can define $\theta(\tau)=\left|A_{n}\right|$. To simplify the notation, we can assume that $p_{k}^{A_{n}}=0$ for $k \geq\left|A_{n}\right|$. Again, $\pi=\left\{\left\langle\langle\tau, k\rangle, b_{\tau, k}\right\rangle: \tau \in\right.$ $\left.\bigcup_{n<\omega} \prod_{i<n}[\omega] \leq i+1, k \in \omega\right\}$ is a nice name for a predictor in $V[G]$, and we have $\left.1 \Vdash \forall \tau \in\left(\prod_{i<n}[\omega]\right]^{\leq i+1}\right)^{\sim} \pi(\tau) \leq \check{\theta}(\tau)$. Now, (ii) claims that

$$
\begin{aligned}
& \forall \pi \in \mathrm{N}_{\mathrm{n}}\left(\mathcal{P}^{*}\right) \forall \theta \in \mathcal{P}^{*} \forall p \in B^{+}\left(\forall \tau \in \prod_{i<n}[\omega]^{\leq i+1} p \Vdash \pi(\tau) \leq \theta(\tau) \Rightarrow\right. \\
& \exists q \leq p \exists \varphi \in \mathcal{S} \forall n \in \omega q \Vdash \pi(\check{\varphi}\lceil\check{n}) \in \check{\varphi}(\check{n}))
\end{aligned}
$$

so we can find $q \leq p$ and $\varphi \in \mathcal{S}$ such that for all $n \in \omega \| \pi(\check{\varphi}\lceil\check{n}) \in$ $\check{\varphi}(\check{n}) \| \geq q$, i.e. $\bigvee_{k \in \varphi(n)} b_{\varphi \mid n, k}=\bigvee_{k \in \varphi(n)} \| \pi(\check{\varphi}\lceil\check{n})=\check{k} \| \geq q$. It follows that $\bigwedge_{n \in \omega} \bigvee_{k \in \varphi(n)} b_{\varphi \mid n, k} \geq q$ which means that $\langle\varphi(n): n \in \omega\rangle$ is a sequence of Black's moves which ensures that, if White follows $\Sigma$, then Black wins. Thus, White does not have a winning strategy in $\mathcal{G}_{\text {lp }}$ played on $\mathbb{B}$.

(iii) $\Rightarrow$ (ii) Suppose (iii) holds and let $p \in B^{+}$. Let $\pi$ be a name for a set in $V[G]$ such that $1 \Vdash \pi \in \overline{\mathcal{P}^{*}}$ and let $\theta \in \mathcal{P}^{*}$ be such that for $\tau \in \prod_{i<n}[\omega] \leq i+1$ $p \Vdash \pi(\check{\tau}) \leq \check{\theta}(\check{\tau})$, i.e. $p \wedge\|\pi(\check{\tau}) \leq \check{\theta}(\check{\tau})\|=p$. We define a function $w$ : $\bigcup_{n<\omega} \prod_{i<n}[\omega] \leq i+1 \rightarrow \mathcal{A}_{p}(<\omega): w(\tau)=\{\|\pi(\check{\tau})=\check{k}\| \wedge p: k \in \theta(\tau)\}$. Clearly, $w(\tau)$ is an antichain in $p \downarrow$ of cardinality at most $\theta(\tau)$ for each $\tau$; it is maximal because $\bigvee_{k<\theta(\tau)}\|\pi(\check{\tau})=\check{k}\| \wedge p=p$. Without loss of generality we can assume that $p_{k}^{w(\tau)}=\|\pi(\check{\tau})=\check{k}\| \wedge p$. By Theorem 2.65 there is $\varphi \in \mathcal{S}$ such that $\bigwedge_{n<\omega} \bigvee_{k \in \varphi(n)} p_{k}^{w(\varphi \mid n)}>0$. But $\bigvee_{k \in \varphi(n)} p_{k}^{w(\varphi \mid n)}=\| \pi(\check{\varphi}\lceil\check{n}) \in \check{\varphi}(\check{n}) \|$, so there is $q \leq p$ such that $\bigwedge_{n<\omega} \| \pi(\check{\varphi}\lceil\check{n}) \in \check{\varphi}(\check{n}) \| \geq q$. We proved (2.23), so (ii) holds.

The rest of the proof (implications (ii) $\Rightarrow(\mathrm{i})$ and (iii) $\Rightarrow(\mathrm{ii})$ ) is completely analogous to the proof of Theorem 2.61. 


\section{Chapter 3}

\section{A power collapsing game}

We define another game, first introduced in [25] (for $\kappa=\omega$ ), and further investigated in [26].

Definition 3.1 The game $\mathcal{G}_{\mathrm{ls}}(\kappa)$ is the cut-and-choose game of type $(\kappa, 2,2)$ such that White wins the play $\left\langle p, p_{0}, i_{0}, \ldots, p_{\alpha}, i_{\alpha}, \ldots\right\rangle$ if and only if

$$
\bigwedge_{\beta \in \kappa} \bigvee_{\alpha \geq \beta} p_{\alpha}^{i_{\alpha}}=0
$$

(For $\kappa=\omega$ the left-hand side of the equation above is called "the limit superior" of the sequence $\left\langle p_{\alpha}^{i_{\alpha}}: \alpha<\omega\right\rangle$ and denoted $\lim \sup _{\alpha<\omega} p_{\alpha}^{i_{\alpha}}$; hence the notation $\mathcal{G}_{\text {ls }}$.)

First we compare $\mathcal{G}_{\text {ls }}(\kappa)$ to $\mathcal{G}_{\text {dist }}(\kappa, 2,2)$.

Lemma 3.2 Let $\kappa \geq \omega$ be a cardinal, $\mathbb{B}$ a complete Boolean algebra, $\left\langle b_{\alpha}\right.$ : $\alpha<\kappa\rangle$ a $\kappa$-sequence in $\mathbb{B}$ and $\sigma=\left\{\left\langle\check{\alpha}, b_{\alpha}\right\rangle: \alpha \in \kappa\right\}$ the corresponding nice name for a subset of $\kappa$. Then

(a) $\bigwedge_{\alpha \in \kappa} b_{\alpha}=\|\sigma=\check{\kappa}\|$.

(b) $\bigwedge_{\beta \in \kappa} \bigvee_{\alpha \geq \beta} b_{\alpha}=\| \sigma$ is unbounded in $\check{\kappa} \|$.

(c) $\bigwedge_{\alpha \in \kappa} b_{\alpha} \leq \bigwedge_{\beta \in \kappa} \bigvee_{\alpha \geq \beta} b_{\alpha}$.

Proof. (a) $\bigwedge_{\alpha \in \kappa} b_{\alpha}=\bigwedge_{\alpha \in \kappa}\|\check{\alpha} \in \sigma\|=\|\forall \alpha \in \check{\kappa} \alpha \in \sigma\|=\|\sigma=\check{\kappa}\|$.

(b) $\bigwedge_{\beta \in \kappa} \bigvee_{\alpha \geq \beta} b_{\alpha}=\|\forall \beta \in \check{\kappa} \exists \alpha \geq \beta \alpha \in \sigma\|=\| \sigma$ is unbounded in $\check{\kappa} \|$.

(c) Obvious.

Using Lemma 3.2 it is easy to prove 
Lemma 3.3 For every cardinal $\kappa \geq \omega$ and every complete Boolean algebra $\mathbb{B}$ :

(a) If White has a winning strategy in $\mathcal{G}_{\mathrm{ls}}(\kappa)$, then White has a winning strategy in $\mathcal{G}_{\text {dist }}(\kappa, 2,2)$ as well.

(b) If Black has a winning strategy in $\mathcal{G}_{\text {dist }}(\kappa, 2,2)$, then Black has a winning strategy in $\mathcal{G}_{\mathrm{ls}}(\kappa)$ as well.

\subsection{The game $\mathcal{G}_{\mathrm{ls}}(\kappa)$ from White's point of view}

Theorem 3.4 For every infinite cardinal $\kappa$ and every complete Boolean algebra $\mathbb{B}$ the following conditions are equivalent:

(a) White has a winning strategy in the game $\mathcal{G}_{\text {ls }}(\kappa)$ on $\mathbb{B}$;

(b) there are $p \in B^{+}$and $w:<\kappa 2 \rightarrow(0, p)_{\mathbb{B}}$ such that

$$
\forall i: \kappa \rightarrow 2 \bigwedge_{\beta<\kappa} \bigvee_{\alpha \geq \beta} w_{i \uparrow \alpha}^{i(\alpha)}=0
$$

(c) there are $p \in B^{+}$and $w:{ }^{<\kappa} 2 \rightarrow[0, p]_{\mathbb{B}}$ such that (3.1) holds;

(d) there are $p \in B^{+}$and $\rho \in V^{\mathbb{B}}$ such that

$$
\begin{aligned}
& p \Vdash \rho \subseteq\left(\left({ }^{<\kappa} 2\right)^{V}\right)^{\sim} \wedge \forall \varphi \in\left((<\kappa 2)^{V}\right)^{\sim}\left(\varphi^{\frown} \check{0} \in \rho \dot{V} \varphi^{-\check{1}} \in \rho\right) \\
& \wedge \forall i \in\left(\left({ }^{\kappa} 2\right)^{V}\right)^{\sim} \exists \beta \in \check{\kappa} \forall \alpha \geq \beta \quad i\lceil\alpha \notin \rho \text {; }
\end{aligned}
$$

(e) in some generic extension, $V_{\mathbb{B}}[G]$, there is a subset $R$ of the tree $\left({ }^{<\kappa} 2\right)^{V}$, containing either $\varphi^{-} 0$ or $\varphi^{-1}$ for each $\varphi \in\left({ }^{<\kappa} 2\right)^{V}$ and such that for each $i \in\left({ }^{\kappa} 2\right)^{V}$ the set $\{\alpha \in \kappa: i\lceil\alpha \in R\}$ is bounded in $\kappa$.

Proof. $(\mathrm{a}) \Leftrightarrow(\mathrm{b}) \Leftrightarrow(\mathrm{c})$ follows from Theorem 2.21 .

(b) $\Rightarrow(\mathrm{d})$. In $V$, let $p \in B^{+}$and $w:<\kappa 2 \rightarrow(0, p)_{\mathbb{B}}$ be the objects provided by (b). We define $v_{\varphi} \in[0, p]_{\mathbb{B}}$ for $\varphi \in^{<\kappa} 2$, as follows. For $\varphi=\emptyset$ let $v_{\emptyset}=p$, for $\varphi \in{ }^{<\kappa} 2$ of limit height let $v_{\varphi}=0$ and for $\varphi \in{ }^{<\kappa} 2$ and $k \in 2$ let $v_{\varphi-k}=w_{\varphi}^{k}$. Then $\rho=\left\{\left\langle\breve{\varphi}, v_{\varphi}\right\rangle: \varphi \in{ }^{<\kappa} 2\right\}$ is a nice name for a subset of $\left({ }^{<\kappa} 2\right)^{V}$ and clearly

$$
1 \Vdash \forall \gamma \in(\kappa \cap \operatorname{Lim})^{\curvearrowright} \rho \cap\left(\left({ }^{\gamma} 2\right)^{V}\right)^{\curvearrowright}=\check{\emptyset} .
$$

Let $G$ be a $\mathbb{B}$-generic ultrafilter over $V$ containing $p$. If $\varphi \in\left({ }^{<\kappa} 2\right)^{V}$, then either $w_{\varphi}$ or $p \backslash w_{\varphi}$ belongs to $G$. Since $\left.w_{\varphi}=w_{\varphi}^{0}=v_{\varphi\urcorner 0}=\| \check{\varphi}\right\urcorner \tilde{0} \in \rho \|$ 
and $\left.p \backslash w_{\varphi}=w_{\varphi}^{1}=v_{\varphi\urcorner 1}=\| \check{\varphi}\right\urcorner \check{1} \in \rho \|$ we have that either $\varphi^{\frown} 0 \in \rho_{G}$ or $\varphi^{\wedge} 1 \in \rho_{G}$.

Let $i \in\left({ }^{\kappa} 2\right)^{V}$. Since $\left\|(i \uparrow(\alpha+1))^{\sim} \in \rho\right\|=v_{i \uparrow(\alpha+1)}=v_{(i \uparrow \alpha)-i(\alpha)}=w_{i\lceil\alpha}^{i(\alpha)}$, according to (3.1) we have $\|\forall \beta \in \check{\kappa} \exists \alpha \geq \beta \check{i} \uparrow(\alpha+1) \in \rho\|=0$, that is $1 \Vdash \exists \beta \in \check{\kappa} \forall \alpha \geq \beta \check{i} \uparrow(\alpha+1) \notin \rho$. This and (3.2) implies $1 \Vdash \exists \beta \in \check{\kappa} \forall \alpha \geq$ $\beta \check{i}\lceil\alpha \notin \rho$, so $(\mathrm{d})$ is true.

(d) $\Rightarrow\left(\right.$ c). Let $p \in B^{+}$and $\rho \in V^{\mathbb{B}}$ be the objects provided by (d). In $V$ for each $\varphi \in\left({ }^{<\kappa} 2\right)^{V}$ we define $w_{\varphi}=\left\|\left(\varphi^{\frown} 0\right)^{`} \in \rho\right\| \wedge p$ and verify (3.1). Let $i \in\left({ }^{\kappa} 2\right)^{V}$. By $(\mathrm{d}), p \Vdash\left(\left(i\lceil\alpha)^{\frown} 0\right)^{`} \in \rho \dot{\vee}\left(\left(i\lceil\alpha)^{\frown} 1\right)^{`} \in \rho\right.\right.$ holds for each $\alpha \in \kappa$, that is $p \leq a_{0} \vee a_{1}$ and $p \wedge a_{0} \wedge a_{1}=0$, where $a_{k}=\|\left(\left(i\lceil\alpha)^{\wedge} k\right)^{`} \in \rho \|\right.$, $k \in\{0,1\}$, which clearly implies $p \wedge a_{0}^{\prime}=p \wedge a_{1}$, that is

$$
p \wedge \|\left(( i \lceil \alpha ) ^ { \frown } 0 ) ^ { ` } \in \rho \| ^ { \prime } = p \wedge \| \left(\left(i\lceil\alpha)^{\frown} 1\right)^{`} \in \rho \| .\right.\right.
$$

Let us prove that for each $\alpha \in \kappa$

$$
w_{i\lceil\alpha}^{i(\alpha)}=\left\|(i \uparrow(\alpha+1))^{\swarrow} \in \rho\right\| \wedge p .
$$

If $i(\alpha)=0$, then $w_{i \uparrow \alpha}^{i(\alpha)}=w_{i \uparrow \alpha}=\left\|\left((i \uparrow \alpha)^{\frown} 0\right)^{\curlyvee} \in \rho\right\| \wedge p=\|\left(\left(i\lceil\alpha)^{`} i(\alpha)\right)^{`} \in\right.$ $\rho\|\wedge p=\|(i \uparrow(\alpha+1))^{`} \in \rho \| \wedge p$ and (3.4) holds. If $i(\alpha)=1$, then according to $(3.3), w_{i\lceil\alpha}^{i(\alpha)}=p \backslash w_{i\lceil\alpha}=p \wedge\left(\left\|\left(\left(i\lceil\alpha)^{\frown} 0\right)^{`} \in \rho \| \wedge p\right)^{\prime}=p \wedge\right\|\left(\left(i\lceil\alpha)^{\frown} 0\right)^{`} \in\right.\right.$ $\rho\left\|^{\prime}=p \wedge\right\|\left(\left(i\lceil\alpha)^{\frown} 1\right)^{\frown} \in \rho\|=p \wedge\|\left(\left(i\lceil\alpha)^{\frown} i(\alpha)\right)^{`} \in \rho \|\right.\right.$ and (3.4) holds again.

Now $\bigwedge_{\beta<\kappa} \bigvee_{\alpha \geq \beta} w_{i\lceil\alpha}^{i(\alpha)}=p \wedge\|\forall \beta<\check{\kappa} \exists \alpha \geq \beta \check{i} \uparrow(\alpha+1) \in \rho\|=0$, since by (d) $p \leq \| \exists \beta<\check{\kappa} \forall \alpha \geq \beta \check{i}\lceil\alpha \notin \rho \|$. Thus (c) is proved.

$(\mathrm{d}) \Rightarrow(\mathrm{e})$ is obvious and (e) $\Rightarrow(\mathrm{d})$ follows from The Maximum Principle (Theorem 1.36).

Now we turn to the main theorem of this section.

Theorem 3.5 Let $\kappa$ be an infinite cardinal and $\mathbb{B}$ a complete Boolean algebra. Then $(\mathrm{a}) \Rightarrow(\mathrm{b}) \Rightarrow(\mathrm{c})$, where

(a) In some generic extension, $V_{\mathbb{B}}[G], \kappa$ is a regular cardinal and the cardinal $\left(2^{\kappa}\right)^{V}$ is collapsed to $\kappa$;

(b) White has a winning strategy in the game $\mathcal{G}_{\mathrm{ls}}(\kappa)$ played on $\mathbb{B}$;

(c) in some generic extension, $V_{\mathbb{B}}[G]$, the sets $\left({ }^{\kappa} 2\right)^{V}$ and $\left({ }^{<\kappa} 2\right)^{V}$ are of the same size. 
Proof. (a) $\Rightarrow$ (b). Let $V[G] \models\left|\left(2^{\kappa}\right)^{V}\right|=\kappa \in$ Reg. We will prove that (e) of Theorem 3.4 holds. By the assumption, in $V[G]$ there is an enumeration $\left({ }^{\kappa} 2\right)^{V}=\left\{i_{\xi}: \xi<\kappa\right\}$. Clearly, for all $\xi<\kappa$ and all $\alpha<\kappa$ we have $i_{\xi}\left\lceil\alpha \in(<\kappa 2)^{V}\right.$. Moreover, $g^{i_{\xi}}=\left\{i_{\xi}\lceil\alpha: \alpha<\kappa\} \in V\right.$ and

$$
\bigcup_{\xi<\kappa} g^{i_{\xi}}=\left(\kappa^{<} 2\right)^{V} \text {. }
$$

In $V[G]$, for each $\xi<\kappa$ let $S_{\xi}=\left\{\varphi^{\wedge} k: \varphi \in g^{i_{\xi}} \wedge k \in 2\right\}$ and $T_{\xi}=$ $S_{\xi} \backslash \bigcup_{\zeta<\xi} S_{\zeta}$. Then $g^{i_{\xi}} \backslash \bigcup_{\gamma \in \kappa \cap \operatorname{Lim}}\left({ }^{\gamma} 2\right)^{V} \subseteq S_{\xi} \subseteq\left({ }^{<\kappa} 2\right)^{V} \backslash \bigcup_{\gamma \in \kappa \cap \operatorname{Lim}}\left({ }^{\gamma} 2\right)^{V}$ for each $\xi \in \kappa$, which together with (3.5) implies

$$
\bigcup_{\xi<\kappa} S_{\xi}=\bigcup_{\beta \in \kappa \backslash \operatorname{Lim}}\left({ }^{\beta} 2\right)^{V} .
$$

Clearly, $\bigcup_{\xi<\kappa} T_{\xi}=\bigcup_{\xi<\kappa} S_{\xi}$. In $V[G]$, for $\xi<\kappa$ let

$$
R_{\xi}=T_{\xi} \backslash g^{i_{\xi}}=S_{\xi} \backslash\left(\bigcup_{\zeta<\xi} S_{\zeta} \cup g^{i_{\xi}}\right) .
$$

We show that the set $R=\bigcup_{\xi<\kappa} R_{\xi}$ satisfies conditions of (e) of Theorem 3.4. First, since $R_{\xi} \subseteq S_{\xi}$ for all $\xi<\kappa$, we have $R \subseteq \bigcup_{\xi<\kappa} S_{\xi}$, so, by (3.6), $R \subseteq\left({ }^{<} 2\right)^{V}$ and the elements of $R$ are not of limit height.

Secondly, let $i \in\left({ }^{\kappa} 2\right)^{V}$. Then $i=i_{\xi}$ for some $\xi<\kappa$ and we prove

$$
\forall \zeta \geq \xi R_{\zeta} \cap g^{i_{\xi}}=\emptyset
$$

By definition, for $\zeta=\xi$ we have $R_{\zeta} \cap g^{i_{\xi}}=\emptyset$ and if $\zeta>\xi$, then $R_{\zeta} \cap S_{\xi}=\emptyset$. Thus, since the elements of $g^{i_{\xi}}$ of successor height are in $S_{\xi}$ and since $R_{\zeta}$ does not contain elements of limit height, it follows that $R_{\zeta} \cap g^{i_{\xi}}=\emptyset$.

According to (3.7) we have $R \cap g^{i_{\xi}}=\bigcup_{\zeta<\kappa} R_{\zeta} \cap g^{i_{\xi}}=\bigcup_{\zeta<\xi} R_{\zeta} \cap g^{i_{\xi}}$ which implies

$$
R \cap g^{i_{\xi}} \subseteq \bigcup_{\zeta<\xi} S_{\zeta} \cap g^{i_{\xi}}
$$

Now for $\zeta<\xi$, let $\beta_{\zeta}=\min \left\{\alpha \in \kappa: i_{\zeta}(\alpha) \neq i_{\xi}(\alpha)\right\}$. Then $i_{\xi}\left\lceil\alpha \notin S_{\zeta}\right.$ for $\alpha>\beta_{\zeta}+1$, so $S_{\zeta} \cap g^{i_{\xi}} \subseteq\left\{i_{\xi}\left\lceil\alpha: \alpha \leq \beta_{\zeta}+1\right\}\right.$. Thus, according to (3.8), $R \cap g^{i_{\xi}} \subseteq\left\{i_{\xi}\lceil\alpha: \alpha<\beta\}\right.$, where $\beta=\sup \left\{\beta_{\zeta}+1: \zeta<\xi\right\}+1$. Since $\kappa$ is a regular cardinal in $V[G]$, we have $\beta<\kappa$ and $\{\alpha \in \kappa: i \uparrow \alpha \in R\}$ is a bounded subset of $\kappa$.

Finally, for an arbitrary $\varphi \in\left({ }^{<\kappa} 2\right)^{V}$ we prove $\varphi^{\urcorner} 0 \in R \dot{\vee} \varphi^{\wedge} 1 \in R$. According to (3.5), there is $\xi=\min \left\{\zeta<\kappa: \varphi \in g^{i_{\zeta}}\right\}$. Then $\varphi \in g^{i_{\xi}}$, hence $\varphi^{\urcorner} 0, \varphi^{\wedge} 1 \in S_{\xi}$ and for $\zeta<\xi$ we have $\varphi^{\urcorner} 0, \varphi^{\urcorner} 1 \notin S_{\zeta}$, thus $\varphi^{\urcorner} 0, \varphi^{\wedge} 1 \in T_{\xi}$. Since $\varphi \in g^{i_{\xi}}$, either $\varphi^{\frown} 0$ or $\varphi^{\frown} 1$ belongs to $g^{i_{\xi}}$.

If $\varphi^{\frown} 0 \in g^{i_{\xi}}$, then $\varphi^{\frown} 0 \notin R_{\xi} \ni \varphi^{\frown} 1$, thus $\varphi^{\frown} 1 \in R$. Since $\varphi^{\frown} 0 \in T_{\xi}$ and $R \cap T_{\xi}=\bigcup_{\zeta<\kappa}\left(T_{\zeta} \backslash g^{i_{\zeta}}\right) \cap T_{\xi}=T_{\xi} \backslash g^{i_{\xi}}=R_{\xi}$, we have $\varphi^{\frown} 0 \notin R$. Similarly, $\varphi^{\wedge} 1 \in g^{i_{\xi}}$ implies $\varphi^{-} 0 \in R \not \supset \varphi^{\wedge} 1$. 
$(\mathrm{b}) \Rightarrow(\mathrm{c})$. Suppose White has a winning strategy in the game $\mathcal{G}_{\mathrm{ls}}(\kappa)$ played on $\mathbb{B}$. Let $p \in B^{+}$and $\rho \in V^{\mathbb{B}}$ be the objects provided by (d) of Theorem 3.4. We prove that

$$
p \Vdash\left|\left(\left(2^{\kappa}\right)^{V}\right)^{\smile}\right|=\left|\left((<\kappa 2)^{V}\right)^{\smile}\right| .
$$

Let $G$ be a $\mathbb{B}$-generic filter over $V$ such that $p \in G$. In $V[G]$, for $\beta \in \kappa$ let

$$
S_{\beta}=\left\{i \in\left({ }^{\kappa} 2\right)^{V}: \forall \alpha \geq \beta \quad i\left\lceil\alpha \notin \rho_{G}\right\} .\right.
$$

If $i \in\left({ }^{\kappa} 2\right)^{V}$, then, according to (d) of Theorem 3.4, there is $\beta<\kappa$ such that $i \uparrow \alpha \notin \rho_{G}$ for all $\alpha \geq \beta$, which implies $i \in S_{\beta}$. Thus

$$
\left({ }^{\kappa} 2\right)^{V}=\bigcup_{\beta<\kappa} S_{\beta} .
$$

If $i \in S_{\beta}$, then $i \in V$ and, hence, $i\left\lceil\beta \in\left({ }^{3} 2\right)^{V}\right.$. Consequently, the function $f: S_{\beta} \rightarrow\left({ }^{\beta} 2\right)^{V}$ given by $f(i)=i \uparrow \beta$ is well-defined and we prove $f$ is an injection. Let $i, j \in S_{\beta}$, where $i \neq j$ and suppose $i \uparrow \beta=j \uparrow \beta$. Then $\alpha_{0}=\min \{\alpha \in \kappa: i(\alpha) \neq j(\alpha)\} \geq \beta$. According to (d) of Theorem 3.4, for $\varphi=i\left\lceil\alpha_{0}=j\left\lceil\alpha_{0}\right.\right.$ we have $\varphi^{\urcorner} 0 \in \rho_{G} \dot{\vee} \varphi^{\urcorner} 1 \in \rho_{G}$ and, since $i\left(\alpha_{0}\right) \neq j\left(\alpha_{0}\right)$, it follows that $i \uparrow\left(\alpha_{0}+1\right) \in \rho_{G}$ or $j \uparrow\left(\alpha_{0}+1\right) \in \rho_{G}$ which is impossible because $i, j \in S_{\beta}$ and $\alpha_{0}+1>\beta$. Thus $f$ is an injection and, hence, $\left|S_{\beta}\right|^{V[G]} \leq\left|\left({ }^{\beta} 2\right)^{V}\right|^{V[G]} \leq\left|\left({ }^{<\kappa} 2\right)^{V}\right|^{V[G]}$. Now (3.10) implies $\left|\left(2^{\kappa}\right)^{V}\right|^{V[G]} \leq$ $|\kappa|^{V[G]}\left|\left({ }^{<\kappa} 2\right)^{V}\right|^{V[G]}=\left|\left({ }^{<\kappa} 2\right)^{V}\right|^{V[G]}$ and (3.9) is proved.

Theorem 3.6 Let $\kappa \geq \omega$ be a regular cardinal satisfying $2^{<\kappa}=\kappa$ and $\mathbb{B}$ a complete Boolean algebra such that $1 \Vdash_{\mathbb{B}} \check{\kappa} \in$ Reg. Then White has a winning strategy in the game $\mathcal{G}_{\mathrm{ls}}(\kappa)$ played on $\mathbb{B}$ iff in some generic extension the cardinal $\left(2^{\kappa}\right)^{V}$ is collapsed to $\kappa$.

For $\kappa=\omega$ we get the main result of [25].

Corollary 3.7 White has a winning strategy in the game $\mathcal{G}_{\mathrm{ls}}(\omega)$ played on a complete Boolean algebra $\mathbb{B}$ iff in some generic extension $\mathfrak{c}^{V}$ is collapsed to $\omega$.

We will prove in Theorem 3.21 that $\mathfrak{h}_{2}(\mathbb{B})$ is the minimal cardinal $\kappa$ such that White can have a winning strategy in the game $\mathcal{G}_{\mathrm{ls}}(\kappa)$. In Theorem 3.11 below we give an analogue of Corollary 3.7 for $\mathfrak{h}_{2}(\mathbb{B})$. To do this we need the following three statements.

Lemma 3.8 If in some generic extension $V[G]$ a cardinal $\kappa$ is collapsed to $\lambda$, then $\lambda$ obtains a new subset in $V[G]$. 
Proof. In $V$, let $X \subseteq P(\lambda)$ where $|X|=\lambda^{+}$and let $f: \lambda^{+} \rightarrow X$ be a bijection. Since $\left|\left(\lambda^{+}\right)^{V}\right|^{V[G]}=\lambda$, in $V[G]$ there is a bijection $\varphi: \lambda \rightarrow \lambda^{+}$. Suppose that the set $Y=\bigcup_{\alpha \in \lambda}(\{\alpha\} \times f(\varphi(\alpha))) \subseteq \lambda \times \lambda$ belongs to $V$. Since for each $\alpha<\lambda$ we have $\varphi(\alpha)=f^{-1}\left(\pi_{2}[Y \cap(\{\alpha\} \times \lambda)]\right.$ ) (where $\pi_{2}: \lambda \times \lambda \rightarrow \lambda$ is defined by $\pi_{2}(\langle\alpha, \beta\rangle)=\beta$ ) and since $f, \pi_{2}, Y \in V$, we would have $\varphi \in V$, which is impossible. Thus the set $\lambda \times \lambda$ obtains a new subset $Y$ in $V[G]$. But then $\lambda$ obtains one too: if $g: \lambda \times \lambda \rightarrow \lambda$ is a bijection in $V, g[Y]$ can not be in $V$, since $Y=g^{-1}[g[Y]]$ would then be in $V$ too.

Lemma 3.9 Suppose $0^{\sharp}$ does not exist and let $V[G]$ be a generic extension. Then

(a) For each $X \in V[G]$ satisfying $X \subseteq V$ there is $A \in V$ such that $X \subseteq A$ and

$$
|X|^{V[G]} \leq|A|^{V[G]} \leq|X|^{V[G]}+\omega_{1}^{V[G]}
$$

(b) If $\kappa$ is a regular cardinal and $\mathrm{cf}^{V[G]}(\kappa)=\lambda<\kappa$, then

$$
|\kappa|^{V[G]}=\lambda \vee\left(\lambda=\omega \wedge|\kappa|^{V[G]}=\omega_{1}^{V[G]}<\kappa\right) .
$$

Consequently, $\kappa$ is collapsed in $V[G]$.

Proof. (a) In $V[G]$, let $\xi=\sup \{\operatorname{rank}(x): x \in X\}$. Since rank is an absolute function we have $X \subseteq\left(V_{\xi+1}\right)^{V}$. Let $\left|\left(V_{\xi+1}\right)^{V}\right|^{V}=\kappa$ and let $f$ : $V_{\xi+1} \rightarrow \kappa$ be a bijection belonging to $V$. Then $f[X]$ is a set of ordinals in $V[G]$ and, since $0^{\sharp} \notin V[G]$ as well, applying Jensen's Covering Theorem 1.8 in $V[G]$ and taking into account that the constructible universe is also absolute (so $L^{V[G]}=L^{V}=L$ ), we obtain $C \in L$ such that $f[X] \subseteq C$ and $|C|^{V[G]}=|f[X]|^{V[G]}+\omega_{1}^{V[G]}$. Now $A=f^{-1}[C \cap \kappa] \in V, X \subseteq A$ and, since $|A|^{V[G]} \leq|C|^{V[G]}$ we have (3.11).

(b) Let $f: \lambda \rightarrow \kappa$ be a cofinal increasing function belonging to $V[G]$. Then $X=f[\lambda] \subseteq \kappa$ and $|X|^{V[G]}=\lambda$ so, by (a), there is $A \in V$ such that $X \subseteq A$ and $\lambda \leq|A|^{V[G]} \leq \lambda+\omega_{1}^{V[G]}$. Without loss of generality we can suppose $A \subseteq \kappa$. Now, since $A$ is unbounded in $\kappa$, we have $|A|^{V}=\kappa$, which implies $|A|^{V[G]}=|\kappa|^{V[G]}$ and, hence,

$$
\lambda \leq|\kappa|^{V[G]} \leq \lambda+\omega_{1}^{V[G]} .
$$

If $|\kappa|^{V[G]}=\lambda$, then (3.12) is proved. Otherwise $\lambda<\omega_{1}^{V[G]}$, which together with the fact that $\lambda$ is a regular cardinal in $V[G]$ implies $\lambda=\omega$. Now (3.13) implies $|\kappa|^{V[G]}=\omega_{1}^{V[G]}$ and, since $\operatorname{cf}^{V[G]}(\kappa)=\omega$ we have $\kappa \neq \omega_{1}^{V[G]}$. Thus $\kappa>\omega_{1}^{V[G]}$ and (3.12) holds again. 
Lemma 3.10 Suppose $0^{\sharp}$ does not exist. If $\mathbb{B}$ is a atomless complete Boolean algebra and $\kappa_{0}=\mathfrak{h}_{2}(\mathbb{B})$, then

(a) forcing by $\mathbb{B}$ preserves cardinals $\leq \kappa_{0}$;

(b) $\kappa_{0}$ is a regular cardinal in $V$;

(c) forcing by $\mathbb{B}$ preserves cofinalities $\leq \kappa_{0}$;

(d) forcing by $\mathbb{B}$ preserves the regularity of $\kappa_{0}$.

Proof. (a) Suppose that $\lambda<\kappa \leq \kappa_{0}$ and that in some generic extension $V[G]$ by $\mathbb{B} \kappa$ is collapsed to $\lambda$. Then, according to Lemma $3.8, P^{V[G]}(\lambda) \neq$ $P^{V}(\lambda)$, which is impossible by Theorem 1.59 because the algebra $\mathbb{B}$ is $(\lambda, 2)$ distributive.

(b) The case $\kappa_{0}=\omega$ is trivial, so we can assume $\kappa_{0} \geq \omega_{1}$. Suppose that $\operatorname{cf}^{V}\left(\kappa_{0}\right)=\lambda<\kappa_{0}$ and let $f: \lambda \rightarrow \kappa_{0}$ be a cofinal increasing function. $\mathbb{B}$ is not $\left(\kappa_{0}, 2\right)$-distributive, so by Theorem 1.59 there is a set $X \subseteq \kappa_{0}$ in some extension $V[G]$ by $\mathbb{B}$ such that $X \notin V$. Since $\kappa_{0}$ is a limit cardinal and $P^{V[G]}(\kappa)=P^{V}(\kappa)$ for each $\kappa<\kappa_{0}$ we have $Y=\{X \cap f(\alpha): \alpha<\lambda\} \subset V$ so, by Lemma 3.9 (a), there is $A \in V$ such that $Y \subseteq A$ and $|A|^{V[G]} \leq$ $|Y|^{V[G]}+\omega_{1}^{V[G]}$. According to (a) we have $\omega_{1}^{V[G]}=\omega_{1}^{V}$ and $|Y|^{V[G]} \leq \lambda$ which implies $|A|^{V[G]}<\kappa_{0}$. Since $|A|^{V} \geq \kappa_{0}$ would imply that $\kappa_{0}$ is collapsed which is not the case by (a), we have $|A|^{V}<\kappa_{0}$ as well. Clearly $X=\bigcup Y$ and, hence, $Y \notin V$. Thus the set $A$ and, consequently, the cardinal $|A|^{V}<\kappa_{0}$ obtains a new subset in $V[G]$. Hence $\mathbb{B}$ is not $\left(|A|^{V}, 2\right)$-distributive, which is impossible by the minimality of $\kappa_{0}$.

(c) According to Lemma 1.33 it is sufficient to prove that each regular cardinal $\kappa \leq \kappa_{0}$ remains regular in every extension $V[G]$ by $\mathbb{B}$. Suppose $\operatorname{cf}^{V[G]}(\kappa)<\kappa$. Then, by Lemma $3.9(\mathrm{~b}), \kappa$ is collapsed in $V[G]$, which is impossible by (a).

(d) follows from (c).

Using the previous lemma and Theorem 3.5 we obtain

Theorem 3.11 Assume that $0^{\sharp}$ does not exist, and let $\mathbb{B}$ be a complete Boolean algebra and $2^{<\mathfrak{h}_{2}(\mathbb{B})}=\mathfrak{h}_{2}(\mathbb{B})$. Then White has a winning strategy in $\mathcal{G}_{\text {ls }}\left(\mathfrak{h}_{2}(\mathbb{B})\right)$ iff forcing by $\mathbb{B}$ collapses $2^{\mathfrak{h}_{2}(\mathbb{B})}$ to $\mathfrak{h}_{2}(\mathbb{B})$ in some generic extension.

Now, for a singular cardinal $\kappa$, we compare the games $\mathcal{G}_{\mathrm{ls}}(\kappa)$ and $\mathcal{G}_{\mathrm{ls}}(\operatorname{cf}(\kappa))$ from the aspect of White. 
Theorem 3.12 Let $\kappa \geq \omega$ be a cardinal, $\lambda=\operatorname{cf}(\kappa)<\kappa$ and $\mathbb{B}$ a complete Boolean algebra. If White has a winning strategy in the game $\mathcal{G}_{\mathrm{ls}}(\kappa)$ played on $\mathbb{B}$, then White has a winning strategy in $\mathcal{G}_{\mathrm{ls}}(\lambda)$ as well.

Proof. Suppose White has a winning strategy in $\mathcal{G}_{\text {ls }}(\kappa)$. Then, according to Theorem 3.4, there are $p \in B^{+}$and $w:{ }^{<\kappa} 2 \rightarrow[0, p]_{\mathbb{B}}$ satisfying (3.1). Let $\left\langle\alpha_{\xi}: \xi<\lambda\right\rangle$ be an increasing and cofinal sequence in $\kappa$ and let us define $\alpha_{\lambda}=\kappa$. We define a function $f: \lambda_{2} \rightarrow \leq \kappa 2$ as follows. For $\varphi: \zeta \rightarrow 2$, where $\zeta \leq \lambda$, let $f(\varphi): \alpha_{\zeta} \rightarrow 2$ be defined by:

$$
f(\varphi)(\alpha)= \begin{cases}\varphi(\xi) & \text { if } \alpha=\alpha_{\xi}, \text { for some } \xi<\zeta \\ 0 & \text { if } \alpha \in \alpha_{\zeta} \backslash\left\{\alpha_{\xi}: \xi<\zeta\right\}\end{cases}
$$

Let the function $v:<\lambda_{2} \rightarrow[0, p]_{\mathbb{B}}$ be defined by $v(\varphi)=w(f(\varphi))$. According to Theorem 3.4 it remains to be proved that

$$
\forall j: \lambda \rightarrow 2 \bigwedge_{\zeta<\lambda} \bigvee_{\xi \geq \zeta} v_{j \mid \xi}^{j(\xi)}=0
$$

Let $j: \lambda \rightarrow 2$. Then $i=f(j): \kappa \rightarrow 2$ and it is easy to check that $f(j \uparrow \xi)=$ $i\left\lceil\alpha_{\xi}\right.$, for all $\xi<\lambda$. Hence for each $\xi<\lambda$ we have $v_{j\lceil\xi}^{j(\xi)}=w_{f(j\lceil\xi)}^{j(\xi)}=w_{i\left\lceil\alpha_{\xi}\right.}^{i\left(\alpha_{\xi}\right)}$. Consequently, $\bigvee_{\xi \geq \zeta} v_{j \uparrow \xi}^{j(\xi)}=\bigvee_{\xi \geq \zeta} w_{i \uparrow \alpha_{\xi}}^{i\left(\alpha_{\xi}\right)} \leq \bigvee_{\alpha \geq \alpha_{\zeta}} w_{i \uparrow \alpha}^{i(\alpha)}$ holds for each $\zeta<\lambda$. So, according to (3.1),

$$
\bigwedge_{\zeta<\lambda} \bigvee_{\xi \geq \zeta} v_{j \zeta \xi}^{j(\xi)} \leq \bigwedge_{\zeta<\lambda} \bigvee_{\alpha \geq \alpha_{\zeta}} w_{i \uparrow \alpha}^{i(\alpha)}=\bigwedge_{\beta<\kappa} \bigvee_{\alpha \geq \beta} w_{i \uparrow \alpha}^{i(\alpha)}=0
$$

and (3.14) is proved.

However, we don't know is it possible at all for White to have a winning strategy on a singular cardinal.

Lemma 3.13 For every complete Boolean algebra $\mathbb{B}$ we have $(\mathrm{a}) \Rightarrow(\mathrm{b}) \Rightarrow(\mathrm{c})$, where

(a) White has a winning strategy in the game $\mathcal{G}_{\mathrm{ls}}(\omega)$;

(b) White has a winning strategy in the game $\mathcal{G}_{\text {dist }}\left(\omega, \omega_{1}, \omega_{1}\right)$;

(c) White has a winning strategy in the game $\mathcal{G}_{\text {dist }}\left(\omega, \omega_{1}, \omega\right)$.

Proof. The implication $(b) \Rightarrow(c)$ is evident. Suppose that (a) holds. Then,

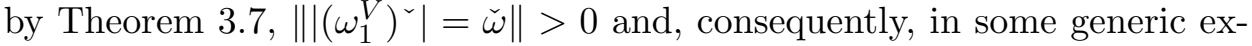
tension $V_{\mathbb{B}}[G]$, there is a bijection $x: \omega \rightarrow \omega_{1}^{V}$. In $V$, for each $s: \omega \rightarrow\left[\omega_{1}\right]^{<\omega_{1}}$ the set $\bigcup_{n \in \omega} s(n)$ is a bounded subset of $\omega_{1}$ so there is $n \in \omega$ such that 
$x(n) \notin s(n)$. Thus the algebra $\mathbb{B}$ is not $\left(\omega, \omega_{1}, \omega_{1}\right)$-distributive and, by Theorem 2.39 , we have (b).

We remark that in $\mathcal{G}_{\text {dist }}\left(\omega, \omega_{1}, \omega_{1}\right)$ Black has a winning strategy on every c.c.c. Boolean algebra, since he can choose all elements White offers him. The game $\mathcal{G}_{\text {dist }}\left(\omega, \omega_{1}, \omega\right)$ can not be equivalent to either of the games $\mathcal{G}_{\text {ls }}(\omega)$ and $\mathcal{G}_{\text {dist }}\left(\omega, \omega_{1}, \omega_{1}\right)$, from the aspect of either of the players. This is because on the Cohen algebra $\mathbb{B}$ White wins $\mathcal{G}_{\text {dist }}\left(\omega, \omega_{1}, \omega\right)$ (since forcing with $\mathbb{B}$ adds an unbounded real, and therefore $\mathbb{B}$ is not even $(\omega, \omega, \omega)$-distributive), and Black wins $\mathcal{G}_{\mathrm{ls}}(\omega)$ (by Corollary 3.15) and $\mathcal{G}_{\text {dist }}\left(\omega, \omega_{1}, \omega_{1}\right)$ (because $\mathbb{B}$ is c.c.c.). Also, the games $\mathcal{G}_{\mathrm{ls}}(\omega)$ and $\mathcal{G}_{\text {dist }}\left(\omega, \omega_{1}, \omega_{1}\right)$ are not equivalent from the aspect of Black, since on the Suslin algebra constructed in Theorem 3.23 Black does not have a winning strategy in $\mathcal{G}_{\mathrm{ls}}(\omega)$, and he has one in $\mathcal{G}_{\text {dist }}\left(\omega, \omega_{1}, \omega_{1}\right)$ (again because the algebra is c.c.c.).

\subsection{The game $\mathcal{G}_{\mathrm{ls}}(\kappa)$ from Black's point of view}

Theorem 3.14 If $\mathbb{B}$ is a complete Boolean algebra and $\kappa \geq \pi(\mathbb{B})$, then Black has a winning strategy in the game $\mathcal{G}_{\mathrm{ls}}(\kappa)$ played on $\mathbb{B}$.

Proof. Let $\lambda=\pi(\mathbb{B})$ and let $D \in\left[B^{+}\right]^{\lambda}$ be dense in $\mathbb{B}$. We define a strategy $\Sigma$ for Black. At the beginning White chooses $p \in B^{+}$and Black takes a (not necessarily one-to-one) enumeration of the members of $D$ which are below $p,\{d \in D: d \leq p\}=\left\{d_{\delta}: \delta \in \lambda\right\}$ and makes a sequence $\left\langle e_{\alpha}: \alpha<\kappa\right\rangle$ of elements of the set $\left\{d_{\delta}: \delta \in \lambda\right\}$ in which each $d_{\delta}$ appears $\kappa$-many times. (For example, Black can take a bijection $f: \kappa \rightarrow \lambda \times \kappa$ and define $e_{\alpha}=d_{\pi_{1}(f(\alpha))}$, for $\alpha \in \kappa$. Then $e_{\alpha}=d_{\delta}$ for all $\alpha \in f^{-1}[\{\delta\} \times \kappa] \in[\kappa]^{\kappa}$.) In the $\alpha$-th move, when White plays $p_{\alpha} \in(0, p)_{\mathbb{B}}$, Black responds choosing $i_{\alpha} \in 2$ such that $e_{\alpha} \wedge p_{\alpha}^{i_{\alpha}}>0$.

We prove that $\Sigma$ is a winning strategy for Black. Let us consider a play $\left\langle p, p_{0}, i_{0}, \ldots, p_{\alpha}, i_{\alpha}, \ldots\right\rangle$ in which Black follows $\Sigma$. For $\beta \in \kappa$ let $q_{\beta}=$ $\bigvee_{\alpha \geq \beta} p_{\alpha}^{i_{\alpha}}$. Let us prove

$$
\forall \delta \in \lambda \forall \beta \in \kappa d_{\delta} \wedge q_{\beta}>0 .
$$

Let $\delta \in \lambda$ and $\beta \in \kappa$. Since $d_{\delta}$ appears in the sequence $\left\langle e_{\alpha}\right\rangle \kappa$-many times, there is $\alpha \geq \beta$ such that $e_{\alpha}=d_{\delta}$, thus $d_{\delta} \wedge p_{\alpha}^{i_{\alpha}}>0$, which implies $d_{\delta} \wedge q_{\beta}>0$ and (3.15) is proved.

Let $\beta \in \kappa$. For each $q \in(0, p]_{\mathbb{B}}$ there is $\delta \in \lambda$ such that $d_{\delta} \leq q$ and since by (3.15) $d_{\delta} \wedge q_{\beta}>0$, we have $q \wedge q_{\beta}>0$. Thus $q \wedge q_{\beta}>0$ for all $q \in(0, p]_{\mathbb{B}}$, 
which implies $q_{\beta}=p$. So $q_{\beta}=p$ for all $\beta \in \kappa$, hence $\bigwedge_{\beta \in \kappa} \bigvee_{\alpha \geq \beta} p_{\alpha}^{i_{\alpha}}=p>0$ and Black wins the game.

Taking into account Lemma 1.76, for $\kappa=\omega$ the previous theorem takes the following form:

Corollary 3.15 Black has a winning strategy in the game $\mathcal{G}_{\mathrm{ls}}(\omega)$ played on the Cohen algebra.

Theorem 3.16 If a complete Boolean algebra $\mathbb{B}$ contains a $\lambda$-closed dense subset $D \subseteq B^{+}$, then for each infinite cardinal $\kappa<\lambda$ Black has a winning strategy in the game $\mathcal{G}_{\mathrm{ls}}(\kappa)$.

Proof. Follows from Theorem 2.34 and Lemma 3.3.

Theorem 2.23 applied to the game $\mathcal{G}_{\mathrm{ls}}(\kappa)$ gives us

Theorem 3.17 Let $\kappa \geq \omega$ be a cardinal and $\mathbb{B}$ a complete Boolean algebra. Then the following conditions are equivalent:

(a) Black has a winning strategy in the game $\mathcal{G}_{\mathrm{ls}}(\kappa)$ played on $\mathbb{B}$;

(b) for each $p \in B^{+}$there is $i: \bigcup_{\alpha<\kappa}{ }^{\alpha+1}(0, p)_{\mathbb{B}} \rightarrow 2$ such that for each sequence $\left\langle p_{\alpha}: \alpha<\kappa\right\rangle$ in $(0, p)_{\mathbb{B}}$

$$
\bigwedge_{\beta<\kappa} \bigvee_{\alpha \geq \beta} p_{\alpha}^{i\left(\left\langle p_{\delta}: \delta \leq \alpha\right\rangle\right)}>0
$$

(c) for each $p \in B^{+}$there is $i: \bigcup_{\alpha<\kappa}{ }^{\alpha+1}[0, p]_{\mathbb{B}} \rightarrow 2$ such that for each sequence $\left\langle p_{\alpha}: \alpha<\kappa\right\rangle$ in $[0, p]_{\mathbb{B}}(3.16)$ holds.

As in Theorem 3.12 we compare the games $\mathcal{G}_{\mathrm{ls}}(\kappa)$ and $\mathcal{G}_{\mathrm{ls}}(\operatorname{cf}(\kappa))$, where $\kappa$ is a singular cardinal, but now from Black's point of view.

Theorem 3.18 Let $\kappa \geq \omega$ be a cardinal, $\lambda=\operatorname{cf}(\kappa)<\kappa$ and $\mathbb{B}$ a complete Boolean algebra. If Black has a winning strategy in the game $\mathcal{G}_{\mathrm{ls}}(\lambda)$ played on $\mathbb{B}$, then Black has a winning strategy in the game $\mathcal{G}_{\mathrm{ls}}(\kappa)$ as well.

Proof. According to Theorem 3.17, for arbitrary $p \in B^{+}$it suffices to construct a function $i: \bigcup_{\alpha<\kappa}{ }^{\alpha+1}(0, p)_{\mathbb{B}} \rightarrow 2$ such that for each sequence $\left\langle p_{\alpha}: \alpha<\kappa\right\rangle$ in $(0, p)_{\mathbb{B}}(3.16)$ holds. By the assumption and according to 
Theorem 3.17 there is $j: \bigcup_{\xi<\lambda}{ }^{\xi+1}(0, p)_{\mathbb{B}} \rightarrow 2$ such that for each sequence $\left\langle q_{\xi}: \xi<\lambda\right\rangle$ in $(0, p)_{\mathbb{B}}$

$$
\bigwedge_{\zeta<\lambda} \bigvee_{\xi \geq \zeta} q_{\xi}^{j\left(\left\langle q_{\gamma}: \gamma \leq \xi\right\rangle\right)}>0
$$

Let $\left\langle\alpha_{\xi}: \xi<\lambda\right\rangle$ be an increasing sequence cofinal in $\kappa$. Using the function $j$ we define $i$ by

$$
i\left(\left\langle p_{\delta}: \delta \leq \alpha\right\rangle\right)= \begin{cases}0 & \text { if } \alpha \notin\left\{\alpha_{\xi}: \xi<\lambda\right\} \\ j\left(\left\langle p_{\alpha_{\gamma}}: \gamma \leq \xi\right\rangle\right) & \text { if } \alpha=\alpha_{\xi} \text { for some } \xi<\lambda\end{cases}
$$

Now we prove $(3.16)$ for an arbitrary sequence $\left\langle p_{\alpha}: \alpha<\kappa\right\rangle$ in $(0, p)_{\mathbb{B}}$. Since the sequence $\left\langle\alpha_{\xi}: \xi<\lambda\right\rangle$ is unbounded in $\kappa$ we have (introducing $q_{\xi}=p_{\alpha_{\xi}}$ for $\xi<\lambda)$

$$
\begin{aligned}
\bigwedge_{\beta<\kappa} \bigvee_{\alpha \geq \beta} p_{\alpha}^{i\left(\left\langle p_{\delta}: \delta \leq \alpha\right\rangle\right)} & =\bigwedge_{\zeta<\lambda} \bigvee_{\alpha \geq \alpha_{\zeta}} p_{\alpha}^{i\left(\left\langle p_{\delta}: \delta \leq \alpha\right\rangle\right)} \\
& \geq \bigwedge_{\zeta<\lambda} \bigvee_{\xi \geq \zeta} p_{\alpha_{\xi}}^{i\left(\left\langle p_{\delta}: \delta \leq \alpha_{\xi}\right\rangle\right)} \\
& =\bigwedge_{\zeta<\lambda} \bigvee_{\xi \geq \zeta} p_{\alpha_{\xi}}^{j\left(\left\langlep_{\left.\left.\alpha_{\gamma}: \gamma \leq \xi\right\rangle\right)}\right.\right.} \\
& =\bigwedge_{\zeta<\lambda} \bigvee_{\xi \geq \zeta} q_{\xi}^{j\left(\left\langle q_{\gamma}: \gamma \leq \xi\right\rangle\right)}
\end{aligned}
$$

Now (3.17) implies (3.16).

The following theorem considers only the game $\mathcal{G}_{\mathrm{ls}}(\omega)$, and it is related to Maharam $^{1}$ algebras.

Definition 3.19 A submeasure on a complete Boolean algebra $\mathbb{B}$ is a function $\nu: B \rightarrow[0, \infty)$ satisfying:

(i) $\nu(0)=0$;

(ii) $a \leq b \Rightarrow \nu(a) \leq \nu(b)$

(iii) $\nu(a \vee b) \leq \nu(a)+\nu(b)$.

A submeasure $\nu$ is strictly positive if

(iv) $a>0 \Rightarrow \nu(a)>0$.

A submeasure $\nu$ is a Maharam (also called continuous) submeasure if

(v) for each sequence $\left\langle a_{n}: n \in \omega\right\rangle$ in $B$, if $a_{0} \geq a_{1} \geq \ldots$ and $\bigwedge_{n \in \omega} a_{n}=0$ then $\lim _{n \rightarrow \infty} \nu\left(a_{n}\right)=0$.

A complete Boolean algebra is called a Maharam algebra if it carries a strictly positive Maharam submeasure.

\footnotetext{
${ }^{1}$ Dorothy Maharam Stone (b. 1917), American mathematician
} 
Theorem 3.20 If $\mathbb{B}$ is a Maharam algebra, then Black has a winning strategy in the game $\mathcal{G}_{\mathrm{ls}}(\omega)$ played on $\mathbb{B}$.

Proof. Let $\nu$ be a strictly positive Maharam submeasure on $\mathbb{B}$. We define a strategy $\Sigma$ for Black. At the beginning White plays $p \in B^{+}$and, by (iv), $\nu(p)>0$. If in the $n$-th move White plays $p_{n} \in(0, p)_{\mathbb{B}}$ then by (iii), $\nu(p) \leq$ $\nu\left(p_{n}\right)+\nu\left(p \backslash p_{n}\right)$ and consequently $\nu\left(p_{n}\right) \geq \nu(p) / 2$ or $\nu\left(p \backslash p_{n}\right) \geq \nu(p) / 2$. Then Black plays $i_{n} \in 2$ such that $\nu\left(p_{n}^{i_{n}}\right) \geq \nu(p) / 2$.

Let us show that $\Sigma$ is a winning strategy for Black. Let $\left\langle p, p_{0}, i_{0}, \ldots\right\rangle$ be an arbitrary play in which Black follows $\Sigma$ and for $k \in \omega$ let $q_{k}=\bigvee_{n \geq k} p_{n}^{i_{n}}$. Then, according to (ii) we have $\nu\left(q_{k}\right) \geq \nu\left(p_{k}^{i_{k}}\right) \geq \nu(p) / 2$. Suppose $\bigwedge_{k \in \omega} q_{k}=$ 0 . Then, since $q_{0} \geq q_{1} \geq \ldots$ and (v) holds, we obtain $\lim _{n \rightarrow \infty} \nu\left(q_{k}\right)=0$, which is impossible because $\nu\left(q_{k}\right) \geq \nu(p) / 2>0$. So $\bigwedge_{k \in \omega} \bigvee_{n \geq k} p_{n}^{i_{n}}>0$ and Black wins.

It is well-known that every Maharam algebra is c.c.c. and weakly distributive. So, concerning Theorem 3.20, it is natural to ask whether Black has a winning strategy on each weakly distributive c.c.c. complete Boolean algebra. The answer "Yes" is consistent. Namely, Balcar², Jech and Pazák ${ }^{3}$ in [2] and, independently, Veličković in [37] proved that the P-ideal dichotomy (a principle formulated by Abraham $^{4}$ and Todorčević ${ }^{5}$ in [1] and extended by Todorčević in [35]) implies each weakly distributive c.c.c. complete Boolean algebra is a Maharam algebra. Similar results are also obtained by Farah ${ }^{6}$ and Zapletal in [11]. But the answer "No" is consistent as well since in the following section we construct a c.c.c. $\omega$-distributive algebra without a winning strategy for Black in the game $\mathcal{G}_{\mathrm{ls}}(\omega)$.

\subsection{Some additional results}

For a complete Boolean algebra $\mathbb{B}$, we define

White $(\mathbb{B})=\left\{\kappa \in\right.$ Card $\backslash \omega:$ White has a winning strategy in $\mathcal{G}_{\mathrm{ls}}(\kappa)$ on $\left.\mathbb{B}\right\}$

$\operatorname{Black}(\mathbb{B})=\left\{\kappa \in \operatorname{Card} \backslash \omega:\right.$ Black has a winning strategy in $\mathcal{G}_{\mathrm{ls}}(\kappa)$ on $\left.\mathbb{B}\right\}$.

\footnotetext{
${ }^{2}$ Bohuslav Balcar (b. 1943), Czech mathematician

${ }^{3}$ Tomáš Pazák, contemporary Czech mathematician

${ }^{4}$ Uri Abraham, contemporary Israeli mathematician

${ }^{5}$ Stevo Todorčević (b. 1955), Serbian mathematician

${ }^{6}$ Ilias Farah, contemporary Serbian mathematician
} 
In contrast to Lemma $2.30\left(\right.$ a) for $\mathcal{G}_{\text {dist }}(\kappa, 2,2)$, Theorems 3.16 and 3.14 imply that Black has a winning strategy in $\mathcal{G}_{\mathrm{ls}}(\kappa)$ for both small and large values of $\kappa$, and the cardinals $\kappa$ for which White has a winning strategy in $\mathcal{G}_{\text {ls }}(\kappa)$ belong to a bounded interval determined by some cardinal invariants of $\mathbb{B}$ :

Theorem 3.21 White $(\mathbb{B}) \subseteq\left[\mathfrak{h}_{2}(\mathbb{B}), \pi(\mathbb{B})\right)$ holds for every complete Boolean algebra $\mathbb{B}$.

Proof. The inequality $\kappa \geq \mathfrak{h}_{2}(\mathbb{B})$ follows from Corollary 2.33 and Lemma $3.3(\mathrm{a})$ and $\kappa<\pi(\mathbb{B})$ from Theorem 3.14.

Example 3.1 (The converse of neither Theorem 3.12 nor Theorem 3.18 is true.) Let $V \models \mathrm{CH}$ and, in $V$, let $\mathbb{B}=\operatorname{Col}\left(\omega, \omega_{1}\right)$ (Example 1.2). Since $1 \Vdash_{\mathbb{B}}\left|\left(\left(2^{\omega}\right)^{V}\right)^{\smile}\right|=\check{\omega}$, according to Corollary 3.7, White has (thus, Black has not) a winning strategy in the game $\mathcal{G}_{\mathrm{ls}}(\omega)$ played on $\mathbb{B}$. But, since $\aleph_{\omega} \geq \pi(\mathbb{B})=\omega_{1}$, according to Theorem 3.14 , Black has a winning strategy in $\mathcal{G}_{\mathrm{ls}}\left(\aleph_{\omega}\right)$.

Example 3.2 (Black can have a winning strategy on a collapsed cardinal.) Let $V \models \mathrm{MA}+2^{\omega}=\aleph_{\omega+1}$ (see Theorem 1.81). Then, by Lemma 1.25, in $V$ we have $2^{\kappa}=\aleph_{\omega+1}$ for each infinite cardinal $\kappa \leq \aleph_{\omega}$. In $V$, let $\mathbb{B}=\operatorname{Col}\left(\omega_{1}, \aleph_{\omega+1}\right)$. Then $1 \Vdash_{\mathbb{B}}\left|\left(\left(2^{\omega_{1}}\right)^{V}\right)^{`}\right|=\left(\omega_{1}^{V}\right)^{`} \in$ Reg so by Theorem 3.5 White has a winning strategy in the game $\mathcal{G}_{\mathrm{ls}}\left(\omega_{1}\right)$. On the other hand, $\mathbb{B}$ has an $\omega_{1}$-closed dense subset $<\omega_{1} \omega_{\omega+1}$, so, according to Theorem 3.16 , Black has a winning strategy in the game $\mathcal{G}_{\mathrm{ls}}(\omega)$ and, according to Theorem 3.18 , in the game $\mathcal{G}_{\mathrm{ls}}\left(\aleph_{\omega}\right)$, although the cardinal $\aleph_{\omega}$ is collapsed to $\aleph_{1}$ in each generic extension by $\mathbb{B}$.

Example 3.3 (The implication $(\mathrm{c}) \Rightarrow(\mathrm{b})$ in Theorem 3.5 does not hold.) Let $V$ and $\mathbb{B}$ be as in Example 3.2. Then in $V$, for $\kappa=\aleph_{\omega}$ we have $2^{\aleph_{\omega}}=$ $2^{<\aleph_{\omega}}=\mathfrak{c}=\aleph_{\omega+1}$, so the sets $\left({ }^{\kappa} 2\right)^{V}$ and $\left({ }^{<\kappa} 2\right)^{V}$ are equipotent in $V_{\mathbb{B}}[G]$ as well. But White does not have a winning strategy in the game $\mathcal{G}_{\mathrm{ls}}\left(\aleph_{\omega}\right)$, since Black has one.

Example 3.4 (The games $\mathcal{G}_{\mathrm{ls}}(\omega)$ and $\mathcal{G}_{\text {dist }}(\omega, \mathfrak{c}, \omega)$ are never equivalent for Black.) Let $\mathbb{B}$ be the Cohen algebra. By Corollary 3.15 Black has a winning strategy in the game $\mathcal{G}_{\mathrm{ls}}(\omega)$ played on $\mathbb{B}$. Suppose Black has a winning strategy $\Sigma$ in $\mathcal{G}_{\text {dist }}(\omega, \mathfrak{c}, \omega)$ on $\mathbb{B}$. Then $\Sigma$ is also a winning strategy for Black in the game $\mathcal{G}_{\text {dist }}(\omega, \omega, \omega)$ so White does not have a winning strategy in $\mathcal{G}_{\text {dist }}(\omega, \omega, \omega)$ According to Theorem 2.39 this implies that $\mathbb{B}$ is weakly 
distributive. But this contradicts Corollary 1.66 since the Cohen algebra adds an unbounded real. Thus Black does not have a winning strategy in the game $\mathcal{G}_{\text {dist }}(\omega, \mathfrak{c}, \omega)$.

In the following theorem we show that the set White $(\mathbb{B})$ need not be convex. Moreover, under some additional assumptions, regarding the set White $(\mathbb{B}) \cap$ Reg "everything is possible".

Theorem 3.22 (GCH) For each set $S$ of regular cardinals there is a complete Boolean algebra $\mathbb{B}$ such that

(a) White $(\mathbb{B})=S$;

(b) $\operatorname{Black}(\mathbb{B})=\operatorname{Card} \backslash(S \cup \omega)$.

Proof. If $S=\emptyset$, let $\mathbb{B}=$ r.o. $\left(\left\langle<\omega_{2}, \supseteq\right\rangle\right)$ (the Cohen algebra). Since $\mathfrak{h}_{2}(\mathbb{B})=$ $\pi(\mathbb{B})=\omega$, according to Theorem 3.21 we have White $(\mathbb{B})=\emptyset$. On the other hand, by Theorem 3.14, Black $(\mathbb{B})=\operatorname{Card} \backslash \omega$.

If $S \neq \emptyset$, for each $\kappa \in S$ let $\mathbb{B}_{\kappa}=\operatorname{Col}\left(\kappa, \kappa^{+}\right)=$r.o. $\left(\left\langle<\kappa \kappa^{+}, \supseteq\right\rangle\right)$. The regularity of $\kappa$ implies that $<\kappa \kappa^{+}$is a $\kappa$-closed dense subset of $\mathbb{B}_{\kappa}$, and thus, according to Corollary 1.58,

$$
1_{\mathbb{B}_{\kappa}} \Vdash_{\mathbb{B}_{\kappa}} \check{\kappa} \in \operatorname{Reg}
$$

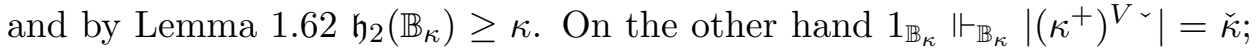
by Lemma $3.8 \kappa$ obtains new subsets in each generic extension by $\mathbb{B}_{\kappa}$, which by Theorem 1.59 implies

$$
\mathfrak{h}_{2}(\mathbb{B})=\kappa \text {. }
$$

GCH implies $\left.\right|^{<\kappa} \kappa^{+} \mid=\kappa^{+}$, hence $\pi\left(\mathbb{B}_{\kappa}\right) \leq \kappa^{+}$. But $\mathbb{B}_{\kappa}$ is not $\kappa^{+}$-c.c. so

$$
\pi(\mathbb{B})=\kappa^{+} .
$$

According to (3.19), (3.20) and Theorem 3.21 we have White $\left(\mathbb{B}_{\kappa}\right) \subseteq\{\kappa\}$. Since $1_{\mathbb{B}_{\kappa}} \Vdash_{\mathbb{B}_{\kappa}}\left|\left(\left(2^{\kappa}\right)^{V}\right)^{\smile}\right|=\check{\kappa}$ and (3.18) holds, Theorem 3.6 implies $\kappa \in$ White $\left(\mathbb{B}_{\kappa}\right)$ so

$$
\text { White }\left(\mathbb{B}_{\kappa}\right)=\{\kappa\} \text {. }
$$

We prove that the algebra $\mathbb{B}=\prod_{\kappa \in S} \mathbb{B}_{\kappa}$ (the usual direct product of algebras $\left.\mathbb{B}_{\kappa}\right)$ satisfies the required properties. It suffices to prove that $(1)$ White $(\mathbb{B}) \supseteq$ $S ;(2) \operatorname{Black}(\mathbb{B}) \supseteq \operatorname{Card} \backslash(S \cup \omega)$.

(1) Let $\kappa \in S$. By $(3.21) \kappa \in$ White $\left(\mathbb{B}_{\kappa}\right)$ and, according to (3.18) and Theorem 3.6 there is an extension $V_{\mathbb{B}_{\kappa}}[H]$ such that

$$
\left|\left(2^{\kappa}\right)^{V}\right|^{V_{\mathbb{B}_{\kappa}}[H]}=\kappa \in \operatorname{Reg}^{V_{\mathbb{B}_{\kappa}}[H]} .
$$


By Lemma 1.50(b) there is a $\mathbb{B}$-generic filter $G$ over $V$ such that $V_{\mathbb{B}_{\kappa}}[H]=$ $V_{\mathbb{B}}[G]$ so, by $(3.22),\left|\left(2^{\kappa}\right)^{V}\right|^{V_{\mathbb{B}}[G]}=\kappa \in \operatorname{Reg}^{V_{\mathbb{B}}[G]}$ so, according to Theorem $3.5, \kappa \in$ White $(\mathbb{B})$.

(2) Let $\lambda \in$ Card $\backslash(S \cup \omega)$. We define a strategy $\Sigma_{1}$ for Black in the game $\mathcal{G}_{\mathrm{ls}}(\lambda)$ played on $\mathbb{B}=\prod_{\kappa \in S} \mathbb{B}_{\kappa}$, as follows.

Let $p=\left\langle b_{\kappa}: \kappa \in S\right\rangle \in B^{+}$be an initial move of White. Then $b_{\kappa}>0_{\mathbb{B}_{\kappa}}$ for some $\kappa \in S$, so for $\kappa_{0}=\min \left\{\kappa \in S: b_{\kappa}>0_{\mathbb{B}_{\kappa}}\right\}$ we have $b_{\kappa_{0}}>0_{\mathbb{B}_{\kappa_{0}}}$ and, since $\lambda \notin S, \lambda \neq \kappa_{0}$. Now there are two cases.

$1^{\circ} \lambda<\kappa_{0}$. Since the algebra $\mathbb{B}_{\kappa_{0}}$ contains a dense $\kappa_{0}$-closed subset, according to Theorem 3.16 Black has a winning strategy in the game $\mathcal{G}_{\text {ls }}(\lambda)$ played on $\mathbb{B}_{\kappa_{0}}$.

$2^{\circ} \lambda>\kappa_{0}$. Then, according to $(3.20), \lambda \geq \pi\left(\mathbb{B}_{\kappa_{0}}\right)$ so, by Theorem 3.14, Black has a winning strategy $\Sigma$ in the game $\mathcal{G}_{\text {ls }}(\lambda)$ on $\mathbb{B}_{\kappa_{0}}$.

Let $i^{\prime}: \bigcup_{\alpha<\kappa}{ }^{\alpha+1}[0, p]_{\mathbb{B}} \rightarrow 2$ be the function provided by Theorem 3.17(c). We define

$$
i\left(\left\langle p_{\beta}: \beta \leq \alpha\right\rangle\right)=i^{\prime}\left(\left\langle\pi_{\kappa_{0}}\left(p_{\beta}\right): \beta \leq \alpha\right\rangle\right),
$$

where $\pi_{\kappa_{0}}: \prod_{\kappa \in S} \mathbb{B}_{\kappa} \rightarrow \mathbb{B}_{\kappa_{0}}$ is the projection of $\mathbb{B}$ onto its factor $\mathbb{B}_{\kappa_{0}}$.

We prove that $i$ satisfies (3.16) as well. Let $\left\langle p_{\alpha}: \alpha<\lambda\right\rangle \in{ }^{\lambda}[0, p]_{\mathbb{B}}$ and $i_{\alpha}=i\left(\left\langle p_{\beta}: \beta \leq \alpha\right\rangle\right)$ for $\alpha<\lambda$. By the choice of $i^{\prime}$ we have

$$
\bigwedge_{\beta<\kappa} \bigvee_{\alpha \geq \beta} \pi_{\kappa_{0}}\left(p_{\alpha}\right)^{i_{\alpha}}>0
$$

(we remind the reader that $\pi_{\kappa_{0}}\left(p_{\alpha}\right)^{0}=\pi_{\kappa_{0}}\left(p_{\alpha}\right)$ and $\pi_{\kappa_{0}}\left(p_{\alpha}\right)^{1}=\pi_{\kappa_{0}}(p) \backslash$ $\left.\pi_{\kappa_{0}}\left(p_{\alpha}\right)\right)$. Now since $\pi_{\kappa_{0}}$ is a homomorphism, we have $\pi_{\kappa_{0}}\left(p_{\alpha}^{0}\right)=\pi_{\kappa_{0}}\left(p_{\alpha}\right)=$ $\pi_{\kappa_{0}}\left(p_{\alpha}\right)^{0}$ and $\pi_{\kappa_{0}}\left(p_{\alpha}^{1}\right)=\pi_{\kappa_{0}}\left(p \wedge p_{\alpha}^{\prime}\right)=\pi_{\kappa_{0}}(p) \backslash \pi_{\kappa_{0}}\left(p_{\alpha}\right)=\pi_{\kappa_{0}}\left(p_{\alpha}\right)^{1}$, thus we have $\pi_{\kappa_{0}}\left(p_{\alpha}^{i_{\alpha}}\right)=\pi_{\kappa_{0}}\left(p_{\alpha}\right)^{i_{\alpha}}$. Now, according to (3.23) and the fact that $\pi_{\kappa_{0}}$ is a complete homomorphism, we have

$$
\pi_{\kappa_{0}}\left(\bigwedge_{\beta<\lambda} \bigvee_{\alpha \geq \beta} p_{\alpha}^{i_{\alpha}}\right)=\bigwedge_{\beta<\lambda \alpha \geq \beta} \bigvee_{\beta} \pi_{\kappa_{0}}\left(p_{\alpha}\right)^{i_{\alpha}}>0
$$

which implies that $\bigwedge_{\beta<\lambda} \bigvee_{\alpha \geq \beta} p_{\alpha}^{i_{\alpha}}>0$ in $\mathbb{B}$, so Black wins the game. Thus $\Sigma_{1}$ is indeed a winning strategy for Black.

\subsection{The indeterminacy}

We already mentioned that Jech, under assumption of $\diamond$, found a Boolean algebra on which the game $\mathcal{G}_{\text {dist }}(\omega, 2,2)$ is undetermined. Dobrinen in [9] and [10] generalized his result to the game $\mathcal{G}_{\text {dist }}(\kappa, 2,2)$ assuming $\kappa^{<\kappa}=\kappa$ 
and $\nabla_{\kappa^{+}}(E(\kappa))$. Modifying her proof, we will obtain a similar result for $\mathcal{G}_{\mathrm{ls}}(\kappa)$. It was first proved in [25] for $\kappa=\omega$ and then generalized in [26].

Theorem 3.23 For each $\kappa \in$ Reg satisfying $\kappa^{<\kappa}=\kappa$ and $\nabla_{\kappa^{+}}(E(\kappa))$, there is a $\kappa^{+}$-Suslin tree $\mathbb{T}$ such that the game $\mathcal{G}_{\mathrm{ls}}(\kappa)$ is undetermined on the algebra $\mathbb{B}=$ r.o. $\left(\mathbb{T}^{*}\right)$.

By Lemma 1.80 the reversed tree of every well-pruned $\kappa^{+}$-Suslin tree $\mathbb{T}$ is $\kappa$-distributive, so Theorem 3.21 implies that White does not have a winning strategy in the game $\mathcal{G}_{\mathrm{ls}}(\kappa)$ played on $\mathbb{B}$. Thus, after constructing the tree our main task will be to show that Black does not have a winning strategy either. For both the construction and the proof we will need some auxiliary lemmas.

First we introduce the game $\mathcal{G}_{\mathrm{ls}}^{\prime \prime}(\kappa)$ in which White's initial move must be $p=1$ and all other rules are the same as in the game $\mathcal{G}_{\mathrm{ls}}(\kappa)$. This game is easier for Black:

Lemma 3.24 Let $\kappa \geq \omega$ be a cardinal and $\mathbb{B}$ a complete Boolean algebra. If Black has a winning strategy in the game $\mathcal{G}_{\text {ls }}(\kappa)$ played on $\mathbb{B}$, then he has a winning strategy in the game $\mathcal{G}_{\mathrm{ls}}^{\prime \prime}(\kappa)$ as well.

Proof. Applying Theorem 3.17 with $p=1$ we easily obtain the existence of a winning strategy for Black in $\mathcal{G}_{\mathrm{ls}}^{\prime \prime}(\kappa)$.

Thus it suffices to construct a well-pruned $\kappa^{+}$-Suslin tree $\mathbb{T}$ such that Black does not have a wining strategy in the game $\mathcal{G}_{\mathrm{ls}}^{\prime \prime}(\kappa)$ played on $\mathbb{B}=$ r.o. $\left(\mathbb{T}^{*}\right)$. Our construction will follow the standard construction of a $\kappa^{+}$Suslin tree, in which the set of nodes is $\kappa^{+}$. In order to provide a convenient coding of moves and strategies, we will construct our tree $\mathbb{T}$ so that for each $\beta<\kappa^{+}$the $\beta$-th level of $\mathbb{T}, \operatorname{Lev}_{\beta}(\mathbb{T})$, is the set $L_{\beta}=\{\kappa \beta+\gamma: \gamma<\kappa\}=$ $\kappa(\beta+1) \backslash \kappa \beta$.

We begin by coding all possible moves of White. Suppose that the intended well-pruned $\kappa^{+}$-Suslin tree $\mathbb{T}$ is constructed. Then each move $p$ of White in the game $\mathcal{G}_{\mathrm{ls}}^{\prime \prime}(\kappa)$ played on the algebra $\mathbb{B}=$ r.o. $\left(\mathbb{T}^{*}\right)$ is an element of the set $B^{+}$and, according to the following elementary fact, is determined by a subset of some level of $\mathbb{T}$.

Lemma 3.25 Let $\mathbb{T}=\langle T,<\rangle$ be a separative reversed well-pruned $\kappa^{+}$Suslin tree and $\mathbb{B}=$ r.o. $\left(\mathbb{T}^{*}\right)$. Then 
(a) if $t \in T$ and $\operatorname{ht}(t)<\beta<\kappa^{+}$, then (in $\left.\mathbb{B}\right) t=\bigvee\left\{s \in \operatorname{Lev}_{\beta}(\mathbb{T}): s<t\right\}$;

(b) for each $p \in B^{+}$there are $\beta<\kappa^{+}$and $W \subseteq \operatorname{Lev}_{\beta}(\mathbb{T})$ such that $p=\bigvee W$ and $p^{\prime}=\bigvee\left(\operatorname{Lev}_{\beta}(\mathbb{T}) \backslash W\right)$.

So the moves of White in the game $\mathcal{G}_{\mathrm{ls}}^{\prime \prime}(\kappa)$ can be regarded as elements of the set $\bigcup_{\beta<\kappa^{+}} P\left(\operatorname{Lev}_{\beta}(\mathbb{T})\right) \backslash\left\{\emptyset, \operatorname{Lev}_{\beta}(\mathbb{T})\right\}$ which is, by Lemma 1.7(b), of size $\kappa^{+}$. Since the levels $\operatorname{Lev}_{\beta}(\mathbb{T})$ of our tree will be the sets $L_{\beta}$, we can fix a bijection

$$
W: \kappa^{+} \rightarrow \bigcup_{\beta<\kappa^{+}} P\left(L_{\beta}\right) \backslash\left\{\emptyset, L_{\beta}\right\}
$$

which codes every move of White by some element of $\kappa^{+}$. (In fact each $p \in B^{+}$is coded by $\kappa^{+}$-many subsets of levels and, consequently, by $\kappa^{+}$many elements of $\kappa^{+}$, but this is irrelevant for the construction.)

Now we code the strategies of Black. According to Theorem 3.17 and using the bijection $W$ fixed above, to each strategy $\Sigma$ of Black for the game $\mathcal{G}_{\text {ls }}^{\prime \prime}(\kappa)$ on $\mathbb{B}=$ r.o. $\left(\mathbb{T}^{*}\right)$ we can adjoin a mapping $\bar{\Sigma}: \bigcup_{\alpha<\kappa}{ }^{\alpha+1} \kappa^{+} \rightarrow 2$, defined by

$$
\bar{\Sigma}\left(\left\langle\xi_{\delta}: \delta \leq \alpha\right\rangle\right)=\Sigma\left(\left\langle\bigvee W_{\xi_{\delta}}: \delta \leq \alpha\right\rangle\right) .
$$

Then clearly $\bar{\Sigma} \subseteq \bigcup_{\alpha<\kappa}{ }^{\alpha+1} \kappa^{+} \times 2$ and, under $\nabla_{\kappa^{+}}(E(\kappa)), \mid \bigcup_{\alpha<\kappa}{ }^{\alpha+1} \kappa^{+} \times$ $2 \mid=\Sigma_{\alpha<\kappa} 2^{\kappa|\alpha+1|}=\kappa^{+}$. Now we fix a bijection

$$
\Phi: \bigcup_{\alpha<\kappa}{ }^{\alpha+1} \kappa^{+} \times 2 \rightarrow \kappa^{+}
$$

and in this way each strategy $\Sigma$ of Black is coded by the subset $\Phi[\bar{\Sigma}]$ of $\kappa^{+}$.

Lemma 3.26 Let $\kappa$ be a regular cardinal satisfying $\kappa^{<\kappa}=\kappa$ and $\diamond_{\kappa^{+}}(E(\kappa))$ and let $C_{\Phi}=\left\{\delta<\kappa^{+}: \Phi\left[\bigcup_{\alpha<\kappa}{ }^{\alpha+1} \delta \times 2\right] \subseteq \delta\right\}$. Then there is a club subset $C^{*}$ of $\kappa^{+}$such that $C^{*} \cap E(\kappa) \subseteq C_{\Phi}$.

Proof. If $\xi<\kappa^{+}$we have $\left|\bigcup_{\alpha<\kappa}{ }^{\alpha+1}(\xi+1) \times 2\right| \leq \Sigma_{\alpha<\kappa} \kappa^{<\kappa}=\kappa$. Hence $\Phi\left[\bigcup_{\alpha<\kappa}{ }^{\alpha+1}(\xi+1) \times 2\right]$ is a bounded subset of $\kappa^{+}$so the function $f: \kappa^{+} \rightarrow \kappa^{+}$ given by $f(\xi)=\sup \Phi\left[\bigcup_{\alpha<\kappa}{ }^{\alpha+1}(\xi+1) \times 2\right]$ is well-defined and $C^{*}=\{\delta<$ $\left.\kappa^{+}: f[\delta] \subseteq \delta\right\}$ is a club subset of $\kappa^{+}$by Lemma 1.4 .

Let $\delta \in C^{*} \cap E(\kappa)$. If $\langle\varphi, k\rangle \in \bigcup_{\alpha<\kappa}{ }^{\alpha+1} \delta \times 2$, then there is $\alpha<\kappa$ such that $\varphi: \alpha+1 \rightarrow \delta$. Since $\operatorname{cf}(\delta)=\kappa>\alpha+1$ we have $\xi=\sup \varphi[\alpha+1]<\delta$ and $\delta \in C^{*}$ implies $f(\xi)<\delta$. Clearly $\varphi: \alpha+1 \rightarrow \xi+1$ so $\langle\varphi, k\rangle \in$ $\bigcup_{\alpha<\kappa}{ }^{\alpha+1}(\xi+1) \times 2$ and, by definition of $f, \Phi(\langle\varphi, k\rangle) \leq f(\xi) \in \delta$. Thus $\Phi\left[\bigcup_{\alpha<\kappa}{ }^{\alpha+1} \delta \times 2\right] \subseteq \delta$, that is $\delta \in C_{\Phi}$. 
Now we turn to describing the possible counter-moves of White. We will construct a $\kappa^{+}$-Suslin tree $\mathbb{T}=\left\langle\kappa^{+}, \triangleleft\right\rangle$ such that for each $\beta<\kappa^{+}$ $\operatorname{Lev}_{\beta}(\mathbb{T})=L_{\beta}$. For $\beta<\kappa^{+}$let $P_{\beta}$ be the set of even elements of the set $L_{\beta}$, that is $P_{\beta}=\left\{\alpha \in L_{\beta}: \exists \eta \in \operatorname{Lim} \exists n \in \omega \alpha=\eta+2 n\right\}$. Then clearly $P_{\beta}$ determines a move $\bigvee P_{\beta}$ of White in the game $\mathcal{G}_{\mathrm{ls}}^{\prime \prime}(\kappa)$ played on the algebra $\mathbb{B}=$ r.o. $\left(\mathbb{T}^{*}\right)$ and the sequence $\left\langle P_{\beta}: \beta<\kappa^{+}\right\rangle$can be regarded as a function

$$
P: \kappa^{+} \rightarrow \bigcup_{\beta<\kappa^{+}} P\left(L_{\beta}\right) \backslash\left\{\emptyset, L_{\beta}\right\} \text {. }
$$

If $W$ is the bijection fixed above and $g: \kappa^{+} \rightarrow \kappa^{+}$is defined by $g=W^{-1} \circ P$, then $\left\{\alpha<\kappa^{+}: g[\alpha] \subseteq \alpha\right\}$ is a club subset of $\kappa^{+}$by Lemma 1.4. Since $g[\alpha] \subseteq \alpha$ if and only if $P[\alpha] \subseteq W[\alpha]$, that is $\left\{P_{\beta}: \beta<\alpha\right\} \subseteq\left\{W_{\beta}: \beta<\alpha\right\}$, we have

Lemma 3.27 $C_{P}=\left\{\alpha<\kappa^{+}: P[\alpha] \subseteq W[\alpha]\right\}$ is a club subset of $\kappa^{+}$.

According to the definition of the function $g, P_{\beta}=W_{g(\beta)}$ for each $\beta<$ $\kappa^{+}$. So if in the first $\alpha+1$ moves of the game $\mathcal{G}_{\mathrm{ls}}^{\prime \prime}(\kappa)$ White plays the sequence $\left\langle\bigvee P_{\beta_{\delta}}: \delta \leq \alpha\right\rangle$, then Black, following a strategy $\Sigma$, responds by

$$
\bigvee P_{\beta_{\alpha}}^{\bar{\Sigma}\left(\left\langle g\left(\beta_{\delta}\right): \delta \leq \alpha\right\rangle\right)}
$$

where $\bar{\Sigma}$ is defined by (3.24) and for $W \subseteq \operatorname{Lev}_{\beta}(\mathbb{T})$, by definition, $W^{0}=W$ and $W^{1}=\operatorname{Lev}_{\beta}(\mathbb{T}) \backslash W$.

In our construction we will frequently use the following facts.

Lemma 3.28 If $\kappa$ is an uncountable cardinal, then $\kappa=\{\omega \zeta+n: \zeta<$ $\kappa \wedge n \in \omega\}$. Consequently, $L_{\beta}=\{\kappa \beta+\omega \zeta+n: \zeta<\kappa \wedge n \in \omega\}$ and $P_{\beta}=\{\kappa \beta+\omega \zeta+2 n: \zeta<\kappa \wedge n \in \omega\}$.

Lemma 3.29 Let $\gamma$ be a limit ordinal and let $\langle T, \triangleleft\rangle$ be a tree of height $\gamma$ such that $T=\bigcup_{\theta<\kappa} b_{\theta}$, where $b_{\theta}, \theta<\kappa$, are different branches of height $\gamma$ and let $K_{\theta}, \theta<\kappa$, be disjoint nonempty sets such that $T \cap \bigcup_{\theta<\kappa} K_{\theta}=\emptyset$. If $T_{1}=T \cup \bigcup_{\theta<\kappa} K_{\theta}$ and $\triangleleft_{1}=\triangleleft \cup \bigcup_{\theta<\kappa}\left(b_{\theta} \times K_{\theta}\right)$, then

(a) $\triangleleft=\triangleleft_{1} \cap T^{2}$;

(b) $\left\langle T_{1}, \triangleleft_{1}\right\rangle$ is a well-pruned tree of height $\gamma+1$;

(c) $\operatorname{Lev}_{\beta}\left(\left\langle T_{1}, \triangleleft 1\right\rangle\right)=\operatorname{Lev}_{\beta}(\langle T, \triangleleft\rangle)$ for all $\beta<\gamma$;

(d) $\operatorname{Lev}_{\gamma}\left(\left\langle T_{1}, \triangleleft_{1}\right\rangle\right)=\bigcup_{\theta<\kappa} K_{\theta}$. 
Lemma 3.30 Let $\kappa$ be a regular cardinal, $\mathbb{T}=\left\langle\kappa^{+}, \triangleleft\right\rangle$ a well-pruned $\kappa^{+}$tree, where $\operatorname{Lev}_{\beta}(\mathbb{T})=L_{\beta}$, for all $\beta<\kappa$ and let the functions $\Phi$ and $g$ be defined as above. Let $\gamma \in$ Ord be such that $g[\gamma] \subseteq \gamma$ and $\Phi\left[\bigcup_{\alpha<\kappa}{ }^{\alpha+1} \gamma \times 2\right] \subseteq$ $\gamma$ and let $\left\langle\beta_{\delta}^{\gamma}: \delta<\kappa\right\rangle$ be an increasing sequence in $\gamma$, cofinal in $\gamma$. If functions $\Sigma, \Sigma_{1}: \bigcup_{\alpha<\kappa}{ }^{\alpha+1} \kappa^{+} \rightarrow 2$ are such that

$$
\Phi[\Sigma] \cap \gamma=\Phi\left[\Sigma_{1}\right] \cap \gamma
$$

then $\Sigma\left(\left\langle g\left(\beta_{\delta}^{\gamma}\right): \delta \leq \alpha\right\rangle\right)=\Sigma_{1}\left(\left\langle g\left(\beta_{\delta}^{\gamma}\right): \delta \leq \alpha\right\rangle\right)$ for each $\alpha<\kappa$.

Proof. By (3.26), since $\Phi\left[\bigcup_{\alpha<\kappa}{ }^{\alpha+1} \gamma \times 2\right] \subseteq \gamma$, we have $\Phi[\Sigma] \cap \Phi\left[\bigcup_{\alpha<\kappa}{ }^{\alpha+1} \gamma \times\right.$ $2]=\Phi\left[\Sigma_{1}\right] \cap \Phi\left[\bigcup_{\alpha<\kappa}{ }^{\alpha+1} \gamma \times 2\right]$. Now, since $\Phi$ is a bijection, $\Sigma \cap \bigcup_{\alpha<\kappa}{ }^{\alpha+1} \gamma \times$ $2=\Sigma_{1} \cap \bigcup_{\alpha<\kappa}{ }^{\alpha+1} \gamma \times 2$, that is $\Sigma \uparrow \bigcup_{\alpha<\kappa}{ }^{\alpha+1} \gamma=\Sigma_{1} \uparrow \bigcup_{\alpha<\kappa}{ }^{\alpha+1} \gamma$. For each $\alpha<\kappa$ we have $\left\langle\beta_{\delta}^{\gamma}: \delta \leq \alpha\right\rangle \in{ }^{\alpha+1} \gamma$ and since $g[\gamma] \subseteq \gamma$, we have $\left\langle g\left(\beta_{\delta}^{\gamma}\right): \delta \leq \alpha\right\rangle \in{ }^{\alpha+1} \gamma$. Now, the equality of the restrictions of $\Sigma$ and $\Sigma_{1}$ to ${ }^{\alpha+1} \gamma$ gives the desired equality.

Now we are ready for the construction itself. Let $\left\langle A_{\gamma}: \gamma \in E(\kappa)\right\rangle$ be a $\nabla_{\kappa^{+}}(E(\kappa))$-sequence and let the functions $W, P, g$ and $\Phi$ be fixed as above and $T_{\alpha}=\bigcup_{\beta<\alpha} L_{\beta}$. For each $\gamma \in \operatorname{Lim} \cap \kappa^{+}$we fix an increasing sequence of successor ordinals $\left\langle\beta_{\delta}^{\gamma}: \delta<\operatorname{cf}(\gamma)\right\rangle$ cofinal in $\gamma$. We construct a tree $\mathbb{T}=\left\langle\kappa^{+}, \triangleleft_{\kappa^{+}}\right\rangle$constructing by recursion a sequence $\left\langle\triangleleft_{\alpha}: \alpha \leq \kappa^{+}\right\rangle$such that $\triangleleft_{\alpha}$ is a strict order on the set $T_{\alpha}$. The corresponding inductive property is expressed by the formula

$$
\mathcal{F}\left(\left\langle\triangleleft_{\alpha}: \alpha<\xi\right\rangle\right) \equiv \forall \alpha<\xi \quad Q\left(\alpha, \triangleleft_{\alpha},\left\langle\triangleleft_{\beta}: \beta<\alpha\right\rangle\right),
$$

where $Q\left(\alpha, \triangleleft_{\alpha},\left\langle\triangleleft_{\beta}\right.\right.$ : $\left.\left.\beta<\alpha\right\rangle\right)$ is the conjunction of the following conditions:

$\left(\mathrm{I}_{\alpha}\right)\left\langle T_{\alpha}, \triangleleft_{\alpha}\right\rangle$ is a well-pruned tree of height $\alpha$, such that $\operatorname{Lev}_{\beta}\left(\left\langle T_{\alpha}, \triangleleft_{\alpha}\right\rangle\right)=$ $L_{\beta}$, for all $\beta<\alpha$.

$\left(\mathrm{II}_{\alpha}\right) \triangleleft_{\beta}=\triangleleft_{\alpha} \cap T_{\beta}^{2}$, for all $\beta<\alpha$.

$\left(\mathrm{III}_{\alpha}\right)$ If $\alpha=\beta+2$, then

$$
\begin{aligned}
\triangleleft_{\beta+2}= & \triangleleft_{\beta+1} \cup \bigcup_{\zeta<\kappa} \bigcup_{n<\omega}(\{\kappa \beta+\omega \zeta+n\} \cup\{\kappa \beta+\omega \zeta+n\} \downarrow) \times \\
& \times\{\kappa(\beta+1)+\omega \zeta+2 n, \quad \kappa(\beta+1)+\omega \zeta+2 n+1\} .
\end{aligned}
$$

$\left(\mathrm{IV}_{\alpha}\right)$ If $\alpha=\gamma+1$ for some $\gamma \in E(\kappa)$, then (A) implies (A1), where

$$
\begin{gathered}
T_{\gamma}=\gamma \wedge A_{\gamma} \text { is a maximal antichain in }\left\langle T_{\gamma}, \triangleleft_{\gamma}\right\rangle \\
\forall t \in L_{\gamma} \exists s \in A_{\gamma} s \triangleleft_{\alpha} t .
\end{gathered}
$$


$\left(\mathrm{V}_{\alpha}\right)$ If $\alpha=\gamma+1$ for some $\gamma \in E(\kappa)$, then for every $\Sigma: \bigcup_{\alpha<\kappa}{ }^{\alpha+1} \kappa^{+} \rightarrow 2$ (B) implies (B1), where

$$
\begin{gathered}
g[\gamma] \subseteq \gamma \wedge \Phi\left[\bigcup_{\alpha<\kappa}{ }^{\alpha+1} \gamma \times 2\right] \subseteq \gamma \wedge \Phi[\Sigma] \cap \gamma=A_{\gamma} \\
\forall t \in L_{\gamma} \exists \beta<\kappa \quad \forall \delta^{\prime} \geq \beta \quad t \downarrow \cap P_{\beta_{\delta^{\prime}}^{\gamma}}^{\Sigma\left(\left\langle g\left(\beta_{\delta}^{\gamma}\right): \delta \leq \delta^{\prime}\right\rangle\right)}=\emptyset .
\end{gathered}
$$

$\left(\mathrm{VI}_{\alpha}\right)$ If $\alpha=\gamma+1$, for some $\gamma \in \kappa^{+} \cap \operatorname{Lim} \backslash E(\kappa)$, then for each branch $b$ of the tree $\mathbb{T}_{\gamma}$ such that $\operatorname{ht}(b)=\gamma$ there is $s \in L_{\gamma}$ such that $t \triangleleft_{\gamma+1} s$, for all $t \in b$.

In order to show that the recursion works, we take $\xi \leq \kappa^{+}$and assuming there is a sequence $\left\langle\triangleleft_{\alpha}: \alpha<\xi\right\rangle$ in $P\left(\kappa^{+} \times \kappa^{+}\right)$such that $\mathcal{F}\left(\left\langle\triangleleft_{\alpha}: \alpha<\xi\right\rangle\right)$, we construct $\triangleleft_{\xi} \subseteq T_{\xi}^{2}$ such that $Q\left(\xi, \triangleleft_{\xi},\left\langle\triangleleft_{\beta}: \beta<\xi\right\rangle\right)$ distinguishing the following cases:

Case 1: $\xi=0$. Then $T_{0}=\emptyset$ and $\triangleleft_{0}=\emptyset$ satisfies $\left(\mathrm{I}_{\xi}\right)-\left(\mathrm{VI}_{\xi}\right)$ trivially.

Case 2: $\xi=1$. Then $T_{1}=L_{0}=\kappa$. Let $\triangleleft_{1}=\emptyset$, then $\left\langle T_{1}, \triangleleft_{1}\right\rangle$ is an antichain and clearly $\left(\mathrm{I}_{\xi}\right)-\left(\mathrm{VI}_{\xi}\right)$ hold.

Case 3: $\xi=\beta+2$, for some $\beta<\kappa^{+}$. Since $\left(\mathrm{I}_{\beta+1}\right)$ holds, $\left\langle T_{\beta+1}, \triangleleft_{\beta+1}\right\rangle$ is a $\kappa$-sized, well-pruned tree of height $\beta+1$, hence $T_{\beta+1}=\bigcup_{\zeta<\kappa} \bigcup_{n<\omega}\{\kappa \beta+$ $\omega \zeta+n\} \cup\{\kappa \beta+\omega \zeta+n\} \downarrow$. Let $\triangleleft_{\xi}=\triangleleft_{\beta+2}$ be defined by formula (3.27). Now $\left(\mathrm{I}_{\xi}\right)$ follows from Lemma 3.29, ( $\mathrm{III}_{\xi}$ ) is provided by definition of $\triangleleft \xi$ and $\left(\mathrm{IV}_{\xi}\right),\left(\mathrm{V}_{\xi}\right)$ and $\left(\mathrm{VI}_{\xi}\right)$ are satisfied trivially. Since $\triangleleft_{\beta+1}=\triangleleft_{\beta+2} \cap T_{\beta+1}^{2}$ and $\left(\mathrm{II}_{\beta+1}\right)$ holds, we have $\left(\mathrm{II}_{\xi}\right)$.

Case 4: $\xi=\gamma+1$, for some $\gamma \in \kappa^{+} \cap \operatorname{Lim} \backslash E(\kappa)$. Let $\operatorname{cf}(\gamma)=\lambda$. We define an order $\triangleleft_{\gamma+1}$ on the set $T_{\gamma+1}=T_{\gamma} \cup\{\kappa \gamma+\theta: \theta<\kappa\}$.

First we show that each $t \in T_{\gamma}$ belongs to a branch $b \subseteq T_{\gamma}$ of height $\gamma$. Since the sequence $\left\langle\beta_{\delta}^{\gamma}: \delta<\lambda\right\rangle$ is cofinal in $\gamma$, there is $\delta_{0}<\lambda$ such that $\mathrm{ht}_{\mathbb{T}_{\gamma}}(t)<\beta_{\delta_{0}}^{\gamma}$ and, using recursion, we define the sequence $\left\langle t_{\delta}: \delta \in\left[\delta_{0}, \lambda\right)\right\rangle$ such that

(i) $t \triangleleft_{\gamma} t_{\delta_{0}}$;

(ii) $\delta^{\prime}<\delta^{\prime \prime} \Rightarrow t_{\delta^{\prime}} \triangleleft_{\gamma} t_{\delta^{\prime \prime}}$;

(iii) $t_{\delta} \in L_{\beta_{\delta}^{\gamma}}$.

By $\left(\mathrm{I}_{\gamma}\right)$, the tree $\mathbb{T}_{\gamma}$ is well-pruned, hence there is $t_{\delta_{0}} \in L_{\beta_{\delta_{0}}^{\gamma}}$ such that $t \triangleleft_{\gamma} t_{\delta_{0}}$.

Let $\delta<\lambda$ and let $t_{\eta}, \eta<\delta$, be defined such that (i)-(iii) hold. If $\delta=\eta+1$, then $t_{\eta} \in L_{\beta_{\eta}^{\gamma}}$ and, since $\mathbb{T}_{\gamma}$ is well-pruned, we can choose $t_{\eta+1} \in L_{\beta_{\eta+1}^{\gamma}}$ such that $t_{\eta} \triangleleft_{\gamma} t_{\eta+1}$. If $\delta \in \lambda \cap \operatorname{Lim}$, then $\left\langle\beta_{\eta}^{\gamma}: \eta<\delta\right\rangle$ is an increasing sequence of ordinals and for $\beta=\sup \left\{\beta_{\eta}^{\gamma}: \eta<\delta\right\}$ we have $\operatorname{cf}(\beta) \leq \operatorname{cf}(\delta) \leq \delta<\kappa$. Thus, since $\left(\mathrm{VI}_{\beta+1}\right)$ holds, there is $s \in L_{\beta}$ such that $t_{\eta} \triangleleft_{\gamma} s$, for all $\eta<\delta$. Clearly 
$\beta \leq \beta_{\delta}^{\gamma}$ and, since $\beta_{\delta}^{\gamma}$ is a successor, $\beta<\beta_{\delta}^{\gamma}$. Since $\mathbb{T}_{\gamma}$ is well-pruned, we can choose $t_{\delta} \in L_{\beta_{\delta}^{\gamma}}$ such that $s \triangleleft_{\gamma} t_{\delta}$.

Now, the branch $b_{t}$ containing the chain $\left\{t_{\delta}: \delta \in\left[\delta_{0}, \lambda\right)\right\}$ is of height $\gamma$ and contains $t$.

Since the tree $\mathbb{T}_{\gamma}$ contains antichains of size $\kappa$, we have $|B| \geq \kappa$, where $B=\left\{b \subseteq T_{\gamma}: b\right.$ is a branch of height $\left.\gamma\right\}$. On the other hand, the function $f: B \rightarrow^{\lambda}\left(\left[T_{\gamma}\right]^{1}\right)$ defined by $f(b)=\left\langle b \cap L_{\beta_{\delta}^{\gamma}}: \delta<\lambda\right\rangle$ is an injection and, since $\left|T_{\gamma}\right|=\kappa$, we have $|B| \leq \kappa^{\lambda} \leq \kappa^{<\kappa}=\kappa$ and, consequently, $|B|=\kappa$.

Let $\left\{b_{\theta}: \theta<\kappa\right\}$ be an enumeration of $B$. Then, as we proved above, $T_{\gamma}=\bigcup_{\theta<\kappa} b_{\theta}$ and we define the order on the set $T_{\xi}=T_{\gamma} \cup L_{\gamma}$ by $\triangleleft_{\xi}=\triangleleft_{\gamma}$ $\cup \bigcup_{\theta<\kappa}\left(b_{\theta} \times\{\kappa \gamma+\theta\}\right)$. Now, since $\left(\mathrm{II}_{\gamma}\right)$ holds, according to Lemma 3.29, we have $\left(\mathrm{I}_{\xi}\right)$ and $\left(\mathrm{II}_{\xi}\right)$. Conditions $\left(\mathrm{III}_{\xi}\right),\left(\mathrm{IV}_{\xi}\right)$ and $\left(\mathrm{V}_{\xi}\right)$ are satisfied trivially and $\left(\mathrm{VI}_{\xi}\right)$ follows from the construction.

Case 5: $\xi=\gamma+1$ for some $\gamma \in E(\kappa)$. We define an order $\triangleleft_{\gamma+1}$ on the set $T_{\gamma+1}=T_{\gamma} \cup\{\kappa \gamma+\theta: \theta<\kappa\}$. Using $\left(\mathrm{I}_{\gamma}\right)$ we will choose different branches of height $\gamma, b_{\theta}, \theta<\kappa$, in $T_{\gamma}$ such that $T_{\gamma}=\bigcup_{\theta<\kappa} b_{\theta}$ and define $\triangleleft_{\gamma+1}=\triangleleft_{\gamma} \cup \bigcup_{\theta<\kappa}\left(b_{\theta} \times\{\kappa \gamma+\theta\}\right)$. Then, by Lemma 3.29, condition ( $\left.\mathrm{I}_{\xi}\right)$ will be satisfied. Condition ( $\mathrm{II}_{\xi}$ ) will hold since $\triangleleft_{\gamma}=\triangleleft_{\gamma+1} \cap T_{\gamma}^{2}$ and ( $\mathrm{II}_{\gamma}$ ) is true by the assumption. Thus, since conditions $\left(\mathrm{III}_{\xi}\right)$ and $\left(\mathrm{VI}_{\xi}\right)$ will be satisfied trivially, only conditions $\left(\mathrm{IV}_{\xi}\right)$ and $\left(\mathrm{V}_{\xi}\right)$ will remain for verification. Depending of whether conditions (A) and (B) are satisfied, we distinguish the following four subcases.

Subcase 5.1: (A) holds and there is $\Sigma: \bigcup_{\alpha<\kappa}{ }^{\alpha+1} \kappa^{+} \rightarrow 2$ such that (B) holds. By Lemma 3.30 it suffices to prove (B1) for that $\sigma$.

Let $x \in T_{\gamma}$. Since $A_{\gamma}$ is a maximal antichain in $\mathbb{T}_{\gamma}$, there exists $s \in A_{\gamma}$ comparable with $x$ and we define $y=\max \{x, s\}$. Then $\operatorname{ht}_{\mathbb{T}_{\gamma}}(y)<\gamma$, so there is $\delta_{0}<\kappa$ such that $\operatorname{ht}_{\mathbb{T}_{\gamma}}(y)<\beta_{\delta_{0}}^{\gamma}$. Using recursion we define a sequence $\left\langle y_{\delta}: \delta \in\left[\delta_{0}, \kappa\right)\right\rangle$ such that

(i) $y \triangleleft_{\gamma} y_{\delta_{0}}$;

(ii) $\delta^{\prime}<\delta^{\prime \prime} \Rightarrow y_{\delta^{\prime}} \triangleleft_{\gamma} y_{\delta^{\prime \prime}}$;

(iii) $y_{\delta^{\prime}} \in L_{\beta_{\delta^{\prime}}^{\gamma}} \backslash P_{\beta_{\delta^{\prime}}^{\gamma}}^{\Sigma\left(\left\langle g\left(\beta_{\delta}^{\gamma}\right): \delta \leq \delta^{\prime}\right\rangle\right)}$.

Since the tree $\mathbb{T}_{\gamma}$ is well-pruned, there is $z \in L_{\beta_{\delta_{0}}^{\gamma}-1}$ such that $y \unlhd_{\gamma} z$ and since $\left(\mathrm{III}_{\beta_{\delta_{0}}^{\gamma}+1}\right)$ holds, $z$ has both an even and an odd successor on the level $L_{\beta_{\delta_{0}}^{\gamma}}$. Now we choose the successor $y_{\delta_{0}}$ of $z$ such that $y_{\delta_{0}} \in L_{\beta_{\delta_{0}}^{\gamma}}$ । $P_{\beta_{\delta_{0}}^{\gamma}}^{\Sigma\left(\left\langle g\left(\beta_{\delta}^{\gamma}\right): \delta \leq \delta_{0}\right\rangle\right)}$.

Let $\delta^{\prime}<\kappa$ and let the sequence $\left\langle y_{\delta}: \delta \in\left[\delta_{0}, \delta^{\prime}\right)\right\rangle$ satisfy (i)-(iii). 
If $\delta^{\prime}=\eta+1$, since the tree $\mathbb{T}_{\gamma}$ is well-pruned, there is $z \in L_{\beta_{\delta^{\prime}}^{\gamma}-1}$ such that $y_{\eta} \unlhd_{\gamma} z$ and, since ( $\operatorname{III}_{\beta_{\delta^{\prime}}^{\gamma}+1}$ ) holds, $z$ has both an even and an odd successor on the level $L_{\beta_{\delta^{\prime}}^{\gamma}}$. Now we choose the successor $y_{\delta^{\prime}}$ of $z$ such that $y_{\delta^{\prime}} \in L_{\beta_{\delta^{\prime}}^{\gamma}} \backslash P_{\beta_{\delta^{\prime}}^{\gamma}}^{\Sigma\left(\left\langle g\left(\beta_{\delta}^{\gamma}\right): \delta \leq \delta^{\prime}\right\rangle\right)}$, and the sequence $\left\langle y_{\delta}: \delta \in\left[\delta_{0}, \delta^{\prime}\right]\right\rangle$ satisfies (i)-(iii).

If $\delta^{\prime} \in \operatorname{Lim}$ and $\beta=\sup \left\{\beta_{\delta}^{\gamma}: \delta<\delta^{\prime}\right\}$, then $\operatorname{cf}(\beta) \leq \delta^{\prime}<\kappa$. Since $\left\{y_{\delta}: \delta \in\left[\delta_{0}, \delta^{\prime}\right)\right\}$ is an increasing chain and $y_{\delta} \in L_{\beta_{\delta}^{\gamma}}$, there is a branch $b$ of height $\beta$ in $\mathbb{T}_{\beta}$ containing $y_{\delta}, \delta \in\left[\delta_{0}, \delta^{\prime}\right)$, thus, by $\left(\mathrm{VI}_{\beta+1}\right)$, there is $t \in L_{\beta}$ such that $y_{\delta} \triangleleft_{\gamma} t$, for all $\delta<\delta^{\prime}$. Clearly $\beta \leq \beta_{\delta^{\prime}}^{\gamma}$, but, since $\beta_{\delta^{\prime}}^{\gamma}$ is a successor ordinal, we have $\beta \leq \beta_{\delta^{\prime}}^{\gamma}-1$. Now we take $z \in L_{\beta_{\delta^{\prime}}^{\gamma}-1}$ satisfying $t \unlhd_{\gamma} z$ and define $y_{\delta^{\prime}}$ as in the previous cases. So, the sequence $\left\langle y_{\delta}: \delta \in\left[\delta_{0}, \kappa\right)\right\rangle$ satisfying (i)-(iii) is defined.

Let $b_{x}$ be the branch in $\mathbb{T}_{\gamma}$ such that $y_{\delta} \in b_{x}$ for all $\delta \in\left[\delta_{0}, \kappa\right)$. In this way to each $x \in T_{\gamma}$ we adjoin a branch $b_{x} \subseteq T_{\gamma}$ of height $\gamma$ and $T_{\gamma}=\bigcup_{x \in T_{\gamma}} b_{x}$. Since $\left|T_{\gamma}\right|=\kappa$, there is an 1-1 enumeration $\left\{b_{x}: x \in T_{\gamma}\right\}=\left\{b_{\theta}: \theta<\kappa\right\}$. Then $T_{\gamma}=\bigcup_{\theta<\kappa} b_{\theta}$ and we define the order $\triangleleft_{\xi}=\triangleleft_{\gamma+1}$ on $T_{\gamma} \cup L_{\gamma}$ by

$$
\triangleleft_{\gamma+1}=\triangleleft_{\gamma} \cup \bigcup_{\theta<\kappa}\left(b_{\theta} \times\{\kappa \gamma+\theta\}\right) .
$$

For the proof of $\left(\mathrm{IV}_{\xi}\right)$ and $\left(\mathrm{V}_{\xi}\right)$ we check (A1) and (B1). Let $t=\kappa \gamma+\theta \in L_{\gamma}$. Then in the tree $\left\langle T_{\gamma+1}, \triangleleft_{\gamma+1}\right\rangle$ we have $t \downarrow=b_{\theta}$. By the construction, the branch $b_{\theta}$ contains an element $s$ of $A_{\gamma}$. Clearly $s \triangleleft_{\xi} t$ and (A1) is proved. Also there exists $\delta_{0}<\kappa$ such that $b_{\theta} \cap L_{\beta_{\delta^{\prime}}^{\gamma}} \subseteq L_{\beta_{\delta^{\prime}}^{\gamma}} \backslash P_{\beta_{\delta^{\prime}}^{\gamma}}^{\Sigma\left(\left\langle g\left(\beta_{\delta}^{\gamma}\right): \delta \leq \delta^{\prime}\right\rangle\right)}$ is satisfied for each $\delta^{\prime} \in\left[\delta_{0}, \kappa\right)$ so (B1) holds.

The other three subcases are similar to 5.1. Namely, in the construction of the branch $b_{x}$ (for $x \in T_{\gamma}$ ) if $\neg(\mathrm{A}) \wedge \exists \Sigma(\mathrm{B})$, we start from $y=x$ (ignoring $\left.A_{\gamma}\right)$; if $(\mathrm{A}) \wedge \neg \exists \Sigma(\mathrm{B})$, then we choose arbitrary $y_{\delta} \in L_{\beta_{\delta}^{\gamma}}$ for $\delta \geq \delta_{0}$ such that the sequence $\left\langle y_{\delta}\right\rangle$ is increasing and, finally, if $\neg(\mathrm{A}) \wedge \neg \exists \Sigma(\mathrm{B})$ then we start from $y=x$ and choose $y_{\delta}$ like in subcase $(\mathrm{A}) \wedge \neg(\mathrm{B})$.

Case 6: $\xi \in \mathrm{Lim}$. (The case $\xi=\kappa^{+}$is included here.) Let the order $\triangleleft_{\xi}$ on $T_{\xi}=\bigcup_{\beta<\xi} L_{\beta}=\bigcup_{\alpha<\xi} T_{\alpha}$ be defined by $\triangleleft_{\xi}=\bigcup_{\alpha<\xi} \triangleleft_{\alpha}$. Then (III Th ), $\left(\mathrm{IV}_{\xi}\right),\left(\mathrm{V}_{\xi}\right)$ and $\left(\mathrm{VI}_{\xi}\right)$ hold trivially. By the assumption $\beta \leq \alpha<\xi$ implies $\triangleleft_{\beta}=\triangleleft_{\alpha} \cap T_{\beta}^{2}$, thus for $\beta<\xi$, since the sequence $\left\langle\triangleleft_{\alpha}: \alpha<\xi\right\rangle$ is increasing, we have $\triangleleft_{\xi} \cap T_{\beta}^{2}=\bigcup_{\beta \leq \alpha<\xi} \triangleleft_{\alpha} \cap T_{\beta}^{2}=\triangleleft_{\beta}$ and $\left(\mathrm{II}_{\xi}\right)$ is proved. Consequently, $\left\langle T_{\xi}, \triangleleft_{\xi}\right\rangle$ is a strict order since it is the union of the chain of strict orders $\left\langle T_{\alpha}, \triangleleft_{\alpha}\right\rangle, \alpha<\xi$. It is easy to check that for each $\beta<\xi$ and each $t \in L_{\beta}$

$$
\begin{aligned}
\operatorname{pred}_{\left\langle T_{\xi}, \triangleleft_{\xi}\right\rangle}(t) & =\operatorname{pred}_{\left\langle T_{\beta+1}, \triangleleft_{\beta+1}\right\rangle}(t) \\
\triangleleft_{\xi} \cap \operatorname{pred}_{\left\langle T_{\xi}, \triangleleft_{\xi}\right\rangle}(t)^{2} & =\triangleleft_{\beta+1} \cap \operatorname{pred}_{\left\langle T_{\beta+1}, \triangleleft_{\beta+1}\right\rangle}(t)^{2}
\end{aligned}
$$


so, by the assumption $\left(\mathrm{I}_{\beta+1}\right)$, the set $\operatorname{pred}_{\left\langle T_{\xi}, \triangleleft_{\xi}\right\rangle}(t)$ is well-ordered by $\triangleleft_{\xi}$, thus $\left\langle T_{\xi}, \triangleleft_{\xi}\right\rangle$ is a tree. By (3.28), (3.29) and $\left(\mathrm{I}_{\beta+1}\right)$ we have

$$
\left.\mathrm{ht}_{\left\langle T_{\xi}, \triangleleft \xi\right.}(t)=\mathrm{ht}_{\left\langle T_{\beta+1}, \triangleleft \beta+1\right.}\right\rangle=\beta,
$$

thus $\operatorname{Lev}_{\beta}\left(\mathbb{T}_{\xi}, \triangleleft_{\xi}\right)=L_{\beta}$ for all $\beta<\xi$. Since $T_{\xi}=\bigcup_{\beta<\xi} L_{\beta}$, the tree $\left\langle T_{\xi}, \triangleleft_{\xi}\right\rangle$ is of height $\xi$. If $\alpha<\beta<\xi$ and $s \in L_{\alpha}$, then $s \in T_{\beta+1}=T_{\beta} \cup L_{\beta}$ so, by $\left(\mathrm{I}_{\beta+1}\right)$, there is $t \in L_{\beta}$ such that $s \triangleleft_{\beta+1} t$, which implies $s \triangleleft_{\xi} t$. Thus the tree $\left\langle T_{\xi}, \triangleleft_{\xi}\right\rangle$ is well-pruned and $\left(\mathrm{I}_{\xi}\right)$ holds.

According to the Recursion Principle there is a sequence $\left\langle\triangleleft_{\alpha}: \alpha \leq \kappa^{+}\right\rangle$ satisfying $\mathcal{F}$. It is easy to show that $T_{\kappa^{+}}=\kappa^{+}$. Since $\left(\mathrm{I}_{\kappa^{+}}\right)$holds, $\mathbb{T}=$ $\left\langle\kappa^{+}, \triangleleft_{\kappa^{+}}\right\rangle$is a well-pruned tree of height $\kappa^{+}$such that $\operatorname{Lev}_{\beta}(\mathbb{T})=L_{\beta}$, for all $\beta<\kappa^{+}$. Consequently $\mathbb{T}$ is a well-pruned $\kappa^{+}$-tree. $\mathbb{T}$ is also ever-branching: let $t=\kappa \beta+\omega \zeta+n \in L_{\beta}$ and $\alpha=\beta+2$. ( III $\left._{\alpha}\right)$ implies that in the tree $\mathbb{T}_{\beta+2} t$ has two incomparable successors and by $\left(\mathrm{II}_{\kappa^{+}}\right)$this holds in $\mathbb{T}$ as well.

The following two lemmas are generalizations of Lemmas II 7.6 and II 7.7 from [24].

Lemma 3.31 Let $\mathbb{T}=\left\langle\kappa^{+}, \triangleleft\right\rangle$ be a $\kappa^{+}$-tree and $A$ a maximal antichain in $\mathbb{T}$. Then the set $C_{A}=\left\{\gamma<\kappa^{+}: T_{\gamma}=\gamma \wedge A \cap T_{\gamma}\right.$ is a maximal antichain in $\left.T_{\gamma}\right\}$ contains a club subset of $\kappa^{+}$.

Proof. We define functions $f, g, h: \kappa^{+} \rightarrow \kappa^{+}$as follows: $f(\xi)=h t(\xi)$, $g(\xi)=\sup L_{\xi}$ and $h(\xi)=$ the element of antichain $A$ comparable to $\xi$. Now the sets $C_{f}=\left\{\xi<\kappa^{+}: \xi \subseteq T_{\xi}\right\}, C_{g}=\left\{\xi<\kappa^{+}: T_{\xi} \subseteq \xi\right\}$ and $C_{h}=\{\xi<$ $\kappa^{+}: A \cap T_{\xi}$ is a maximal antichain in $\left.\mathbb{T}_{\xi}\right\}$ are clubs in $\kappa^{+}$by Lemma 1.4, so by Lemma $1.5 C_{A}=C_{f} \cap C_{g} \cap C_{h}$ is a club in $\kappa^{+}$as well.

Lemma 3.32. Let $\kappa$ be a regular cardinal satisfying $\nabla_{\kappa^{+}}(E(\kappa))$, let $\left\langle A_{\gamma}\right.$ : $\gamma \in E(\kappa)\rangle$ be a $\nabla_{\kappa^{+}}(E(\kappa))$-sequence and let $\mathbb{T}=\left\langle\kappa^{+}, \triangleleft\right\rangle$ be an everbranching $\kappa^{+}$-tree such that for each $\gamma \in E(\kappa)$ (A) implies (A1), where

$$
\begin{gathered}
T_{\gamma}=\gamma \wedge A_{\gamma} \text { is a maximal antichain in } \mathbb{T}_{\gamma} \\
\forall s \in \operatorname{Lev}_{\gamma}(\mathbb{T}) \quad \exists a \in A_{\gamma} a \triangleleft s .
\end{gathered}
$$

then $\mathbb{T}$ is a $\kappa^{+}$-Suslin tree.

Proof. Let $A \subseteq \kappa^{+}$be a maximal antichain in $\mathbb{T}$. Then $S=\{\gamma \in E(\kappa): A \cap$ $\left.\gamma=A_{\gamma}\right\}$ is a stationary subset of $\kappa^{+}$so, by Lemma 3.31, there is $\gamma \in S \cap C_{A}$. Now $A \cap T_{\gamma}=A \cap \gamma=A_{\gamma}$ so $\gamma$ satisfies (A) and, by the assumption, (A1) 
as well. Let $t \in T$ and $h t(t) \geq \gamma$. Then there is $s \in \operatorname{Lev}_{\gamma}(\mathbb{T})$ such that $s \triangleleft t$ so, by (A1), there is $a \in A_{\gamma}=A \cap \gamma$ such that $a \triangleleft s$. This implies $a \triangleleft t$ and therefore $t \notin A$. Thus $A \subseteq T_{\gamma}$ and $A=A \cap T_{\gamma}=A \cap \gamma=A_{\gamma}$, which implies $|A| \leq \kappa$

Suppose $B$ is a $\kappa^{+}$-sized branch of $\mathbb{T}$. Using recursion we define a sequence $\left\langle a_{\alpha}: \alpha<\kappa^{+}\right\rangle$of incomparable elements of $T \backslash B$. Let $\alpha<\kappa^{+}$and suppose $a_{\beta}$ are defined for $\beta<\alpha$. Then $\xi_{\alpha}=\sup \left\{\operatorname{ht}\left(a_{\beta}\right): \beta<\alpha\right\}<\kappa^{+}$ and we choose $b_{\alpha} \in B$ such that $\operatorname{ht}\left(b_{\alpha}\right)>\xi_{\alpha}$. Since the tree $\mathbb{T}$ is everbranching we can choose $a_{\alpha} \in T \backslash B$ such that $b_{\alpha} \triangleleft a_{\alpha}$. Clearly, $a_{\alpha}$ and $a_{\beta}$ are incomparable, for each $\beta<\alpha$.

Now $\left\{a_{\alpha}: \alpha<\kappa^{+}\right\}$is an antichain in $\mathbb{T}$ of cardinality $\kappa^{+}$. Since we previously showed that such antichain can not exist, this is a contradiction.

In order to apply Lemma 3.32 we suppose $\gamma \in E(\kappa)$ satisfies $(\mathrm{A})$. Then $\left(\mathrm{IV}_{\alpha}\right)$ holds for $\alpha=\gamma+1$, so (A) implies (A1), that is for each $t \in L_{\gamma}$ there is $s \in A_{\gamma}$ satisfying $s \triangleleft_{\alpha} t$ which, by ( $\mathrm{II}_{\kappa^{+}}$), implies $s \triangleleft_{\kappa^{+}} t$. Thus all the assumptions of Lemma 3.32 are satisfied, $\mathbb{T}$ is a well-pruned $\kappa^{+}$-Suslin tree and, by Lemma 1.80, White does not have a winning strategy in the game $\mathcal{G}_{\mathrm{ls}}(\kappa)$ played on $\mathbb{B}$.

Now we isolate from the construction above the property which ensures that Black does not have a winning strategy in the game $\mathcal{G}_{\mathrm{ls}}^{\prime \prime}(\kappa)$.

Theorem 3.33 Let $\kappa$ be a regular cardinal and for each $\gamma \in E(\kappa)$ let $\left\langle\beta_{\delta}^{\gamma}: \delta<\kappa\right\rangle$ be an increasing sequence in $\gamma$, cofinal in $\gamma$. Let $\nabla_{\kappa^{+}}(E(\kappa))$ hold and let $\left\langle A_{\gamma}: \gamma \in E(\kappa)\right\rangle$ be a $\nabla_{\kappa^{+}}(E(\kappa))$-sequence. Let $\mathbb{T}=\left\langle\kappa^{+}, \triangleleft\right\rangle$ be a well-pruned $\kappa^{+}$-tree, where $\operatorname{Lev}_{\beta}(\mathbb{T})=L_{\beta}$, for all $\beta<\kappa$ and let the functions $W, \Phi, P$ and $g$ be defined as above. If for every $\gamma \in E(\kappa)$ and every $\Sigma: \bigcup_{\alpha<\kappa}{ }^{\alpha+1} \kappa^{+} \rightarrow 2$

$$
g[\gamma] \subseteq \gamma \wedge \Phi\left[\bigcup_{\alpha<\kappa}{ }^{\alpha+1} \gamma \times 2\right] \subseteq \gamma \wedge \Phi[\Sigma] \cap \gamma=A_{\gamma}
$$

implies that

$$
\forall t \in L_{\gamma} \exists \beta<\kappa \forall \alpha \geq \beta \quad t \downarrow \cap P_{\beta_{\alpha}^{\gamma}}^{\Sigma\left(\left\langle g\left(\beta_{\delta}^{\gamma}\right): \delta \leq \alpha\right\rangle\right)}=\emptyset,
$$

then Black does not have a winning strategy in the game $\mathcal{G}_{\mathrm{ls}}^{\prime \prime}(\kappa)$ played on the algebra $\mathbb{B}=$ r.o. $\left(\mathbb{T}^{*}\right)$.

Proof. Let $\Sigma$ be a strategy for Black in the game $\mathcal{G}_{\mathrm{ls}}^{\prime \prime}(\kappa)$ on the algebra $\mathbb{B}$ and let $\bar{\Sigma}: \bigcup_{\alpha<\kappa}{ }^{\alpha+1} \kappa^{+} \rightarrow 2$ be the corresponding function defined by 
(3.24). Then $\Phi[\bar{\Sigma}] \subseteq \kappa^{+}$and, by $\diamond_{\kappa^{+}}(E(\kappa))$, the set $S=\{\gamma \in E(\kappa)$ : $\left.\Phi[\bar{\Sigma}] \cap \gamma=A_{\gamma}\right\}$ is a stationary subset of $\kappa^{+}$. According to Lemmas 3.26 and 3.27 the sets $C_{P}=\left\{\gamma<\kappa^{+}: P[\gamma] \subseteq W[\gamma]\right\}=\left\{\gamma<\kappa^{+}: g[\gamma] \subseteq \gamma\right\}$ and $C_{\Phi}=\left\{\gamma<\kappa^{+}: \Phi\left[\bigcup_{\alpha<\kappa}{ }^{\alpha+1} \gamma \times 2\right] \subseteq \gamma\right\}$ are club subsets of $\kappa^{+}$and, hence, there exists $\gamma \in C_{P} \cap C_{\Phi} \cap S$. This implies $g[\gamma] \subseteq \gamma, \Phi\left[\bigcup_{\alpha<\kappa}{ }^{\alpha+1} \gamma \times 2\right] \subseteq \gamma$ and $\Phi[\bar{\Sigma}] \cap \gamma=A_{\gamma}$ thus (B) holds. By the assumption (B1) holds as well.

Let us consider the play in which White plays $\left\langle\bigvee P_{\beta_{\delta}^{\gamma}}: \delta<\kappa\right\rangle$ and Black follows $\Sigma$. According to (3.25) in the $\alpha$-th move, when the sequence of White's previous moves is $\left\langle\bigvee P_{\beta_{\delta}^{\gamma}}: \delta \leq \alpha\right\rangle$, Black chooses $\bigvee P_{\beta_{\alpha}^{\gamma}}^{\bar{\Sigma}\left(\left\langle g\left(\beta_{\delta}^{\gamma}\right): \delta \leq \alpha\right\rangle\right)}$. Suppose Black wins the play, that is

$$
\bigwedge_{\beta<\kappa} \bigvee_{\alpha \geq \beta} \bigvee P_{\beta_{\alpha}^{\gamma}}^{\bar{\Sigma}\left(\left\langle g\left(\beta_{\delta}^{\gamma}\right): \delta \leq \alpha\right\rangle\right)}=b>0
$$

Let $\leq$ denote the corresponding order on the algebra $\mathbb{B}=$ r.o. $\left(\mathbb{T}^{*}\right)$. Then, because of (3.30) there is $s \in T$ such that $s \leq b$ and, since the tree is wellpruned, there is $v \in T$ satisfying $v \leq s$ and $\operatorname{ht}(v) \geq \gamma$. Let $t \in L_{\gamma}$ be such that $v \leq t$. Since $v \leq b$, by (3.30) for each $\beta<\kappa$ there are $\alpha \geq \beta$ and $u \in P_{\beta_{\alpha}^{\gamma}}^{\bar{\Sigma}\left(\left\langle g\left(\beta_{\delta}^{\gamma}\right): \delta \leq \alpha\right\rangle\right)}$ such that $u \wedge v>0$ which implies $u \wedge t>0$. But this means that $u$ and $t$ are comparable elements of $T$, thus, since ht $(u)=\beta_{\alpha}^{\gamma}<$ $\gamma=\operatorname{ht}(t)$, we have $u \triangleleft t$, which implies $u \in t \downarrow \cap P_{\beta_{\alpha}^{\gamma}}^{\bar{\Sigma}\left(\left\langle g\left(\beta_{\delta}^{\gamma}\right): \delta \leq \alpha\right\rangle\right)}$. Thus

$$
\exists t \in L_{\gamma} \forall \beta<\kappa \quad \exists \alpha \geq \beta \quad t \downarrow \cap P_{\beta_{\alpha}^{\gamma}}^{\bar{\Sigma}\left(\left\langle g\left(\beta_{\delta}^{\gamma}\right): \delta \leq \alpha\right\rangle\right)} \neq \emptyset .
$$

A contradiction, since $\bar{\Sigma}$ satisfies (B1). So White wins the play and $\Sigma$ is not a winning strategy for Black.

In order to apply Theorem 3.33 suppose $\gamma \in E(\kappa)$ satisfies condition (B). Then $\alpha=\gamma+1<\kappa^{+}$. Condition $\left(\mathrm{V}_{\alpha}\right)$ holds so, because of $(\mathrm{B})$,

$$
\forall t \in L_{\gamma} \quad \exists \beta<\kappa \quad \forall \delta^{\prime} \geq \beta \quad t \downarrow \cap P_{\beta_{\delta^{\prime}}^{\gamma}}^{\Sigma\left(\left\langle g\left(\beta_{\delta}^{\gamma}\right): \delta \leq \delta^{\prime}\right\rangle\right)}=\emptyset .
$$

So all the assumptions of Theorem 3.33 are satisfied and Black does not have a winning strategy in the game $\mathcal{G}_{\mathrm{ls}}^{\prime \prime}(\kappa)$ played on $\mathbb{B}=$ r.o. $\left(\mathbb{T}^{*}\right)$. This concludes the proof of Theorem 3.23.

The question remains: is there a ZFC example of a Boolean algebra on which the game is undetermined, or at least: can the assumption $\diamond_{\kappa^{+}}(E(\kappa))$ be weakened to $\nabla_{\kappa^{+}}$? Perhaps this could be done by modifying the example of a Boolean algebra on which $\mathcal{G}_{\text {dist }}(\omega, 2,2)$ is undetermined, described in [38]. 



\section{Bibliography}

[1] U. Abraham, S. Todorčević, Partition properties of $\omega_{1}$ compatible with CH, Fund. Math. 152 (2) (1997) 165-181.

[2] B. Balcar, T. Jech, T. Pazák, Complete ccc Boolean algebras, the order sequential topology and a problem of von Neumann, Bull. Lond.q Math. Soc. 37 (6) (2005) 885-898.

[3] J. Baumgartner, Iterated Forcing, in Surveys in Set Theory (A. R. D. Mathias, ed.), London Math. Soc. Lecture Note Ser. 87, Cambridge University Press, Cambridge-New York, 1983, 1-59.

[4] J. Baumgartner, L. Harrington, E. Kleinberg, Adding a closed unbounded set, J. Symbolic Logic 41 (1976), 481-482.

[5] J. L. Bell, Boolean-valued models and independence proofs, 3rd edition, Oxford University Press, New York, 2005.

[6] P. J. Cohen, The independence of the continuum hypothesis, Proc. Natl. Acad. Sci. USA 50 (1963), 1143-1148.

[7] P. J. Cohen, The independence of the continuum hypothesis II, Proc. Natl. Acad. Sci. USA 51 (1964), 105-110.

[8] N. Dobrinen, Generalized weak distributive laws in Boolean algebras and issues related to a problem of von Neumann regarding measurable algebras, PhD thesis, The University of Minnesota, 2001.

[9] N. Dobrinen, Games and generalized distributive laws in Boolean algebras, Proc. Amer. Math. Soc. 131 (1) (2003) 309-318.

[10] N. Dobrinen, Errata to "Games and generalized distributive laws in Boolean algebras", Proc. Amer. Math. Soc. 131 (9) (2003) 2967-2968. 
[11] I. Farah, J. Zapletal, Between Maharam's and von Neumann's problems, Math. Res. Lett. 11 (5-6) (2004) 673-684.

[12] M. Foreman, Games played on Boolean algebras, J. Symbolic Logic 48 (1983), 714-723.

[13] S. Fuchino, H. Mildenberger, S. Shelah, P. Vojtás, On absolutely divergent series, Fund. Math. 160 (1999), 255-268.

[14] T. Jech, The Axiom of Choice, North Holland, Amsterdam-London, 1973.

[15] T. Jech, A game theoretic property of Boolean algebras, in Logic Colloquium '77 (A. Macintyre, L. Pacholski, J. Paris, eds.), North-Holland, Amsterdam-London, 1978, 135-144.

[16] T. Jech, More game-theoretic properties of Boolean algebras, Ann. Pure Appl. Logic 26 (1984) 11-29.

[17] T. Jech, Distributive laws, in Handbook of Boolean algebras (J. D. Monk, ed.), North-Holland, Amsterdam-London, 1989, 317-331.

[18] T. Jech, Multiple Forcing, Cambridge Tracts in Mathematics, Vol. 88.

[19] T. Jech, Set Theory, the Third Millenium Edition, Springer-Verlag, Berlin-New York, 2002.

[20] T. Jech, S. Shelah, On countably closed complete Boolean algebras, J. Symbolic Logic 61 (1996), 1380-1386.

[21] M. Kada, More on Cichon's diagram and infinite games, J. Symbolic Logic 65 (2000), 1713-1724.

[22] A. Kamburelis, On the weak distributivity game, Ann. Pure Appl. Logic 66 (1994), 19-26.

[23] A. Kanamori, The Higher Infinite, Second Edition, Springer-Verlag, Berlin-New York, 2005.

[24] K. Kunen, Set Theory, An Introduction to Independence Proofs, NorthHolland, Amsterdam-London, 1980.

[25] M. S. Kurilić, B. Šobot, A game on Boolean algebras describing the collapse of the continuum, to appear in Ann. Pure Appl. Logic. 
[26] M. S. Kurilić, B. Šobot, Power collapsing games, J. Symbolic Logic 73 (2008), no. 4 1433-1457.

[27] D. A. Martin, R. M. Solovay, Internal Cohen extensions, Ann. Math. Logic 2 (1970), no. 2 143-178.

[28] K. Prikry, Changing measurable into accessible cardinals, Dissertationes Mathematicae 68 (1971), 359-378.

[29] G. E. Sacks, Forcing with perfect closed sets, in Axiomatic Set Theory, Proc. Sympos. Pure Math. Los Angeles 1967, Amer. Math. Soc. Providence, R.I. 1971, 331-355.

[30] R. Sikorski, Boolean Algebras, 3rd edition, Springer-Verlag, Berlin-New York, 1969.

[31] R. M. Solovay, A model of set theory in which every set is Lebesgue measurable, Ann. of Math. (2) 92 (1970), 1-56.

[32] R. M. Solovay, S. Tennenbaum, Iterated Cohen extensions and Souslin's problem, Ann. of Math. (2) 94 (1971), 201-245.

[33] S. Todorčević, Trees, subtrees and order types, Ann. Math. Logic 20 (1981), 233-268.

[34] S. Todorčević, Trees and linear orderings, in Handbook of Set-Theoretic Topology (K. Kunen, J. Vaughan, eds.), North-Holland, AmsterdamLondon, 1988.

[35] S. Todorčević, A dichotomy for P-ideals of countable sets, Fund. Math. 166 (3) (2000) 251-267.

[36] B. Veličković, Playful Boolean algebras, Trans. Amer. Math. Soc. 296 (1986) 727-740.

[37] B. Veličković, ccc forcing and splitting reals, Israel J. Math. 147 (2005) 209-220.

[38] J. Zapletal, More on the cut and choose game, Ann. Pure Appl. Logic 76 (1995) 291-301. 


\section{Index}

antichain, 10

maximal, 10

$\mathcal{A}(\mathbb{P}), 61$

$\mathcal{A}_{p}, 47$

$\mathcal{A}_{p}(\lambda), 47$

$\mathcal{A}_{p}(\leq \lambda), 47$

ascending function, 48

Axiom of Determinacy, 41

$\operatorname{Black}(\mathbb{B}), 86$

Boolean algebra

atomless, 16

Cohen algebra, 35

complete, 9

$\kappa$-distributive, 26

$(\kappa, \lambda)$-distributive, 26

$(\kappa, \lambda, \mu)$-distributive, 31

Maharam algebra, 85

weakly distributive, 31

weakly $(\kappa, \lambda)$-distributive, 31

Boolean-valued model, 13

branch, 37

c.c.c., 10

c.c. $(\mathbb{P}), 10$

chain condition, 10

club, 6

cofinality, $\mathrm{cf}(\kappa), 6$

$\operatorname{Col}(\kappa, \lambda), 35$

completion, 9

diamond principle

$\diamond, 7$ $\nabla_{\kappa}, 7$

$D\left({ }^{n} P\right), 42$

embedding

canonical, 9

complete, 21

dense, 21

filter, 11

$\mathbb{P}$-generic, 12

forcing

Cohen, 34

collapse, 35

Prikry, 36

random, 35

shooting a club, 35

forcing conditions, 12

forcing relation, $\Vdash, 18$

formula

absolute, 15

game

Banach-Mazur game, $\mathcal{G}_{\mathrm{BM}}, 39$

cut-and-choose, 47

descending chain game, $\mathcal{G}_{\text {inf }}(\kappa)$, 47

descending chain game, $\mathcal{G}_{\text {inf }}(\omega)$, 42

distributivity game, $\mathcal{G}_{\text {dist }}, 54$

distributivity game $\mathcal{G}_{\text {dist }}^{\prime}, 65$

$\mathcal{G}_{\mathrm{b}}, 72$

$\mathcal{G}_{\mathrm{lp}}, 73$

$\mathcal{G}_{\mathrm{ls}}, 75$ 
$\mathcal{G}_{\mathrm{ls}}^{\prime \prime}, 90$

$\mathcal{G}^{\prime}, 41$

$\mathcal{G}_{\mathrm{sp}}, 69$

undetermined, 40

$\mathfrak{h}_{2}(\mathbb{B}), 26$

ht $(\mathbb{T}), 36$

$\mathrm{ht}_{\mathbb{T}}(t), 36$

incompatible

elements, 8

sets, 9

$\operatorname{Lev}_{\alpha}(\mathbb{T}), 36$

limit superior, lim sup, 75

Martin's Axiom, 12

name, 15

canonical, 15

nice, 23

nice name for a function in $\mathbb{B}$, 23

nice name for a function in $\mathbb{P}$, 61

$N_{n}\left({ }^{X} Y\right), 24$

$N_{n}^{\mathbb{P}}\left({ }^{\kappa} \lambda\right), 61$

notion of forcing, 12

adding an unbounded real, 31 collapsing cardinals, 17

preserving cardinals, 17

preserving cofinalities, 17

proper, 32

with Laver property, 34

with Sacks property, 33

$\omega_{\omega}$-bounding, 34

$\mathcal{P}, 70$

partial order

$(\kappa, \lambda)$-distributive, 64

forcing equivalent partial orders, 22 $\kappa$-closed, 30

separative, 9

partial play, 40

partition, 27

refinement of a partition, 27

$\pi(\mathbb{B}), 9$

play, 40

predictor, 70

product of Boolean algebras, 25

$\mathcal{P}^{*}, 70$

$p \downarrow, 8$

r.o. $(\mathbb{P}), 9$

$\mathcal{S}, 33$

separative quotient of a partial order, 10

sequence

cofinal, 6

set

closed unbounded, 6

dense, 8

dense below $p, 8$

empty below $p, 8$

meager, 40

nowhere dense, 40

open dense, 8

stationary, 6

with the Baire property, 41

$\mathcal{S}^{g}, 33$

slalom, 33

strategy, 40

winning strategy, 40

submeasure, 85

Maharam, 85

strictly positive, 85

Suslin algebra, 37

Suslin Hypothesis, SH, 37

$T_{\alpha}, 36$

theorem 
Jensen's Covering Theorem, 8

The Forcing Theorem, 22

The Generic Model Theorem, 15

the Maximum Principle, 20

the Mixing Lemma, 21

tree, 36

ever-branching, 37

$\kappa$-tree, 37

$\lambda$-Suslin, 37

normal, 37

reversed, $\mathbb{T}^{*}, 37$

Suslin, 37

well-pruned, 37

ultrafilter, 11

$\mathbb{B}$-generic, 12

$\lambda$-complete, 11

measurable, 11

normal, 11

$V^{\mathbb{B}}, 13$

$V_{\mathbb{B}}[G], 14$

$V^{\mathbb{P}}, 17$

$V_{\mathbb{P}}[G], 17$

White $(\mathbb{B}), 86$

$0^{\sharp}, 8$ 


\section{Prošireni apstrakt}

\section{Bulove algebre $\mathrm{i}$ forsing}

Bulova algebra $\mathbb{B}$ je kompletna ako svaki neprazan podskup $X \subseteq B$ ima supremum i infimum. Parcijalno uređenje $\mathbb{P}$ je separativno ako za sve $p, q \in$ $P$, ako ne važi $p \leq q$, onda postoji $r \in P$ takvo da $r \leq p$ ali $r \perp q$. Svako separativno parcijalno uređenje $\mathbb{P}$ može se potopiti u kompletnu Bulovu algebru r.o. $(\mathbb{P})$.

Forsing je metod kojim se, polazeći od modela $V$ teorije skupova i koristeći kompletnu Bulovu algebru $\mathbb{B}$ u $V$ i tzv. $\mathbb{B}$-generički ultrafilter $G$ nad $V$, dobija model $V[G]$ takav da: 1) $V \subseteq V[G]$ i $G \in V[G]$; 2) $\operatorname{Ord}^{V[G]}=\operatorname{Ord}^{V}$; 3) ako je $\mathcal{N}$ tranzitivan model teorije $\mathrm{ZF}$ takav da $V \subseteq \mathcal{N}$ i $G \in \mathcal{N}$, onda $V[G] \subseteq \mathcal{N}$; 4) ako $V \models A C$, onda i $V[G] \models A C$. Umesto kompletne Bulove algebre $\mathbb{B}$ može se koristiti i separativno parcijalno uređenje kojem je $\mathbb{B}$ kompletiranje.

Kompletna Bulova algebra $\mathbb{B}$ je $(\kappa, \lambda)$-distributivna ako za svaku kolekciju $\left\langle a_{\alpha \beta}: \alpha<\kappa, \beta<\lambda\right\rangle$ elemenata iz $B$

$$
\bigwedge_{\alpha<\kappa} \bigvee_{\beta<\lambda} a_{\alpha \beta}=\bigvee_{f: \kappa \rightarrow \lambda} \bigwedge_{\alpha<\kappa} a_{\alpha f(\alpha)}
$$

$\mathbb{B}$ je $\kappa$-distributivna ako je $(\kappa, \lambda)$-distributivna za sve beskonačne $\lambda \in$ Card. $\mathbb{B}$ je $(\kappa, \lambda)$-distributivna akko forsing pomoću $\mathbb{B}$ ne dodaje nijednu funkciju $f: \kappa \rightarrow \lambda$.

\section{Igre na Bulovim algebrama}

Prvu beskonačnu igru na Bulovim algebrama, $\mathcal{G}_{\text {inf }}(\omega)$, definisao je Tomas Jeh u radu [15]. Igraju je dva igrača, Beli i Crni, u $\omega$ poteza na Bulovoj algebri $\mathbb{B}$. Na početku Beli bira element $b_{0} \in B^{+}$. Zatim naizmenično Crni bira nenula element $c_{n} \leq b_{n}$, a Beli nenula element $b_{n+1} \leq c_{n}$. Beli igrač pobeđuje akko je $\bigwedge_{n \in \omega} b_{n}=0$. Ova igra može se igrati i na proizvoljnom separativnom parcijalnom uređenju $\mathbb{P}$, i svaki od igrača ima pobedničku strategiju na $\mathbb{P}$ akko je ima na r.o.(P). Jeh je dokazao da Beli igrač ima pobedničku strategiju u $\mathcal{G}_{\text {inf }}(\omega)$ akko $\mathbb{B}$ nije $\omega$-distributivna, i da je dovoljan uslov da bi Crni imao pobedničku strategiju da $\mathbb{P}$ ima $\omega_{1}$-zatvoren gust podskup. $U$ istom radu dat je i primer parcijalnog uređenja (tzv. ,,shooting a club” uređenje) na kojem je igra neodređena: nijedan od igrača nema pobedničku strategiju. 
Prvu cut-and-choose igru, $\mathcal{G}_{\text {dist }}(\omega, 2,2)$, takođe je uveo Jeh u radu [16]. Na početku Beli bira element $p \in B^{+}$. U svakom sledećem potezu Beli bira element $p_{n} \in(0, p)_{\mathbb{B}}\left(\mathrm{tj}\right.$. ,,seče" $p$ na dva dela: $p_{n}^{0}=p_{n}$ i $\left.p_{n}^{1}=p \backslash p_{n}\right)$ a Crni element $i_{n} \in\{0,1\}$ (time ustvari birajući jedan od elemenata $p_{n}^{0}$ i $p_{n}^{1}$ ). Beli pobeđuje akko je $\bigwedge_{n \in \omega} p_{n}^{i_{n}}=0$. Jeh je pokazao da Beli ima pobedničku strategiju akko $\mathbb{B}$ nije $(\omega, 2)$-distributivna.

Sledila su mnoga uopštenja ove igre. Igra $\mathcal{G}_{\text {dist }}(\omega, \lambda, 2)$ igra se na isti način, osim što Beli u svakom potezu seče $p$ na $\lambda$ delova, odnosno bira particiju $A_{n}=\left\{p_{\alpha}^{n}: \alpha<\lambda\right\}$ elementa $p$, a Crni potom bira $\alpha<\lambda$, birajući na taj način jedan od elemenata $p_{\alpha}^{n}$. Ako u ovoj definiciji ne ograničimo veličinu particije $A_{n}$ u $n$-tom potezu Belog, dobijamo igru $\mathcal{G}_{\text {dist }}(\omega, \infty, 2)$ koja je ekvivalentna sa $\mathcal{G}_{\text {inf }}(\omega)$ : svaki od igrača ima pobedničku strategiju u $\mathcal{G}_{\text {dist }}(\omega, \infty, 2)$ akko je ima u $\mathcal{G}_{\text {inf }}(\omega)$.

U drugom uopštenju, igri $\mathcal{G}_{\text {dist }}(\omega, \lambda, \omega)$, Crnom igraču je dozvoljeno da iz particije $A_{n}$ elementa $p$ izabere konačan podskup $B_{n}$. Beli pobeđuje akko je $\bigwedge_{n \in \omega} \bigvee B_{n}=0$. Ispostavlja se da, ako $\mathbb{B}$ nije slabo $(\omega, \lambda)$-distributivna, onda Beli ima pobedničku strategiju u ovoj igri.

Nataša Dobrinen je u radu [9] definisala i igru $\mathcal{G}_{\text {dist }}(\kappa, \lambda, 2)$ koja se igra po istim pravilima kao $\mathcal{G}_{\text {dist }}(\omega, \lambda, 2)$, ali u $\kappa$ poteza. U tom radu dokazano je i da, ako $\mathbb{B}$ nije $(\kappa, \lambda)$-distributivna, onda Beli ima pobedničku strategiju u igri $\mathcal{G}_{\text {dist }}(\kappa, \lambda, 2)$. Obratno, ako Beli ima pobedničku strategiju, onda $\mathbb{B}$ nije ni $\left(\lambda^{<\kappa}, \lambda\right)$-distributivna ni $\left(\kappa, \lambda^{<\kappa}\right)$-distributivna. $\mathrm{U}$ istom radu je konstruisan i primer Bulove algebre na kojoj je igra neodređena.

Igra $\mathcal{G}_{\text {dist }}^{\prime}(\kappa, 2,2)$ slična igri $\mathcal{G}_{\text {dist }}(\kappa, 2,2)$ može se definisati na proizvoljnom parcijalnom uređenju $\mathbb{P}$. Na početku Beli bira element $p \in P$. Zatim u svakom od $\kappa$ poteza Beli bira antilanac $A_{\alpha}$; neka je $A_{\alpha}^{\prime}$ (unapred fiksirani) antilanac takav da su svaka dva elementa $a_{1} \in A_{\alpha}$ i $a_{2} \in A_{\alpha}^{\prime}$ nekompatibilni i $A_{\alpha} \cup A_{\alpha}^{\prime}$ je maksimalan antilanac ispod $p$. Crni potom bira $i_{\alpha} \in\{0,1\}$, birajući na taj način jedan od antilanaca $A_{\alpha}^{0}=A_{\alpha}$ i $A_{\alpha}^{1}=A_{\alpha}^{\prime}$. Beli pobeđuje akko važi $\exists q \leq p \forall \alpha<\kappa\left(A_{\alpha}^{i_{\alpha}} \downarrow\right.$ je gust ispod $\left.q\right)$. Ako se pojam $(\kappa, \lambda)$ distributivnosti na $\mathbb{P}$ definiše tako da je $\mathbb{P}(\kappa, \lambda)$-distributivno akko je r.o.( $\mathbb{P})$ $(\kappa, \lambda)$-distributivno, važi:

Teorema. Beli ima pobedničku strategiju u $\mathcal{G}_{\text {dist }}^{\prime}(\kappa, 2,2)$ na $\mathbb{P}$ akko parcijalno uređenje $\mathbb{P}$ nije $(\kappa, 2)$-distributivno. 
Masaru Kada je u radu [21] definisao još tri igre koje karakterišu Sacks, $\omega^{\omega}$-bounding i Laver parcijalna uređenja.

\section{$\operatorname{Igra} \mathcal{G}_{\mathrm{ls}}(\kappa)$}

Igra $\mathcal{G}_{\text {ls }}(\kappa)$ definisana je $\mathrm{u}$ radu [25] za specijalan slučaj $\kappa=\omega$, a $\mathrm{u}[26] \mathrm{u}$ opštem slučaju. Igra se na kompletnoj Bulovoj algebri $\mathbb{B}$ po istim pravilima kao $\mathcal{G}_{\text {dist }}(\kappa, 2,2)$ : Beli na početku izabere $p \in B^{+}$, a zatim u svakom potezu Beli bira $p_{\alpha} \in(0, p)_{\mathbb{B}}$, a Crni $i_{\alpha} \in\{0,1\}$. Beli pobeđuje akko je $\bigwedge_{\beta<\kappa} \bigvee_{\alpha \geq \beta} p_{\alpha}^{i_{\alpha}}=0$

Teorema. Sledeći uslovi su ekvivalentni:

(a) Beli ima pobedničku strategiju u $\mathcal{G}_{\mathrm{ls}}(\kappa)$ na Bulovoj algebri $\mathbb{B}$;

(b) postoje $p \in B^{+}$i $w:{ }^{<\kappa} 2 \rightarrow(0, p)_{\mathbb{B}}$ takvi da

$$
\forall i: \kappa \rightarrow 2 \bigwedge_{\beta<\kappa} \bigvee_{\alpha \geq \beta} w_{i\lceil\alpha}^{i(\alpha)}=0
$$

(c) postoje $p \in B^{+}$i $w:{ }^{<\kappa} 2 \rightarrow[0, p]_{\mathbb{B}}$ takvi da važi $(*)$;

(d) postoji generička ekstenzija $V_{\mathbb{B}}[G]$ u kojoj postoji podskup $R$ drveta $\left({ }^{<\kappa} 2\right)^{V}$, koji sadrži tačno jedan od elemenata $\varphi^{-} 0$ i $\varphi^{-1}$ za svako $\varphi \in\left({ }^{<\kappa} 2\right)^{V}$ i takav da je za svako $i \in\left({ }^{\kappa} 2\right)^{V} \operatorname{skup}\{\alpha \in \kappa: i\lceil\alpha \in R\}$ ograničen $\mathrm{u} \kappa$.

Tvrđenje analogno ekvivalencijama $(\mathrm{a}) \Leftrightarrow(\mathrm{b}) \Leftrightarrow(\mathrm{c})$ moguće je dokazati i u opštijem obliku, za široku klasu cut-and-choose igara. Kao posledica gornje teoreme dobija se

Teorema. Važi $(\mathrm{a}) \Rightarrow(\mathrm{b}) \Rightarrow(\mathrm{c})$, gde

(a) U nekoj generičkoj ekstenziji $V_{\mathbb{B}}[G], \kappa$ je regularan kardinal a $\left(2^{\kappa}\right)^{V}$ je kolapsirano na $\kappa$;

(b) Beli ima pobedničku strategiju u igri $\mathcal{G}_{\mathrm{ls}}(\kappa)$ na $\mathbb{B}$;

(c) U nekoj generičkoj ekstenziji $V_{\mathbb{B}}[G]$, skupovi $\left({ }^{\kappa} 2\right)^{V}$ i $\left({ }^{<\kappa} 2\right)^{V}$ su iste kardinalnosti.

Za $\kappa=\omega$ dobija se glavni rezultat rada [25]: 
Teorema. Beli ima pobedničku strategiju u $\mathcal{G}_{\mathrm{ls}}(\omega)$ na $\mathbb{B}$ akko je u nekoj generičkoj ekstenziji $V_{\mathbb{B}}[G] \mathfrak{c}^{V}$ kolapsiran na $\omega$.

Što se tiče pobedničke strategije Crnog, glavni rezultati su sledeći:

Teorema. Ako je $\kappa \geq \pi(\mathbb{B})$, onda Crni ima pobedničku strategiju u $\mathcal{G}_{\mathrm{ls}}(\kappa)$ na $\mathbb{B}$.

Teorema. Ako $\mathbb{B}$ sadrži $\lambda$-zatvoren gust podskup $D \subseteq B^{+}$, onda za svaki beskonačan kardinal $\kappa<\lambda$ Crni ima pobedničku strategiju u $\mathcal{G}_{\mathrm{ls}}(\kappa)$.

Na kraju je pokazano da postoji i kompletna Bulova algebra na kojoj je $\mathcal{G}_{\mathrm{ls}}(\kappa)$ neodređena:

Teorema. Za svaki regularan kardinal $\kappa$ za koji važi $\kappa^{<\kappa}=\kappa$ i $\diamond_{\kappa^{+}}(E(\kappa))$ postoji $\kappa^{+}$-Suslinovo drvo $\mathbb{T}$ takvo da je $\mathcal{G}_{\mathrm{ls}}(\kappa)$ neodređena na algebri $\mathbb{B}=$ r.o. $\left(\mathbb{T}^{*}\right)$. 


\section{Biografija}

Šobot Boris je rođen 14. juna 1977. godine u Novom Sadu. Završio je gimnaziju ,,Jovan Jovanović Zmaj" u Novom Sadu. Diplomirao je matematiku na smeru Profesor matematike Prirodno-matematičkog fakulteta u Novom Sadu 2000. godine sa prosečnom ocenom 10.00, a potom i informatiku na smeru Diplomirani informatičar istog fakulteta 2002. godine.

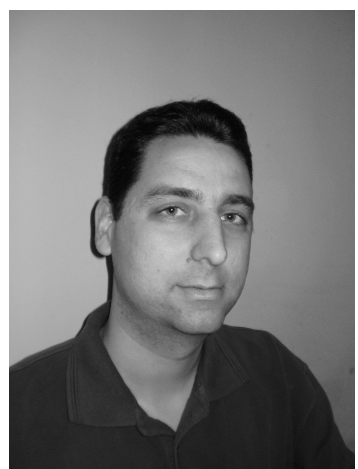

Postdiplomske studije iz matematike, smer Algebra i matematička logika, pohađao je od 2000. do 2004. godine na Prirodno-matematičkom fakultetu u Novom Sadu. Odbranom magistarske teze pod nazivom ,Ultrafiltri i ultraproizvodi" stekao je zvanje magistra matematičkih nauka. Teza je iste godine dobila nagradu ,Mileva Marić-Ajnštajn” kao najbolja iz oblasti matematike i informatike na Univerzitetu u Novom Sadu.

Od 2000. do 2004. godine radio je na Prirodno-matematičkom fakultetu kao asistent-pripravnik, a od 2005. kao asistent. Držao je vežbe iz predmeta: Elementi matematičke logike, Osnovi algebre, Kombinatorika, Teorija grafova, Diskretna matematika, Osnovi geometrije I i II, Formalni jezici i automati i Analiza algoritama. Kao saradnik u gimnaziji „,J. J. Zmaj” drži nastavu iz Računarstva i informatike, kao i dodatnu nastavu iz matematike.

Oblast naučnog interesovanja mu je teorija skupova. Ima objavljena 3 naučna rada, kao i učešća na nekoliko domaćih i međunarodnih konferencija. Član je projekta Forsing, skup-teoretska topologija i teorija modela finansiranog od strane Ministarstva za nauku i tehnološki razvoj Republike Srbije.

Novi Sad, april 2009.

mr Boris Šobot 



\section{UNIVERSITY OF NOVI SAD FACULTY OF SCIENCE KEY WORDS DOCUMENTATION}

Accession number:

ANO

Identification number:

INO

Document type: Monograph type

DT

Type of record: Printed text

TR

Contents code: $\mathrm{PhD}$ dissertation

$\mathrm{CC}$

Author: Boris Šobot, MSc

AU

Mentor: Professor Miloš Kurilić, PhD

$\mathrm{MN}$

Title: Games on Boolean algebras

XI

Language of text: English

LT

Language of abstract: English/Serbian

LA

Country of publication: Serbia

$\mathrm{CP}$

Locality of publication: Vojvodina

LP 
Publication year: 2009

PY

Publisher: Author's reprint

$\mathrm{PU}$

Publication place: Novi Sad, Faculty of Science, Trg Dositeja Obradovića 4 $\mathrm{PP}$

Physical description: 3/114/38/0/0/0

(chapters/pages/citations/pictures/graphs/appendices)

$\mathrm{PD}$

Scientific field: Mathematics

$\mathrm{SF}$

Scientific discipline: Set Theory

$\mathrm{SD}$

Key words: Boolean algebras, partial orders, games, forcing

$\mathrm{UC}$

Holding data: Library of the Department of Mathematics and Informatics, Novi Sad

HD note:

Abstract: The method of forcing is widely used in set theory to obtain various consistency proofs. Complete Boolean algebras play the main role in applications of forcing. Therefore it is useful to define games on Boolean algebras that characterize their properties important for the method. The most investigated game is Jech's distributivity game, such that the first player has the winning strategy iff the algebra is not $(\omega, 2)$-distributive.

We define another game characterizing the collapsing of the continuum to $\omega$, prove several sufficient conditions for the second player to have a winning strategy, and obtain a Boolean algebra on which the game is undetermined. $\mathrm{AB}$

Accepted by the Scientific Board on: ASB 
Defended on:

$\mathrm{DE}$

Thesis defend board:

President: Milan Grulović, PhD, Full Professor at Faculty of Science, University of Novi Sad

Mentor: Miloš Kurilić, PhD, Full Professor at Faculty of Science, University of Novi Sad

Member: Stevan Pilipović, PhD, Full Professor at Faculty of Science, University of Novi Sad

Member: Žarko Mijajlović, PhD, Full Professor at Faculty of Mathematics, University of Belgrade

DB 


\section{UNIVERZITET U NOVOM SADU PRIRODNO-MATEMATIČKI FAKULTET \\ KLJUČNA DOKUMENTACIJASKA INFORMACIJA}

Redni broj:

RBR

Identifikacioni broj:

IBR

Tip dokumentacije: monografska dokumentacija

TD

Tip zapisa: tekstualni štampani materijal

$\mathrm{TZ}$

Vrsta rada: doktorska disertacija

VR

Autor: mr Boris Šobot

AU

Mentor: prof. dr Miloš Kurilić

$\mathrm{MN}$

Naslov rada: Igre na Bulovim algebrama

MR

Jezik publikacije: engleski

JP

Jezik izvoda: engleski/srpski

JI

Zemlja publikovanja: Srbija

$\mathrm{ZP}$

Uže geografsko područje: Vojvodina

UGP 
Godina: 2009

GO

Izdavač: autorski reprint

IZ

Mesto i adresa: Novi Sad, Prirodno-matematički fakultet, Trg Dositeja Obradovića 4

MA

Fizički opis rada: 3/114/38/0/0/0

(poglavlja/ strane/literarnih citata/slika/grafika/priloga)

FO

Naučna oblast: matematika

$\mathrm{NO}$

Naučna disciplina: teorija skupova

ND

Ključne reči: Bulove algebre, parcijalna uređenja, igre, forsing

$\mathrm{PO}$

UDK:

Čuva se: u biblioteci Departmana za matematiku i informatiku, Novi Sad

ČU

Važna napomena:

$\mathrm{VN}$

Izvod: Forsing je metod široko korišćen u teoriji skupova za dokaze konsistentnosti. Kompletne Bulove algebre igraju glavnu ulogu u primenama forsinga. Stoga je korisno definisati igre na Bulovim algebrama koje karakterišu njihove osobine od značaja za taj metod. Najbolje proučena je Jehova igra, koja ima osobinu da prvi igrač ima pobedničku strategiju akko algebra nije $(\omega, 2)$-distributivna.

U tezi definišemo još jednu igru, koja karakteriše kolaps kontinuuma na $\omega$, dokazujemo nekoliko dovoljnih uslova da bi drugi igrač imao pobedničku strategiju, i konstruišemo Bulovu algebru na kojoj je igra neodređena. 
Datum prihvatanja teme od strane NN veća:

DP

Datum odbrane:

DO

Članovi komisije:

Predsednik: dr Milan Grulović, redovni profesor Prirodno matematičkog fakulteta Univerziteta u Novom Sadu

Mentor: dr Miloš Kurilić, redovni profesor Prirodno matematičkog fakulteta Univerziteta u Novom Sadu

Član: dr Stevan Pilipović, redovni profesor Prirodno matematičkog fakulteta Univerziteta u Novom Sadu

Član: dr Žarko Mijajlović, redovni profesor Matematičkog fakulteta Univerziteta u Beogradu

$\mathrm{KO}$ 